\title{
Enhanced perioperative care in liver and pancreat surgery
}

Citation for published version (APA):

Coolsen, M. M. E. (2014). Enhanced perioperative care in liver and pancreat surgery. [Doctoral Thesis, Maastricht University]. Maastricht University. https://doi.org/10.26481/dis.20141031mc

Document status and date:

Published: 01/01/2014

DOI:

10.26481/dis.20141031mc

Document Version:

Publisher's PDF, also known as Version of record

\section{Please check the document version of this publication:}

- A submitted manuscript is the version of the article upon submission and before peer-review. There can be important differences between the submitted version and the official published version of record.

People interested in the research are advised to contact the author for the final version of the publication, or visit the DOI to the publisher's website.

- The final author version and the galley proof are versions of the publication after peer review.

- The final published version features the final layout of the paper including the volume, issue and page numbers.

Link to publication

\footnotetext{
General rights rights.

- You may freely distribute the URL identifying the publication in the public portal. please follow below link for the End User Agreement:

www.umlib.nl/taverne-license

Take down policy

If you believe that this document breaches copyright please contact us at:

repository@maastrichtuniversity.nl

providing details and we will investigate your claim.
}

Copyright and moral rights for the publications made accessible in the public portal are retained by the authors and/or other copyright owners and it is a condition of accessing publications that users recognise and abide by the legal requirements associated with these

- Users may download and print one copy of any publication from the public portal for the purpose of private study or research.

- You may not further distribute the material or use it for any profit-making activity or commercial gain

If the publication is distributed under the terms of Article $25 \mathrm{fa}$ of the Dutch Copyright Act, indicated by the "Taverne" license above, 
Enhanced perioperative care in liver and pancreatic surgery 
๑) Copyright Mariëlle Coolsen, Maastricht 2014

Layout: Tiny Wouters

Cover: Kim Rauwerdink

Printed by: Offpage

ISBN: 978-94-6182-497-4 


\title{
Enhanced perioperative care in liver and pancreatic surgery
}

\begin{abstract}
ACADEMISCH PROEFSCHRIFT
ter verkrijging van de graad van doctor aan de Universiteit Maastricht, op gezag van de Rector Magnificus, Prof dr. L.L.G. Soete volgens het besluit van het College van Decanen, in het openbaar te verdedigen op vrijdag 31 oktober 2014, om 12:00 uur.
\end{abstract}

door

Mariëlle Maria Etiën Coolsen 
Promotor

Prof. dr. C.H.C. Dejong

\section{Co-promotor}

Dr. S.W.M. Olde Damink

\section{Beoordelingscommissie}

Prof. dr. M.F. von Meyenfeldt (voorzitter)

Prof. dr. W.F. Buhre

Prof. dr. K.C. Conlon (University of Dublin, Trinity College)

Dr. I.H.J.T. de Hingh

Prof. dr. J.M.P. Kleijnen 


\section{CONTENTS}

Chapter 1 Introduction and outline of the thesis

Part I Critical appraisal of literature: ERAS programs in HPB surgery and endpoints in pancreatic surgery

Chapter 2 Systematic review and meta-analysis of enhanced recovery after pancreatic surgery with particular emphasis on pancreaticoduodenectomies

Chapter 3 A systematic review of outcomes in patients undergoing liver surgery in an enhanced recovery after surgery pathway

Chapter 4 To drain or not to drain: A cumulative meta-analysis regarding the use of routine abdominal drains after pancreaticoduodenectomy

Chapter 5 Development of a composite endpoint for randomized controlled trials in pancreaticoduodenectomy

Part II Development of ERAS guidelines and a multicenter trial for pancreaticoduodenectomy

Chapter 6 Guidelines for perioperative care for pancreaticoduodenectomy:

Enhanced Recovery After Surgery (ERAS ${ }^{\circ}$ ) Society recommendations

Chapter 7 Study protocol PANDA trial: PAncreaticoduodeNectomy and

Drainage After surgery:

Multicenter randomized controlled trial of prophylactic abdominal drain versus no drain policy after pancreaticoduodenectomy, within an enhanced recovery after surgery pathway

Part III Evaluation of implementing an ERAS program in liver and pancreatic surgery

Chapter 8 Initial experience with a multimodal enhanced recovery program in patients undergoing liver resection

Chapter 9 Improving outcome after pancreaticoduodenectomy- experiences with implementing an enhanced recovery after surgery program

Chapter 10 Implementing an enhanced recovery program after pancreaticoduodenectomy in elderly patients - is it feasible? 
Chapter 11 Attitudes of patients and care providers to enhanced recovery after surgery programs following major abdominal surgery

Chapter 12 General discussion and summary

Short summary

221

Summary in Dutch

225

Valorization

229

Appendices

List of publications

261

Dankwoord

265

Curriculum vitae

271 


\section{CHAPTER 1}

Introduction and outline of the thesis 
Chapter 1 


\subsection{INTRODUCTION}

Liver and pancreatic resections are complex surgical procedures, which can cause considerable surgical stress and possible disruption of metabolic and pulmonary function. Improved operative techniques and insight into perioperative management has lowered mortality after liver resection to its current level of $5 \%$ but morbidity rates remain high, ranging between $30 \%$ and $50 \%{ }^{1-3}$. In large series morbidity rates for pancreatic resections can be as high as 40 to $60 \%{ }^{4-6}$. In order to improve postoperative outcome it is now recommended to centralize these major abdominal procedures in specialized, high-volume clinics. Because of this, mortality rates for pancreaticoduodenectomy (PD), which is the most common form of pancreatic resection, have decreased from 9.8 to $5.1 \%$ in the Netherlands ${ }^{7}$. Postoperative length-of-stay (LOS) after PD varies from 14 to 20 days in different studies ${ }^{8-10}$.

In many hospitals current perioperative care for liver and pancreatic surgery is still conservative. Nasogastric tubes are left in place for days after surgery with the patient either receiving no food at all or only through a needle jejunostomy ${ }^{8}$. Even though this delivers artificial nutrients it is an intervention that bypasses the cephalic-vagal digestive reflex and appears to be associated with considerable risk ${ }^{11,12}$. Other conservative practices are late postoperative mobilization and placement of drains at the end of the surgical procedure ${ }^{5,8,13,14}$. It has been shown that in liver surgery the placement of abdominal drains is not effective and may even increase the risk of intraabdominal infections ${ }^{15-17}$. Similarly, in pancreatic surgery the use of abdominal drains is supported by little evidence. In a randomized controlled trial (RTC) comparing routine drainage versus no drainage after pancreatic surgery, the incidence of intra-abdominal collections of fistulas turned out to be higher after drainage ${ }^{18}$. Fasting from midnight also remains common practice in elective (liver and pancreatic) surgery even though this is not supported by evidence ${ }^{19}$. It has been shown that patients should be allowed to drink clear fluids up to 2 hours and ingest solid food up to 6 hours before surgery ${ }^{20}$.

Enhanced Recovery After Surgery (ERAS) programs, also referred to as fast track, clinical or critical pathway programs, are multimodal, standardized care protocols implemented for a specific surgical field. An ERAS program addresses a variety of evidence-based perioperative interventions and demands a multidisciplinary approach in which surgeons, anesthesiologists, intensive care staff, and nurses work together as a team. The purpose of an ERAS program is to accelerate postoperative recovery and shorten postoperative length of stay (LOS) without increasing or even lowering morbidity and readmission rates. ERAS programs have been successfully implemented in colonic surgery and appear to reduce the length of stay and complication rates without compromising patient safety ${ }^{21,22}$. The LAFA trial compared open colonic resection with laparoscopic resection using either an ERAS protocol or a standard care 
protocol. This trial showed that laparoscopy combined with an ERAS protocol resulted in the optimal perioperative treatment of patients undergoing colonic resections ${ }^{23}$. Numerous other studies have been published on the implementation of ERAS programs in many other surgical fields (e.g. musculoskeletal ${ }^{24}$, breast $^{25}$, aortic ${ }^{26,27}$, bariatric ${ }^{28}$, and prostate surgery ${ }^{29}$ ). All of these studies showed a decrease in LOS without increasing postoperative morbidity or mortality.

Apart from a reduction in postoperative LOS the implementation of an ERAS program may also reduce complication rates after liver and pancreatic surgery. Concerns have been raised, however, regarding the safety of ERAS programs for these upper gastrointestinal (GI) procedures since they are still associated with relatively high morbidity rates and excite a considerable stress response. A nil-by-mouth regimen is still widely practiced because of fear for gastric distention and anastomotic leakage $\mathrm{e}^{30-32}$. Especially in PD, where complications as delayed gastric emptying (DGE) regularly occur, there is a reluctance to allow volitional intake of normal food during the immediate postoperative period $^{33}$. This reluctance, however, is not justified by evidence obtained from randomized trials and may even be considered "obsolete" care $^{34,35}$. Even so, liver and pancreatic procedures are relatively infrequent procedures for which it may be difficult to implement an ERAS program that requires close collaboration between all caregivers. Anesthesiologists in particular must be willing to cooperate and apply all perioperative ERAS components. This includes allowing oral carbohydrate loading up to 2 hours before surgery, epidural anesthesia and the removal of nasogastric tubes directly after surgery.

Given the success of ERAS programs in colonic surgery as well as in many other surgical areas and the abundant evidence for the individual elements of the ERAS protocol we hypothesized that it should be possible to implement an ERAS program for liver and pancreatic surgery as well. Reducing the surgical stress response, thereby enhancing recovery, might even lower procedure-related morbidity. The reluctance to abandon conservative care surrounding upper GI surgery should be overcome thereby allowing room for the practice of evidence-based care. In this thesis we describe how we developed, implemented and evaluated ERAS programs for liver and pancreatic surgery. Most of the studies were carried out at Maastricht University Medical Center (MUMC) in Maastricht, the Netherlands. This is a tertiary referral center for HepatoPancreatico-Biliary (HPB) surgery of relatively high volume (90-100 liver resections and 45-65 pancreatic resections per year).

Our approach consisted of the following steps. First, we critically appraised the literature regarding earlier ERAS programs for liver and pancreatic surgery and prophylactic drainage after pancreatic surgery, which is one of the elements of the ERAS protocol. Second, we performed a study to enable research into PD by proposing uniform definitions and a composite endpoint to be used in further trials. Third, the 
development of perioperative ERAS guidelines for PD was performed in close collaboration with the international ERAS society. Also, a randomized controlled trial was set-up in a multicenter, international setting in order to investigate the use of prophylactic drainage after PD for patients managed in an ERAS protocol. Fourth, the development and implementation of the ERAS program for liver surgery was carried out at MUMC and in the liver unit of the Royal Infirmary in Edinburgh, UK. The implementation and evaluation of the ERAS protocol for PD was carried out at MUMC only. Finally, we performed an international survey among both patients and caregivers in order to evaluate the relative importance of the different elements of an ERAS program.

\subsection{BACKGROUND}

\section{Changes in traditional care}

Until the early nineties perioperative care after abdominal surgery was very conservative. Patients were subjected to a preoperative fasting period lasting up to 29 hours $^{36}$. Large incisions were common and patients often suffered from peroperative hypothermia and intravenous fluids were used abundantly resulting in patients frequently leaving the operating theatre with a grossly positive fluid balance. The postoperative period after abdominal surgery was characterized by prolonged bed rest. Nasogastric tubes and intra-abdominal drains were left in place and patients were kept nil by mouth for days. Furthermore, pain control was not optimal ${ }^{37,38}$.

From the nineties onwards anesthetic and analgesic techniques improved. For example, anesthesiologists started to use rapid short-acting volatile anesthetics (e.g. desflurane and sevoflurane) which shortened the early recovery period in the Post Anesthesia Care Unit (PACU). Furthermore, certain analgesics (remifentanil) ${ }^{39}$ and new analgesic techniques such as neurogenic blockade, local nerve block and epidural analgesia, were introduced $^{40,41}$. Also, surgical techniques improved because of the development of minimally invasive (open) and laparoscopic surgery. These advances in anesthesia and surgery together led to a significant reduction of the early and intermediate recovery phase. Already in the operating theatre patients in ambulatory surgery started to regain vital functions which allowed them to be transferred directly to the day care unit thereby foregoing the need for recovery in a $\mathrm{PACU}^{42}$.

\section{Fast track surgery}

"Fast tracking", a term used to describe a streamlined postoperative treatment pathway in order to shorten recovery, was first applied in ambulatory surgery. Procedures that previously required a multi-day admission, e.g. laparoscopic cholecystectomy ${ }^{43}$, gastro-esophageal reflux surgery ${ }^{44}$ and mastectomy ${ }^{45}$, could now be 
performed in an ambulatory environment with a 1-day stay. Fast tracking not only appeared to accelerate recovery of the patient but also reduced the workload of nursing staff and decreased hospital costs ${ }^{42,46}$. Because of its success the fast track concept was soon applied to more complex surgical procedures such as pneumonectomies ${ }^{47}$, prostatectomies ${ }^{29}$, colectomies $^{48}$, total hip and knee replacements ${ }^{49}$, and various vascular procedures ${ }^{27,50}$. The fast tracking approach allowed these procedures to be performed in a short-stay environment.

\section{Multimodal rehabilitation}

In 1997 Kehlet was the first to describe an extension of the fast track concept which he termed multimodal rehabilitation ${ }^{51}$. Kehlet hypothesized that multimodal interventions in perioperative care could lead to a reduction of the surgical stress response as well as a reduction in organ dysfunction thereby accelerating recovery and reducing postoperative morbidity. An improved understanding of the pathophysiology of the different components of the surgical stress response led to the conclusion that it cannot be modified by any single technique or drug. Rather, a multimodal approach is necessary to positively influence the response, thereby improving surgical outcome. Several factors were identified that contribute to a delayed recovery (Figure 1.1).

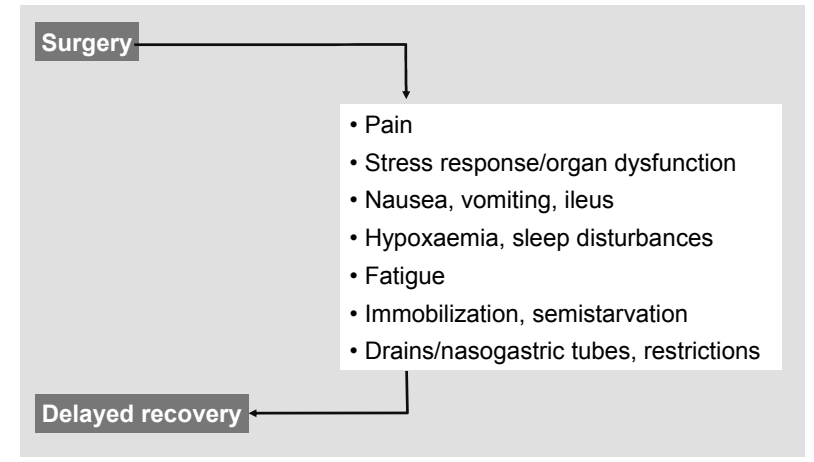

Figure 1.1 Factors contributing to postoperative morbidity.

For instance, prolonged bed rest is known to result in muscle atrophy and weakness, it impairs pulmonary function and predisposes the patient to venous thrombo-embolism $(\mathrm{VTE})^{52}$. Scheduled early mobilization under the guidance of a physiotherapist can prevent these negative effects and may lead to faster recovery. Postoperative pain amplifies the surgical stress response and increases organ dysfunction but could be managed by better analgesic techniques. The aforementioned factors are just a few reasons for a prolonged recovery after surgery. A multimodal rehabilitation program addresses all factors that are known to delay recovery (Figure 1.2) . $^{53}$. 


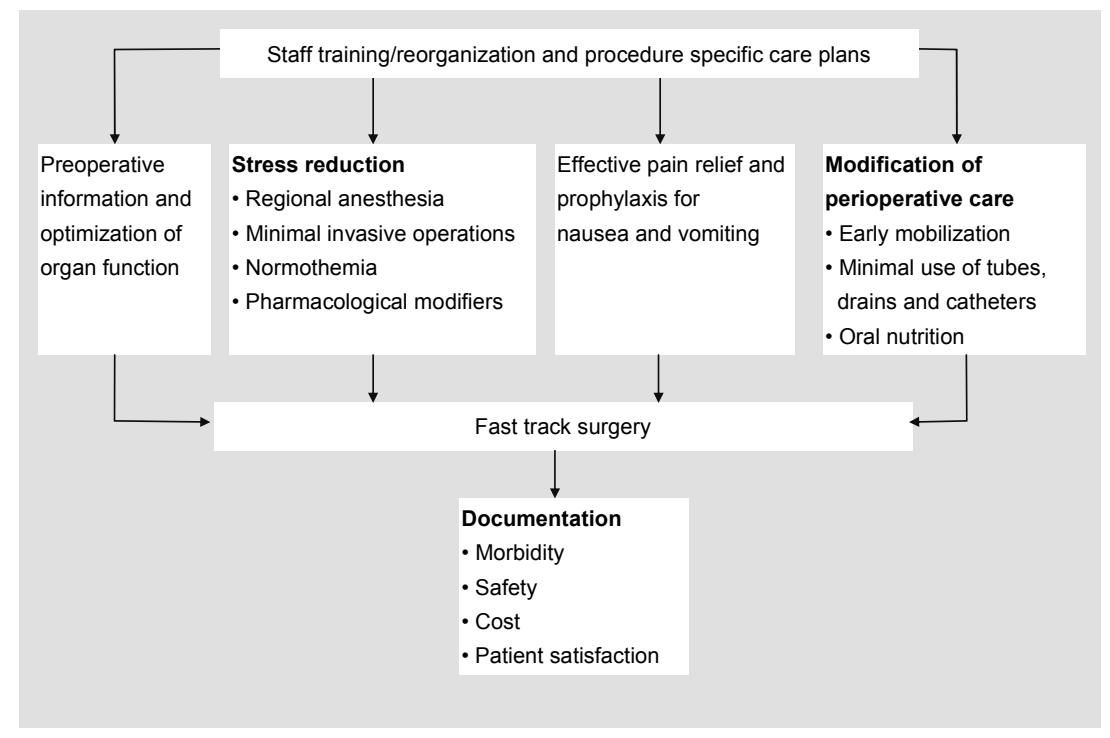

Figure 1.2 Interventions needed for major improvement in surgical outcome.

\section{Enhanced recovery after surgery}

In 2001 the Enhanced Recovery After Surgery (ERAS) study group was established to further develop the concept of multimodal surgical care as proposed by Kehlet et al. This group, consisting of members from five North-European countries (UK, Denmark, Norway, Sweden and The Netherlands) established early on that surgical care all over the world often relies on a wide variety of traditions. Furthermore, the group concluded that a discrepancy exists between optimal practice, as described in scientific literature, and actual practice. This incited the group to examine the process of changing from actual practice, based on traditions, to optimal practice, based on scientific evidence. The group identified several key factors that keep patients in the hospital after surgery. Among these are (1) the need for parenteral analgesia, (2) the need for intravenous fluids secondary to gut dysfunction and (3) bed rest caused by lack of mobility. Subsequently, the group developed ERAS guidelines addressing these factors. In 2005 the group published an evidence-based ERAS consensus protocol for patients undergoing colonic surgery ${ }^{54}$. The most important elements of this protocol are shown in Figure 1.3. 


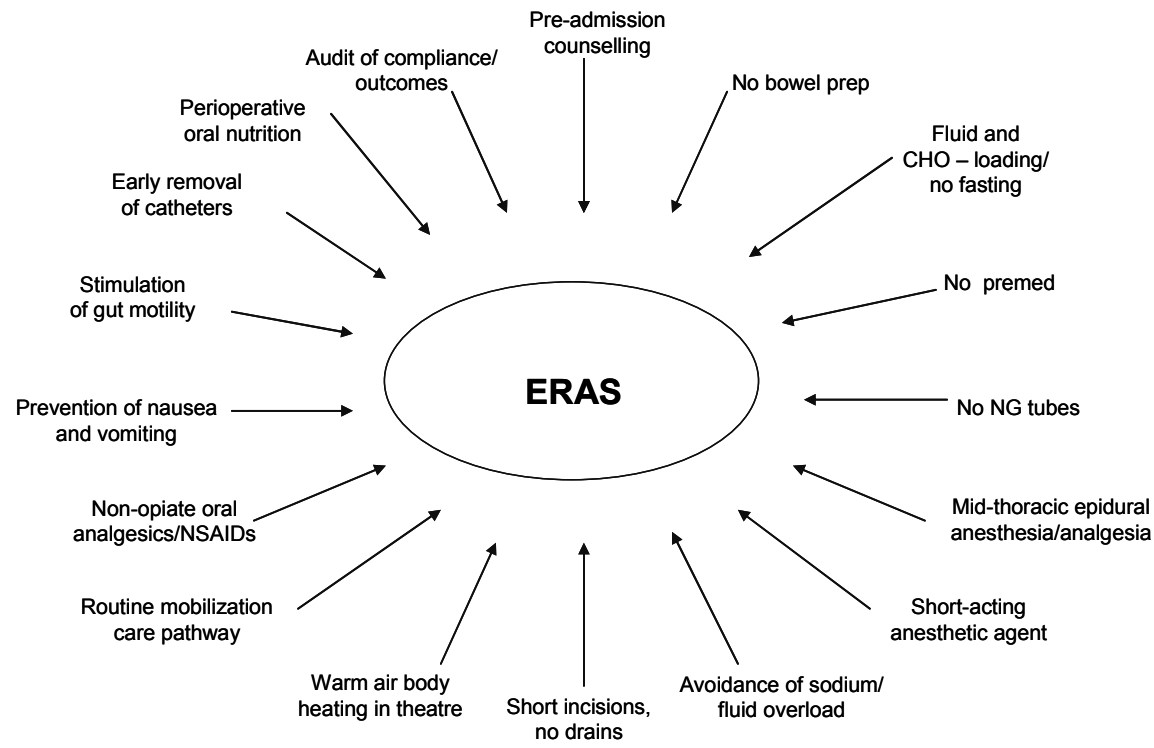

Figure 1.3 Elements of the ERAS protocol for colonic surgery.

Actual practice was evaluated for colonic surgery in a case mix of both conventional and fast track care performed in hospitals of the five participating countries of the ERAS group $^{55} .451$ consecutive patients were included of which 118 in the fast track unit in Denmark and 333 in the other units practicing traditional care. There were no differences in morbidity or 30-day mortality between the different centers. The median length of stay was 2 days in Denmark and $7-9$ days in the other centers $(p<0.05)$. However, readmission rates were higher in the fast track group (22\%) as compared to the traditional care group in the other centers $(2-16 \%, p<0.05)$. After this study into ERAS for colonic surgery, more studies have followed. Meta-analyses of these studies have shown that the use of an ERAS pathway can reduce postoperative complications by up to $50 \%{ }^{21,56}$. Additionally, hospital costs are lower when patients are treated in an ERAS pathway ${ }^{57-60}$.

In 2010 the ERAS society was officially founded and stated its mission as follows: "to develop perioperative care and to improve recovery through research, audit, education and implementation of evidence-based practice." In 2013, the ERAS society published several recommendations and guidelines for ERAS perioperative care in colon ${ }^{61,62}$, rectal/pelvic surgery ${ }^{63,64}$ and pancreaticoduodenectomy (chapter 6) ${ }^{65,66}$. The ERAS society also offers an ERAS training program, which can help perioperative care teams to implement the protocol and maintain high compliance levels to the protocol standard. 


\subsection{THE SURGICAL STRESS RESPONSE AND ERAS}

Surgery provokes a complex stress response, which is associated with endocrinemetabolic changes inducing hypermetabolism and catabolism. Also, surgical injury induces an inflammatory response with activation of a humoral cascade causing hyperthermia and immunosuppression ${ }^{67}$. The aim of an ERAS program is to attenuate the surgical stress response by means of multimodal interventions. A recent randomized controlled trial (RTC) compared 299 patients undergoing colonic surgery based on an ERAS protocol with 298 patients receiving conventional care. In this study outcomes relating to nutritional, metabolic and stress index were measured on postoperative day (POD) 1 and $5^{60}$. The insulin resistance index of the ERAS group appeared to be lower than that of the control group on POD $1(p<0.001)$. Cortisol levels of the control group were elevated on POD 1 and 5, while cortisol levels in the ERAS group remained stable until POD $5(p=0.001)$. Levels of TNF-a, IL-1b, IL-6, and IFN-c were reduced in the ERAS group indicating a lower postoperative stress response. Additionally, gastro-intestinal function recovered faster in the ERAS group as compared to the group receiving conventional care.

\section{Intraoperative hypothermia}

Unintended hypothermia occurs in approximately half of the patients undergoing a surgical procedure. In abdominal operations lasting over 2 hours a drop in core temperature of 2-4 degrees is not unusual. This decline in body temperature stimulates cortisol and catecholamine release which consequently enhances the stress response to surgery ${ }^{68}$. Furthermore, perioperative hypothermia is associated with a significantly increased intraoperative blood loss ${ }^{69,70}$, a higher risk of operative and postoperative cardiac events ${ }^{71}$, a 3 -fold increase in the risk of wound infections ${ }^{72}$, an increased catabolism and increased discomfort ${ }^{73}$. Active prevention of hypothermia by heating the patient greatly reduces these risks, attenuating the stress of operation and improving outcome. For this reason, it is one of the targets of the ERAS program.

\section{Analgesia}

Postoperative pain triggers a surgical stress response and activates the autonomic system, which adversely influences different organ systems. The stress response induced by infusion of neuroendocrine hormones and cytokines causes tachycardia, fever and shock and increases minute ventilation ${ }^{74}$. Epidural analgesia is known to modulate this stress response. Also, it appears that the use of regional anesthetic techniques (like epidural analgesia) using local anesthetics is indeed the best way to reduce adrenocortical, pituitary and sympathetic responses to surgery. Continuous epidural analgesia for a period of up to 24 to 48 hours has the largest inhibitory effect on the abovementioned negative responses to surgery ${ }^{75}$. In abdominal surgery, a meta- 
analysis showed that continuous epidural analgesia, with or without the use of opioids, offers the best pain control as compared to intravenous opioids and allows for early mobilization $^{76}$. Another meta-analysis demonstrated that the use of epidural analgesia results in fewer pulmonary infections and improved pulmonary function ${ }^{77}$. More importantly, epidural analgesia also appears to improve gastrointestinal function and therefore contributes to a faster recovery as compared to opioids ${ }^{78,79}$. For this reason, epidural analgesia currently is the recommended analgesic regimen in ERAS protocols for abdominal surgery.

\section{Postoperative ileus}

Postoperative ileus (POI) is an undesirable event after abdominal surgery which increases patient discomfort and delays nutritional intake and recovery. $\mathrm{POI}$ is one of the major causes of prolonged length of stay (LOS) ${ }^{80}$ and increases in hospital morbidity, regardless of the specific type of surgery ${ }^{81}$. The relationship between POI, which is based on the disturbance of the migration motor complex, and tissue inflammation, as a reaction to surgical stress, is a recent area of interest ${ }^{82}$. Surgical manipulation of the bowels leads to paralysis of the mucosal myocytes and POI as a result. Minimally invasive surgery (as part of the ERAS concept) limits surgical trauma and has been shown to reduce POI significantly ${ }^{83}$. Furthermore, opioids are known to have negative effects on gastrointestinal (GI) motility and may lead to GI paralysis. The use of opioids as postoperative analgesia should therefore be reduced and preferably be replaced by an alternative analgesic medication, e.g. non-steroidal antiinflammatory drugs (NSAIDs) ${ }^{38}$. In colonic surgery, a RCT showed that earlier return of gut function can be achieved with the use of clear carbohydrate-loaded drinks up to 2 hours before surgery ${ }^{84}$. Moreover, preoperative carbohydrate loading decreases postoperative insulin resistance and reduces hunger, thirst and anxiety ${ }^{85-87}$. Routine use of laxatives has been shown to result in a quicker gastrointestinal recovery with earlier bowel movements in a RCT among patients undergoing liver resection ${ }^{88}$. Also, in pancreatic surgery, in a series of 255 pancreatic resections (almost 60\% PDs), oral administration of magnesium and lactulose in addition to metoclopramide on POD 1 supported early start of normal bowel function ${ }^{89}$. So, reduction of opioid use, carbohydrate-loaded drinks up to 2 hours before surgery and use of laxatives are recommended in the ERAS protocol.

\subsection{STANDARDIZATION OF OUTCOMES}

Meta-analyses and RCTs deliver the highest level of evidence about current practice. Conclusions of meta-analyses and large RCTs are incorporated in the latest guidelines and help to optimize care and outcome in surgical practice. In the field of pancreatic 
surgery, however, very few RCTs have been performed ${ }^{90,91}$. An important factor contributing to this is the large number of patients needed to adequately power RCTs for relevant clinical single endpoints. Furthermore, definitions used in different trials into pancreatic surgery are not uniform which complicates the comparability of trials and the conduct of meta-analysis. A composite endpoint (CEP) is a minimal set of outcomes that can be used as a primary endpoint in RCTs. It facilitates the conduct of RCTs by reducing the number of patients needed to adequately power an RCT. The single endpoints included in a composite endpoint are well defined and agreed upon by experts worldwide. Composite endpoints are already widely used in cardiovascular and pulmonary research ${ }^{92-96}$ and have recently been developed for liver surgery by our group ${ }^{97}$. To also improve research and standardization of outcomes in pancreatic surgery we investigated the use of uniform definitions and the development of a composite endpoint for pancreaticoduodenectomy.

\subsection{AIMS OF THIS THESIS}

As previously shown, there is abundant evidence demonstrating ERAS programs are preferred over traditional care in colonic surgery. However, the feasibility and implementation of ERAS programs in major operations such as HPB surgery has not yet been demonstrated and might be a challenge because perioperative care in HPB surgery has been conservative for a long time. In this thesis we present the results of our research into the feasibility of ERAS in HPB surgery. More specifically, our aims were the following:

- To critically appraise literature regarding ERAS programs for HPB surgery and endpoints in pancreatic surgery (chapter $2,3,4,5$ )

- To develop perioperative ERAS guidelines for pancreaticoduodenectomy (chapter 6)

- To initiate a trial evaluating the effect of prophylactic intra-abdominal drainage for patients undergoing pancreaticoduodenectomy in an ERAS program (chapter 7)

- To evaluate the implementation of an ERAS program in HPB surgery (chapter $8,9,10,11)$ 


\section{Outline of this thesis}

Part I: Critical appraisal of literature: ERAS programs in HPB surgery and endpoints in pancreatic surgery

In order to (a) assess what is already known about ERAS programs in HPB surgery and (b) compare existing studies we performed a systematic review and meta-analysis of ERAS programs in pancreatic surgery (chapter 2). Chapter 3 describes a systematic review of ERAS programs in liver surgery. In most abdominal surgical procedures prophylactic intra-abdominal drains did not prove to be beneficial and may even be considered harmful. For this reason, one of the components of an ERAS program in colonic surgery is the omission of prophylactic postoperative intra-abdominal drains. However, whether this component is also a candidate for inclusion in an ERAS program for pancreatic surgery is still unclear since drains may indicate a bleeding or leakage of the pancreatic anastomosis. A systematic review and cumulative meta-analysis on prophylactic drainage after pancreatic surgery was performed to evaluate whether there is conclusive evidence to omit prophylactic intra-abdominal drains in an ERAS program for pancreatic surgery (chapter 4). RCTs for pancreatic surgery are rare and often lack sufficient power. Also, there is a need for uniform definitions and outcomes in studies. In chapter 5 the development of a composite endpoint for pancreaticoduodenectomy is described. This endpoint consists of well-defined components and can be used as a primary endpoint in future RCTs. Its use increases the power of RCTs, enhances the ability to compare outcomes between RCTs and allows for better performance of meta-analysis.

Part II: Development of ERAS guidelines and a multicenter trial for pancreaticoduodenectomy

Chapter 6 covers the development of ERAS perioperative guidelines for pancreaticoduodenectomy by the international ERAS group. While consensus was reached on many items, no consensus was reached regarding omission or inclusion of prophylactic intra-abdominal drainage since the evidence from literature remained inconclusive. For this reason, we decided to design a multicenter RCT comparing prophylactic abdominal drainage with a no-drain policy in patients undergoing pancreaticoduodenectomy (chapter 7). All patients in this study are treated according to the developed ERAS guidelines (chapter 6). The composite endpoint for pancreaticoduodenectomy (chapter 5 ) will be used as one of the outcomes.

Part III: Evaluation of implementing an ERAS program in liver and pancreatic surgery

In this part of the thesis the implementation and evaluation of an ERAS program in liver surgery is described (chapter 8). The implementation of an ERAS program for patients undergoing pancreaticoduodenectomy is evaluated in chapter 9. Chapter 10 
demonstrates that it is also feasible to subject patients of advanced age to an ERAS program after pancreatic surgery. Finally, a multicenter international study was performed to evaluate the relative importance of principal components of an enhanced recovery after surgery program by means of a survey of patients, surgeons, nurses, and anesthesiologists (chapter 11). 


\section{REFERENCES}

1. Belghiti J, Hiramatsu K, Benoist S, Massault P, Sauvanet A, Farges O. Seven hundred forty-seven hepatectomies in the 1990s: an update to evaluate the actual risk of liver resection. J Am Coll Surg 2000;191:38-46.

2. Dimick JB, Wainess RM, Cowan JA, Upchurch GR, Jr., Knol JA, Colletti LM. National trends in the use and outcomes of hepatic resection. J Am Coll Surg 2004;199:31-38.

3. Jarnagin WR, Gonen M, Fong Y, DeMatteo RP, Ben-Porat L, Little S, Corvera C, Weber S, Blumgart LH. Improvement in perioperative outcome after hepatic resection: analysis of 1,803 consecutive cases over the past decade. Ann Surg 2002;236:397-406.

4. DeOliveira ML, Winter JM, Schafer M, Cunningham SC, Cameron JL, Yeo CJ, Clavien PA. Assessment of complications after pancreatic surgery: A novel grading system applied to 633 patients undergoing pancreaticoduodenectomy. Ann Surg 2006;244:931-937.

5. Winter JM, Cameron JL, Campbell KA, Arnold MA, Chang DC, Coleman J, Hodgin MB, Sauter PK, Hruban RH, Riall TS, Schulick RD, Choti MA, Lillemoe KD, Yeo CJ. 1423 pancreaticoduodenectomies for pancreatic cancer: A single-institution experience. J Gastrointest Surg 2006;10:1199-1210.

6. Balcom JHt, Rattner DW, Warshaw AL, Chang Y, Fernandez-del Castillo C. Ten-year experience with 733 pancreatic resections: changing indications, older patients, and decreasing length of hospitalization. Arch Surg 2001;136:391-398.

7. de Wilde RF, Besselink MG, van der Tweel I, de Hingh IH, van Eijck CH, Dejong CH, Porte RJ, Gouma DJ, Busch OR, Molenaar IQ; Dutch Pancreatic Cancer Group. Impact of nationwide centralization of pancreaticoduodenectomy on hospital mortality. Br J Surg 2012;99:404-410.

8. Gouma DJ, van Geenen RC, van Gulik TM, de Haan RJ, de Wit LT, Busch OR, Obertop H. Rates of complications and death after pancreaticoduodenectomy: risk factors and the impact of hospital volume. Ann Surg 2000;232:786-795.

9. Richter A, Niedergethmann M, Sturm JW, Lorenz D, Post S, Trede M. Long-term results of partial pancreaticoduodenectomy for ductal adenocarcinoma of the pancreatic head: 25-year experience. World J Surg 2003;27:324-329.

10. Yeo CJ, Cameron JL, Sohn TA, Lillemoe KD, Pitt HA, Talamini MA, Hruban RH, Ord SE, Sauter PK, Coleman J, Zahurak ML, Grochow LB, Abrams RA. Six hundred fifty consecutive pancreaticoduodenectomies in the 1990s: pathology, complications, and outcomes. Ann Surg 1997;226:248-257.

11. Han-Geurts IJ, Verhoef C, Tilanus HW. Relaparotomy following complications of feeding jejunostomy in esophageal surgery. Dig Surg 2004;21:192-196.

12. Lobo DN, Williams RN, Welch NT, Aloysius MM, Nunes QM, Padmanabhan J, Crowe JR, Iftikhar SY, Parsons SL, Neal KR, Allison SP, Rowlands BJ. Early postoperative jejunostomy feeding with an immune modulating diet in patients undergoing resectional surgery for upper gastrointestinal cancer: a prospective, randomized, controlled, double-blind study. Clin Nutr 2006;25:716-726.

13. Bona S, Gavelli A, Huguet C. The role of abdominal drainage after major hepatic resection. Am J Surg 1994;167:593-595.

14. Fuster J, Llovet JM, Garcia-Valdecasas JC, Grande L, Fondevila C, Vilana R, Palacin J, Tabet J, Ferrer J, Bruix J, Visa J. Abdominal drainage after liver resection for hepatocellular carcinoma in cirrhotic patients: a randomized controlled study. Hepatogastroenterology 2004;51:536-540.

15. Gurusamy KS, Samraj K, Davidson BR. Routine abdominal drainage for uncomplicated liver resection. Cochrane Database Syst Rev 2007;18:3;CD006232

16. Uetsuji S, Kwon AH, Komada H, Okuda Y, Imamura A, Kamiyama Y. Clinical evaluation of closed suction drainage following hepatectomy. Surg Today 1997;27:298-301.

17. Petrowsky H, Demartines N, Rousson V, Clavien PA. Evidence-based value of prophylactic drainage in gastrointestinal surgery: a systematic review and meta-analyses. Ann Surg 2004;240:1074-1084.

18. Conlon KC, Labow D, Leung D, Smith A, Jarnagin W, Coit DG, Merchant N, Brennan MF. Prospective randomized clinical trial of the value of intraperitoneal drainage after pancreatic resection. Ann Surg 2001;234:487-493. 
19. Smith I, Kranke P, Murat I, Smith A, O'Sullivan G, Søreide E, Spies C, in't Veld B; European Society of Anaesthesiology. Perioperative fasting in adults and children: guidelines from the European Society of Anaesthesiology. Eur J Anaesthesiol 2011;28:556-569.

20. American Society of Anesthesiologists C. Practice guidelines for preoperative fasting and the use of pharmacologic agents to reduce the risk of pulmonary aspiration: application to healthy patients undergoing elective procedures: an updated report by the American Society of Anesthesiologists Committee on Standards and Practice Parameters. Anesthesiology 2011;114:495-511.

21. Varadhan KK, Neal KR, Dejong CH, Fearon KC, Ljungqvist O, Lobo DN. The enhanced recovery after surgery (ERAS) pathway for patients undergoing major elective open colorectal surgery: a meta-analysis of randomized controlled trials. Clin Nutr 2010;29:434-440.

22. Gillissen F, Hoff C, Maessen JM, Winkens B, Teeuwen JH, von Meyenfeldt MF, Dejong CH. Structured synchronous implementation of an enhanced recovery program in elective colonic surgery in 33 hospitals in The Netherlands. World J Surg 2013;37:1082-1093.

23. Vlug MS, Wind J, Hollmann MW, Ubbink DT, Cense HA, Engel AF, Gerhards MF, van Wagensveld BA, van der Zaag ES, van Geloven AA, Sprangers MA, Cuesta MA, Bemelman WA; LAFA study group. Laparoscopy in combination with fast track multimodal management is the best perioperative strategy in patients undergoing colonic surgery: a randomized clinical trial (LAFA-study). Ann Surg 2011;254: 868-875.

24. Barbieri A, Vanhaecht K, Van Herck P, Sermeus W, Faggiano F, Marchisio S, Panella M. Effects of clinical pathways in the joint replacement: a meta-analysis. BMC Med 2009;7:32.

25. Arsalani-Zadeh R, ElFadl D, Yassin N, MacFie J. Evidence-based review of enhancing postoperative recovery after breast surgery. Br J Surg 2011;98:181-196.

26. Brustia P, Renghi A, Gramaglia L, Porta C, Cassatella R, De Angelis R, Tiboldo F. Mininvasive abdominal aortic surgery. Early recovery and reduced hospitalization after multidisciplinary approach. J CardiovasC Surg (Torino) 2003;44:629-635.

27. Podore PC, Throop EB. Infrarenal aortic surgery with a 3-day hospital stay: A report on success with a clinical pathway. J Vasc Surg 1999;29:787-792.

28. McCarty TM, Arnold DT, Lamont JP, Fisher TL, Kuhn JA. Optimizing outcomes in bariatric surgery: outpatient laparoscopic gastric bypass. Ann Surg 2005;242:494-498.

29. Kirsh EJ, Worwag EM, Sinner M, Chodak GW. Using outcome data and patient satisfaction surveys to develop policies regarding minimum length of hospitalization after radical prostatectomy. Urology 2000;56:101-106.

30. Lassen K, Dejong CH, Ljungqvist O, Fearon K, Andersen J, Hannemann P, von Meyenfeldt MF, Hausel J, Nygren J, Revhaug A. Nutritional support and oral intake after gastric resection in five northern European countries. Dig Surg 2005;22:346-352.

31. Hirao M, Tsujinaka T, Takeno A, Fujitani K, Kurata M. Patient-controlled dietary schedule improves clinical outcome after gastrectomy for gastric cancer. World J Surg 2005;29:853-857.

32. Atkins BZ, Shah AS, Hutcheson KA, Mangum JH, Pappas TN, Harpole DH Jr, D'Amico TA. Reducing hospital morbidity and mortality following esophagectomy. Ann Thorac Surg 2004;78:1170-1176.

33. Tanaka M. Gastroparesis after a pylorus-preserving pancreatoduodenectomy. Surg Today 2005;35: 345-350.

34. Lassen K, Kjaeve J, Fetveit T, Tran $\varnothing$ G, Sigurdsson HK, Horn A, Revhaug A. Allowing normal food at will after major upper gastrointestinal surgery does not increase morbidity: a randomized multicenter trial. Ann Surg 2008;247:721-729.

35. Lewis SJ, Andersen HK, Thomas S. Early enteral nutrition within $24 \mathrm{~h}$ of intestinal surgery versus later commencement of feeding: a systematic review and meta-analysis. J Gastrointest Surg 2009;13: 569-575.

36. Chapman A. (1996) Current theory and practice: a study of pre-operative fasting. Nurs Stand 10:33-36.

37. Kehlet H. Postoperative pain relief--what is the issue? Br J Anaesth 1994;72:375-378.

38. Kehlet H, Rung GW, Callesen T. (1996) Postoperative opioid analgesia: time for a reconsideration? J Clin Anesth 8:441-445.

39. Zarate E, Latham P, White PF, Bossard R, Morse L, Douning LK, Shi C, Chi L. Fast-track cardiac anesthesia: use of remifentanil combined with intrathecal morphine as an alternative to sufentanil during desflurane anesthesia. Anesth Analg 2000;91:283-287. 
40. White PF. Ambulatory anesthesia advances into the new millennium. Anesth Analg 2000;90:1234-1235.

41. White PF, Craig WF, Vakharia AS, Ghoname E, Ahmed HE, Hamza MA. Percutaneous neuromodulation therapy: does the location of electrical stimulation effect the acute analgesic response? Anesth Analg 2000;91:949-954.

42. Duncan PG, Shandro J, Bachand R, Ainsworth L. A pilot study of recovery room bypass ("fast-track protocol") in a community hospital. Can J Anaesth 2001;48:630-636.

43. Smith R, Kolyn D, Pace R. Outpatient laparoscopic cholecystectomy. HPB Surg 1994;7:261-264.

44. Trondsen E, Mjaland O, Raeder J, Buanes T. Day-case laparoscopic fundoplication for gastrooesophageal reflux disease. Br J Surg 2000;87:1708-1711.

45. Tan LR, Guenther JM. Outpatient definitive breast cancer surgery. Am Surg 1997;63:865-867.

46. Watkins AC, White PF. Fast-tracking after ambulatory surgery. J Perianesth Nurs 2001;16:379-387.

47. Tovar EA, Roethe RA, Weissig MD, Lloyd RE, Patel GR. One-day admission for lung lobectomy: an incidental result of a clinical pathway. Ann Thorac Surg 1998;65:803-806.

48. Basse L, Hjort Jakobsen D, Billesbolle P, Werner M, Kehlet $\mathrm{H}$. A clinical pathway to accelerate recovery after colonic resection. Ann Surg 2000;232:51-57.

49. Husted H, Holm G. Fast track in total hip and knee arthroplasty--experiences from Hvidovre University Hospital, Denmark. Injury 2000;37 Suppl 5:S31-35.

50. Collier PE. Are one-day admissions for carotid endarterectomy feasible? Am J Surg 1995;170:140-143.

51. Kehlet $\mathrm{H}$. Multimodal approach to control postoperative pathophysiology and rehabilitation. $\mathrm{Br} J$ Anaesth 1997;78:606-617.

52. Spyropoulos AC, Brotman DJ, Amin AN, Deitelzweig SB, Jaffer AK, McKean SC. Prevention of venous thromboembolism in the cancer surgery patient. Cleve Clin J Med 2008;75 Suppl 3:S17-26.

53. Wilmore DW, Kehlet $\mathrm{H}$. Management of patients in fast track surgery. BMJ 2001;322:473-476.

54. Fearon KC, Ljungqvist O, Von Meyenfeldt M, Revhaug A, Dejong CH, Lassen K, Nygren J, Hausel J, Soop $\mathrm{M}$, Andersen J, Kehlet $\mathrm{H}$. Enhanced recovery after surgery: a consensus review of clinical care for patients undergoing colonic resection. Clin Nutr 2005;24:466-477.

55. Nygren J, Hausel J, Kehlet H, Revhaug A, Lassen K, Dejong C, Andersen J, von Meyenfeldt M, Ljungqvist $\mathrm{O}$, Fearon KC. A comparison in five European Centres of case mix, clinical management and outcomes following either conventional or fast-track perioperative care in colorectal surgery. Clin Nutr 2005;24:455-461.

56. Adamina M, Kehlet H, Tomlinson GA, Senagore AJ, Delaney CP. Enhanced recovery pathways optimize health outcomes and resource utilization: a meta-analysis of randomized controlled trials in colorectal surgery. Surgery 2011;149:830-840.

57. Stephen AE, Berger DL. Shortened length of stay and hospital cost reduction with implementation of an accelerated clinical care pathway after elective colon resection. Surgery 2003;133:277-282.

58. Kariv Y, Delaney CP, Senagore AJ, Manilich EA, Hammel JP, Church JM, Ravas J, Fazio VW. Clinical outcomes and cost analysis of a "fast track" postoperative care pathway for ileal pouch-anal anastomosis: a case control study. Dis Colon Rectum 2007;50:137-146.

59. King PM, Blazeby JM, Ewings P, Franks PJ, Longman RJ, Kendrick AH, Kipling RM, Kennedy RH. Randomized clinical trial comparing laparoscopic and open surgery for colorectal cancer within an enhanced recovery programme. Br J Surg 2006;93:300-308.

60. Ren L, Zhu D, Wei Y, Pan X, Liang L, Xu J, Zhong Y, Xue Z, Jin L, Zhan S, Niu W, Qin X, Wu Z, Wu Z. Enhanced Recovery After Surgery (ERAS) program attenuates stress and accelerates recovery in patients after radical resection for colorectal cancer: a prospective randomized controlled trial. World $\mathrm{J}$ Surg 2012;36:407-414.

61. Gustafsson UO, Scott MJ, Schwenk W, Demartines N, Roulin D, Francis N, McNaught CE, Macfie J, Liberman AS, Soop M, Hill A, Kennedy RH, Lobo DN, Fearon K, Ljungqvist O; Enhanced Recovery After Surgery (ERAS) Society, for Perioperative Care; European Society for Clinical Nutrition and Metabolism (ESPEN); International Association for Surgical Metabolism and Nutrition (IASMEN). Guidelines for perioperative care in elective colonic surgery: Enhanced Recovery After Surgery (ERAS((R))) Society recommendations. World J Surg 2013;37:259-284. 
62. Gustafsson UO, Scott MJ, Schwenk W, Demartines N, Roulin D, Francis N, McNaught CE, MacFie J, Liberman AS, Soop M, Hill A, Kennedy RH, Lobo DN, Fearon K, Ljungqvist O; Enhanced Recovery After Surgery Society. Guidelines for perioperative care in elective colonic surgery: Enhanced Recovery After Surgery (ERAS(R)) Society recommendations. Clin Nutr 2012;31:783-800.

63. Nygren J, Thacker J, Carli F, Fearon KC, Norderval S, Lobo DN, Ljungqvist O, Soop M, Ramirez J; Enhanced Recovery After Surgery Society. Guidelines for perioperative care in elective rectal/pelvic surgery: Enhanced Recovery After Surgery (ERAS(R)) Society recommendations. Clin Nutr 2012;31:801816.

64. Nygren J, Thacker J, Carli F, Fearon KC, Norderval S, Lobo DN, Ljungqvist O, Soop M, Ramirez J; Enhanced Recovery After Surgery (ERAS) Society, for Perioperative Care; European Society for Clinical Nutrition and Metabolism (ESPEN); International Association for Surgical Metabolism and Nutrition (IASMEN). Guidelines for perioperative care in elective rectal/pelvic surgery: Enhanced Recovery After Surgery (ERAS((R))) Society recommendations. World J Surg 2013;37:285-305.

65. Lassen K, Coolsen MM, Slim K, Carli F, de Aguilar-Nascimento JE, Schäfer M, Parks RW, Fearon KC, Lobo DN, Demartines N, Braga M, Ljungqvist O, Dejong CH; Enhanced Recovery After Surgery (ERAS) Society, for Perioperative Care; European Society for Clinical Nutrition and Metabolism (ESPEN); International Association for Surgical Metabolism and Nutrition (IASMEN). Guidelines for perioperative care for pancreaticoduodenectomy: Enhanced Recovery After Surgery (ERAS(R)) Society recommendations. World J Surg 2013;37:240-258.

66. Lassen K, Coolsen MM, Slim K, Carli F, de Aguilar-Nascimento JE, Schäfer M, Parks RW, Fearon KC, Lobo DN, Demartines N, Braga M, Ljungqvist O, Dejong CH; ERAS ${ }^{\circledR}$ Society; European Society for Clinical Nutrition and Metabolism; International Association for Surgical Metabolism and Nutrition. Guidelines for perioperative care for pancreaticoduodenectomy: Enhanced Recovery After Surgery (ERAS(R)) Society recommendations. Clin Nutr 2013;31:817-830.

67. Wilmore DW. From Cuthbertson to fast-track surgery: 70 years of progress in reducing stress in surgical patients. Ann Surg 2002;236:643-648.

68. Frank SM, Higgins MS, Breslow MJ, Fleisher LA, Gorman RB, Sitzmann JV, Raff H, Beattie C. The catecholamine, cortisol, and hemodynamic responses to mild perioperative hypothermia. A randomized clinical trial. Anesthesiology 1995;82:83-93.

69. Winkler M, Akça O, Birkenberg B, Hetz H, Scheck T, Arkiliç CF, Kabon B, Marker E, Grübl A, Czepan R, Greher M, Goll V, Gottsauner-Wolf F, Kurz A, Sessler DI. Aggressive warming reduces blood loss during hip arthroplasty. Anesth Analg 2000;91:978-984.

70. Rajagopalan S, Mascha E, Na J, Sessler DI. The effects of mild perioperative hypothermia on blood loss and transfusion requirement. Anesthesiology 2008;108:71-77.

71. Frank SM, Fleisher LA, Breslow MJ, Higgins MS, Olson KF, Kelly S, Beattie C. Perioperative maintenance of normothermia reduces the incidence of morbid cardiac events. A randomized clinical trial. JAMA 1997;277:1127-1134.

72. Kurz A, Sessler DI, Lenhardt R. Perioperative normothermia to reduce the incidence of surgical-wound infection and shorten hospitalization. Study of Wound Infection and Temperature Group. N Engl J Med 1996;334:1209-1215.

73. Sessler DI. Mild perioperative hypothermia. N Engl J Med 1997;336:1730-1737.

74. Liu S, Carpenter RL, Neal JM. Epidural anesthesia and analgesia. Their role in postoperative outcome. Anesthesiology 1995;82:1474-1506.

75. Kehlet H, Wilmore DW. Multimodal strategies to improve surgical outcome. Am J Surg 2002;183:630641.

76. Block BM, Liu SS, Rowlingson AJ, Cowan AR, Cowan JA, Jr., Wu CL. Efficacy of postoperative epidural analgesia: a meta-analysis. JAMA 2003;290:2455-2463.

77. Popping DM, Elia N, Marret E, Remy C, Tramer MR. Protective effects of epidural analgesia on pulmonary complications after abdominal and thoracic surgery: a meta-analysis. Arch Surg 2008,143:990-999.

78. Jorgensen H, Wetterslev J, Moiniche S, Dahl JB. Epidural local anaesthetics versus opioid-based analgesic regimens on postoperative gastrointestinal paralysis, PONV and pain after abdominal surgery. Cochrane Database Syst Rev 2000;4:CD001893. 
79. Holte K, Kehlet H. Postoperative ileus: progress towards effective management. Drugs 2002;62:26032615.

80. Iyer S, Saunders WB, Stemkowski S. Economic burden of postoperative ileus associated with colectomy in the United States. J Manag Care Pharm 2009;15:485-494.

81. Luckey A, Livingston E, Tache Y. Mechanisms and treatment of postoperative ileus. Arch Surg 2003;138:206-214.

82. Hoffmann $\mathrm{H}$, Kettelhack $\mathrm{C}$. Fast-track surgery--conditions and challenges in postsurgical treatment: a review of elements of translational research in enhanced recovery after surgery. Eur Surg Res 2012;49:24-34.

83. Augestad KM, Delaney CP. Postoperative ileus: impact of pharmacological treatment, laparoscopic surgery and enhanced recovery pathways. World J Gastroenterol 2010;16:2067-2074.

84. Noblett SE, Watson DS, Huong H, Davison B, Hainsworth PJ, Horgan AF. Pre-operative oral carbohydrate loading in colorectal surgery: a randomized controlled trial. Colorectal Dis 2006;8:563-569.

85. Ljungqvist $\mathrm{O}$, Nygren J, Thorell A. Modulation of post-operative insulin resistance by pre-operative carbohydrate loading. Proc Nutr Soc 2002;61:329-336.

86. Hausel J, Nygren J, Lagerkranser M, Hellström PM, Hammarqvist F, Almström C, Lindh A, Thorell A, Ljungqvist $\mathrm{O}$. A carbohydrate-rich drink reduces preoperative discomfort in elective surgery patients. Anesth Analg 2001;93:1344-1350.

87. Helminen $\mathrm{H}$, Viitanen $\mathrm{H}$, Sajanti J. Effect of preoperative intravenous carbohydrate loading on preoperative discomfort in elective surgery patients. Eur J Anaesthesiol 2009;26:123-127.

88. Hendry PO, van Dam RM, Bukkems SF, McKeown DW, Parks RW, Preston T, Dejong CH, Garden OJ, Fearon KC; Enhanced Recovery After Surgery (ERAS) Group. Randomized clinical trial of laxatives and oral nutritional supplements within an enhanced recovery after surgery protocol following liver resection. Br J Surg 2010;97:1198-1206.

89. Berberat PO, Ingold H, Gulbinas A, Kleeff J, Müller MW, Gutt C, Weigand M, Friess H, Büchler MW. Fast track--different implications in pancreatic surgery. J Gastrointest Surg 2007;11:880-887.

90. Kaido T. Recent randomized controlled trials in pancreaticoduodenectomy. Pancreas 2006;33:228-232.

91. Kaido T. Analysis of randomized controlled trials on hepatopancreatic surgery. Dig Dis Sci 2006;51: 1761-1766.

92. Yusuf S, Sleight P, Pogue J, Bosch J, Davies R, Dagenais G. Effects of an angiotensin-converting-enzyme inhibitor, ramipril, on cardiovascular events in high-risk patients. The Heart Outcomes Prevention Evaluation Study Investigators. N Engl J Med 2000;342:145-153.

93. Sinha IP, Gallagher R, Williamson PR, Smyth RL. Development of a core outcome set for clinical trials in childhood asthma: a survey of clinicians, parents, and young people. Trials 2012;13:103.

94. Taylor DW, Barnett HJ, Haynes RB, Ferguson GG, Sackett DL, Thorpe KE, Simard D, Silver FL, Hachinski V, Clagett GP, Barnes R, Spence JD. Low-dose and high-dose acetylsalicylic acid for patients undergoing carotid endarterectomy: a randomised controlled trial. ASA and Carotid Endarterectomy (ACE) Trial Collaborators. Lancet 1999;353:2179-2184.

95. Niewoehner DE, Erbland ML, Deupree RH, Collins D, Gross NJ, Light RW, Anderson P, Morgan NA. Effect of systemic glucocorticoids on exacerbations of chronic obstructive pulmonary disease. Department of Veterans Affairs Cooperative Study Group. N Engl J Med 1999;340:1941-1947.

96. Investigators T. Trial of invasive versus medical therapy in elderly patients with chronic symptomatic coronary-artery disease (TIME): a randomised trial. Lancet 2001;358:951-957.

97. van den Broek MA, van Dam RM, van Breukelen GJ, Bemelmans MH, Oussoultzoglou E, Pessaux P, Dejong $\mathrm{CH}$, Freemantle N, Olde Damink SW. Development of a composite endpoint for randomized controlled trials in liver surgery. Br J Surg 2011;98:1138-1145 
Critical appraisal of literature: ERAS programs in HPB surgery and endpoints in pancreatic surgery 


\section{CHAPTER}

Systematic review and meta-analysis of enhanced

recovery after pancreatic surgery with particular emphasis on pancreaticoduodenectomies

MME Coolsen, RM van Dam, AA van der Wilt, K Slim, K Lassen, CHC Dejong

World J Surg. 2013;37:1909-18 


\section{ABSTRACT}

\section{Background}

In the past decade, Enhanced Recovery after Surgery (ERAS) protocols have been implemented in several fields of surgery. With these protocols, a faster recovery and shorter hospital stay can be accomplished without an increase in morbidity or mortality. The purpose of this study was to review systematically the evidence for implementation of an ERAS protocol in pancreatic resections, with particular emphasis on pancreaticoduodenectomies (PDs).

\section{Methods}

A systematic search was performed in Medline, Embase, Pubmed, CINAHL, and the Cochrane library for papers describing an ERAS program in adult patients undergoing elective pancreatic surgery published between January 1966 and December 2012. The primary outcome measure was postoperative length of stay. Secondary out- come measures were time to recovery of normal function, overall postoperative complication rates, readmissions, and mortality. Subsequently, a meta-analysis of outcome measures focusing on PD was conducted. This systematic review and meta-analysis was performed according to the PRISMA statement.

\section{Results}

The literature search produced 248 potentially relevant papers. Of these, eight papers met the predefined inclusion criteria: five comparative studies with historical controls, two retrospective studies, and one prospective study, describing a total of 1,558 patients. Only three of the studies reported data on discharge criteria and assessed time to recovery and return to normal function. Implementation of an ERAS protocol led in four of five comparative studies to a significant decrease in length of stay (reduction of 2-6 days in different studies). Meta-analysis of four studies focusing on PDs showed that there was a significant difference in complication rates in favor of the ERAS group (absolute risk difference $8.2 \%$, 95\% confidence interval (CI) 2.0-14.4, $\mathrm{p}=0.008$ ). Introduction of an ERAS protocol did not result in an increase in mortality or readmissions. Delayed gastric emptying and incidence of pancreatic fistula did not differ significantly between groups. All studies reporting on hospital costs showed a decrease after implementation of ERAS.

\section{Conclusions}

This systematic review suggests that using an ERAS protocol in pancreatic resections may help to shorten hospital length of stay without compromising morbidity and mortality. This seemed to apply to distal pancreatectomy, total pancreatectomy, and PD. Meta- analysis was performed for those studies focusing on PD and showed that there were no differences in readmission or mortality. Morbidity rates were significantly lower for patients managed according ERAS principles. 


\section{INTRODUCTION}

Recent research in surgery focuses on reducing perioperative surgical stress to decrease the "postoperative dip" and to make patients recover better. Postoperative length of stay is consequently shortened and this reduces costs ${ }^{1}$. In the context of these developments Enhanced Recovery after Surgery (ERAS) programs were introduced. ERAS programs, also referred to as "fast track", "clinical", or "critical pathways", are multimodal, standardized care protocols implemented in a specific field of surgery. An ERAS program addresses a variety of evidence-based perioperative interventions and demands a multidisciplinary approach in which surgeons, anesthesiologists, intensive care staff, and nurses work closely together. The purpose of an ERAS program is to accelerate postoperative recovery and shorten postoperative length of stay (LOS) without an increase in morbidity or readmissions.

Kehlet and coworkers were the first to describe and implement such a multimodal care protocol successfully in colonic surgery ${ }^{2}$. Subsequently, numerous studies have been published on this topic, not only in colonic surgery but also in many other fields of surgery (e.g. musculoskeletal ${ }^{3}$, breast $^{4}$, aortic ${ }^{5,6}$, bariatric ${ }^{7,8}$, and prostate surgery ${ }^{9}$ ). All of these studies showed a decrease in LOS, without increasing postoperative morbidity, or mortality. Pancreatic surgery traditionally has been considered to be high-risk abdominal surgery associated with high morbidity and mortality rates. Advances in diagnostic and surgical techniques in the past decades and improved management of intensive care units have led to better out- comes after pancreatic resection and mortality rates are low while morbidity remains high ${ }^{10}$. Additionally, to further reduce morbidity and mortality to acceptable rates, centralization has been recommended for pancreatic resections. In high-volume specialized centers, mortality for the most common pancreatic resection, pancreaticoduodenectomy (PD), is now approximately $5 \%{ }^{11}$. However, morbidity remains high at a rate of $40-60 \%{ }^{10-12}$. Postoperative LOS after PD ranges from 14-20 days in various studies ${ }^{12-14}$. Complications, such as anastomotic leakage, pancreatic fistula, and delayed gastric emptying (DGE), are the main reasons for delayed recovery and frequent need for additional radiological or surgical interventions.

The benefit and safety of implementing ERAS programs in pancreatic surgery, including PD, has not yet been extensively studied. For this reason, a systematic review and meta-analysis of the available literature on ERAS pathways compared with traditional treatment in adult patients undergoing pancreatic surgery was performed with emphasis on PD, and time to recovery, length of postoperative hospital stay, morbidity, mortality, and readmissions were analyzed. 


\section{METHODS}

\section{Search strategy}

A search was conducted by two researchers (MC and AvdW) in January 2013 of Medline, Embase, Pubmed, CINAHL, and the Cochrane library spanning a period from January 1966 to December 2012 with language restricted to English, Dutch, or German using the search terms: "clinical pathway", "critical pathway", "enhanced recovery", and "fast track" and combinations of these with: "pancreas", "pancreatic", "Whipple", "pancreatectomy", "pancreaticoduodenectomy", using the Boolean operators AND and OR. Synonyms of all of these terms also were used in the search. Reference lists of relevant publications were assessed for additional references. Furthermore, bibliographies from other systematic reviews or meta-analyses on the subject were searched.

\section{Inclusion and exclusion criteria}

Studies meeting all of the following selection criteria were eligible for inclusion: (1) studies concerning adult patients undergoing major elective resectional surgery of the pancreas, (2) studies describing an enhanced recovery program with at least four different perioperative elements, (3) studies reporting at least the following outcome measures: LOS, postoperative morbidity and mortality, and readmission rates. Excluded were: (1) studies describing a single intervention in perioperative care rather than a bundle of elements combined in an enhanced recovery program, (2) studies describing emergency/nonelective or transplantation surgery, and (3) reviews.

These criteria were chosen following internal discussion in our research group as well as in the international ERAS group and are in keeping with earlier systematic reviews on ERAS in colonic surgery ${ }^{15,16}$. Practice was considered "ERAS" when at least four different items were included, covering the pre-, intra- and postoperative periods ${ }^{15,16}$. Table 2.1 shows different items used in ERAS protocols for pancreatic resections. The items are supported by varying levels of evidence.

\section{Systematic review and meta-analysis}

Systematic review was performed for articles on the implementation of an ERAS program for all pancreatic resections: distal pancreatectomy (DP), total pancreatectomy (TP), and PD. Subsequent meta-analysis was per- formed only on studies focusing on PD. 
Table $2.1 \quad$ Items in an ERAS protocol for pancreatic resection

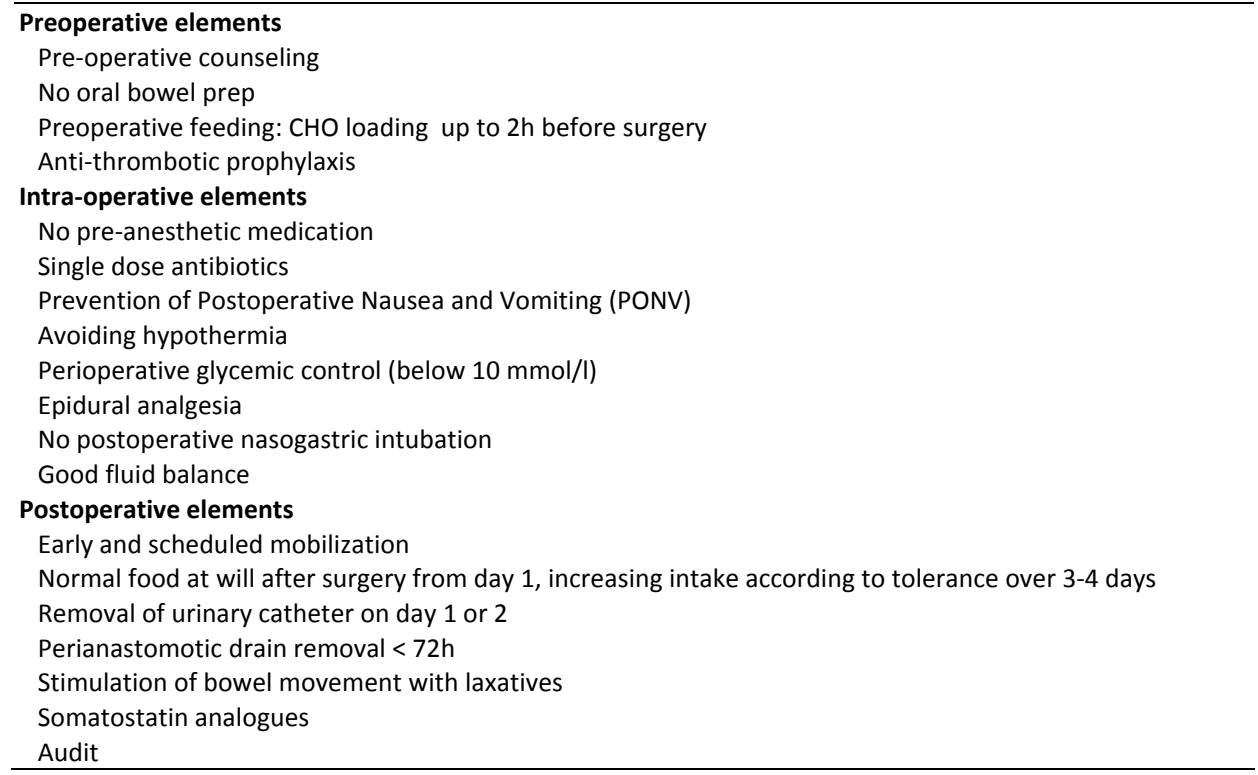

\section{Outcome measures}

The primary outcome measure of this systematic review was postoperative length of stay. Secondary outcome measures were time to recovery of normal function (criteria for functional recovery are: good pain control with oral analgesia, only taking solid food, no intravenous fluids, passage of stool and being independently mobile at preoperative level), overall postoperative complication rates, readmissions, and mortality. Secondary outcome measures also included delayed gastric emptying (DGE) and pancreatic fistulas in patient groups undergoing pancreaticoduodenectomy. Pancreatic fistula and delayed gastric emptying were defined according to the International Study Group of Pancreatic Surgery (ISGPS) ${ }^{17,18}$. Additionally, total hospital costs were analyzed if available.

\section{Study selection and data selection}

The two researchers identified and selected citations from the search independently. In case of doubt about inclusion, a third reviewer was consulted (RvD) until consensus was reached. Data from included studies were extracted independently by the two researchers. Randomized as well as nonrandomized studies were eligible as long as they met the inclusion criteria. Methodological quality of the articles was assessed using the MINORS checklist (Methodological Index for Non-Randomized Studies) ${ }^{19}$. This instrument scores 8 methodological items for noncomparative studies and an additional 4 criteria for comparative studies with a maximum score of 24 points for 
comparative and a maximum score of 16 points for noncomparative studies. Missing data were obtained by contacting the authors of the respective studies.

The following variables were extracted from the articles: number and age of patients in the study group and historical control groups if available, type of surgery, discharge criteria, and data on recovery to normal function (if available), duration of follow-up, postoperative length of hospital stay, complications, mortality, readmissions, and adherence to protocol. Furthermore, for each included article an assessment was made of the different ERAS program interventions. If available, hospital costs also were extracted from the articles.

\section{Statistical analysis}

Because searching the available literature produced no randomized, controlled trials, but only historical comparative studies, the MOOSE checklist for meta-analysis of observational studies was used to assess the possibility for conducting a metaanalysis $^{20}$. When studies focusing on PD were considered sufficiently clinically homogeneous according to the MOOSE consensus statement checklist, data were pooled and meta-analyzed using the statistical software package StatsDirect ${ }^{21}$. Metaanalysis of four comparative studies with historical controls that focused on PD was performed for morbidity, mortality, and readmissions. Meta-analysis of the incidence of complications, including pancreatic fistula and delayed gastric emptying, was performed in three studies addressing pancreaticoduodenectomy. For binary end points, risk differences and $95 \%$ confidence intervals were calculated using random effects models (Der-Simonian-Laird). Pooled risk differences were tested for statistical significance using Chi-square tests and a significance level of 0.05 . Heterogeneity was expressed in terms of $\mathrm{I}^{2}$. Results were presented graphically using Forest plots. To assess the impact of the different studies on the results, a sensitivity analysis was performed. In this sensitivity analysis, two different factors were explored: methodological quality of the studies and number of ERAS items used in a study protocol. Potential publication bias was explored using a funnel plot and Egger's test for risk of bias ${ }^{22}$.

\section{RESULTS}

\section{Selected articles and characteristics}

The results of the literature search and study selection are summarized in Figure 2.1. No randomized, controlled trials were available for inclusion. Full-text versions could not be retrieved of two articles despite contact with the authors of both articles. One relevant abstract was never published as a full paper and the original data could not be obtained through the author ${ }^{23}$. 


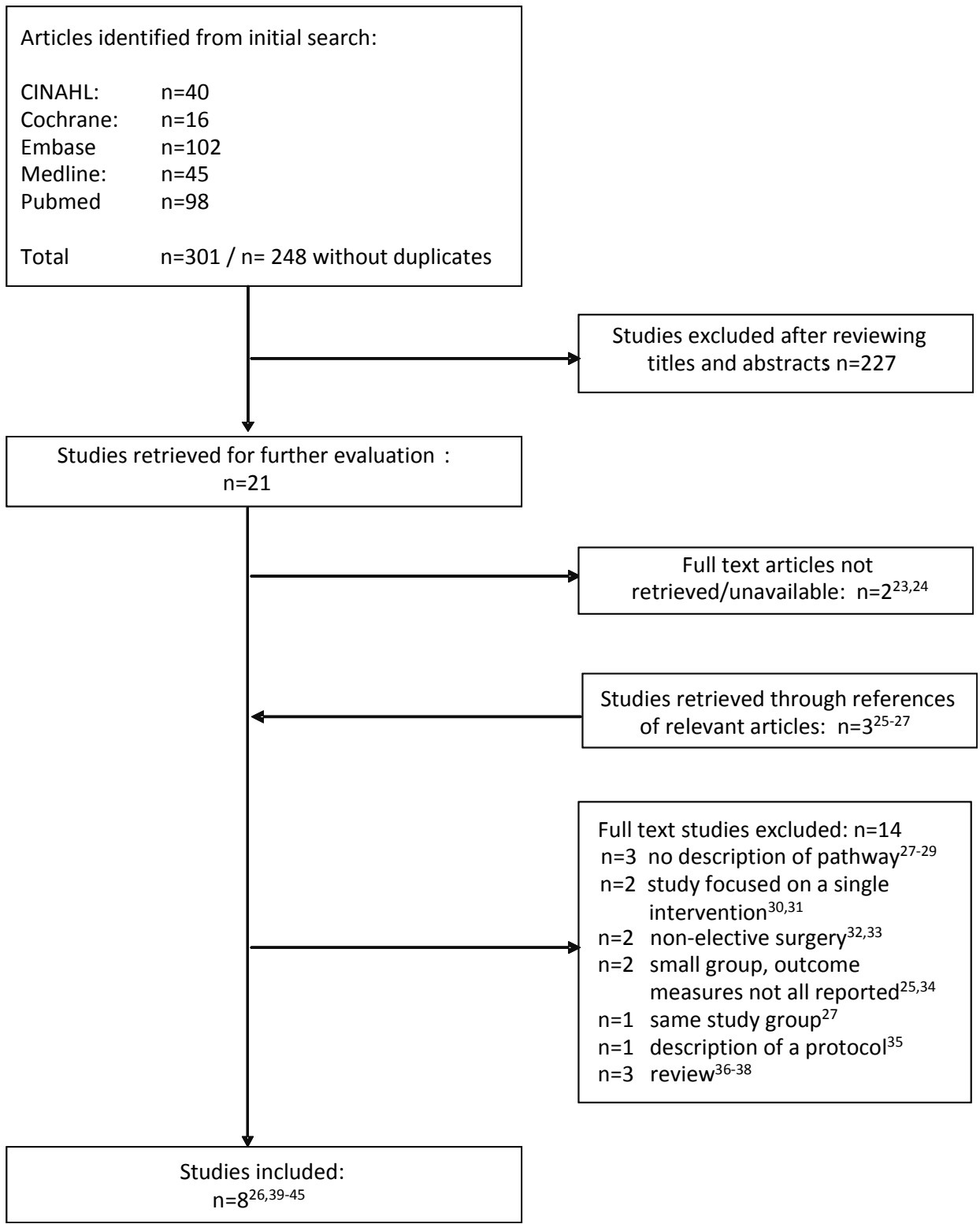

Figure 2.1 Study selection 
The author of the second article could not provide us with the original article published in Rozhledy V Chirurgii ${ }^{24}$, but mentioned that the same data were published in German elsewhere ${ }^{25}$. Subsequently, we excluded this article because not all outcome parameters were reported, there was no clear description of the pathway, and the study group was limited to 12 patients with no control group. Eight studies were finally included in this review: five comparative studies with historical controls studies, two retrospective case series, and one prospective case series. Characteristics of these seven studies are shown in Table 2.2. Five studies included only patients undergoing pancreaticoduodenectomy ${ }^{26,39-42}$, one study included only patients undergoing distal pancreatectomy ${ }^{43}$, and two studies included patients undergoing different forms of pancreatectomy (distal, central or total pancreatectomy, pylorus-pre-serving pancreatic head resections, segmental resections, or pancreatico-duodenectomy ${ }^{44,45}$. All studies included a consecutive series of patients with a follow-up of 30 days. The MINORS score of the comparative studies was on average 13.4, and the two retrospective case studies and one prospective case series were rated with scores of 12,13 , and 14 respectively (Table 2.2).

Six studies contained clear descriptions of an ERAS program with at least four different protocol elements. Study protocols of the two articles that did not describe the full ERAS program were retrieved from the authors. An assessment of the different protocol elements was made for each study (Supplementary Table S2.1). The number of protocol elements used in these study programs ranged from 9 to 14 with an average of 13 elements. All studies described pre-, peri-, and postoperative interventions, but study protocols differed slightly.

\section{Primary and secondary outcome measures}

\section{Systematic review}

For patients managed in an ERAS program, postoperative length of hospital stay (LOS) decreased significantly in four studies with a comparative design (Table 2.3). These studies included PDs as well as total pancreatectomies and distal pancreatectomies. Vanounou et al. ${ }^{26}$ did not find a difference between the study group and the control group in LOS. Three studies reported predefined discharge criteria and data on recovery to normal function ${ }^{39,42,44}$. However, only one of them reported exactly when the predefined discharge criteria were met or if there were any discrepancies between achieving functional recovery and actual discharge ${ }^{42}$.

A summary of total hospital costs calculated in four different studies is shown in Table $2.4^{26,40,41,43}$ Three of these studies included PDs; one study included distal pancreatectomies. All studies reported a decrease in total hospital costs after implementing a clinical pathway, and this decrease was significant in the three studies focusing on $P D^{26,40,41}$. 
Table 2.2 Study characteristics and quality assessment

\begin{tabular}{|c|c|c|c|c|c|c|c|c|}
\hline \multirow[t]{2}{*}{ Study } & \multirow{2}{*}{$\begin{array}{l}\text { Type of } \\
\text { surgery }\end{array}$} & \multirow[t]{2}{*}{ Study design } & \multirow{2}{*}{$\begin{array}{l}\text { No of } \\
\text { patients in } \\
\text { study/ } \\
\text { control } \\
\text { group }\end{array}$} & \multirow{2}{*}{$\begin{array}{c}\text { Consecutive } \\
\text { series of } \\
\text { patients }\end{array}$} & \multirow{2}{*}{$\begin{array}{l}\text { Follow- } \\
\text { up } \\
\text { (days) }\end{array}$} & \multicolumn{2}{|c|}{ Mean age (years) } & \multirow{2}{*}{$\begin{array}{l}\text { MINORS } \\
\text { score }\end{array}$} \\
\hline & & & & & & ERAS & control & \\
\hline $\begin{array}{l}\text { Balzano } \\
\text { et al. } 2008^{39}\end{array}$ & PPPD & $\begin{array}{l}\text { Comparative } \\
\text { study with } \\
\text { historical } \\
\text { controls }\end{array}$ & $252 / 252$ & Yes & 30 & 64 & 63 & $13 / 24$ \\
\hline $\begin{array}{l}\text { Porter } \\
\text { et al. } 2000^{40}\end{array}$ & $P D, T P$ & $\begin{array}{c}\text { Comparative } \\
\text { study with } \\
\text { historical } \\
\text { controls }\end{array}$ & $80 / 68$ & Yes & 30 & 60 & 62 & $12 / 24$ \\
\hline $\begin{array}{l}\text { Kennedy } \\
\text { et al. } 2009^{43}\end{array}$ & DP & $\begin{array}{l}\text { Comparative } \\
\text { study with } \\
\text { historical } \\
\text { controls }\end{array}$ & $72 / 40$ & Yes & 30 & 63.4 & 53.6 & $11 / 24$ \\
\hline $\begin{array}{l}\text { Vanounou } \\
\text { et al. } 2007^{26}\end{array}$ & PD/PPPD & $\begin{array}{c}\text { Comparative } \\
\text { study with } \\
\text { historical } \\
\text { controls }\end{array}$ & $145 / 64$ & Yes & 30 & $\begin{array}{c}64 \\
\text { (median) }\end{array}$ & $\begin{array}{c}64 \\
\text { (median) }\end{array}$ & $15 / 24$ \\
\hline $\begin{array}{l}\text { Kennedy } \\
\text { et al. } 2007^{41}\end{array}$ & PD & $\begin{array}{l}\text { Comparative } \\
\text { study with } \\
\text { historical } \\
\text { controls }\end{array}$ & $91 / 44$ & Yes & 30 & 63.9 & 61.3 & $16 / 24$ \\
\hline $\begin{array}{l}\text { DiSebastiano } \\
\text { et al. } 2010^{44}\end{array}$ & $\begin{array}{l}\text { PD, TP, } \\
\text { DP }\end{array}$ & $\begin{array}{l}\text { Retrospective } \\
\text { case series }\end{array}$ & 145 & Yes & 30 & 65 & - & $12 / 16$ \\
\hline $\begin{array}{l}\text { Berberat } \\
\text { et al. } 2007^{45}\end{array}$ & $\begin{array}{c}\text { DP, PD, } \\
\text { DPPHR, } \\
\text { TP, SP }\end{array}$ & $\begin{array}{l}\text { Retrospective } \\
\text { case series }\end{array}$ & 255 & Yes & 30 & 59 & - & $13 / 16$ \\
\hline $\begin{array}{l}\text { Robertson } \\
\text { et al. } 2012^{42}\end{array}$ & PD, PPPD & $\begin{array}{l}\text { Prospective } \\
\text { case series }\end{array}$ & 50 & Yes & 30 & $\begin{array}{c}67 \\
\text { (median) }\end{array}$ & - & $14 / 16$ \\
\hline
\end{tabular}

PPPD: pyloruspreserving pancreaticoduodenectomy, PD: pancreaticoduodenectomy, TP: total pancreatictomy, DP: distal pancreatectomy, SP: segmental pancreatectomy, DPPHR: duodenum preserving pancreatic head resection

Three studies described adherence to protocol for different protocol elements ${ }^{39,42,45}$. In most patients, the nasogastric tube could be removed on the first postoperative day. However, it had to be reinserted in $11-15 \%$ of the patients ${ }^{39,45}$. Normal intake was achieved on median days 4-5, and mobilization was achieved according to protocol in approximately $70-85 \%$ of the patients ${ }^{39,42,45}$. Berberat et al. also performed uni- and multivariable analysis in which early removal of intra-abdominal drains, occurrence of first stool, normal food, and complete mobilization correlated significantly with early discharge. Furthermore, other patient specific predictors of early discharge were age $<60$ years, low ASA score, BMI $<25$, and presence of benign disease. Surgical factors contributing to early discharge were short operation time, blood loss $<1,000 \mathrm{ml}$, 
absence of blood transfusion, and early extubation. More extensive resections (Whipple, segmental resections) were negatively correlated with early discharge ${ }^{45}$.

Table 2.3 Postoperative outcome after implementation of a clinical pathway

\begin{tabular}{|c|c|c|c|c|c|}
\hline Study & $\begin{array}{l}\text { Type of } \\
\text { surgery }\end{array}$ & $\begin{array}{l}\text { Length of hospital } \\
\text { stay (days; study } \\
\text { group vs control) }\end{array}$ & $\begin{array}{l}\text { Morbidity (\%; } \\
\text { study group vs } \\
\text { control) }\end{array}$ & $\begin{array}{l}\text { Mortality (\%; } \\
\text { study group vs } \\
\text { control) }\end{array}$ & $\begin{array}{c}\text { Readmissions (\%; } \\
\text { study group vs } \\
\text { control) }\end{array}$ \\
\hline $\begin{array}{l}\text { Balzano } \\
\text { et al. } 2008^{39}\end{array}$ & PPPD & $\begin{array}{c}13(7-110) \text { vs } \\
15(7-102) \\
(p<0.001)\end{array}$ & $\begin{array}{c}47.2 \text { vs } 58.7 \\
(p=0.014)\end{array}$ & $\begin{array}{c}3.6 \text { vs } 2.8 \\
\text { (ns) }\end{array}$ & $\begin{array}{c}7.1 \text { vs } 6.3 \\
\text { (ns) }\end{array}$ \\
\hline $\begin{array}{l}\text { Porter } \\
\text { et al. } 2000^{40}\end{array}$ & $P D, T P$ & $\begin{array}{c}13.5 \text { vs } 16.4 \\
(p=0.001)\end{array}$ & $\begin{array}{c}24 \text { vs } 20 \\
\text { (ns) }\end{array}$ & $\begin{array}{c}1 \text { vs } 3 \\
\text { (ns) }\end{array}$ & $\begin{array}{l}9 \text { vs } 10 \\
\text { (ns) }\end{array}$ \\
\hline $\begin{array}{l}\text { Kennedy } \\
\text { et al. } 2009^{43}\end{array}$ & DP & $\begin{array}{l}6.7 \text { vs } 10.2 \\
(p=0.037)\end{array}$ & $\begin{array}{c}15.7 \text { vs } 37.5 \\
\text { (ns) }\end{array}$ & $\begin{array}{l}1.1 \text { vs } 2.3 \\
\text { (ns) }\end{array}$ & $\begin{array}{c}7 \text { vs } 25 \\
(p=0.027)\end{array}$ \\
\hline $\begin{array}{l}\text { Vanounou } \\
\text { et al. } 2007^{26}\end{array}$ & PD/PPPD & 8 vs 8 (ns) & $\begin{array}{c}54 \text { vs } 62 \\
\text { (ns) }\end{array}$ & $\begin{array}{l}1.4 \text { vs } 1.6 \\
\text { (ns) }\end{array}$ & $\begin{array}{c}9 \text { vs } 6 \\
\text { (ns) }\end{array}$ \\
\hline $\begin{array}{l}\text { Kennedy } \\
\text { et al. } 2007^{41}\end{array}$ & PD & $\begin{array}{c}7 \text { vs } 13 \\
(p<0.0001)\end{array}$ & $\begin{array}{c}37 \text { vs } 44 \\
\text { (ns) }\end{array}$ & $\begin{array}{l}1.1 \text { vs } 2.3 \\
\text { (ns) }\end{array}$ & $\begin{array}{c}7.7 \text { vs } 7 \\
\text { (ns) }\end{array}$ \\
\hline $\begin{array}{l}\text { DiSebastiano } \\
\text { et al. } 2010^{44}\end{array}$ & $P D, T P, D P$ & $10(6-69)$ & 38.6 & 2.7 & 6.2 \\
\hline $\begin{array}{l}\text { Berberat } \\
\text { et al. } 2007^{45}\end{array}$ & $\begin{array}{c}\text { DP, PD, } \\
\text { DPPHR, TP, } \\
\text { SP }\end{array}$ & $10(4-115)$ & 24.7 & 2 & 3.5 \\
\hline $\begin{array}{l}\text { Robertson } \\
\text { et al. } 2012^{42}\end{array}$ & PD, PPPD & $10(8-17)$ & 46 & 4 & 4 \\
\hline
\end{tabular}

PPPD: pyloruspreserving pancreaticoduodenectomy, PD: pancreaticoduodenectomy, TP: total pancreatectomy, DP: distal pancreatectomy, SP: segmental pancreatectomy, DPPHR: duodenum preserving pancreatic head resection, ns: no significant differences, $p<0.05$ is considered significant

Table 2.4 Total hospital costs

\begin{tabular}{lccccc}
\hline Study & Type of surgery & $\begin{array}{c}\text { No of patients in } \\
\text { control/study } \\
\text { group }\end{array}$ & Costs pre-pathway & $\begin{array}{c}\text { Costs post- } \\
\text { pathway }\end{array}$ & P-value \\
\hline $\begin{array}{l}\text { Porter } \\
\text { et al. } 2000^{40}\end{array}$ & PD, TP & $68 / 80$ & $\$ 47,515$ & $\$ 36,627$ & 0.003 \\
$\begin{array}{l}\text { Kennedy } \\
\text { et al. } 2009^{43}\end{array}$ & DP & $40 / 72$ & $\$ 26,393 \pm 4719$ & $\$ 22,806 \pm 1300$ & ns \\
$\begin{array}{l}\text { Vanounou } \\
\text { et al. } 2007^{26}\end{array}$ & PD/PPPD & $64 / 145$ & $\$ 28,886$ & $\$ 23,344$ & $<0.001$ \\
$\begin{array}{l}\text { Kennedy } \\
\text { et al. } 2007^{41}\end{array}$ & PD & $44 / 91$ & $\$ 240,242 \pm 32,490$ & $\$ 126,566 \pm 4883$ & $<0.001$ \\
\hline
\end{tabular}

PPPD: pyloruspreserving pancreaticoduodenectomy, PD: pancreaticoduodenectomy, TP: total pancreastectomy, DP: distal pancreatectomy, SP: segmental pancreatectomy, DPPHR: duodenum preserving pancreatic head resection, ns: no significant differences, $p<0.05$ is considered significant 


\section{Meta-analysis}

Meta-analysis was performed on four comparative studies focusing on PD. One study reported on 148 patients of whom 12 patients underwent TP and $136 \mathrm{PD}^{40}$. Because we were unable to obtain specific data of only the 136 PDs, despite repeated attempts to contact the author, we decided to perform the meta-analysis with these four articles on PD, including 984 PDs in total and 12 TPs (i.e. we assumed the 12 TPs would not affect the main outcome). Meta-analysis for mortality did not show a difference between the study group and the control group [risk difference (95\% confidence interval $(\mathrm{Cl}) 0.2 \%$ $(-1.7$ to 2.1$) \mathrm{I}^{2}=0 \%, \mathrm{p}=0.83$; Figure 2.2 ]. For readmission rates, meta-analysis did not show a significant difference [risk difference $(95 \% \mathrm{Cl}) 0.8 \%(-2.5$ to 4.1$) \mathrm{I}^{2}=0 \%, \mathrm{p}=0.64$; Figure 2.3]. Regarding overall morbidity, there was a consistent difference in favor of the ERAS group. The reported percentage of postoperative morbidity was lower in the ERAS group [absolute risk difference $(95 \% \mathrm{Cl}) 8.2(2-14.5) \mathrm{I}^{2}=0 \%, \mathrm{p}=0.008$ ]. The number needed to treat (NNT) was approximately 10 (Figure 2.4 ).

Risk difference meta-analysis plot [random effects]

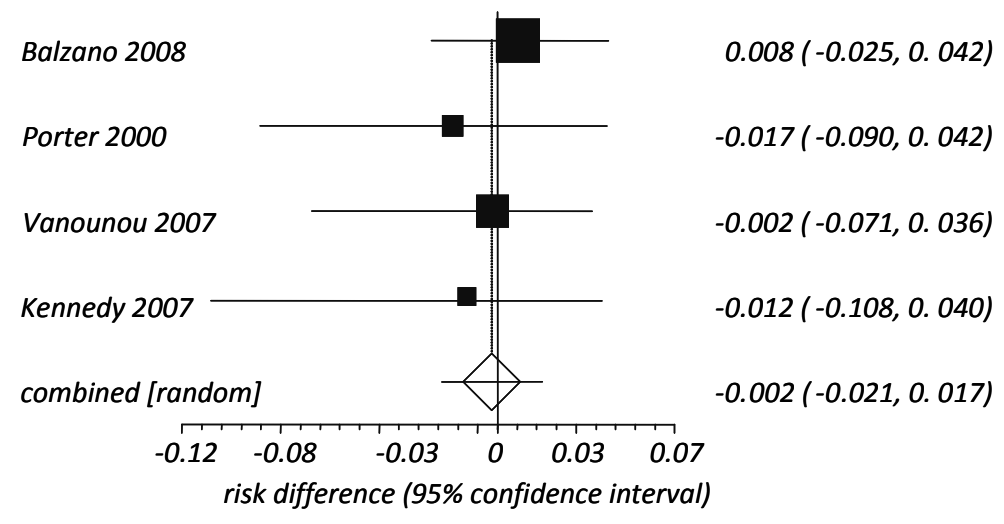

Favors experimental

Favors control

Figure 2.2 Forest plot of comparison: Mortality 
Risk difference meta-analysis plot [random effects]

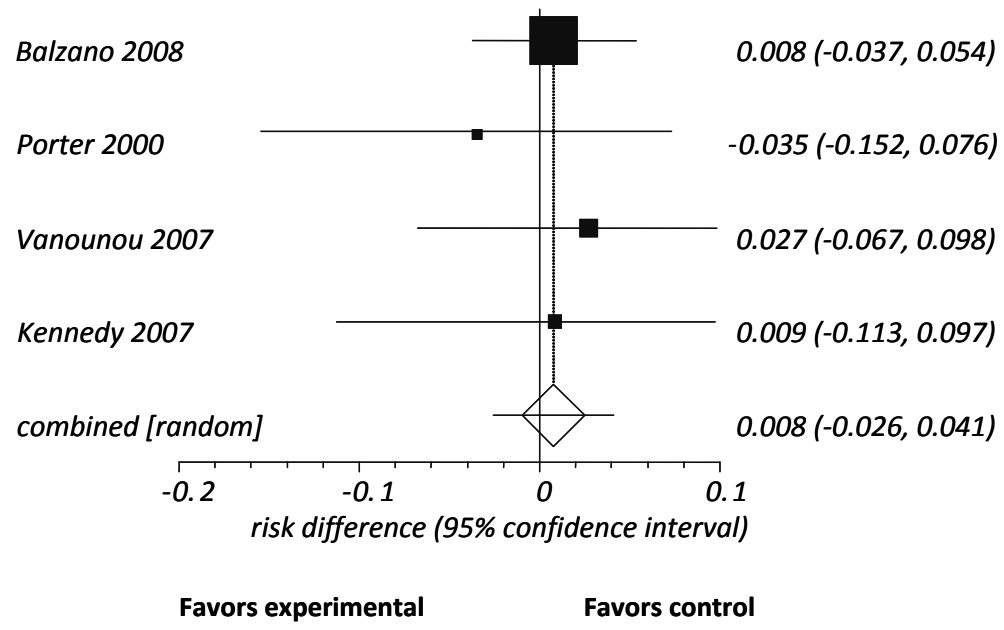

Figure 2.3 Forest plot of comparison: Readmissions

Risk difference meta-analysis plot [random effects]

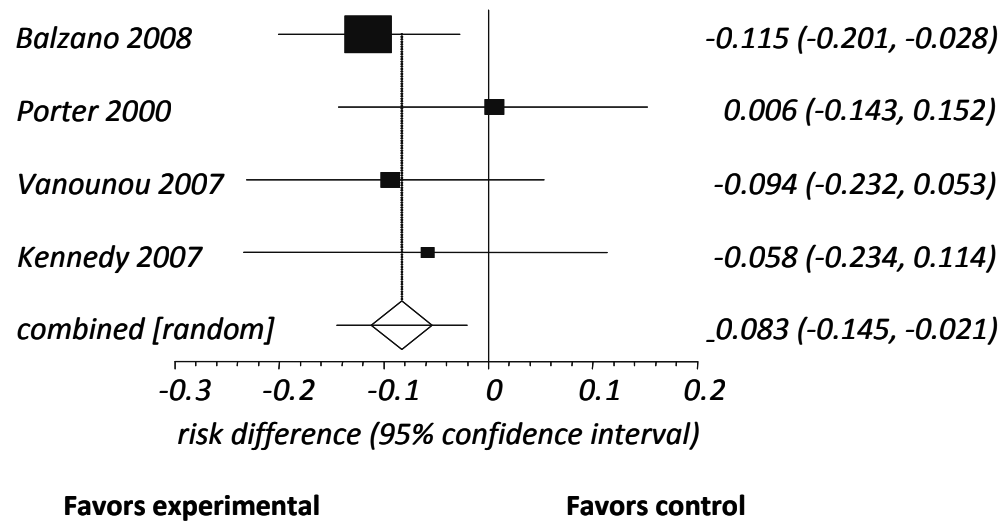

Figure $2.4 \quad$ Forest plot of comparison: Morbidity

Sensitivity analysis was performed to assess impact of methodological quality and number of ERAS protocol items on outcome. The overall relative risk was $0.83(95 \% \mathrm{Cl}$ 0.73-0.95), indicating complications were consistently lower in the ERAS groups compared with controls. This result depends mainly on the studies of good 
methodological quality (MINOR score of 13 or more) ${ }^{26,39,41}$, as analysis of these studies only shows a comparable relative risk of 0.82 (95\% $\mathrm{Cl} 0.72-0.94)$. Also, when excluding studies with less than 13 protocol items ${ }^{39,40}$, sensitivity analysis shows a relative risk comparable to the overall relative risk 0.85 (95\% $\mathrm{Cl} 0.69-1.06)$.

To assess the presence of bias in this meta-analysis, a funnel plot was constructed. Egger et al. ${ }^{20}$ proposed a test for asymmetry of the funnel plot; the trials' effect estimate is set out against its sample size. Figure 2.5 shows a symmetrical deviation of the different studies. The calculated $p$ value is 0.27 , indicating the chance of bias in this meta-analysis, including only studies focusing on PD is low.

Complication rates of pancreatic fistula and DGE after PD ranged from 2 to $26 \%$ and 7-25\% respectively in the included studies. Meta-analysis of three studies focusing on PDs and reporting incidence rates of PF and DGE showed that the incidence of pancreatic fistula and DGE was not significantly different between the intervention and control groups (data not shown) ${ }^{39-41}$.

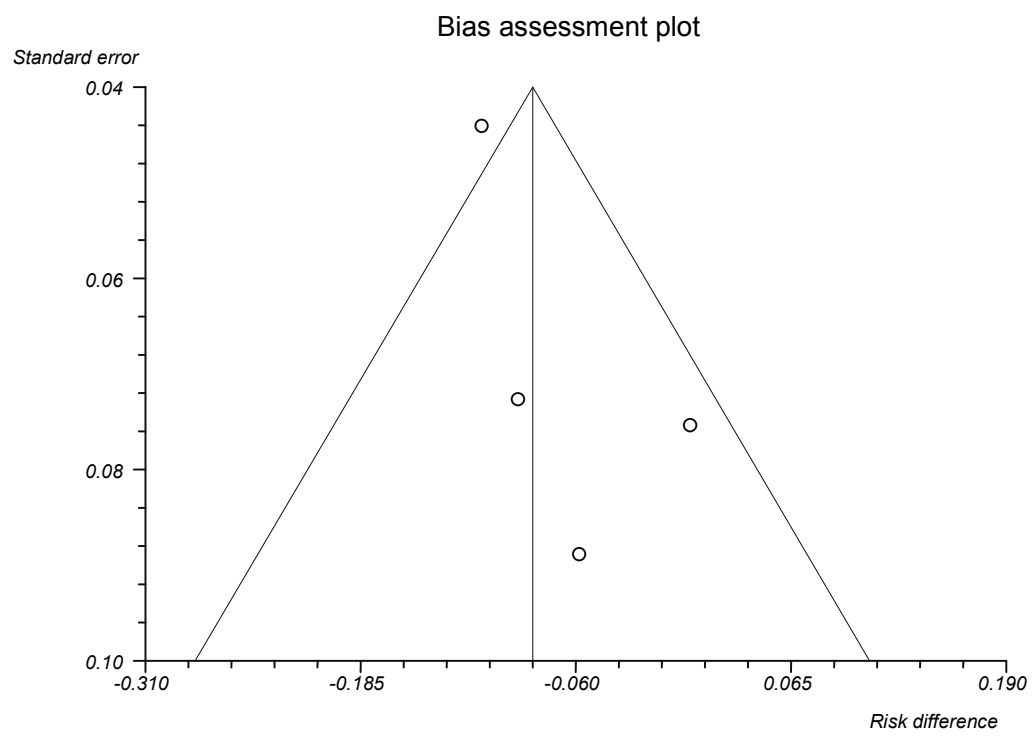

Egger: bias $=1,847835(95 \% \mathrm{Cl}=-3,564737$ to 7,260407$) \mathrm{P}=0,2796$

Figure 2.5 Funnel plot of 4 studies included in the meta-analysis 


\section{DISCUSSION}

This systematic review shows that perioperative management based on an ERAS program in pancreatic resection may help shorten hospital length of stay without compromising morbidity and mortality. This seemed to apply to distal pancreatectomy, total pancreatectomy, and PD. Because these procedures differ considerably as to consequences for perioperative care, meta-analysis was only performed for those studies that focused on PD, as this is the most common pancreatic resection. This metaanalysis of four studies on ERAS programs in PD showed that morbidity rates were significantly lower for patients in the ERAS study group compared with those treated in a traditional care pathway. This effect remained unaltered when studies of lower methodological quality or studies, including less than 13 elements in the ERAS protocol, were excluded. After meta- analysis, readmission rates were not significantly higher in the ERAS group. One study reported a non-significant $1 \%$ higher mortality rate in the ERAS group $^{39}$. Also, on meta- analysis of the four studies addressing PDs, mortality was not significantly different. This is in keeping with another meta-analyses on ERAS programs in colonic surgery in which no higher mortality was found ${ }^{15}$. Additionally, hospital costs were significantly lower in three of the four studies that reported a costeffectiveness analysis. Unfortunately, in most studies no data on functional recovery or predefined discharge criteria were available. Accordingly, no conclusion could be drawn on the assumed positive effects of an ERAS program on functional recovery.

A previous systematic review concluded that there is little evidence to support the benefits of ERAS programs for pancreatic surgery ${ }^{36}$. This review included only three studies and concluded that an ERAS program in pancreatic surgery was safe but that morbidity rates were higher in the ERAS group. However, this conclusion was not in accordance with the results presented, because the source data from the studies showed an "equal morbidity" between study and control group.

A limitation of this review is that no randomized, controlled trials were available for inclusion. Only a limited number of randomized, controlled trials have been conducted comparing ERAS programs with traditional care ${ }^{15,46}$, mostly in elective colorectal surgery. No such trials have been conducted in HPB surgery. This is probably due to the fact that randomized controlled trials are difficult to organize for multimodal recovery programs under greatly varying conditions. Problems are very likely to be encountered with blinding and various interventions and professionals are involved. Several protocol elements of an ERAS program already have become standard practice in many hospitals during the last decade (e.g. thromboprophylaxis, prevention of hypothermia with Bair Huggers, early mobilization). This raises ethical concerns with respect to randomization, because patients in the control group may not receive specific evidence-based interventions.

It must be noted that the introduction of an ERAS program in the two included studies by Kennedy et al. took place after two experienced pancreatic surgeons moved in from 
a different hospital. In parallel with the implementation of the ERAS program, surgical volume per month almost tripled. These facts may have contributed to improved outcome regardless of the implementation of the ERAS program.

In line with this, one could argue that any positive effects of ERAS implementation are not attributable to the ERAS program itself but to changing secular trends with respect to lowering LOS. However, a recent national implementation study in the Netherlands of colorectal surgery looking at the effects of ERAS implementation in more than 30 hospitals nationwide showed that hospital LOS decreased by 3 days in 1 year in addition to any such secular trends ${ }^{47}$.

After a pancreaticoduodenectomy, the incidence of specific complications, such as DGE and pancreatic fistula, varies from 13 to $57 \%^{10,13,48,49}$ and from 2 to $15 \%$, respectively ${ }^{10,13,50-52}$. In the present review, the incidence of these complications tended to be lower in the ERAS group, but meta-analysis in patients undergoing pancreaticoduodenectomy did not show statistical significance.

The included studies were either retrospective or prospective case series or comparative studies based on historical controls. It is difficult to extract conclusive evidence from such studies. The use of historical controls also led to variable methodological quality of the different studies, as rated with the MINORS criteria.

Another topic of concern in the investigation of perioperative pathways is the fact that distinct studies use different study protocols incorporating a variety of elements and some protocols might include elements that are more conservative than others. For instance, postoperative feeding started from postoperative days 1-2 in some studies $^{44,45}$, whereas other studies started with liquid intake first with a gradual increase from clear to full liquids and solid food from days $3-4^{26,39,41-43}$. Unfortunately, it is still unclear which element is most important in perioperative care in pancreatic resections and henceforth we cannot draw hard conclusions about that. However, in a recent paper a multivariable analysis of care elements influencing LOS in 2,485 patients undergoing colonic surgery ${ }^{47}$, cessation of IV fluids, mobilization on post- operative day 1 , and postoperative administration of laxatives (magnesium oxide) were associated with shortened LOS. In this way, ERAS programs may accelerate restoration of gut motility. In PD, this might help to lower the incidence of DGE, which also was shown in one of the included studies ${ }^{39}$.

Most studies do not investigate adherence to the protocol, so it remains unclear what the actual clinical difference in perioperative treatment is between study and control group. Problems with protocol adherence have already been described in a large international study in ERAS colon surgery by Maessen et al. ${ }^{53}$. Reporting of adherence to protocol should be a standard item in studies describing the ERAS implementation. Policies to improve postoperative protocol adherence in particular should be considered, for instance reorganization of surgical wards, continuous education of nurses and staff, and the introduction of self-reporting diaries ${ }^{53}$. A study of Ahmed et al. showed that an overall protocol compliance of $77 \%$ compared with $88 \%$ compliance 
in patients participating in a clinical trial does not negatively affect outcome ${ }^{54}$. Particularly, low compliance to preoperative carbohydrate loading and early fluid and diet introduction postoperative was observed. This study shows that it is rather the combination of items that determines outcome and that a reasonable adherence is acceptable.

On a further note, the consistency between criteria defining minor and major complications after pancreatic surgery in the included studies is limited. Therefore, comparing morbidity between centers is difficult. Only one study reported complications according to a validated classification scheme (Clavien-Dindo classification), grading complications based on the use of therapeutic interventions ${ }^{26}$. A suggestion would be to use a composite end point ${ }^{55}$, which would reduce the required sample sizes for studies and would improve objectivity and comparability.

Clearly, the studies included in this review are all subject to different forms of bias and heterogeneity in study groups as well as ERAS programs. This implies that it is difficult to draw hard conclusions on the effectiveness of ERAS programs in pancreatic surgery. Therefore, the ERAS group recently conceived perioperative guidelines focused only on pancreaticoduodenectomy ${ }^{56}$. The feasibility of implementing these guidelines will be studied in the ERAS group in a multicenter, prospective consecutive series of patients undergoing pancreaticoduodenectomy (www.erassociety.org).

In summary, although the available evidence is still limited, the present systematic review suggests that implementation of an ERAS program in pancreatic resections, particularly PD, is feasible and safe. Implementation of an ERAS program in pancreatic resections may contribute to a shorter hospital stay and does not seem to compromise outcome measures, such as morbidity, mortality, and readmissions. Future studies should report on predefined discharge criteria and time to functional recovery to assess whether actual postoperative recovery is in fact accelerated. 


\section{REFERENCES}

1. Kehlet $\mathrm{H}$. Multimodal approach to control postoperative pathophysiology and rehabilitation. $\mathrm{Br} J$ Anaesth 1997;78:606-617.

2. Kehlet H, Wilmore DW. Multimodal strategies to improve surgical outcome. Am J Surg 2002;183:630641.

3. Barbieri A, Vanhaecht K, Van Herck P, Sermeus W, Faggiano F, Marchisio S, Panella M. Effects of clinical pathways in the joint replacement: a meta-analysis. BMC Med 2009;7:32.

4. Arsalani-Zadeh R, ElFadl D, Yassin N, MacFie J Evidence-based review of enhancing postoperative recovery after breast surgery. Br J Surg 2011;98:181-196.

5. Podore PC, Throop EB. Infrarenal aortic surgery with a 3-day hospital stay: a report on success with a clinical pathway. J Vasc Surg 1999;29:787-792.

6. Brustia P, Renghi A, Gramaglia L, Porta C, Cassatella R, De Angelis R, Tiboldo F. Mini-invasive abdominal aortic surgery. Early recovery and reduced hospitalization after multidisciplinary approach. J CardiovasC Surg 2003;44:629-635.

7. Wasowicz-Kemps DK, Bliemer B, Boom FA, de Zwaan NM, van Ramshorst B. Laparoscopic gastric banding for morbid obesity: outpatient procedure versus overnight stay. Surg Endosc 2006;20: 1233-1237.

8. McCarty TM, Arnold DT, Lamont JP, Fisher TL, Kuhn JA. Optimizing outcomes in bariatric surgery: outpatient laparoscopic gastric bypass. Ann Surg 2005;242:494-498.

9. Kirsh EJ, Worwag EM, Sinner M, Chodak GW. Using outcome data and patient satisfaction surveys to develop policies regarding minimum length of hospitalization after radical prostatectomy. Urology 2000;56:101-106.

10. DeOliveira ML, Winter JM, Schafer M, Cunningham SC, Cameron JL, Yeo CJ, Clavien PA. Assessment of complications after pancreatic surgery: a novel grading system applied to 633 patients undergoing pancreaticoduodenectomy. Ann Surg 2006;244:931-937.

11. de Wilde RF, Besselink MG, van der Tweel I, de Hingh IH, van Eijck CH, Dejong CH, Porte RJ, Gouma DJ, Busch OR, Molenaar IQ; Dutch Pancreatic Cancer Group. Impact of nationwide centralization of pancreaticoduodenectomy on hospital mortality. Br J Surg 2012;99:404-410.

12. Gouma DJ1, van Geenen RC, van Gulik TM, de Haan RJ, de Wit LT, Busch OR, Obertop H. Rates of complications and death after pancreaticoduodenectomy: risk factors and the impact of hospital volume. Ann Surg 2000;232:786-795.

13. Yeo CJ, Cameron JL, Sohn TA, Lillemoe KD, Pitt HA, Talamini MA, Hruban RH, Ord SE, Sauter PK, Coleman J, Zahurak ML, Grochow LB, Abrams RA. Six hundred fifty consecutive pancreaticoduodenectomies in the 1990s: pathology, complications, and outcomes. Ann Surg 1997;226:248-257.

14. Richter A, Niedergethmann M, Sturm JW, Lorenz D, Post S, Trede M. Long-term results of partial pancreaticoduodenectomy for ductal adenocarcinoma of the pancreatic head: 25-year experience. World J Surg 2003;27:324-329.

15. Varadhan KK, Neal KR, Dejong CH, Fearon KC, Ljungqvist O, Lobo DN. The enhanced recovery after surgery (ERAS) pathway for patients undergoing major elective open colorectal surgery: a meta-analysis of randomized controlled trials. Clin Nutr 2010;29: 434-440.

16. Wind J, Polle SW, Fung Kon Jin PH, Dejong CH, von Meyenfeldt MF, Ubbink DT, Gouma DJ, Bemelman WA; Laparoscopy and/or Fast Track Multimodal Management Versus Standard Care (LAFA) Study Group; Enhanced Recovery after Surgery (ERAS) Group. Systematic review of enhanced recovery programmes in colonic surgery. Br J Surg 2006;93:800-809.

17. Wente MN, Bassi C, Dervenis C, Fingerhut A, Gouma DJ, Izbicki JR, Neoptolemos JP, Padbury RT, Sarr MG, Traverso LW, Yeo CJ, Büchler MW. Delayed gastric emptying (DGE) after pancreatic surgery: a suggested definition by the international study group of pancreatic surgery (ISGPS). Surgery 2007;142:761-768.

18. Bassi C, Dervenis C, Butturini G, Fingerhut A, Yeo C, Izbicki J, Neoptolemos J, Sarr M, Traverso W, Buchler M; International Study Group on Pancreatic Fistula Definition. Postoperative pancreatic fistula: an international study group (ISGPF) definition. Surgery 2005;138:8-13. 
19. Slim K, Nini E, Forestier D, Kwiatkowski F, Panis Y, Chipponi J. Methodological index for non-randomized studies (minors): development and validation of a new instrument. ANZ J Surg 2003;73:712-716

20. Stroup DF, Berlin JA, Morton SC, Olkin I, Williamson GD, Rennie D, Moher D, Becker BJ, Sipe TA, Thacker SB. Meta-analysis of observational studies in epidemiology: a proposal for reporting. Meta-analysis of obser- vational studies in epidemiology (MOOSE) group. JAMA 2000;283:2008-2012.

21. Freemantle N. StatsDirect-statistical software for medical research in the 21st century. BMJ 2000;321:1536.

22. Egger M. Bias in meta-analysis detected by a simple, graphical test. BMJ 1997;13:629-634.

23. Chaudhary A, Parvez A, Singh AP Early discharge after pancreaticoduodenectomy-fair or foul? Conference: 39th annual meeting of the American pancreatic association Chicago, IL. November 7-8, 2008. Pancreas 2008;37:464.

24. Wichmann MW, Roth $\mathrm{M}$, Jauch $\mathrm{KW}$, Bruns CJ. A prospective clinical feasibility study for multimodal "fast track" rehabilitation in elective pancreatic cancer surgery. Rozhl Chir 2006;85:169-175.

25. Bruns C, Wichmann MW et al. Fast track pancreatic cancer surgery. Chirurgische Praxis 2007;67: 203-210.

26. Vanounou T, Pratt W, Fischer JE, Vollmer CM Jr, Callery MP. Deviation-based cost modeling: a novel model to evaluate the clinical and economic impact of clinical pathways. J Am Coll Surg 2007;204: 570-579.

27. Vollmer CM Jr, Pratt W, Vanounou T, Maithel SK, Callery MP. Quality assessment in high-acuity surgery: volume and mortality are not enough. Arch Surg 2007;142:371-380.

28. Balcom JH 4th, Rattner DW, Warshaw AL, Chang Y, Fernandez-del Castillo C. Ten-year experience with 733 pancreatic resections: changing indications, older patients, and decreasing length of hospitalization. Arch Surg 2001;136:391-398.

29. French JJ, Mansfield SD, Jaques K, Jaques BC, Manas DM, Charnley RM. Fast-track management of patients undergoing proximal pancreatic resection. Ann R Coll Surg Engl 2009;91:201-204.

30. Kow AW, Chan SP, Earnest A, Chan CY, Lim K, Chong SY, Lim KH, Ho CK, Chew SP, Liau KH. Striving for a better operative outcome: 101 pancreaticoduodenectomies. HPB (Oxford) 2008;10:464-471.

31. Pratt WB, Steinbrook RA, Maithel SK, Vanounou T, Callery MP, Vollmer CM Jr. Epidural analgesia for pancreatoduodenectomy: a critical appraisal. J Gastrointest Surg 2008;12:1207-1220.

32. Fukase M, Shimamura H, Takeda K. Introduction of the critical path for pancreaticoduodenectomy taking in the concept of probiotics. Conference: joint 40th anniversary meeting of American pancreatic association and Japan pancreas society Honolulu, HI United States. November 4-11, 2009. Pancreas 2009;38:997.

33. Little $A B$, Whipple TW. Clinical pathway implementation in the acute care hospital setting. J Nurs Care Qual 1996;11:54-61.

34. Pratt WB, Vollmer CM, Callery MP. Outcomes in pancreatic resection are negatively influenced by preoperative hospitalization. HPB 2009;11:57-65

35. Burden $\mathrm{S}$, et al. Study protocol: preoperative nutrition in patients undergoing gastrointestinal surgery. Cochrane Database Syst Rev. 2012;14;11;CD008879

36. Ypsilantis E, Praseedom RK. Current status of fast-track recovery pathways in pancreatic surgery. $J$ Pancreas 2009;10:646-650.

37. Spelt L, Ansari D, Sturesson C, Tingstedt B, Andersson R. Fast-track programmes for hepatopancreatic resections: where do we stand? HPB 2011;13:833-838.

38. Hall TC, Dennison AR, Bilku DK, Metcalfe MS, Garcea G. Enhanced recovery programmes in hepatobiliary and pancreatic surgery: a systematic review. Ann R Coll Surg Engl 2012;94:318-326.

39. Balzano G, Zerbi A, Braga M, Rocchetti S, Beneduce AA, Di Carlo V. Fast-track recovery programme after pancreaticoduodenectomy reduces delayed gastric emptying. Br J Surg 2008;95:1387-1393.

40. Porter GA, Pisters PW, Mansyur C, Bisanz A, Reyna K, Stanford P, Lee JE, Evans DB. Cost and utilization impact of a clinical pathway for patients undergoing pancreaticoduodenectomy. Ann Surg Oncol 2000;7:484-489.

41. Kennedy EP, Rosato EL, Sauter PK, Rosenberg LM, Doria C, Marino IR, Chojnacki KA, Berger AC, Yeo CJ. Initiation of a critical pathway for pancreaticoduodenectomy at an academic institution-the first step in multidisciplinary team building. J Am Coll Surg 2007;204:917-923. 
42. Robertson N, Gallacher PJ, Peel N, Garden OJ, Duxbury M, Lassen K, Parks RW. Implementation of an enhanced recovery programme following pancreaticoduodenectomy. HPB 2012;14:700-708.

43. Kennedy EP, Grenda TR, Sauter PK, Rosato EL, Chojnacki KA, Rosato FE Jr, Profeta BC, Doria C, Berger AC, Yeo CJ. Implementation of a critical pathway for distal pancreatectomy at an academic institution. $J$ Gastrointest Surg 2009;13:938-944.

44. di Sebastiano P, Festa L, De Bonis A, Ciuffreda A, Valvano MR, Andriulli A, di Mola FF. A modified fasttrack program for pancreatic surgery: a prospective single-center experience. Langenbecks Arch Surg 2011;396:345-351.

45. Berberat PO, Ingold H, Gulbinas A, Kleeff J, Müller MW, Gutt C, Weigand M, Friess H, Büchler MW. Fast track-different implications in pancreatic surgery. J Gastrointest Surg 2007;11:880-887.

46. Vlug MS, Wind J, Hollmann MW, Ubbink DT, Cense HA, Engel AF, Gerhards MF, van Wagensveld BA, van der Zaag ES, van Geloven AA, Sprangers MA, Cuesta MA, Bemelman WA; LAFA study group. Laparoscopy in combination with fast track multimodal management is the best perioperative strategy in patients undergoing colonic surgery: a randomized clinical trial (LAFA-study). Ann Surg 2011;254:868-875.

47. Gillissen F, Hoff C, Maessen JM, Winkens B, Teeuwen JH, von Meyenfeldt MF, Dejong CH. Structured synchronous implementation of an enhanced recovery program in elective colonic surgery in 33 hospitals in the Netherlands. World J Surg. 2013;37: 1082-1093.

48. Bassi C, Falconi M, Molinari E, Salvia R, Butturini G, Sartori N, Mantovani W, Pederzoli P. Reconstruction by pancreaticojejunostomy versus pancreaticogastrostomy following pancreatectomy: results of a comparative study. Ann Surg 2005;242:767-771.

49. Miedema BW, Sarr MG, van Heerden JA, Nagorney DM, Mcllrath DC, Ilstrup D. Complications following pancreaticoduodenectomy. Current management. Arch Surg 1992;127:945-949.

50. Büchler MW, Friess H, Wagner M, Kulli C, Wagener V, Z'Graggen K. Pancreatic fistula after pancreatic head resection. Br J Surg 2000;87:883-889.

51. Büchler MW, Wagner M, Schmied BM, Uhl W, Friess H, Z'graggen K. Changes in morbidity after pancreatic resection: toward the end of completion pancreatectomy. Arch Surg 2003;138:1310-1314.

52. Halloran CM, Ghaneh P, Bosonnet L, Hartley MN, Sutton R, Neoptolemos JP. Complications of pancreatic cancer resection. Dig Surg 2002;19:138-146.

53. Maessen J, Dejong CH, Hausel J, Nygren J, Lassen K, Andersen J, Kessels AG, Revhaug A, Kehlet H, Ljungqvist $\mathrm{O}$, Fearon KC, von Meyenfeldt MF. A protocol is not enough to implement an enhanced recovery programme for colorectal resection. Br J Surg 2007;94:224-231.

54. Ahmed J, Khan S, Gatt M, Kallam R, MacFie J. Compliance with enhanced recovery programmes in elective colorectal surgery. Br J Surg 2010;97:754-758.

55. van den Broek MA, van Dam RM, van Breukelen GJ, Bemelmans MH, Oussoultzoglou E, Pessaux $P$, Dejong $\mathrm{CH}$, Freemantle N, Olde Damink SW. Development of a composite endpoint for randomized controlled trials in liver surgery. Br J Surg 2011;98:1138-1145.

56. Lassen K, Coolsen MM, Slim K, Carli F, de Aguilar-Nascimento JE, Schäfer M, Parks RW, Fearon KC, Lobo DN, Demartines N, Braga M, Ljungqvist O, Dejong CH; ERAS ${ }^{\circledR}$ Society; European Society for Clinical Nutrition and Metabolism; International Association for Surgical Metabolism and Nutrition. Guidelines for perioperative care for pancreaticoduodenectomy: enhanced recovery after surgery (ERAS ${ }^{\circ}$ ) society recommendations. World J Surg. 2012;37:240-258. 


\title{
CHAPTER
}

\begin{abstract}
A systematic review of outcomes in patients
undergoing liver surgery in an enhanced recovery

after surgery pathway
\end{abstract}

MME Coolsen, EM Wong-Lun-Hing, RM van Dam, AA van der Wilt, K Slim, K Lassen, CHC Dejong

HPB (Oxford). 2013;15:245-251 


\section{ABSTRACT}

\section{Objectives}

Enhanced recovery after surgery (ERAS) or fast-track protocols have been implemented in different fields of surgery to attenuate the surgical stress response and accelerate recovery. The objective of this study was to systematically review the literature on outcomes of ERAS protocols applied in liver surgery.

\section{Methods}

The MEDLINE, EMBASE, PubMed and Cochrane Library databases were searched for randomized controlled trials (RCTs), comparative studies with historical controls and case series published between January 1966 and October 2011 comparing adult patients undergoing elective liver surgery in an ERAS program with those treated in a conventional manner. The primary outcome measure was hospital length of stay (LOS). Secondary outcome measures were time to functional recovery, and complication, readmission and mortality rates.

\section{Results}

A total of 307 articles were found, six of which were included in the review. These comprised two RCTs, three comparative studies with historical controls and one retrospective case series. Median LOS ranged from 4 days in an ERAS group to 11 days in a control group. Morbidity, mortality and readmission rates did not differ significantly between the groups. Only two studies assessed time to functional recovery. Functional recovery in these studies was reached 2 days before discharge.

\section{Conclusions}

This systematic review suggests that ERAS protocols can be successfully implemented in liver surgery. Length of stay is reduced without compromising morbidity, mortality or readmission rates. 


\section{INTRODUCTION}

Liver resection is the preferred treatment for a variety of primary and secondary liver tumors. Major abdominal surgical procedures such as hepatic resections cause a considerable surgical stress reaction and possible derangements in metabolic and pulmonary functions. Specific complications after hepatic resection include postoperative hemorrhage in the first hours to days after surgery, biliary leakage, intraabdominal abscess and liver failure in a later postoperative stage ${ }^{1}$. Improved operative techniques and insight into perioperative management have lowered mortality after liver resection to its current level of well below $5 \%$, but morbidity rates remain high and range between $30 \%$ and $50 \%{ }^{2-4}$.

In the past decade, multimodal enhanced recovery after surgery (ERAS) protocols or fast-track pathways have been applied in different forms of surgery. These pathways were developed to attenuate the surgical stress response and improve recovery, thereby decreasing postoperative complications and postoperative length of stay (LOS) in hospital ${ }^{5}$. ERAS protocols have gained territory quickly because of the associated cost efficiency derived from the reduction in LOS, an important issue in today's context of rapidly increasing health care costs and the consequent need for optimization. To date, studies that show ERAS protocols that reduce LOS and morbidity rates and improve patient satisfaction have been published in the contexts of vascular surgery ${ }^{6,7}$, musculoskeletal surgery, ${ }^{8}$ breast surgery ${ }^{9}$ and different forms of abdominal surgery ${ }^{10-12}$. Enhanced recovery after surgery protocols have also been implemented in liver surgery, but their effectiveness has not been studied extensively. The present systematic review was performed to evaluate the effects of ERAS protocols in liver surgery on time to recovery following surgery and postoperative hospital LOS, and to examine the effects of the implementation of such proto- cols on complication and readmission rates following liver surgery.

\section{MATERIALS AND METHODS}

\section{Search strategy}

A systematic search was performed in PubMed, the Cochrane Library, EMBASE and MEDLINE for studies published between January 1966 and October 2011. Languages were restricted to English, Dutch and German. The following search terms were applied using the Boolean operators 'AND' and 'OR': 'clinical pathway', 'critical pathway', 'enhanced recovery', 'accelerated', 'perioperative' and 'fast track', combined with 'liver', 'hepatic' and 'resection'. Synonyms of terms were also used in the search. The reference lists of selected papers were hand-searched for articles that were not 
retrieved in the database search. If necessary, authors of relevant articles were contacted to obtain additional information.

\section{Inclusion and exclusion criteria}

Studies were considered eligible for inclusion if they met all of the following inclusion criteria: (i) they reported on adult patients undergoing elective open or laparoscopic liver surgery; (ii) they described an enhanced recovery program with at least four different perioperative elements, and (iii) they reported outcomes including LOS, postoperative morbidity and mortality, and readmission rates. Studies were excluded if they: (i) described a single intervention in perioperative care rather than a group of interventions combined in an enhanced recovery program; (ii) reported on emergency, non-elective or transplantation surgery, and (iii) reported a non-systematic review. Table 3.1 lists a summary of ERAS items applicable to liver surgery. The items are supported by varying levels of evidence ${ }^{13}$. Perioperative care is considered to fall within an ERAS protocol when at least four different items are included, covering the pre-, intra- and postoperative periods ${ }^{14-15}$.

Table 3.1 Summary of elements in an enhanced recovery after surgery (ERAS) program applicable to liver surgery

\begin{tabular}{ll}
\hline Evidence-based factors & Probably useful factors \\
\hline $\begin{array}{l}\text { No oral bowel preparation } \\
\text { Preoperative feeding: carbohydrate loading up to } 2 \mathrm{~h}\end{array}$ & $\begin{array}{l}\text { Preoperative counseling } \\
\text { Provision of i.v. analgesia }\end{array}$ \\
$\begin{array}{l}\text { before surgery } \\
\text { No pre-anesthetic medication }\end{array}$ & $\begin{array}{l}\text { Stimulation of bowel movement with laxatives } \\
\text { Enti-thrombotic prophylaxis }\end{array}$ \\
$\begin{array}{l}\text { Single-dose antibiotics } \\
\text { Epidural analgesia }\end{array}$ & Audit \\
$\begin{array}{l}\text { Prevention of postoperative nausea and vomiting } \\
\text { Avoidance of hypothermia }\end{array}$ & \\
No routine drainage of peritoneal cavity & \\
No postoperative nasogastric intubation & \\
Good fluid balance & \\
Removal of urinary catheter on day 1 & \\
Normal food at will after surgery from day 1 &
\end{tabular}

Evidence-based factors: separate items are graded as being supported by level 1 or level 2 evidence (according to the guidelines of the Oxford Center for Evidence-Based Medicine ${ }_{13}$ ). Probably useful factors: evidence is less strong, but these factors are felt to be useful because the items are most probably qualityenhancing, are associated generally with a low incidence of adverse effects and low costs.

\section{Outcome measures}

The primary outcome measure of this systematic review was hospital LOS. Secondary outcome measures were time to functional recovery, complication rates, readmissions and mortality rates. Criteria for functional recovery were: good pain control with oral 
analgesia only; tolerance for solid food; no requirement for i.v. fluids; passage of stool, and independent mobility at the preoperative level ${ }^{16}$.

\section{Study selection and data selection}

Abstracts and titles of studies identified by the search were read by two authors (MMEC and AAvdW), each of whom independently made a first selection of studies. These first selections were compared and, in the event that the inclusion of a study required discussion, a third reviewer (RMvD) was consulted. Second and final selections were made independently by each of the two authors after reading the full-text articles. Both randomized as well as non-randomized studies were eligible for inclusion as long as they met the inclusion criteria. The methodological quality of the included studies was assessed using the MINORS (methodological index for non-randomized

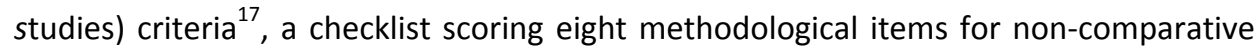
studies (maximum of 16 points) and an additional four items for comparative studies (maximum of 24 points). Missing data were obtained by contacting the authors of the relevant studies.

Data on the following factors were extracted from the included articles: postoperative LOS; number of patients included; patient ages; types of surgery; discharge criteria; functional recovery; mortality; morbidity; readmissions, and protocol adherence.

\section{Statistical analysis}

As the search strategy did not identify any randomized controlled trials (RCTs) evaluating the outcomes of ERAS protocols against those of traditional care, the MOOSE (meta-analysis of observational studies in epidemiology) checklist for metaanalysis of observational studies was used to assess the possibility of conducting a meta-analysis ${ }^{18}$. The included studies were considered to be too heterogeneous to support this and therefore no attempt at meta-analysis was made. Results are subsequently presented in tables and figures.

\section{RESULTS}

\section{Selected articles and characteristics of the studies}

The literature search produced 307 articles, of which 300 were excluded after their abstracts had been read in the first round of selection because they did not concern the evaluation of a fast- track program in liver surgery (Figure 3.1). After evaluation of the remaining seven papers, one was excluded because it was a non- systematic review ${ }^{19}$. 


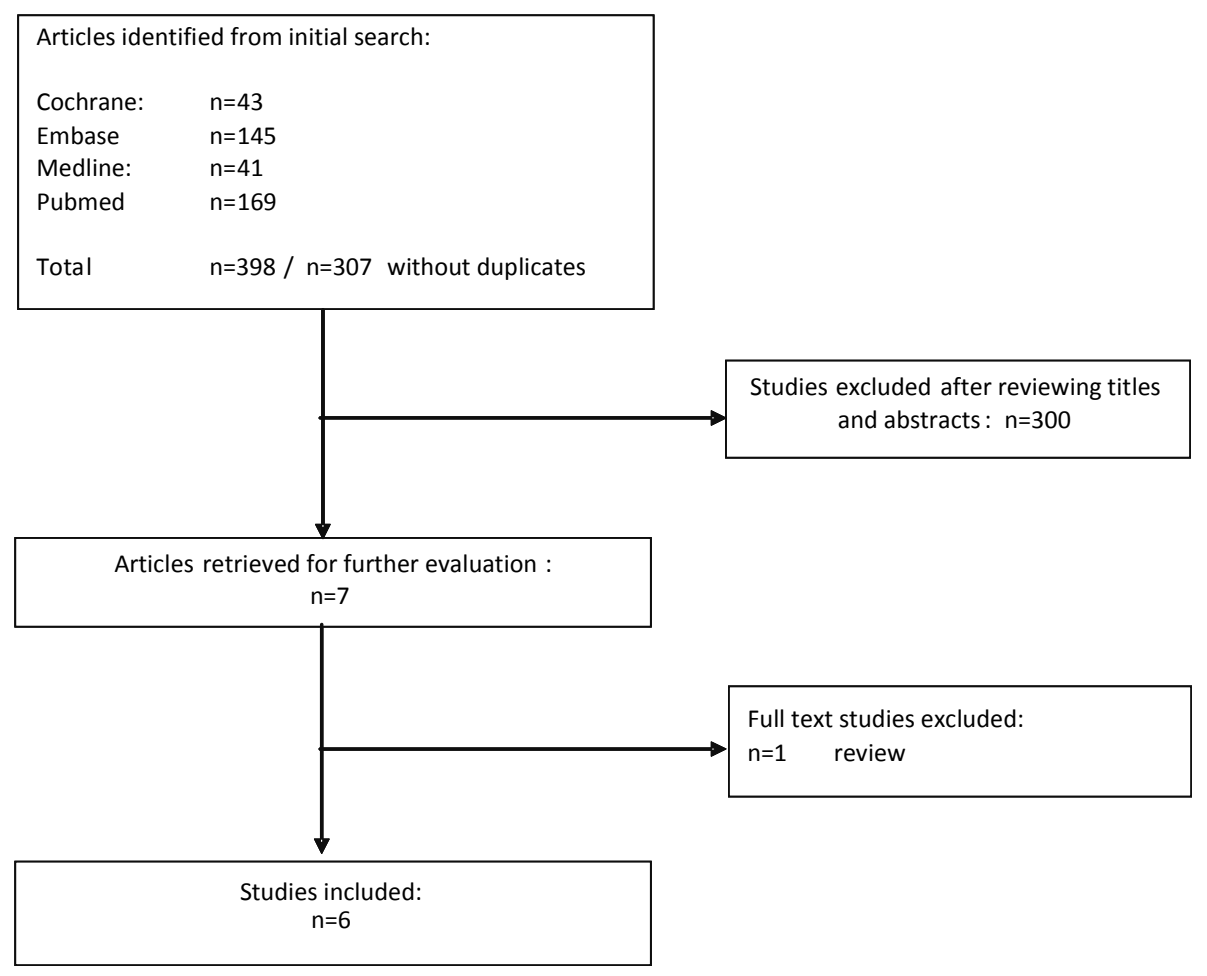

Figure 3.1 Study selection for systematic review

Finally, six papers were included in this systematic review. The details of the included studies are shown in Table 3.2. There were no reports of RCTs evaluating the outcomes of an ERAS program against those of traditional care. In two RCTs, both study groups were treated in an ERAS program. One of these RCTs evaluated the use of laxatives and oral nutritional supplements within an ERAS program ${ }^{20}$ and one assessed different forms of postoperative analgesia in two groups managed in a fast-track program ${ }^{21}$. Three comparative studies with historical controls and one retrospective case series were also included $^{16,22-24}$.

All studies included patients undergoing various forms of liver resection, including (extended) hemi-hepatectomy, metastasectomy, sectionectomy, central resection and repeat hepatectomy. One study did not include major hepatectomies; all patients in this study underwent laparoscopic liver resection ${ }^{20}$. All studies included a consecutive series of patients. Follow-up was 30 days in four studies and 3-6 months in one study. One study did not report the duration of follow-up. 
Table 3.2 Study characteristics and quality assessment

\begin{tabular}{|c|c|c|c|c|c|c|c|c|}
\hline \multirow[t]{2}{*}{ Study } & \multirow[t]{2}{*}{$\begin{array}{l}\text { Type of } \\
\text { surgery }\end{array}$} & \multirow[t]{2}{*}{ Study design } & \multirow{2}{*}{$\begin{array}{l}\text { No of } \\
\text { patients in } \\
\text { study/ } \\
\text { control } \\
\text { group, } \mathrm{n}\end{array}$} & \multirow{2}{*}{$\begin{array}{c}\text { Consecutive } \\
\text { series of } \\
\text { patients }\end{array}$} & \multirow{2}{*}{$\begin{array}{l}\text { Length of } \\
\text { follow- } \\
\text { up }\end{array}$} & \multicolumn{2}{|c|}{$\begin{array}{c}\text { Age, years, median } \\
\text { (range( }\end{array}$} & \multirow[t]{2}{*}{$\begin{array}{l}\text { MINORS } \\
\text { score }\end{array}$} \\
\hline & & & & & & ERAS group & $\begin{array}{l}\text { Control } \\
\text { group }\end{array}$ & \\
\hline $\begin{array}{l}\text { van Dam } \\
\text { et al. } 2008^{16}\end{array}$ & $\begin{array}{l}\text { HE, EHE, } \\
\text { ME, SE, } \\
\text { CR, RHE }\end{array}$ & $\begin{array}{l}\text { Comparative } \\
\text { study with } \\
\text { historical } \\
\text { controls }\end{array}$ & $61 / 100$ & Yes & 30 days & $62(24-82)$ & $60(20-81)$ & $18 / 24$ \\
\hline $\begin{array}{l}\text { Lin } \\
\text { et al. } 2000^{22}\end{array}$ & $\begin{array}{l}\text { SE, HE, } \\
\text { EHE, CR }\end{array}$ & $\begin{array}{l}\text { Comparative } \\
\text { study with } \\
\text { historical } \\
\text { controls }\end{array}$ & $56 / 61$ & Yes & 30 days & $57(23-73)$ & $55(22-81)$ & $17 / 24$ \\
\hline $\begin{array}{l}\text { Stoot } \\
\text { et al. } 2009^{23}\end{array}$ & $\begin{array}{l}\text { Laparo- } \\
\text { scopic: } \\
\text { ME, SE, } \\
\text { LLS }\end{array}$ & $\begin{array}{l}\text { Comparative } \\
\text { study with } \\
\text { historical } \\
\text { controls }\end{array}$ & $13 / 13$ & Yes & $\begin{array}{c}\text { 3-6 } \\
\text { months }\end{array}$ & $55(34-82)$ & $45(26-70)$ & $19 / 24$ \\
\hline $\begin{array}{l}\text { Hendry } \\
\text { et al. } 2010^{20}\end{array}$ & $\begin{array}{l}\mathrm{HE}, \mathrm{ME} \\
\mathrm{SE}, \mathrm{CR}\end{array}$ & $\mathrm{RCT}$ & $68^{a}$ & Yes & 30 days & $62(53-69)$ & - & $13 / 16$ \\
\hline $\begin{array}{l}\text { Koea } \\
\text { et al. } 2009^{21}\end{array}$ & $\begin{array}{l}\mathrm{HE}, \mathrm{EHE}, \\
\mathrm{ME}, \mathrm{SE}\end{array}$ & $\mathrm{RCT}$ & $100^{\mathrm{a}}$ & Yes & 30 days & $60(23-83)$ & - & $11 / 16$ \\
\hline $\begin{array}{l}\text { MacKay \& } \\
\text { O'Dwyer2008 }\end{array}$ & $\mathrm{HE}, \mathrm{SE}$ & $\begin{array}{c}\text { Retrospective } \\
\text { case series }\end{array}$ & 12 & Yes & Unknown & $60(43-74)$ & - & $9 / 16$ \\
\hline
\end{tabular}

${ }^{a}$ Patients in the control and experimental arms were all treated according to ERAS protocols. ERAS, enhanced recovery after surgery; $\mathrm{HE}$, hemi-hepatectomy; $\mathrm{EHE}$, extended hemi-hepatectomy; $\mathrm{ME}$, metastatectomy; $\mathrm{SE}$, segmentectomy; $\mathrm{CR}$, central resection; RHE, repeat hemo-hepatectomy; LLS, left lateral sectionectomy; RCT, randomized controlled trial

Age and other patient characteristics did not differ significantly among the patient groups described in the selected studies. Methodological quality assessed using the MINORS criteria was scored in the range of 17-19 points (of a maximum of 24 points) in comparative studies. Non-comparative studies achieved MINORS scores in the range of 8-13 points (of a maximum of 16 points).

Most studies described the enhanced recovery program in detail. A summary of the specific ERAS elements included in the different studies is shown in Table 3.3. Fourteen protocol elements were identified. Most studies included the majority of these elements; one study included only seven elements.

\section{Primary and secondary outcome measures}

Table 3.4 outlines postoperative outcomes after implementation of an ERAS program. Hospital LOS decreased significantly in the three comparative studies after ERAS implementation, in which median LOS was 5-7 days in the ERAS groups and 7-11 days in the traditional care groups. 


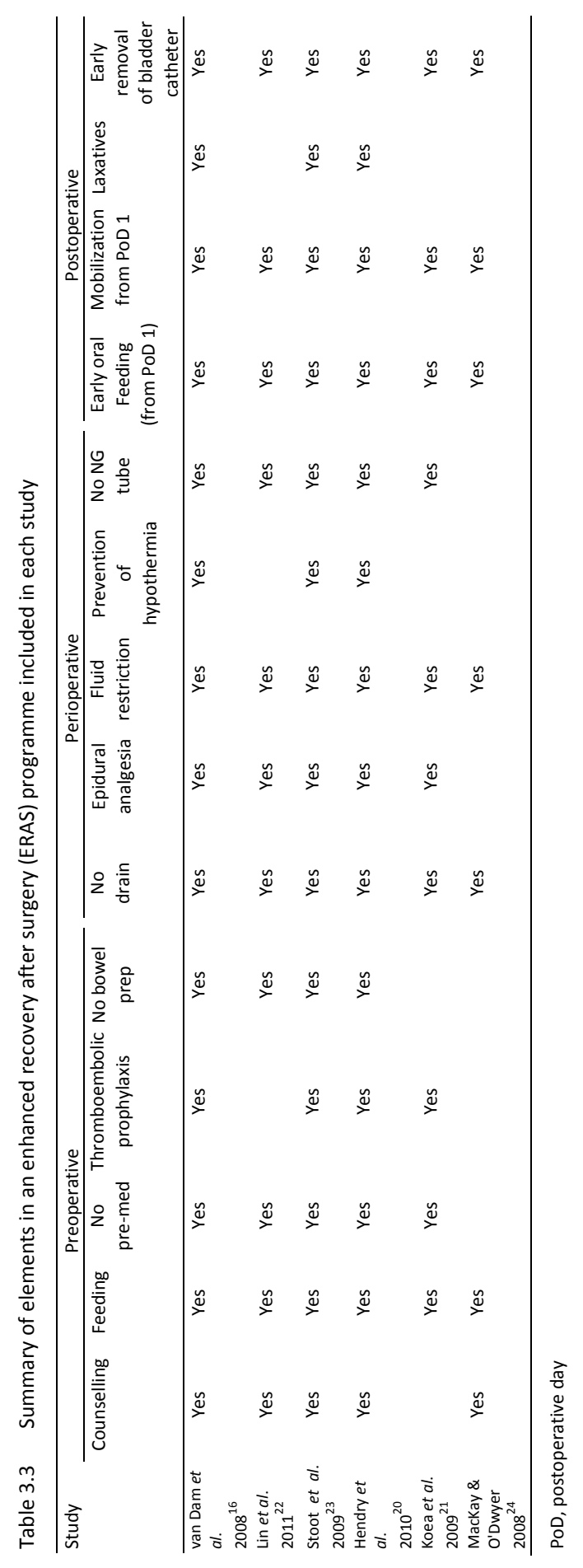



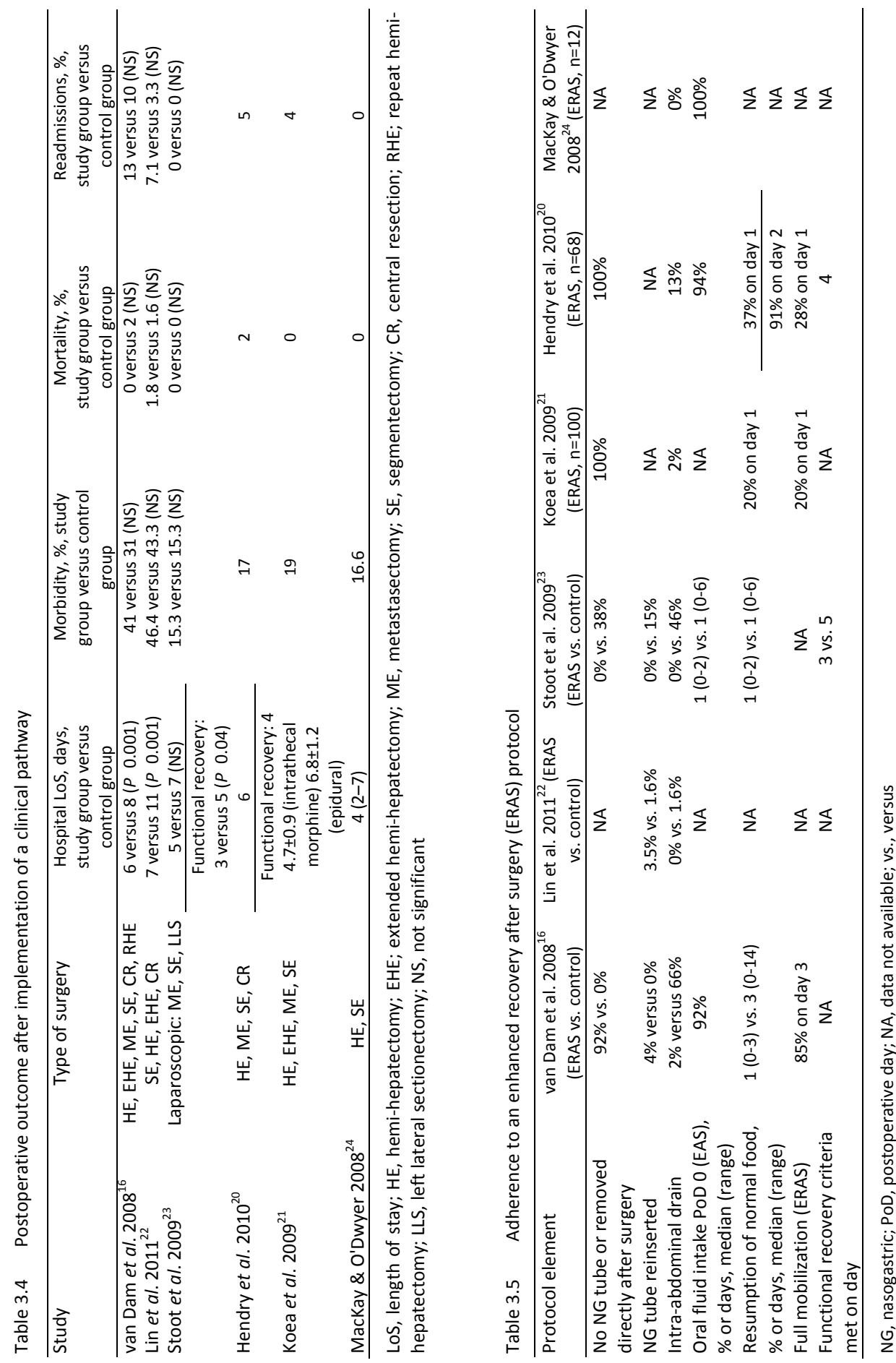
In the non-comparative studies, postoperative LOS after liver resection ranged between 4 days and 7 days. In the study by Stoot et al. ${ }^{21}$, all patients underwent laparoscopic liver resection. The median postoperative LOS was 5 days in the ERAS group and 7 days in the traditional care group; however, this difference was not statistically significant and the study did not include major liver resections. It is noteworthy that only two studies $^{17,20}$ assessed time to functional recovery and the reasons for delayed discharge. In both studies time to functional recovery was achieved 2 days prior to actual discharge from hospital. The main reasons for later discharge were concern for complications or extensive surgery, low patient confidence, and transport-related or other social problems.

Table 3.5 shows the extent of protocol adherence. The level of adherence to protocol was moderate in the studies included. Generally, nasogastric tubes were either not used or were immediately removed after surgery. The proportion of patients requiring the reinsertion of a nasogastric tube was low. In the study groups, intra-abdominal drains were used in only $2-13 \%$ of patients. The majority of patients resumed oral fluid intake on the day of surgery and achieved a normal diet on days 1 or 2 . The percentage of patients mobilized on the first postoperative day was low, with rates of $20-28 \%$ reported in only two studies ${ }^{20-21}$. In one study full mobilization was achieved on day 3 by $85 \%$ of patients ${ }^{16}$.

\section{DISCUSSION}

This systematic review examined the use of ERAS protocols in liver surgery in three comparative studies with historical controls, two RCTs and one case series. The results suggest that an enhanced recovery protocol can be successfully implemented in liver surgery. Hospital LOS was reduced and functional recovery was accelerated without compromising morbidity or mortality rates, and readmission rates were not significantly increased. The present results are in line with a recent review describing the use of fast-track protocols in hepato-pancreatic resections ${ }^{25}$.

At least four items in the pre-, peri- and postoperative periods must be included in an ERAS protocol for the protocol to be considered of value ${ }^{14,15}$. The studies in this review incorporated an average of 12 of 14 items (range: 7-14 items). In large series of patients undergoing liver surgery, LOS varies between 8 days and 14 days. ${ }^{3,4}$ All of the studies included in this review reported a shorter LOS in the ERAS study group. Two studies assessed time to functional recovery, which was significantly lower than total LOS. In many studies, LOS is reported as a primary outcome parameter. However, the use of this outcome may not always be appropriate as discharge is often delayed by a variety of other factors that may be unrelated to the true outcomes of the procedure ${ }^{26}$. The present authors therefore propose that time to functional recovery should be used as an outcome measure rather than LOS. 
Morbidity rates reported in the literature vary from $38 \%$ to $45 \%, 27$ and are comparable with the complication rates reported in the studies in the present review. However, it should be noted that complications in the studies included here were not always reported using a validated classification system (e.g. Dindo-Clavien or Accordion classification $^{28,29}$ ). This makes it more difficult to make meaningful comparisons of morbidity among the different centers.

The reporting of adherence to the various elements of the protocol was rather low in the included studies, especially as far as the introduction of normal diet and fluids was concerned. As Maessen et al. ${ }^{30}$ have observed, the reporting of adherence to protocol seems to be problematic in a considerable number of international studies. This impedes comparisons among studies ${ }^{30}$. The use of self-report patient diaries and continuous education of nurses and staff may represent strategies for overcoming this difficulty.

Overall, the methodological quality of the studies included in the present review, as assessed according to the MINORs criteria, was acceptable. However, this systematic review is limited by the fact that no RCTs comparing fast-track with standard care were available for inclusion (the RCTs included treated both the patient and control groups according to an ERAS protocol) and only case series and comparative studies using historical controls were included. The studies included were considered to be too heterogeneous to allow a meta-analysis. Another limitation of this review is that the individual studies used slightly different study protocols, with the result that the items incorporated in the various protocols are not identical and thus these studies are not fully comparable. However, a recent study by Ahmed et al. ${ }^{31}$ compared adherence to protocol in two groups of patients undergoing colorectal surgery and showed that outcome was unaltered in the study group in which adherence to some elements of the study protocol (e.g. preoperative carbohydrate loading and early fluid and diet introduction) was significantly lower. From this, it seems reasonable to conclude that not every item of an ERAS protocol makes an independent contribution to enhanced recovery, but, rather, it is the combination of different items in a structured care pathway that determines the outcome. This might also to some degree reflect a Hawthorne, or trial, effect, indicating a positive effect resulting from the implementation per se of a complex and comprehensive intervention.

Kehlet first introduced ERAS protocols in colon surgery in $1997^{32}$. Now, 15 years later, several items drawn from ERAS protocols are increasingly implemented in modern care worldwide. However, in many surgical fields, ERAS protocols have not yet been accepted as standard care. In the context of liver surgery, ERAS was first described in $2008^{24}$, since when only five studies examining an ERAS protocol in this field have been published $^{16,20-23}$ and three of these were performed by the same study group ${ }^{16,21,23}$. This seems to illustrate a limited international implementation of ERAS protocols in liver surgery. 
Although the methodology used in the studies included is not optimal, the results are consistent and seem to indicate clear advantages in terms of recovery. Although most centers today perform a proportion of resections laparoscopically, the present results serve to illustrate what can be achieved in open surgery and hence serve as a backdrop against which advances in technique and subsequent results can be compared.

In summary, this systematic review shows that it is feasible and safe to implement an ERAS protocol in hepatic surgery. The available evidence suggests that LOS is shortened without comprising morbidity, mortality or readmission rates. In view of the limited number of studies and the discrepancies in reporting among them, the present authors recommend the application of a standardized system of classifying complications, the accurate reporting of adherence to protocol, and the use of time to functional recovery as a primary outcome measure in future studies in order to enhance quality and comparability. 


\section{REFERENCES}

1. van den Broek MA, van Dam RM, van Breukelen GJ, Bemelmans MH, Oussoultzoglou E, Pessaux P, Dejong $\mathrm{CH}$, Freemantle N, Olde Damink SW.. Development of a composite endpoint for randomized controlled trials in liver surgery. Br J Surg 2011;98:1138-1145.

2. Belghiti J, Hiramatsu K, Benoist S, Massault P, Sauvanet A, Farges O. Seven hundred forty-seven hepatectomies in the 1990s: an update to evaluate the actual risk of liver resection. J Am Coll Surg 2000;191:38-46.

3. Dimick JB, Wainess RM, Cowan JA, Upchurch GR, Jr, Knol JA, Colletti LM. National trends in the use and outcomes of hepatic resection. J Am Coll Surg 2004;199:31-38.

4. Jarnagin WR, Gonen M, Fong Y, DeMatteo RP, Ben-Porat L, Little S, Corvera C, Weber S, Blumgart LH. Improvement in perioperative outcome after hepatic resection: analysis of 1803 consecutive cases over the past decade. Ann Surg 2002;236:397-407.

5. Kehlet H, Wilmore DW. Multimodal strategies to improve surgical outcome. Am J Surg 2002;183: 630-641.

6. Brustia P, Renghi A, Gramaglia L, Porta C, Cassatella R, De Angelis R, Tiboldo F. Mininvasive abdominal aortic surgery. Early recovery and reduced hospitalization after multidisciplinary approach. J Cardiovasc Surg (Torino) 2003;44:629-635.

7. Podore PC, Throop EB. Infrarenal aortic surgery with a 3-day hospital stay: a report on success with a clinical pathway. J Vasc Surg 1999;29:787-792.

8. Barbieri A, Vanhaecht K, Van Herck P, Sermeus W, Faggiano F, Marchisio S, Panella M. Effects of clinical pathways in the joint replacement: a meta-analysis. BMC Med 2009;7:32.

9. Arsalani-Zadeh R, ElFadl D, Yassin N, MacFie J. Evidence-based review of enhancing postoperative recovery after breast surgery. Br J Surg 2011;98:181-196.

10. Rawlinson A, Kang P, Evans J, Khanna A. A systematic review of enhanced recovery protocols in colorectal surgery. Ann R Coll Surg Engl 2011;93:583-588.

11. McCarty TM, Arnold DT, Lamont JP, Fisher TL, Kuhn JA. Optimizing outcomes in bariatric surgery: outpatient laparoscopic gastric bypass. Ann Surg 2005;242:494-498.

12. Kirsh EJ, Worwag EM, Sinner M, Chodak GW. Using outcome data and patient satisfaction surveys to develop policies regarding minimum length of hospitalization after radical prostatectomy. Urology 2000;56:101-106.

13. Phillips B, Ball C, Sackett D, Badenoch D, Straus S, Haynes B, et al. Levels of evidence and grades of recommendations. Oxford Centre for Evidence-Based Medicine. Available at http://www.cebm.net/ index.aspx?o=1025. 2011 (last accessed 19 September 2012)

14. Varadhan KK, Neal KR, Dejong $\mathrm{CH}$, Fearon KC, Ljungqvist O, Lobo DN. The enhanced recovery after surgery (ERAS) pathway for patients undergoing major elective open colorectal surgery: a meta-analysis of randomized controlled trials. Clin Nutr 2010;29:434-440.

15. Wind J, Polle SW, Fung Kon Jin PH, Dejong CH, von Meyenfeldt MF, Ubbink DT, Gouma DJ, Bemelman WA; Laparoscopy and/or Fast Track Multimodal Management Versus Standard Care (LAFA) Study Group; Enhanced Recovery after Surgery (ERAS) Group. Systematic review of enhanced recovery programmes in colonic surgery. Br J Surg 2006;93:800-809.

16. van Dam RM, Hendry PO, Coolsen MM, Bemelmans MH, Lassen K, Revhaug A, Fearon KC, Garden OJ, Dejong $\mathrm{CH}$; Enhanced Recovery After Surgery (ERAS) Group. Initial experience with a multimodal enhanced recovery programme in patients undergoing liver resection. Br J Surg 2008;95:969-975.

17. Slim K, Nini E, Forestier D, Kwiatkowski F, Panis Y, Chipponi J. Methodological index for non-randomized studies (MINORS): development and validation of a new instrument. ANZ J Surg 2000;73: 712-716.

18. Stroup DF, Berlin JA, Morton SC, Olkin I, Williamson GD, Rennie D, Moher D, Becker BJ, Sipe TA, Thacker SB. Meta-analysis of observational studies in epidemiology: a proposal for reporting. Meta-analysis of Observational Studies in Epidemi- ology (MOOSE) group. JAMA 2000;283:2008-2012.

19. van Gulik T. Open versus laparoscopic resection for liver tumours. HPB 2009;11:465-468. 
20. Hendry PO, van Dam RM, Bukkems SF, McKeown DW, Parks RW, Preston T, Dejong CH, Garden OJ, Fearon KC; Enhanced Recovery After Surgery (ERAS) Group. Randomized clinical trial of laxatives and oral nutritional supplements within an enhanced recovery after surgery protocol following liver resection. Br J Surg 2010;97:1198-1206.

21. Koea JB, Young Y, Gunn K. Fast-track liver resection: the effect of a comprehensive care package and analgesia with single-dose intrathecal morphine with gabapentin or continuous epidural analgesia. HPB Surg 2009:271986.

22. Lin DX, Li X, Ye QW, Lin F, Li LL, Zhang QY. Implementation of a fast-track clinical pathway decreases postoperative length of stay and hospital charges for liver resection. Cell Biochem Biophys 2011;61: 413-419.

23. Stoot JH, van Dam RM, Busch OR, van Hillegersberg R, De Boer M, Olde Damink SW, Bemelmans MH, Dejong $\mathrm{CH}$; Enhanced Recovery After Surgery (ERAS) Group. The effect of a multimodal fast-track programme on outcomes in laparoscopic liver surgery: a multicentre pilot study. HPB 2009;11:140-144.

24. MacKay G, O'Dwyer PJ. Early discharge following liver resection for colorectal metastases. Scott Med J 2008;53:22-24

25. Spelt L, Ansari D, Sturesson C, Tingstedt B, Andersson R. Fast- track programmes for hepatopancreatic resections: where do we stand? HPB 2011;13:833-838.

26. Maessen JM, Dejong CH, Kessels AG, von Meyenfeldt MF. Length of stay: an inappropriate readout of the success of enhanced recovery programmes. World J Surg 2008;32:971-975.

27. McColl RJ, You X, Ghali WA, Kaplan G, Myers R, Dixon E. Recent trends of hepatic resection in Canada: 1995-2004. J Gastrointest Surg 2008;12:1839-1846.

28. Clavien PA, Barkun J, de Oliveira ML, Vauthey JN, Dindo D, Schulick RD, de Santibañes E, Pekolj J, Slankamenac K, Bassi C, Graf R, Vonlanthen R, Padbury R, Cameron JL, Makuuchi M. The Clavien-Dindo classification of surgical complications: five-year experience. Ann Surg 2009;250:187-196.

29. Strasberg SM, Linehan DC, Hawkins WG. The accordion severity grading system of surgical complications. Ann Surg 2009;250:177-186.

30. Maessen J, Dejong CH, Hausel J, Nygren J, Lassen K, Andersen J, Kessels AG, Revhaug A, Kehlet $H$, Ljungqvist $\mathrm{O}$, Fearon $\mathrm{KC}$, von Meyenfeldt MF. A protocol is not enough to implement an enhanced recovery programme for colorectal resection. Br J Surg 2007;94:224-231.

31. Ahmed J, Khan S, Gatt M, Kallam R, MacFie J. Compliance with enhanced recovery programmes in elective colorectal surgery. Br J Surg 2010;97:754-758.

32. Kehlet H. Multimodal approach to control postoperative pathophysiology and rehabilitation. $\mathrm{Br} J$ Anaesth 1997;78:606-617. 


\section{CHAPTER 4}

To drain or not to drain: a cumulative meta-analysis of the use of routine abdominal drains after pancreatic resection

AA van der Wilt, MME Coolsen, IHJT de Hingh, GJ van der Wilt, H Groenewoud, CHC Dejong, RM van Dam

HPB (Oxford). 2013;15:337-344 


\section{ABSTRACT}

\section{Background}

To warrant the adoption or rejection of health care interventions in daily practice, it is important to establish the point at which the available evidence is considered sufficiently conclusive. This process must avoid bias resulting from multiple testing and take account of heterogeneity across studies. The present paper addresses the issue of whether the available evidence may be considered sufficiently conclusive to continue or discontinue the current practice of postoperative abdominal drainage after pancreatic resection.

\section{Methods}

A systematic review was conducted of randomized and non-randomized studies comparing outcomes after routine intra-abdominal drainage with those after no drainage after pancreatic resection. Studies were retrieved from the PubMed, Cochrane Central Trial Register and EMBASE databases and meta-analyzed cumulatively, adjusting for multiple testing and heterogeneity using the iterated logarithm method.

\section{Results}

Three reports, describing, respectively, one randomized and two non-randomized studies with a comparative design, met the inclusion criteria predefined for primary studies reporting on drain management and complications after pancreatic resection. These studies included 89, 179 and 226 patients, respectively. The absolute differences in rates of postoperative complications in these studies were $-6.4 \%,-9.5 \%$ and $-6.3 \%$, respectively, in favor of the no-drain groups. The cumulative risk difference in major complications, adjusted for multiple testing and heterogeneity, was $-7.8 \%$, with a $95 \%$ confidence interval of $-20.2 \%$ to $4.7 \%(P=0.214)$.

\section{Conclusions}

The routine use of abdominal drains after pancreatic resection may result in a higher risk for major complications, but the evidence is inconclusive. 


\section{INTRODUCTION}

For several decades, the routine use of postoperative abdominal drains has been standard practice in abdominal surgery. The main rationale for this practice is the prevention of fluid collections in the abdomen and the detection of postoperative bleeding or anastomotic leakage ${ }^{1}$. However, the routine use of postoperative drains in abdominal surgery can itself provoke complications. These include hemorrhage, inflammation, retrograde bacterial migration, drain occlusion or loss, pain, and loss of fluids and electrolytes ${ }^{2}$. All such complications may delay recovery and prolong hospital stay. The standard use of drains is also interfering with attempts to accelerate recovery through enhanced recovery after surgery (ERAS) programs $s^{3,4}$. Therefore, it is no longer self-evident that the benefits of the routine use of postoperative drains after abdominal surgery outweigh the associated risks.

Pancreatic resection may represent a special case in this respect because a postoperative leakage of the pancreaticojejunostomy is generally considered to pose an extra risk to the patient's recovery and health as a result of the autolytic properties of pancreatic juices ${ }^{5}$. However, as long ago as 1992, Jeekel questioned the routine use of postoperative drainage after pancreatic resection ${ }^{6}$. Since then, several randomized and non-randomized studies have addressed the subject.

As evidence on the safety and effectiveness of health care interventions accrues over time, a crucially important challenge is to decide when the evidence that has amassed on the benefit or harm of an intervention is clinically and statistically sufficient to warrant its adoption or rejection in clinical practice. For this purpose, the method of cumulative meta-analysis has been developed ${ }^{7,8}$. In the present study, this method was used to assess whether there is currently sufficient evidence to omit post- operative drainage after pancreatic resection without undue complications.

\section{MATERIALS AND METHODS}

\section{Literature search}

A search of the PubMed, EMBASE and Cochrane Central Trial Register databases was performed to identify studies on routine peripancreatic drainage after pancreaticoduodenectomy (PD). Search terms included 'drainage', 'drain*', 'suction', 'pancreatectomy', 'pancreatic resection', 'pancreaticoduodenectomy', 'pancreat*', 'postoperative complications', 'complication*', 'fistula' and 'abscess'. The full search strategy is shown in Appendix S1. 


\section{Study selection and data extraction}

Eligible studies were assessed on predefined inclusion criteria. In order to be considered as eligible, studies were required to: (i) report primary data; (ii) include a study population consisting of patients with suspected or histologically proven pancreatic or periampullary malignancy; (iii) include a population of patients undergoing pancreatic resection, including PD or distal resection, and (iv) compare routine peripancreatic postoperative drainage with no drainage. To make optimal use of the available evidence, randomized as well as non-randomized studies, conducted prospectively as well as retrospectively, were included taking into account any heterogeneity in the analysis.

Two reviewers independently assessed all titles and abstracts for inclusion. Disagreements were resolved by discussion. Full texts of studies eligible for inclusion were retrieved. The following data were extracted from the included studies: study design; inclusion and exclusion criteria; population size; baseline characteristics; duration of follow-up; statistical analysis; details of surgery and postoperative care, and outcome data. The methodological quality of studies was assessed using the MINORS (methodological index for non-randomized studies) score ${ }^{9}$. This score has been found to be a valid and reliable measure of the methodological quality of surgical trials and can be used for both randomized and non- randomized studies. It rates the appropriateness of enrolment of patients, endpoints and their mode of assessment, follow-up, control group, and statistical analysis, as well as comparability at baseline.

\section{Cumulative meta-analysis}

The primary outcome of this analysis was risk for major postoperative complications. Major complications were defined as postoperative complications severe enough to result in radiologic, endoscopic or surgical intervention. Secondary outcome parameters were mortality rate and hospital length of stay (LOS). When necessary, authors were contacted and asked to provide details on the definition of endpoints. Quantitative data were entered into a database using the statistical software package StatsDirect ${ }^{10}$. For each study included, a $2 \times 2$ table was constructed to show the number of events (major complications) and non-events in the treatment (drain) and control (no-drain) groups. Risk differences and associated cumulative Z-scores were calculated, both unadjusted and adjusted, using the iterated logarithm method described by $\mathrm{Hu}$ et al. ${ }^{11}$. A random-effects model was used, with total variance consisting of within- and between-study variance. To control for type I error, an adjustment factor (I) of 1.5 was used, as recommended by Hu et al. ${ }^{11}$. 


\section{RESULTS}

\section{Studies included and their characteristics}

The search produced 206 hits in PubMed (1969 to August 2011). The 21 and 55 publications retrieved from the Cochrane Trial Register and EMBASE, respectively, did not identify further eligible studies (Figure 4.1). Three studies including a total of 494 patients met the predefined selection criteria. ${ }^{12-14}$ Characteristics of these studies are summarized in Table 4.1.

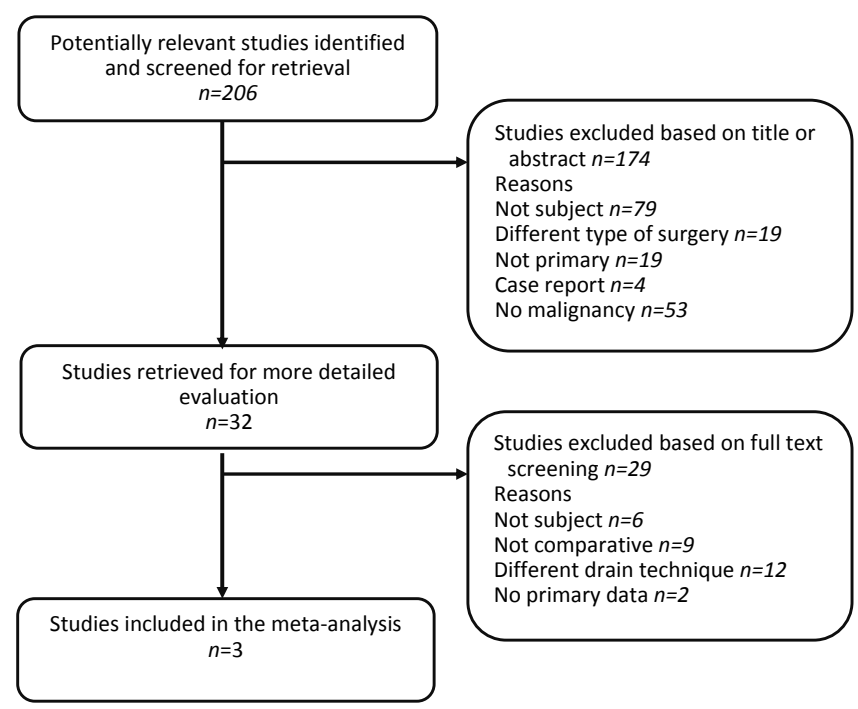

Figure 4.1 Flow-chart, showing number of studies retrieved from the literature databases and selection of studies on the basis of inclusion- and exclusion criteria

The study by Heslin et al. ${ }^{14}$ was a retrospective review of records of patients who underwent PD, with only little variation in surgery, including Roux-en-Y reconstruction and pylorus-preserving procedures. Patients in the drain group routinely received peripancreatic closed suction drains postoperatively. A total of 38 patients were not given drains; in 51 patients, closed suction drains were placed at the end of the procedure. Over $80 \%$ of the patients included had malignant disease. On histologic diagnosis, adenocarcinoma was found to be most prevalent. Locations of the tumor were the pancreas head (74\%), ampulla of Vater (13\%), duodenum (5\%) and distal common bile duct (10\%). Major complications were defined as death, reoperation, significant pancreatic leak, fascia dehiscence, re-intubation, pneumonia and intraabdominal abscess. Major complications occurred in $27 \%$ [95\% confidence interval (Cl) $16-42 \%]$ of the drain group and $21 \%$ (95\% Cl 10-37\%) of the no-drain group. Mean LOS was 12 days in both groups. Outcome measures are summarized in Table 4.2. 


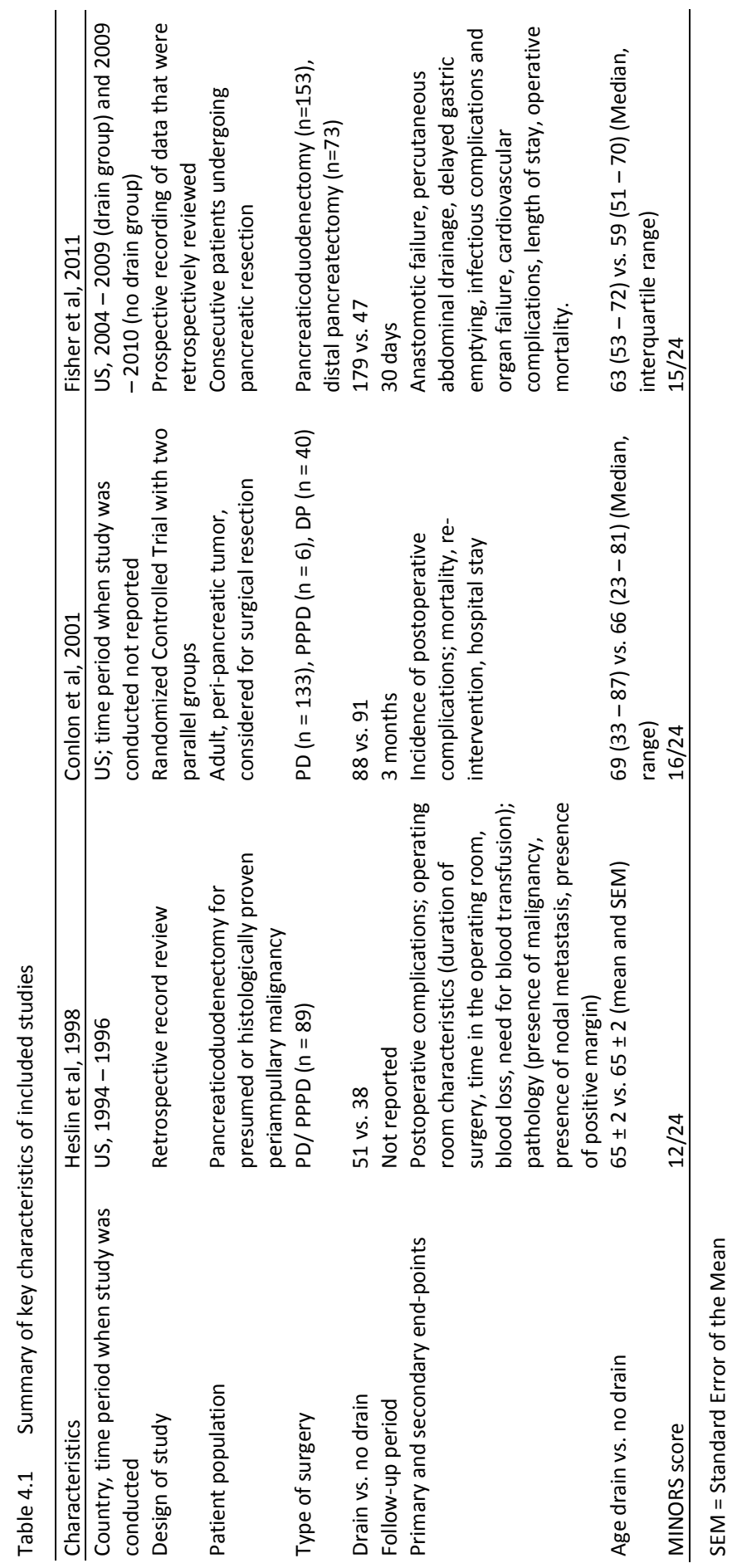




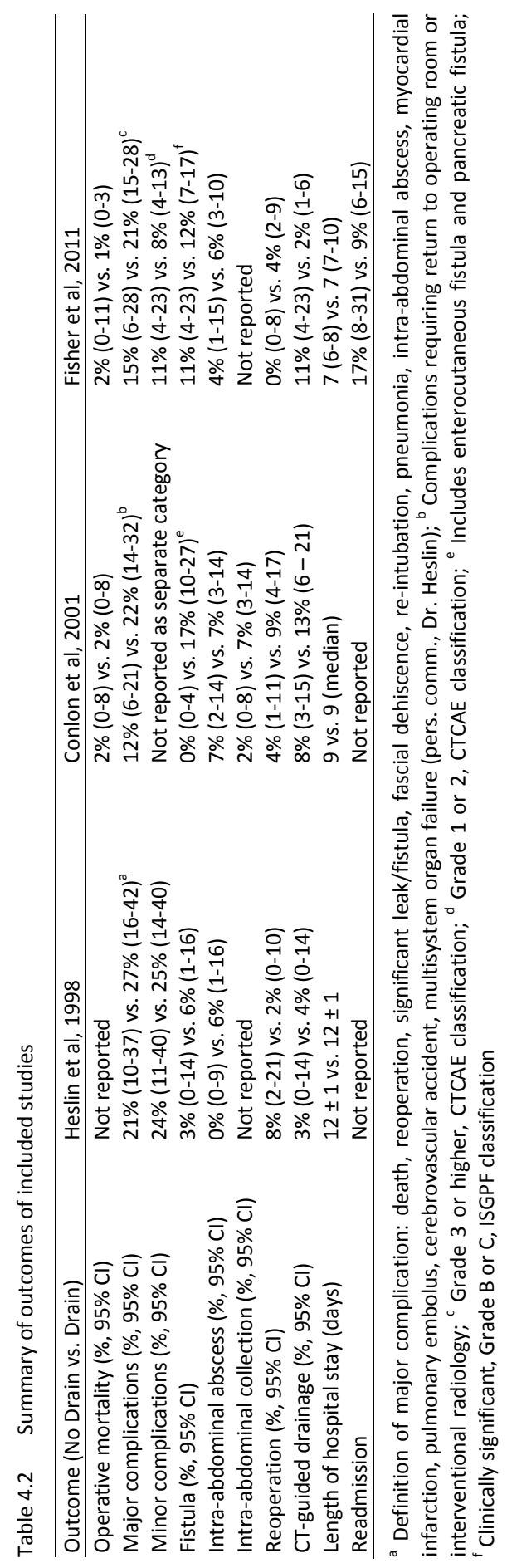


The study by Conlon et al. ${ }^{12}$ was a randomized controlled trial (RCT). It included a total of 179 patients with peripancreatic tumors who were considered for surgical treatment and randomized to receive no drain $(n=91)$ or a closed suction drain $(n=88)$ placed at the end of the procedure. A total of $78 \%$ of the procedures were PDs and $22 \%$ were distal pancreatectomies (DPs). Because the authors did not classify the severity of complications, complications requiring interventional radiologic procedures or a return to the operating room were rated as major complications. Such complications were reported in $22 \%$ (95\% Cl $14-32 \%)$ of patients in the drain group and $12 \%$ (95\% Cl 6-21\%) of those in the no-drain group. Mortality within 30 days after surgery was $2 \%$ in both groups. Hospital LOS did not differ between the two groups; overall median LOS was 9 days (range: 5-11 days). Twenty-nine patients were readmitted to hospital. Of these readmissions, 19 occurred in the drain group and 10 in the no-drain group $(P=0.07)$. The median duration of readmission was significantly longer in the patients in the drain group (10 days versus 5 days; $P<0.05$ )

Fisher et al. ${ }^{13}$ prospectively collected data for a total of 226 patients including 179 in whom postoperative drains were routinely placed after pancreatic resection (2004-2009) and 47 in whom no postoperative drains were placed after pancreatic resection (2009-2010). In this study, the Common Terminology Criteria for Adverse Events (CTCAE) index ${ }^{15}$ was used to record complications; events of grade 3 or greater were considered as major complications. Major complications occurred in $21 \%(95 \% \mathrm{Cl}$ $15-28 \%)$ of patients in the drain group and $15 \%(95 \% \mathrm{Cl} 6-28 \%)$ of patients without drains $(P=0.3)$. No differences in median hospital stay ( 7 days) were observed between the two cohorts. The readmission rate was significantly higher in the no-drain cohort (17\% versus $9 \% ; P=0.007)$. Of eight patients in the no-drain cohort who required readmission, five underwent percutaneous drainage for pancreatic fistula and the other three required observation only.

\section{Cumulative meta-analysis}

The results of the meta-analysis are presented in Table 4.3 and Figures 4.2 and 4.3. The proportions of patients with major postoperative complications were consistently lower in the no-drain groups com- pared with the drain groups. The differences in risk for major complications in the three studies ${ }^{12-14}$ were $-6.4 \%(95 \% \mathrm{Cl}-33.9 \%$ to $21.1 \%)$, $-9.5 \%(95 \% \mathrm{Cl}-24.2 \%$ to $6.9 \%)$ and $-6.3 \%(95 \% \mathrm{Cl}-20.2 \%$ to $4.7 \%)$, respectively. However, in none of the studies did this difference reach statistical significance. The unadjusted cumulative Z-score of the three studies ${ }^{12-14}$ was -2.13 , just crossing the critical value of -1.96 , which suggests that statistical significance was reached when the data from the study by Fisher et al. ${ }^{13}$ were combined with the data from the previous two studies ${ }^{12-14}$. However, after adjustment for multiple testing and heterogeneity using the iterated logarithm method, the cumulative Z-score was -1.24 , indicating that the difference had not reached statistical significance. The adjusted cumulative difference in risk for major complications for the three studies ${ }^{12-14}$ was $-7.8 \%$, with a 
$95 \% \mathrm{Cl}$ of $-20.2 \%$ (favoring no drainage) to $4.7 \%$ (favoring drainage).

Meta-analysis of the secondary endpoints (mortality and LOS) proved to be impossible because the requisite data on variance could not be extracted from the original studies $^{12-14}$ nor obtained from the authors.

Table 4.3 Difference in risk of major postoperative complications, in patients who had not routinely placed postoperative drains after pancreatic resection, as compared to patients who had such drains placed routinely. Individual Z-statistics and cumulative Z-statistics, unadjusted and adjusted for multiple testing and heterogeneity across studies

\begin{tabular}{lcccc}
\hline Study & $\begin{array}{c}\text { Risk } \\
\text { difference }\end{array}$ & $\begin{array}{c}\text { Z-statistic for each } \\
\text { individual study }\end{array}$ & $\begin{array}{c}\text { Cumulative } \\
\text { Z-statistic, unadjusted }\end{array}$ & $\begin{array}{c}\text { Cumulative Z-statistic } \\
\text { adjusted (LIL-based) } \\
\text { (Random effect model) }\end{array}$ \\
\hline Heslin 1998 & -0.064 & -0.703 & -0.703 & -0.653 \\
Conlon 2001 & -0.095 & -1.709 & -1.889 & -1.180 \\
Fisher 2011 & -0.063 & -1.051 & -2.128 & -1.240 \\
\hline
\end{tabular}

LIL = Law of Iterated Logarithm

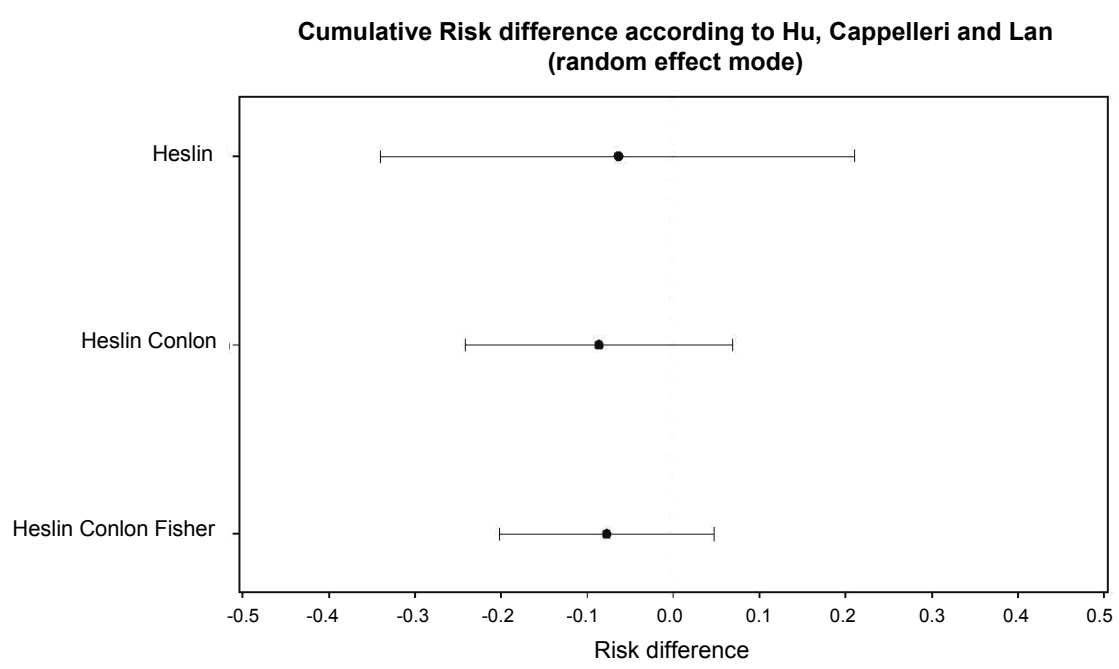

Figure 4.2 Cumulative random effects meta-analysis using LIL of 3 studies of routine postoperative drainage after pancreatic resection. Risk differences and 95\% confidence intervals. LIL = Law of Iterated Logarithm 
Cumulative Z-score according to Hu, Cappelleri and Lan

(random effect mode)

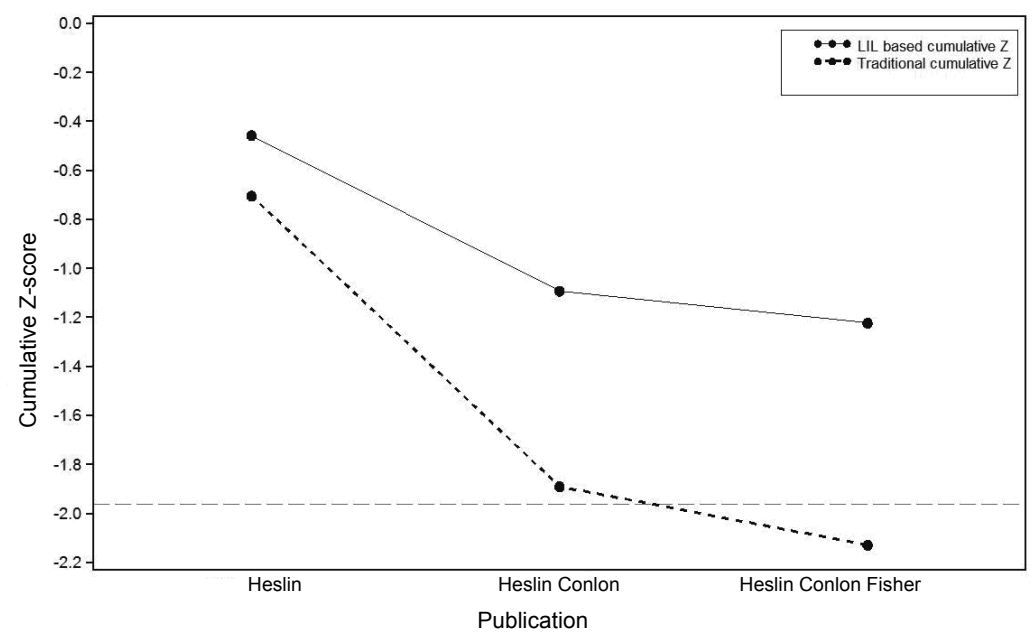

Figure 4.3 Cumulative meta-analysis of routine postoperative drainage after pancreatic resection. Cumulative Z-statistic for effect of omitting drainage on major postoperative complications. Traditional cumulative Z-statistic and Z-statistic adjusted for multiple testing and heterogeneity. LIL = Law of Iterated Logarithm

\section{DISCUSSION}

There is a persuasive logic to the practice of routine drainage after pancreatic surgery because drainage is assumed to allow for the early identification and monitoring of fluid collections and subsequently to prevent their negative impact on the patient's recovery. It seems, however, that the available evidence does not support this practice. In this study, a traditional meta-analysis of the avail- able evidence pointed to a significantly lower complication rate in the group without routine drainage. However, when a cumulative meta-analysis was performed with adjustment for repeated testing and heterogeneity, this difference was no longer statistically significant.

It is important to establish when the available evidence may be considered sufficiently conclusive to warrant the use of a particular intervention in daily practice. In this process, care should be taken to avoid bias resulting from multiple testing and heterogeneity across studies should be taken into account. Several methods have been developed to address this issue. Pogue and Yusuf ${ }^{16}$ pro- posed the alpha-spending approach for controlling type I error in cumulative meta-analysis. However, this approach is a closed sequential process, requiring the pre-specification of the final sample size, which is usually unknown ${ }^{11}$. The approach used in this study was recently developed by Hu et al. ${ }^{11}$. 
The method proposed by Hu et al. ${ }^{11}$ accounts for multiple testing and for heterogeneity across studies in a cumulative meta- analysis of trials with a binary endpoint. This method typically involves the performance of an updated meta-analysis every time that new evidence becomes available. Two challenges are encountered when using this method. Firstly, the procedure suffers from the problem of repeated testing and the associated inflated overall type I error (the unjustified acceptance of evidence of benefit or harm $)^{17}$. Secondly, because data may be pooled from studies that are spread over a prolonged period of time, medical technology, supportive care and patient populations may differ across studies, introducing substantial heterogeneity. The method, based on the law of Z-statistics, is an open and therefore more flexible approach towards controlling for type I error compared with those mentioned earlier. Moreover, it allows for reliable estimation of the between-study variance component, which is challenging, especially in a context in which the number of studies is small ${ }^{11}$.

In the studies ${ }^{12-14}$ included in the present analysis, substantial heterogeneity was found. This related to research design, definitions of endpoints, patient populations, types of interventions and supportive care. In particular, the definition of what constitutes a major complication differed among the studies, none of which used a standardized scale such as the Dindo-Clavien system of classification. An attempt was made to pool the different results as adequately as possible by comparing the different scoring systems. Such heterogeneity is likely to represent the rule rather than the exception, and therefore should be taken into account in any analysis in which studies are combined to make optimal use of the available evidence.

With respect to postoperative drains, several studies on other types of surgery, such as colon and liver surgery, have shown that the use of abdominal drains is not beneficial in terms of postoperative outcome and may even be harmful ${ }^{18}$. Pancreaticoduodenectomy is technically one of the most challenging surgical procedures and is associated with potential anastomotic break- down attributable to the activity of pancreatic enzymes; probably in response to this, surgeons tend to favor the conventional placement of abdominal drains. In order to avoid the risk for retrograde infection but to preserve the diagnostic safety drains are alleged to provide, some surgeons choose to place drains after every pancreatic resection and remove them after a short period of time. Kawai et al. ${ }^{19}$ assessed 104 consecutive patients undergoing pancreatic head resection and came to the conclusion that removing the drain on postoperative day 4 rather than day 8 reduced the incidence of complications. A soft pancreas is thought to result in a higher incidence of complications and therefore Kawai et al. ${ }^{19}$ also evaluated the impact of this clinical variable; however, the difference between groups in postoperative complication rates was not statistically significant. Bassi et al. ${ }^{20}$ assessed 114 patients with a low prior probability of pancreatic fistula, including only patients with drain amylase levels of $\leq 5000 \mathrm{U} / \mathrm{I}$. They concluded that the drain could safely be removed on postoperative day 3. Moreover, delayed removal of the drains (after postoperative day 5 ) led to a higher complication rate and increased 
hospital LOS and costs. Therefore, if abdominal drains are placed, it seems that their removal within a short period of time is justified.

It is important to note that two ${ }^{12,14}$ of the three studies ${ }^{12,14}$ included in this review included patients undergoing DP as well as those scheduled for PD among their study samples (113 of 494 patients, 22\%). Heslin et al. ${ }^{14}$ did not report the exact numbers of patients undergoing each type of resection. In the study by Conlon et al. ${ }^{12}$ patients in the DP group were more likely to be allocated to the no-drain group than those in the PD group (DP group: drain group, $n=15$ versus no-drain group, $n=25$; PD group, drain group, $n=73$ versus no-drain group, $n=66$ ). In the study by Fisher et al. ${ }^{13}$, both PD and DP patients were equally divided between the drain and no-drain groups. Nonetheless, this potentially increases complication rates and makes adequate comparison more difficult because leak rates after DP have been reported to be $22 \%$, whereas leak rates after PD have been reported to be $11 \%^{21,22}$.

The reluctance of surgeons to abandon abdominal drainage is understandable given the well-known detrimental effects of leakage of the pancreatojejunostomy and the absence of sufficiently powered, high-level evidence on prophylactic drainage after pancreatic resection. However, the development of less invasive radiologic intervention techniques benefits the treatment of post- operative complications such as intraabdominal abscesses ${ }^{23}$. This may contribute to a reduction in surgeon anxiety in relation to the development of severe complications and eventually to the willing- ness to abandon abdominal drainage after pancreatic resection.

A recently published overview of the available evidence for the use of abdominal drains after pancreatic resection adopted a slightly different approach, using a standard Mantel-Haenszel random-effects model and separate evaluations of overall and specific complications, respectively ${ }^{24}$. It was concluded that more evidence is required before any firm conclusions on the use of abdominal drains can be reached, which is in line with the present findings. This overview ${ }^{24}$ also included studies comparing the early and late removal of abdominal drains, which resulted in the inclusion of a higher number of studies. However, the study by Fisher et al. ${ }^{13}$ had not been published at the time this earlier analysis ${ }^{24}$ was conducted and as a consequence its results were not included in the analysis. Moreover, Diener et al. ${ }^{24}$ did not correct for heterogeneity and multiple testing and thus their results should be interpreted with great caution. Unfortunately, studies suitable for meta- analysis remain scarce and this affects the results of the present study to a similar extent as it has previous meta-analyses. Moreover, two of the studies ${ }^{12,14}$ included in the present analysis were conducted at the same center in different timeframes, which may have introduced bias to the present study. Nonetheless, both of these studies (randomized and non-randomized) were included in order to facilitate the pooling of data for as many patients as possible. In addition, in the current analysis, MINORS scores were used to rate the studies for quality. These scores provided evidence that the non-randomized studies included in the present analysis were of sufficient quality. It is of interest that the results of the 
non-randomized studies do not differ substantially from those of the RCT, which suggests that non-randomization did not introduce significant bias in the case of these two studies.

In conclusion, the current study shows the importance of taking into account multiple testing and heterogeneity within and between studies when conducting a metaanalysis. Without adjustment for the latter, the currently available evidence might be taken to imply that omitting drainage after pancreatic surgery leads to a statistically significant decrease in the risk for major complications. However, that conclusion is premature, as can be inferred from the present results when multiple testing and heterogeneity are taken into account. At present, an international, multicenter RCT intended to establish whether or not the prophylactic use of abdominal drains after PD can be abandoned is in preparation in the Netherlands. Until the results of such a trial become available, drain policy will depend on the surgeon's judgment. The available evidence suggests that it is reasonable to adopt a no-drain policy because not using a drain does not seem to increase postoperative complications. 


\section{REFERENCES}

1. Giovinazzo F, Butturini G, Salvia R, Mascetta G, Monsellato D, Marchegiani G, Pederzoli P, Bassi C. Drain management after pancreatic resection: state of the art. J Hepatobiliary Pancreat Sci 2011;18:779-784.

2. Dougherty SH, Simmons RL. The biology and practice of surgical drains. Part 1. Curr Probl Surg 1992; 29:559-623.

3. Berberat PO1, Ingold H, Gulbinas A, Kleeff J, Müller MW, Gutt C, Weigand M, Friess H, Büchler MW. Fast track - different implications in pancreatic surgery. J Gastrointest Surg 2007;11:880-887.

4. Kehlet H, Wilmore DW. Multimodal strategies to improve surgical outcome. Am J Surg 2002;183: 630-641.

5. Kleespies A, Albertsmeier M, Obeidat F, Seeliger H, Jauch KW, Bruns CJ. The challenge of pancreatic anastomosis. Langenbecks Arch Surg 2008;393:459-471.

6. Jeekel J. No abdominal drainage after Whipple's procedure. Br J Surg 1992;79:182.

7. Antman EM, Lau J, Kupelnick B, Mosteller F, Chalmers TC. A comparison of results of meta-analyses of randomized control trials and recommendations of clinical experts. Treatments for myocardial infarction. JAMA 1992;268:240-248.

8. Lau J, Antman EM, Jimenez-Silva J, Kupelnick B, Mosteller F, Chalmers TC. Cumulative meta-analysis of therapeutic trials for myocardial infarction. N Engl J Med 1992;327:248-254.

9. Slim K, Nini E, Forestier D, Kwiatkowski F, Panis Y, Chipponi J. Methodological index for non-randomized studies (MINORS): development and validation of a new instrument. ANZ J Surg 2003;73: 712-716.

10. Freemantle N. StatsDirect - statistical software for medical research in the 21st century. BMJ 2000; 321:1536.

11. Hu M, Cappelleri JC, Lan KK. Applying the law of iterated loga- rithm to control type I error in cumulative meta-analysis of binary outcomes. Clin Trials 2007;4:329-340.

12. Conlon KC, Labow D, Leung D, Smith A, Jarnagin W, Coit DG, Merchant N, Brennan MF. Prospective randomized clinical trial of the value of intraperitoneal drainage after pancreatic resection. Ann Surg 2001;234:487-493.

13. Fisher WE, Hodges SE, Silberfein EJ, Artinyan A, Ahern CH, Jo E, Brunicardi FC. Pancreatic resection without routine intraperitoneal drainage. HPB 2011;13:503-510.

14. Heslin MJ, Harrison LE, Brooks AD, Hochwald SN, Coit DG, Brennan MF. Is intra-abdominal drainage necessary after pancreaticoduo- denectomy? J Gastrointest Surg 1998;2:373-378.

15. Trotti A, Colevas AD, Setser A, Rusch V, Jaques D, Budach V, Langer C, Murphy B, Cumberlin R, Coleman CN, Rubin P. CTCAE v3.0: development of a comprehensive grading system for the adverse effects of cancer treatment. Semin Radiat Oncol 2003; 13:176-181.

16. Pogue JM, Yusuf S. Cumulating evidence from randomized trials: utilizing sequential monitoring boundaries for cumulative meta-analysis. Control Clin Trials 1997;18:580-593.

17. Berkey CS, Mosteller F, Lau J, Antman EM. Uncertainty of the time of first significance in random effects cumulative meta-analysis. Control Clin Trials 1996;17:357-371.

18. Petrowsky H, Demartines N, Rousson V, Clavien PA. Evidence- based value of prophylactic drainage in gastrointestinal surgery: a systematic review and meta-analyses. Ann Surg 2004;240:1074-1084.

19. Kawai M, Tani M, Terasawa H, Ina S, Hirono S, Nishioka R, et al. Early removal of prophylactic drains reduces the risk of intra-abdominal infections in patients with pancreatic head resection: prospective study for 104 consecutive patients. Ann Surg 2006;244:1-7.

20. Bassi C, Molinari E, Malleo G, Crippa S, Butturini G, Salvia R, et al. Early versus late drain removal after standard pancreatic resections: results of a prospective randomized trial. Ann Surg 2010;252:207-214.

21. Haga $Y$, Wada $Y$, Takeuchi H, Ikejiri K, Ikenaga M. Prediction of anastomotic leak and its prognosis in digestive surgery. World J Surg 2011;35:716-722.

22. Frozanpor F, Lundell L, Segersvard R, Arnelo U. The effect of prophylactic transpapillary pancreatic stent insertion on clinically significant leak rate following distal pancreatectomy: results of a prospective controlled clinical trial. Ann Surg 2012;255:1032-1036.

23. Iwata N, Kodera Y, Eguchi T, Ohashi N, Nakayama G, Koike M, Fujiwara M, Nakao A. Amylase concentration of the drainage fluid as a risk factor for intra-abdominal abscess following gastrectomy for gastric cancer. World J Surg 2010;34:1534-1539. 
24. Diener MK, Tadjalli-Mehr K, Wente MN, Kieser M, Buchler MW, Seiler CM. Risk-benefit assessment of closed intra-abdominal drains after pancreatic surgery: a systematic review and meta-analysis assessing the current state of evidence. Langenbecks Arch Surg 2011;396:41-52. 


\section{CHAPTER}

Development of a composite endpoint for

randomized controlled trials in pancreaticoduodenectomy

MME Coolsen, SHEM Clermonts, RM van Dam, B Winkens, M Malagó, GK Fusai, CHC Dejong, SWM Olde Damink

World J Surg 2014:38:1468-1475 


\section{ABSTRACT}

\section{Background}

Few randomized controlled trials (RCTs) have been performed in patients undergoing pancreaticoduodenectomy (PD). An important factor contributing to this is the large number of patients needed to adequately power RCTs for relevant clinical single endpoints. A PD-specific composite endpoint (CEP) could solve this problem. The aim of the present study was to develop a PD-specific CEP, consisting of complications related to PD, allowing reduction in sample sizes and improving the ability to compare outcomes.

\section{Methods}

PD-specific CEP components were selected after a systematic review of the literature and consensus between 25 international pancreatic surgeons. Ultimately, prospective cohorts of patients who underwent PD in two high-volume HPB centers (London, UK, and Maastricht, NL) were used to assess the event rate and effect of implementing a PD-specific CEP.

\section{Results}

From a total of 18 single-component endpoints, intra-abdominal abscess, sepsis, post-PD hemorrhage, bile leakage, gastrojejunostomy leakage, leakage of the pancreatic anastomosis, delayed gastric emptying, and operative mortality within 90 days were selected to be included the PD-specific CEP. All eight components had consensus definitions and a Dindo-Clavien classification of 3 or more. The incidence of the PD-specific CEP was $24.7 \%$ in the Maastricht cohort and $23.3 \%$ in the London cohort. These incidence rates led to a twofold reduction in the theoretical calculated sample size for an adequately powered RCT on PD using this CEP as a primary endpoint.

\section{Conclusion}

The proposed PD-specific CEP enables clinical investigators to adequately power RCTs on PD and increases the feasibility, comparability, and utility in meta-analysis. 


\section{INTRODUCTION}

In modern medicine, clinicians are obliged to practice evidence-based medicine. Metaanalyses and randomized controlled trials (RCTs) deliver the highest level of evidence regarding current practice. Conclusions of meta-analyses and large RCTs are incorporated in the latest guidelines and help to optimize care and outcome in surgical practice. In the field of pancreatic surgery, however, very few RCTs have been performed $^{1,2}$. Pancreaticoduodenectomy (PD) is the standard surgical procedure for patients with malignant and benign diseases of the pancreatic head and the periampullary region. With improved surgical techniques and perioperative management methods, PD has become increasingly safe. Mortality rates are relatively low $\left(<5 \%\right.$ in large series), and morbidity is in the range of $35-50 \%^{3,4}$. Centralization of PDs in high-volume centers has also contributed to improved outcomes ${ }^{5}$. Nonetheless PD remains one of the most complex operations in gastrointestinal surgery. Complications, such as anastomotic leakage (in particular of the hepatojejunostomy and pancreaticojejunostomy) and the formation of pancreatic fistula, have serious consequences for the patient. Clinical outcomes, such as surgery-related mortality or specific PD-related morbidity, are important for evaluation of the efficacy of surgical interventions. However, in RCTs focusing on pancreatic surgery the usefulness of these outcomes as a primary endpoint is limited because they are dichotomous and individually have a relatively low incidence. Consequently, large sample sizes are needed to obtain adequate statistical power. The aforementioned reasons could account for the low number of RCTs in this field ${ }^{2,6}$.

The use of a composite endpoint (CEP) that is specifically designed for PD could be a solution to the problem of the lack of RCTs in PD. In a CEP several individual, singleoutcome parameters are combined into one CEP; every time an individual outcome parameter is positive, the CEP is positive, but if in a patient more individual outcome parameters are positive, the CEP is only positive once. The individual parameters should be dichotomous, highly specific for the given surgical procedure, and of clinical relevance ${ }^{7,8}$. As the CEP combines individual outcome parameters (i.e. "individual, single endpoints") into one CEP, scoring a CEP results in a higher incidence than the incidence of individual specific endpoints. Using CEP as a primary endpoint therefore increases statistical power and allows a lower sample size when performing RCTs. It must be noted that a CEP is dichotomous (it occurs or it does not occur) and can therefore only be scored once in a patient, even though a patient has more than one complication that is included in the CEP. Therefore, a CEP will not result in underpowering. CEPs are already widely used in cardiovascular and pulmonary research and have recently been developed for liver surgery ${ }^{8-13}$. In pancreatic surgery, however, CEPs have been applied in only a few trials ${ }^{14-16}$. Furthermore, the CEPs used in the studies on pancreatic surgery all included different components, making the results incomparable. A CEP can be viewed as a minimal set of endpoints, a core outcome set (COS), that 
facilitates the conduct of future RCTs and meta-analyses and reduces the missing outcome parameters. The aim of the present study was to develop a standardized CEP specific for PD that can be used as a primary endpoint for future RCTs.

\section{METHODS}

The PD-specific CEP was developed according to a three-step method. First a systematic review of the literature was performed to identify all single-component clinical endpoints used in previous PD-related RCTs (development step 1). The systematic review was performed following the PRISMA statement. Second, a list of candidate components was defined and a web-based survey was used that allowed an international group of clinical experts in pancreatic survey to select the most relevant components for a CEP (development step 2). Consensus was reached between these experts and the study group to form a definitive CEP for PD. Third, validation of the CEP was conducted by assessing the event rate of the proposed CEP in two PD-patient cohorts at Maastricht University Medical Center (Maastricht, the Netherlands) and in the Royal Free Hospital London (London, United Kingdom). Both are tertiary referral centers for hepatopancreaticobiliary (HPB) surgery. Finally, the theoretical sample size was calculated of an RCT using the PD-specific CEP as a primary endpoint (development step 3).

Development step 1: identification of all single-component clinical endpoints in randomized trials on pancreaticoduodenectomy

\section{Search strategy}

Two researchers (M.C., S.C.) performed a review of the literature to identify all singlecomponent clinical outcomes used as a primary or secondary endpoint in trials on PD. The databases of PubMed, Embase, and the Cochrane Library were searched for the following search terms: "pancreatic resection" OR "Whipple" OR "distal pancreatectomy" OR "PD" combined with "RCT." Only English language articles published between January 2006 and August 2011 were included for further investigation.

\section{Eligibility criteria}

The following RCT eligibility criteria were used: (1) studies reporting on outcome after pancreatic surgery, (2) studies reporting on human subjects, and (3) studies using dichotomous clinical outcomes as primary or secondary endpoint. Exclusion criteria were studies reporting on non-pancreatic surgery-related outcomes, using only 
surrogate outcomes as primary and secondary endpoints, describing a study protocol or studies that were published only in abstract form.

\section{Study selection}

One author (M.C.) screened all the retrieved abstracts and crosschecked the reference lists for additional eligible papers. Full texts of potentially eligible RCTs were retrieved via the databases or by contacting the corresponding author. Two authors (M.C., S.C.) performed the full-text examination and decided on inclusion for final analysis. Disagreements on inclusion or exclusion were resolved through discussion with a third author (S.O.D.). The data on study origin, design, sample size, study interval, type of intervention, primary and secondary endpoints, definitions of complications, and trial outcomes were recorded systematically in a database. Extra attention was given to dichotomous endpoints of different RCTs that were published by the same institute using data from overlapping time intervals. These were taken into account only once in order to prevent the use of duplicate data.

Development step 2: reaching consensus on definitions of complications after pancreaticoduodenectomy.

A web survey was used as a tool to reach consensus on which endpoints should be included in the CEP. This web-based survey was designed using free online software (http://www.surveymonkey.com). An example of the survey can be found in Appendix 1 (supporting information). All endpoints used in the included RCTs were systematically registered in a database. The most frequently used endpoints formed the basis of the web survey. The survey consisted of two parts. The aim of Part I was to obtain a first selection of single endpoints to be included in a CEP for PD. Directors of HPB surgery units worldwide were personally contacted by email and invited to participate in the survey. Multiple email reminders were sent in order to acquire a maximum of reactions, comments and opinions. In this part the participants were explicitly asked whether they preferred the use of the Accordion severity grading scale described by Strasberg et al. ${ }^{17,18}$ or the Dindo-Clavien classification as standard complication-grading system. Initial analysis of the survey results revealed a negative opinion toward the adoption of the Accordion grading scale. Thus the Dindo-Clavien severity grading system was used onwards. This system grades complications according to the interventions needed to treat the complication. Second the selected single components were defined (if possible using already existing definitions of known study groups such as the International Study Group of Pancreatic Surgery (ISGPS) ${ }^{19,20}$ and International Study Group of Pancreatic Fistula (ISGPF) ${ }^{21}$ ). The used definitions can be found in Appendix 2 (supporting information). After collection of all replies, the proposed definitions were adjusted according to the suggestions of the responders and selected when a minimum of two thirds of the responders agreed upon the proposed 
definition. Finally, during multiple meetings by the research group a CEP for PD was defined.

Development step 3: selection of components of the pancreaticoduodenectomy-specific endpoint

The second part of the survey covered the components of the PD-specific CEP. These were selected after receiving the responses from part I of the survey. Only complications with a Dindo-Clavien severity grade of 3 or more, and on which more than $75 \%$ of the responders agreed, were included. An agreement percentage of $75 \%$ is commonly used in surveys using the Delphi technique ${ }^{22}$. In part II of the survey this final combination of components was proposed to the respondents as a final endpoint on which agreement should be reached. Again, the final CEP for PD was accepted when more than $75 \%$ of the respondents agreed.

Evaluation of the incidence of the pancreaticoduodenectomy-specific composite endpoint

The incidence of the individual components and the proposed PD-specific CEP was determined using two prospectively collected databases containing a consecutive series of patients having undergone a PD operation in Maastricht University Medical Center between 1 January 2000 and 1 October 2012, and in the Royal Free Hospital London between 1 July 2010 and 30 June 2012. Complications occurring within 90 days of the initial PD operation were recorded and graded according to the Dindo-Clavien classification.

\section{Statistical analysis}

The incidence of the individual components and the proposed PD-specific CEP was determined using two prospectively collected databases containing a consecutive series of patients having undergone a PD operation in Maastricht University Medical Center between 1 January 2000 and 1 October 2012, and in the Royal Free Hospital London between 1 July 2010 and 30 June 2012. Complications occurring within 90 days of the initial PD operation were recorded and graded according to the Dindo-Clavien classification $^{8}$. 


\section{RESULTS}

\section{Identification of single-component clinical endpoints in randomized trials in pancreatic surgery}

A total of 307 abstracts of RCTs that fulfilled the search terms were identified with the electronic search. The main reasons for exclusion are shown in Figure 5.1. Characteristics of the 33 RCTs included in the final analysis are summarized in Table 5.1. The mean sample size of the included RCTs was 123 patients (range 22-450 patients). Table 5.1 shows more detailed information about (1) which single-component clinical endpoints were used most frequently as the primary or secondary endpoint and (2) how many times they were used in the selected RCTs.

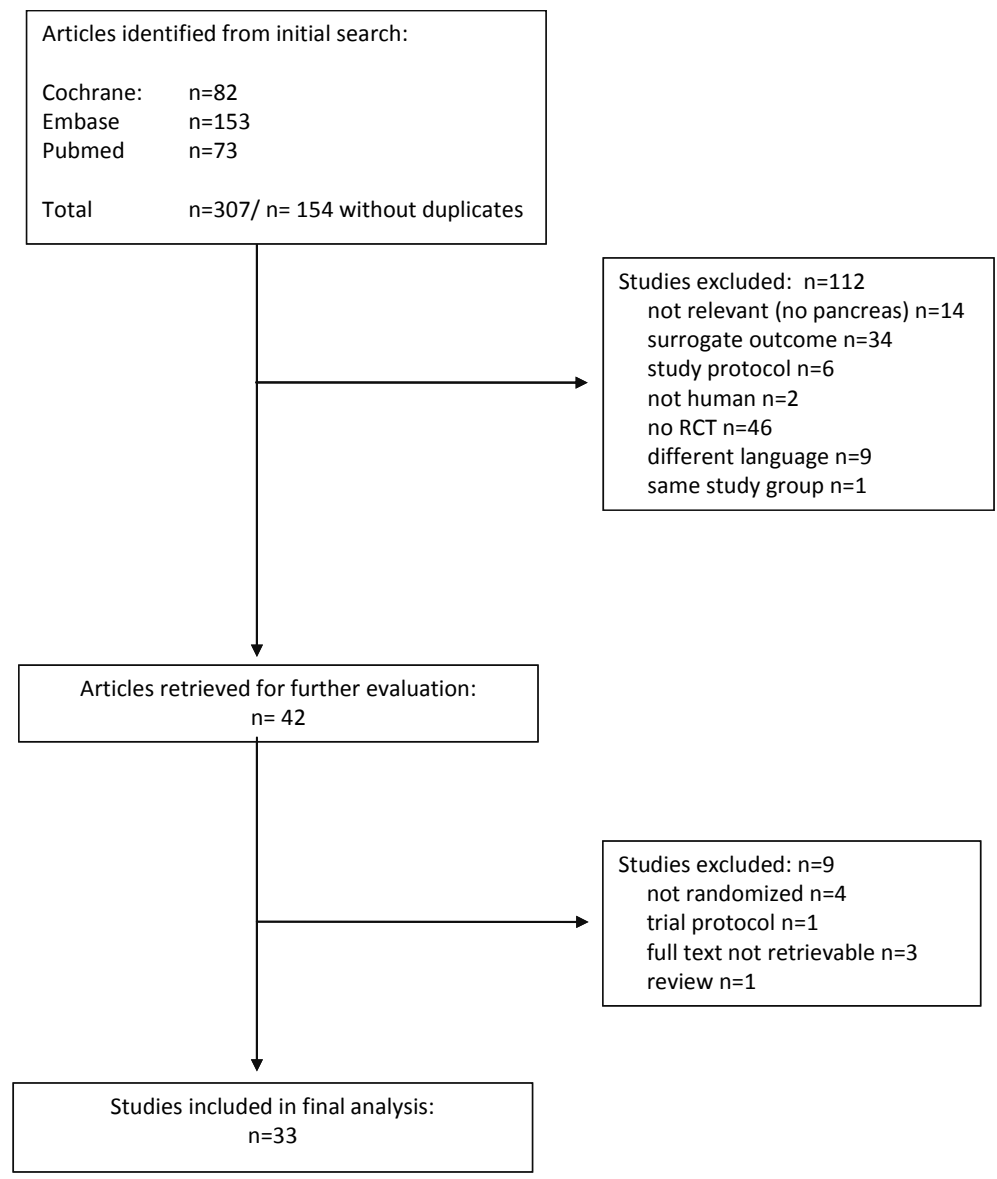

Figure 5.1 Flow chart of study selection 
Table 5.1 Characteristics and single-component clinical endpoints of 33 randomized controlled trials in pancreaticoduodenectomy published between January 2006 and August 2011.

\begin{tabular}{|c|c|}
\hline & No. of trials \\
\hline \multicolumn{2}{|l|}{ Continent of origin } \\
\hline Asia & 15 \\
\hline Europe & 13 \\
\hline North America & 5 \\
\hline \multicolumn{2}{|l|}{ Type of intervention } \\
\hline Drug & 5 \\
\hline Nutrition & 4 \\
\hline Surgical technique & 17 \\
\hline Other & 7 \\
\hline Multicenter trial & 10 \\
\hline Clinical dichotomous primary endpoint & 27 \\
\hline Composite Endpoint & 3 \\
\hline Surrogate continuous primary endpoint & 6 \\
\hline \multicolumn{2}{|c|}{ Single-component clinical outcome used as primary or secondary endpoint } \\
\hline Operative, 30-day, in-hospital mortality & 23 \\
\hline Biliary leakage & 19 \\
\hline Requirement for blood transfusion & 7 \\
\hline Intra-abdominal abscess & 18 \\
\hline Wound infection, wound dehiscence & 20 \\
\hline Intra-abdominal hemorrhage, bleeding, hemoperitoneum & 19 \\
\hline Pneumonia & 12 \\
\hline Ascites & 5 \\
\hline Pleural effusion & 7 \\
\hline Sepsis & 7 \\
\hline Gastrojejunostomy leakage & 11 \\
\hline Pancreaticojejunostomy leakage & 25 \\
\hline Pancreatic fistula & 28 \\
\hline Delayed gastric emptying & 25 \\
\hline Body weight gain within 3 months $(\mathrm{kg})$ & 3 \\
\hline Pancreatic dysfunction & 9 \\
\hline Quality of life & 4 \\
\hline
\end{tabular}

\section{Consensus on definitions of complications after pancreaticoduodenectomy}

Fifty HPB surgeons were invited to participate in the survey. Twenty-five of those surgeons (50\%) completed the survey. Participating surgeons were from Canada (5\%), the United States (5\%), the Netherlands (40\%), and various other European countries (50\%). According to the respondents, the following complications were considered PDspecific complications: intra-abdominal abscess (100\%), bile leakage/hepaticojejunostomy leakage (80\%), sepsis (95\%), post-PD hemorrhage (95\%), gastrojejunostomy leakage (80\%), pancreatic fistula formation (95\%), delayed gastric emptying (90\%), and operative mortality (100\%). Eight complications were considered not to be PD specific (Table 5.2). The participating surgeons agreed with the proposed definitions (Appendix 2). Several respondents recommended changing the term 
"pancreatic fistula" into "leakage of the pancreatic anastomosis". This covers leakage of both the pancreaticojejunostomy and the pancreaticogastrostomy. Following suggestions made by some respondents $(n=3)$, the mortality registration period was changed from 30 to 90 days by consensus.

Table 5.2 Selection of components of the pancreatic surgery-specific composite endpoint

\begin{tabular}{lc}
\hline & $\begin{array}{c}\text { No. of respondents } \\
\text { Indicating that the component should be included in the } \\
\text { pancreatic surgery-specific CEP }(n=25)\end{array}$ \\
\hline Sepsis & $25(100)$ \\
Gastrojejunostomy leakage & $24(96)$ \\
Post pancreatic surgery hemorrhage & $24(96)$ \\
DGE & $24(96)$ \\
Bile leakage/ hepaticojejunostomy leakage & $23(92)$ \\
Pancreatic fistula (pancreaticojejunostomy-, & $21(84)$ \\
pancreaticogastrostomy leakage) & $25(100)$ \\
Mortality & \\
& $25(100)$ \\
Wound infection & $10(40)$ \\
Pleural effusion & $1(4)$ \\
Ascites & $1(4)$ \\
Pneumonia & $8(32)$ \\
Renal failure & $4(16)$ \\
Blood transfusion & $8(32)$ \\
Cost & $3(14)$ \\
Quality of life & $9(36)$ \\
\hline
\end{tabular}

Values in parentheses are percentages; DGE delayed gastric emptying

\section{Selection of components of the pancreaticoduodenectomy-specific composite endpoint}

Twenty-five (100\%) experts who completed part I of the survey also completed part II. Their preferences for components to be included in the PD-specific CEP are summarized in Table 5.2. Final analysis of part II resulted in selection of eight complications with a Dindo-Clavien grade of 3 or higher. All respondents agreed with this proposal, and each respondent indicated that he or she would be interested in using a PD-specific CEP as a primary endpoint of a RCT.

\section{Evaluation of the incidence of the pancreaticoduodenectomy-specific endpoint}

Between 1 January 2000 and 1 October 2012, 387 patients underwent a pancreatic resection at Maastricht University Medical Center, and between 1 July 2010 and 30 June 2012, 178 patients underwent resection at the Royal Free Hospital London. Of these patients, 178 and 120 underwent a PD at the respective institutions. 
The characteristics of the procedures and their postoperative complications are displayed in Table 5.3. At the Maastricht University Medical Center the incidence of individual complications as combined in the CEP and with a Dindo-Clavien grade of 3 or higher within 90 days after PD, varied between $0.5 \%$ for delayed gastric emptying (DGE) and $15.7 \%$ for intra-abdominal abscess. The incidence of the PD-specific CEP was $24.7 \%$ (44 patients). The incidence of the individual components of the CEP at the Royal Free Hospital London ranged from 1.7 to $8.3 \%$ for operative mortality and post-pancreatic surgery intra-abdominal hemorrhage, respectively. The incidence of the PD-specific CEP in this group was $23.3 \%$ (28 patients).

Table 5.3 Patient characteristics and incidence of complications after pancreatic surgery

\begin{tabular}{lcc}
\hline & $\begin{array}{c}\text { Mastricht University } \\
\text { Medical Center } \\
(n=178 \text { resections) }\end{array}$ & $\begin{array}{c}\text { Royal Free Hospital } \\
\text { London } \\
\text { (n=120 resections) }\end{array}$ \\
\hline Sex (F:M) & $29: 111$ & $57: 63$ \\
Age (Years)* & 67 & 62 \\
Pancreaticoduodenectomy (PPPD) & $145(81.5)$ & $20(16.7)$ \\
Pancreaticoduodenectomy (Whipple's procedure) & $33(18.5)$ & $100(83.3)$ \\
Complication grade 1-2 (Dindo-Clavien) & & $6(5.0)$ \\
Intra-abdominal abscess & $4(2.2)$ & $4(3.3)$ \\
Sepsis & $1(0.5)$ & - \\
Gastrojejunostomy leakage & - & $7(5.8)$ \\
Post pancreaticoduodenectomy hemorrhage & - & $2(1.7)$ \\
Bile leakage/hepaticojejunostomy leakage & $2(1.1)$ & $12(10.0)$ \\
Pancreatic anastomosis leakage & $5(2.8)$ & $19(15.8)$ \\
Delayed gastric emptying & $16(8.9)$ & $4(3.3)$ \\
Complication grade 3-5 (Dindo-Clavien) & & $6(5.0)$ \\
Intra-abdominal abscess & $28(15.7)$ & $4(3.3)$ \\
Sepsis & $9(5.0)$ & $10(8.3)$ \\
Gastrojejunostomy leakage & $1(0.5)$ & $4(3.3)$ \\
Post pancreaticoduodenectomy hemorrhage & $7(3.9)$ & $7(5.8)$ \\
Bile leakage/hepaticojejunostomy leakage & $7(3.9)$ & $5(4.1)$ \\
Pancreatic anastomosis leakage & $24(13.4)$ & $2(1.7)$ \\
Delayed gastric emptying & $1(0.5)$ & $28(23.3)$ \\
Operative mortality 90 days & $10(5.6)$ & $44(24.7)$ \\
Pancreaticoduodenectomy specific CEP & & \\
\hline
\end{tabular}

Values in parentheses are in percentages unless indicated otherwise; * values are in mean

Subsequently, taking only complications with Dindo-Clavien grade 3 or higher in the present data set into account, the theoretical sample size for a RCT was calculated with the goal of showing a relative reduction in the individual complication or the PD-specific CEP by one third, one half, or two thirds (Table 5.4). To show a risk reduction of 33, 50, and $66 \%$, the sample size of an RCT powered on the incidence of the PD-specific CEP reached a number of 386,154 , and 80 , respectively, based on the Maastricht University Medical Center patient cohort. Calculation of the relative risk reduction based on the Royal Free Hospital London data set showed PD-specific CEP sample sizes of 414, 165, 86 , respectively. 
Table 5.4 Sample size of each arm of an adequately powered randomized controlled trial aiming to show a one-third, one-half or two-thirds relative reduction in the primary endpoint

\begin{tabular}{|c|c|c|c|c|c|c|}
\hline & \multicolumn{6}{|c|}{ Relative reduction in primary endpoint } \\
\hline & \multicolumn{3}{|c|}{ Maastricht University Medical Center } & \multicolumn{3}{|c|}{ Royal Free Hospital London } \\
\hline & $33 \%$ & $50 \%$ & $66 \%$ & $33 \%$ & $50 \%$ & $66 \%$ \\
\hline Intra-abdominal abscess & 665 & 264 & 136 & 3546 & 1391 & 714 \\
\hline Sepsis & 2306 & 906 & 466 & 2306 & 906 & 466 \\
\hline Gastrojejunostomy leakage & 23976 & 9384 & 4813 & 3546 & 1391 & 714 \\
\hline $\begin{array}{l}\text { Post-pancreaticoduo- } \\
\text { denectomy hemorrhage }\end{array}$ & 2985 & 1171 & 602 & 1344 & 529 & 273 \\
\hline $\begin{array}{l}\text { Bile leakage/ hepatico- } \\
\text { jejunostomy leakage }\end{array}$ & 2985 & 1171 & 602 & 3546 & 1391 & 714 \\
\hline $\begin{array}{l}\text { Pancreatic anastomosis } \\
\text { leakage }\end{array}$ & 2985 & 315 & 163 & 1974 & 776 & 399 \\
\hline Delayed gastric emptying & 23976 & 9384 & 4813 & 2785 & 1093 & 562 \\
\hline Operative mortality & 2048 & 805 & 414 & 7107 & 2784 & 1429 \\
\hline $\begin{array}{l}\text { Pancreaticoduodenectomy } \\
\text { specific CEP }\end{array}$ & 386 & 154 & 80 & 414 & 165 & 86 \\
\hline
\end{tabular}

Assumptions: $\alpha=0.05, \beta=0.20$ and two-sided testing. CEP, composite endpoint

\section{DISCUSSION}

The aim of the present study was to design a consensus-based CEP for PD that can be used as a primary endpoint in RCTs on PD in order to reach adequate power and to improve comparability between studies. The PD-specific CEP developed in this study consists of 8 components: intra-abdominal abscess, sepsis, gastrojejunostomy leakage, bile leakage, leakage of the pancreatic anastomosis, delayed gastric emptying, post-PD hemorrhage, and mortality occurring within 90 days postoperatively. Additionally, the aforementioned complications need to have a Dindo-Clavien grade of 3 or more. The PD-specific CEP was evaluated in two cohorts of patients undergoing PD in two tertiary referral centers in Europe, Maastricht and London. The incidence of the CEP in both cohorts was considerably higher than the incidence of the individual components. As a result, the theoretical sample size of a RCT in PD decreased substantially and will result in a higher statistical precision and efficiency.

CEPs have been used commonly in cardiovascular trials and pharmacological trials ${ }^{12,23}$. Publication of these trials has resulted in criticism of the drawbacks of CEPs. For example, individual components of a CEP may be ill defined, may differ in severity or incidence, and might not be affected in the same manner by the intervention. For these reasons, study groups have set up recommendations for the use of composite outcomes $^{7,24-26}$.

In the present study an attempt was made to address these issues in a systematic way. To gain international consensus regarding definitions of most of the individual components, the use of definitions already developed by international study groups 
such as the ISGPS/ISGPF was suggested ${ }^{19-21}$. After evaluation and adjustments, international consensus was reached regarding individual components that had not been defined previously (Appendix 2). Furthermore, in order to include components of similar severity and clinical relevance, only components with a Dindo-Clavien score of grade 3 or more were used in the PD-specific CEP. The Dindo-Clavien classification aims to objectively group different complications with respect to the severity of therapeutic interventions. Importantly, the use of a standardized complication classification system like the Dindo-Clavien system increases comparability between studies.

In the present study, experts were asked whether quality of life (QoL) should be included in the CEP, but QoL was not considered to be sufficiently PD-specific. Hence, patient perceptions were not included in this CEP. Nevertheless, they may be of importance, as certain complications can have serious implications for patients, and patients may view the consequences of these complications (e.g. pain, fatigue, confusion) as more critical. The OMERACT initiative (Outcome Measures in Rheumatoid Arthritis Clinical Trial) introduced the development of a COS, which also includes patient perception ${ }^{27}$. More recently, the COMET initiative (Core Outcome Measures in Effectiveness Trials) extended this approach in order to develop COSs that are applicable to other disciplines as well, such as pediatrics and bariatric surgery ${ }^{11,28}$. At present, the senior author's group is developing a COS for pancreatic surgery in collaboration with the COMET initiative.

The mean sample size of RCTs involving pancreatic surgery was found to be 123 after a systematic literature search. However, sample sizes calculated for single-component primary outcomes, as used in the included studies, should be much higher. This was shown in a previous publication where sample sizes were calculated for a singlecomponent primary endpoint for liver surgery (range 826-6250 patients) having complication incidences comparable to those associated with pancreatic surgery ${ }^{29}$. As a consequence, the studies included in this systematic review were most likely underpowered. Also, only a few of the included studies, those that were large and multicenter, using a dichotomous primary endpoint showed a statistical significant difference in outcome. This present study also shows that it is almost impossible to perform future studies on PD that are adequately powered on a single primary outcome as it would need sample sizes that are too large to be reached for a single institution.

Only three of the included studies used a CEP as primary endpoint, and two of them defined the CEP as "overall and pancreas-specific postoperative complications" or "serious complications"15,16. However "overall complications" as a CEP should be avoided, because complications with less clinical relevance may contribute strongly to the incidence of the CEP ${ }^{25}$. For this reason, it is important to explicitly define each of the CEP components and assess their severity. 
A limitation of our study is the selection bias introduced by inviting specific surgeons to the web survey. The majority of respondents came from Europe. This means that the CEP to be used in RCTs is mainly based on the view of European pancreatic surgeons. In this study a CEP is developed specifically for PD instead of a CEP for pancreatic surgery in general. PD is a procedure that differs considerably from other types of pancreatic surgery because it involves the construction of multiple anastomoses. This is not necessary, for example, for a distal pancreatectomy. The anastomoses are the primary cause of specific PD complications, such as anastomotic leakage and post-PD hemorrhage, and PD therefore should not be lumped together with other surgical procedures of the pancreas.

It may be necessary to develop CEPs for other forms of pancreatic surgery, as well as for drug-related pancreatic research. Furthermore, weighing of specific components of a CEP, not only by clinicians but also by patients, might be considered in future work. This issue will also be addressed in the development of the COS for pancreatic surgery by the present authors' group.

In this study a CEP for PD was developed in a systematic fashion, taking into account the definition of individual components as well as the clinical relevance of included components. Given the positive results of this study, a prospective validation of the PDspecific CEP for RCTs worldwide may be the appropriate next step. 


\section{REFERENCES}

1. Kaido T. Analysis of randomized controlled trials on hepatopancreatic surgery. Dig Dis Sci 2006;51: 1761-1766.

2. Kaido T. Recent randomized controlled trials in pancreaticoduodenectomy. Pancreas 2006;33:228-232.

3. Winter JM, Cameron JL, Campbell KA, Arnold MA, Chang DC, Coleman J, Hodgin MB, Sauter PK, Hruban RH, Riall TS, Schulick RD, Choti MA, Lillemoe KD, Yeo CJ. 1423 pancreaticoduodenectomies for pancreatic cancer: a single-institution experience. J Gastrointest Surg 2006;10:1199-1210.

4. DeOliveira ML, Winter JM, Schafer M, Cunningham SC, Cameron JL, Yeo CJ, Clavien PA. Assessment of complications after pancreatic surgery: a novel grading system applied to 633 patients undergoing pancreaticoduodenectomy. Ann Surg 2006; 244:931-937.

5. de Wilde RF, Besselink MG, van der Tweel I, de Hingh IH, van Eijck CH, Dejong CH, Porte RJ, Gouma DJ, Busch OR, Molenaar IQ; Dutch Pancreatic Cancer Group. Impact of nationwide centralization of pancreaticoduodenectomy on hospital mortality. Br J Surg 2012;99:404-410.

6. Washburn K. Endpoints in trials for clinical liver trans- plantation. Curr Opin Organ Transplant 2008; 13:252-256.

7. Freemantle N, Calvert M, Wood J, Eastaugh J, Griffin C. Composite out- comes in randomized trials: greater precision but with greater uncertainty? JAMA 2003;289:2554-2559.

8. van den Broek MA, van Dam RM, van Breukelen GJ, Bemelmans MH, Oussoultzoglou E, Pessaux P, Dejong $\mathrm{CH}$, Freemantle N, Olde Damink SW. Development of a composite endpoint for randomized controlled trials in liver surgery. Br J Surg 2011;98:1138-1145.

9. TIME Investigators. Trial of invasive versus medical therapy in elderly patients with chronic symptomatic coronary-artery disease (TIME): a randomised trial. Lancet 2001;358:951-957.

10. Niewoehner DE, Erbland ML, Deupree RH, Collins D, Gross NJ, Light RW, Anderson P, Morgan NA. Effect of systemic glucocorticoids on exacerbations of chronic obstructive pulmonary disease. Department of Veterans Affairs Cooperative Study Group. N Engl J Med 1999;340:1941-1947.

11. Sinha IP, Gallagher R, Williamson PR, Smyth RL. Development of a core outcome set for clinical trials in childhood asthma: a survey of clinicians, parents, and young people. Trials 2012;13:103.

12. Taylor DW, Barnett HJ, Haynes RB, Ferguson GG, Sackett DL, Thorpe KE, Simard D, Silver FL, Hachinski V, Clagett GP, Barnes R, Spence JD. Low-dose and high-dose acetylsalicylic acid for patients undergoing carotid endarterectomy: a randomised controlled trial. ASA and Carotid Endarterectomy (ACE) Trial Collaborators. Lancet 1999;353: 2179-2184.

13. Yusuf S, Sleight $P$, Pogue J, Bosch J, Davies R, Dagenais G. Effects of an angiotensin- converting-enzyme inhibitor, ramipril, on cardiovascular events in high-risk patients. The Heart Outcomes Prevention Evaluation Study Investigators. N Engl J Med 2000;342:145-153.

14. Diener MK, Seiler CM, Rossion I, Kleeff J, Glanemann M, Butturini G, Tomazic A, Bruns CJ, Busch OR, Farkas S, Belyaev O, Neoptolemos JP, Halloran C, Keck T, Niedergethmann M, Gellert K, Witzigmann H, Kollmar O, Langer P, Steger U, Neudecker J, Berrevoet F, Ganzera S, Heiss MM, Luntz SP, Bruckner T, Kieser M, Büchler MW. Efficacy of stapler versus hand-sewn closure after distal pancreatectomy (DISPACT): a randomised, controlled multicentre trial. Lancet 2011;377: 1514-1522.

15. Ramos-De la Medina A, Sarr MG. Somatostatin analogues in the prevention of pancreas-related complications after pancreatic resection. J Hepatobiliary Pancreat Surg 2006;13:190-193.

16. van der Gaag NA, Rauws EA, van Eijck CH, Bruno MJ, van der Harst E, Kubben FJ, Gerritsen JJ, Greve JW, Gerhards MF, de Hingh IH, Klinkenbijl JH, Nio CY, de Castro SM, Busch OR, van Gulik TM, Bossuyt PM, Gouma DJ. Pre-operative biliary drainage for cancer of the head of the pancreas. N Engl J Med 2010;362:129-137.

17. Dindo D, Demartines N, Clavien PA. Classification of surgical complications: a new proposal with evaluation in a cohort of 6336 patients and results of a survey. Ann Surg 2004;240:205-213.

18. Strasberg SM, Linehan DC, Hawkins WG. The accordion severity grading system of surgical complications. Ann Surg 2009;250:177-186. 
19. Wente MN, Bassi C, Dervenis C, Fingerhut A, Gouma DJ, Izbicki JR, Neoptolemos JP, Padbury RT, Sarr MG, Traverso LW, Yeo CJ, Büchler MW. Delayed gastric emptying (DGE) after pancreatic surgery: a suggested definition by the International Study Group of Pancreatic Surgery (ISGPS). Surgery 2007;142: 761-768.

20. Welsch T, Eisele H, Zschäbitz S, Hinz U, Büchler MW, Wente MN. Critical appraisal of the International Study Group of Pancreatic Surgery (ISGPS) consensus definition of postoperative hemorrhage after pancre- atoduodenectomy. Langenbecks Arch Surg 2011;396:783-791.

21. Bassi C, Dervenis C, Butturini G, Fingerhut A, Yeo C, Izbicki J, Neoptolemos J, Sarr M, Traverso W, Buchler M; International Study Group on Pancreatic Fistula Definition. Postoperative pancreatic fistula: an international study group (ISGPF) definition. Surgery 2005;138:8-13.

22. Houwink EJ, Henneman L, Westerneng M, van Luijk SJ, Cornel MC, Dinant JG, Vleuten Cv. Prioritization of future genetics education for general practitioners: a Delphi study. Genet Med 2012;14:323-329.

23. Gerstein HC, Yusuf S, Bosch J, Pogue J, Sheridan P, Dinccag N, Hanefeld M, Hoogwerf B, Laakso M, Mohan V, Shaw J, Zinman B, Holman RR. Effect of rosiglitazone on the frequency of diabetes in patients with impaired glucose tolerance or impaired fasting glucose: a randomised controlled trial. Lancet 2006;368:1096-1105.

24. Ferreira-González I, Busse JW, Heels-Ansdell D, Montori VM, AkI EA, Bryant DM, Alonso-Coello P, Alonso J, Worster A, Upadhye S, Jaeschke R, Schünemann HJ, Permanyer-Miralda G, Pacheco-Huergo V, Domingo-Salvany $\mathrm{A}, \mathrm{Wu} \mathrm{P}$, Mills EJ, Guyatt GH. Problems with use of composite end points in cardiovascular trials: systematic review of randomised controlled trials. BMJ 2007;334:786.

25. Ferreira-González I, Permanyer-Miralda G, Busse JW, Bryant DM, Montori VM, Alonso-Coello P, Walter $\mathrm{SD}$, Guyatt GH. Methodologic discussions for using and interpreting composite endpoints are limited, but still identify major concerns. J Clin Epidemiol 2007;60: 651-657.

26. Tomlinson G, Detsky AS. Composite end points in randomized trials: there is no free lunch. JAMA 2010; 303:267-268.

27. Tugwell $P$, Boers $M$, Brooks $P$, Simon L, Strand V, Idzerda L. OMERACT: an international initiative to improve outcome measurement in rheumatology. Trials 2007;8:38.

28. http://blogs.bmj.com/bmj/2012/08/01/james-hopkins-and-jane-blazeby-development-of-a-coreoutcome- set-for-bariatric-surgery/. Accessed 21 Dec 2013

29. van den Broek MA, van Dam RM, Malagó $\mathrm{M}$, Dejong $\mathrm{CH}$, van Breukelen GJ, Olde Damink SW. Feasibility of randomized controlled trials in liver surgery using surgery-related mortality or morbidity as endpoint. Br J Surg 2009;96:1005-1014. 


\section{PART $\mid$}

Development of ERAS guidelines and a multicenter trial for pancreaticoduodenectomy 


\section{CHAPTER}

Guidelines for perioperative care for

pancreaticoduodenectomy: Enhanced Recovery After

Surgery (ERAS ${ }^{\oplus}$ ) Society recommendations

K Lassen, MME Coolsen, K Slim, F Carli, JE de Aguilar-Nascimento, M Schäfer, RW Parks, $\mathrm{KCH}$ Fearon, DN Lobo, N Demartines, M Braga, O Ljungqvist and CHC Dejong, on behalf of the ERAS Society, the European Society for Clinical Nutrition and Metabolism and the International Association for Surgical Nutrition and Metabolism

Clin Nutr. 2012;31:817-830 \& World J Surg. 2013;37:240-258 


\section{ABSTRACT}

Background \& aims

Protocols for enhanced recovery provide comprehensive and evidence-based guidelines for best perioperative care. Protocol implementation may reduce complication rates and enhance functional recovery and, as a result of this, also reduce length-of-stay in hospital. There is no comprehensive framework available for pancreaticoduodenectomy.

\section{Methods}

An international working group constructed within the Enhanced Recovery After Surgery (ERAS ${ }^{\circledR}$ ) Society constructed a comprehensive and evidence-based framework for best perioperative care for pancreaticoduodenectomy patients. Data were retrieved from standard databases and personal archives. Evidence and recommendations were classified according to the GRADE system and reached through consensus in the group. The quality of evidence was rated "high", "moderate", "low" or "very low". Recommendations were graded as "strong" or "weak".

Results

Comprehensive guidelines are presented. Available evidence is summarized and recommendations given for 27 care items. The quality of evidence varies substantially and further research is needed for many issues to improve the strength of evidence and grade of recommendations.

\section{Conclusions}

The present evidence-based guidelines provide the necessary platform upon which to base a unified protocol for perioperative care for pancreaticoduodenectomy. A unified protocol allows for comparison between centers and across national borders. It facilitates multi-institutional prospective cohort registries and adequately powered randomized trials. 


\section{INTRODUCTION}

Enhanced Recovery After Surgery (ERAS), Fast-Track or Clinical Pathway programs are multimodal strategies that aim to attenuate the loss of, and improve the restoration of, functional capacity after surgery. Morbidity is reduced ${ }^{1}$ and recovery enhanced by reducing surgical stress, by optimal control of pain, early oral diet and early mobilization. As a consequence, length-of-stay in hospital (LOSH) and costs are also reduced. The ERAS group has published evidence-based consensus recommendations for colorectal surgery ${ }^{2,3}$. Beneficial experiences with clinical pathway programs after pancreaticoduodenectomy (PD, Whipple's procedure) have been published ${ }^{4-9}$, but the reported series employed different protocols, or no prospective protocol at all ${ }^{6}$. A comprehensive consensus framework is presented on which to base a future protocol for optimal perioperative care after PD. Such a recommendation will allow for a unified protocol to be developed and validated prospectively across different institutions and healthcare systems. This guideline framework has been formulated and endorsed by the ERAS Society, European Society for Clinical Nutrition and Metabolism (ESPEN) and the International Association for Surgical Metabolism and Nutrition (IASMEN).

\section{METHODS}

\section{Literature search}

The authors met in April 2011 and the topics to be included were agreed and allocated. A principal literature search up to June 2011 was undertaken. Comprehensive drafts were circulated for discussion and reviewed in a group conference in November 2011. Additional relevant literature published after June 2011 was considered by members of the group at meetings in November 2011 and May 2012.

\section{Study selection}

All co-authors screened web-based databases and personal archives for relevant articles. Non-systematic emphasis was given to more recent publications and publications of better quality (moderate- and high-quality randomized controlled trials and high-quality, large cohort studies; and systematic reviews and meta-analyses of these). Retrospective series were considered only if data of better quality could not be identified.

\section{Quality assessment and grading}

The strength of evidence and conclusive recommendations were assessed and agreed by all authors in May 2012. Quality of evidence and recommendations were evaluated 
according to the Grading of Recommendations, Assessment, Development and Evaluation (GRADE) system ${ }^{10-12}$. Quoting from the GRADE guidelines, $^{12}$ the recommendations are: "Strong recommendations indicate that the panel is confident that the desirable effects of adherence to a recommendation outweigh the undesirable effects". "Weak recommendations indicate that the desirable effects of adherence to a recommendation probably outweigh the undesirable effects, but the panel is less confident". Recommendations are based on quality of evidence (high, moderate, low, very low) but also on the balance between desirable and undesirable effects; and on values and preferences ${ }^{12}$. The latter implies that, in some cases, strong recommendations may be reached from low-quality data and vice versa. A summary of the guidelines is shown in Table 6.1.

\section{EVIDENCE AND RECOMMENDATIONS}

\section{Preoperative counseling}

Preoperative counseling targeting expectations about surgical and anesthetic procedures may diminish fear and anxiety and enhance postoperative recovery and discharge. ${ }^{13-15}$ Personal counseling, leaflets or multimedia information containing explanations of the procedure along with tasks that the patient should be encouraged to fulfill may improve perioperative feeding, early postoperative mobilization, pain control, and respiratory physiotherapy; and hence reduce the risk of complications ${ }^{16-18}$. Ideally, the patient should meet with the surgeon, anesthetist and nurse.

Summary and recommendation: Patients should receive dedicated preoperative counseling routinely.

Evidence level: Low.

Recommendation grade: Strong.

\section{Preoperative biliary drainage}

Five meta-analyses ${ }^{19-23}$, and two articles from a randomized controlled trial (RCT) not included in the meta-analyses ${ }^{24,25}$, assessed the role of biliary drainage before PD. The first meta-analysis from $2002^{19}$, included randomized $(n=5)$ and non- randomized trials $(n=18)$. A Cochrane review ${ }^{21}$ included 5 randomized trials, but considered all 5 trials to have a risk of bias, thereby weakening the conclusions reached. Of the trials included, 4 evaluated percutaneous drainage and 1 evaluated endoscopic drainage. The Cochrane review concluded that preoperative biliary drainage did not decrease mortality in patients with obstructive jaundice. 


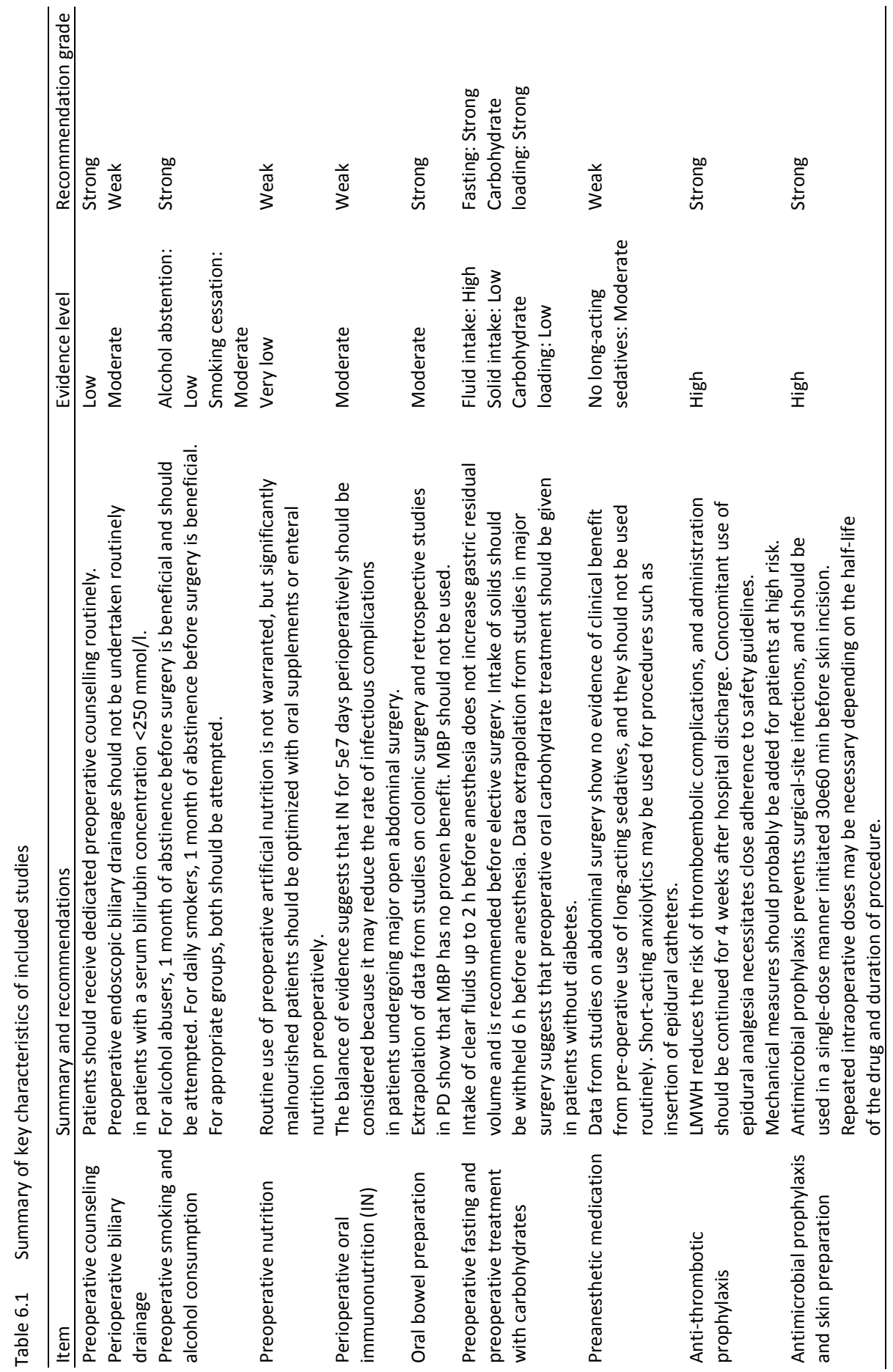




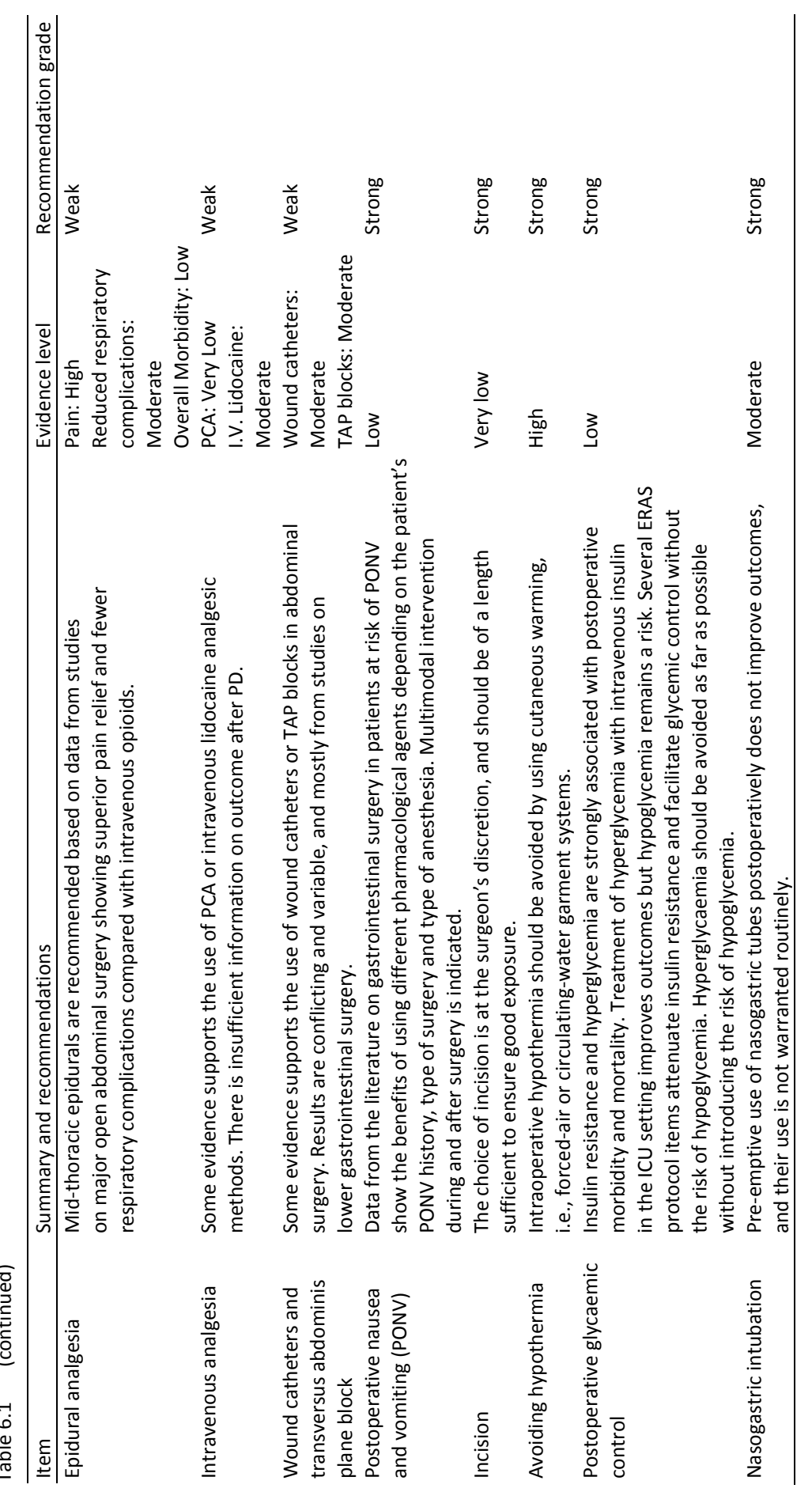




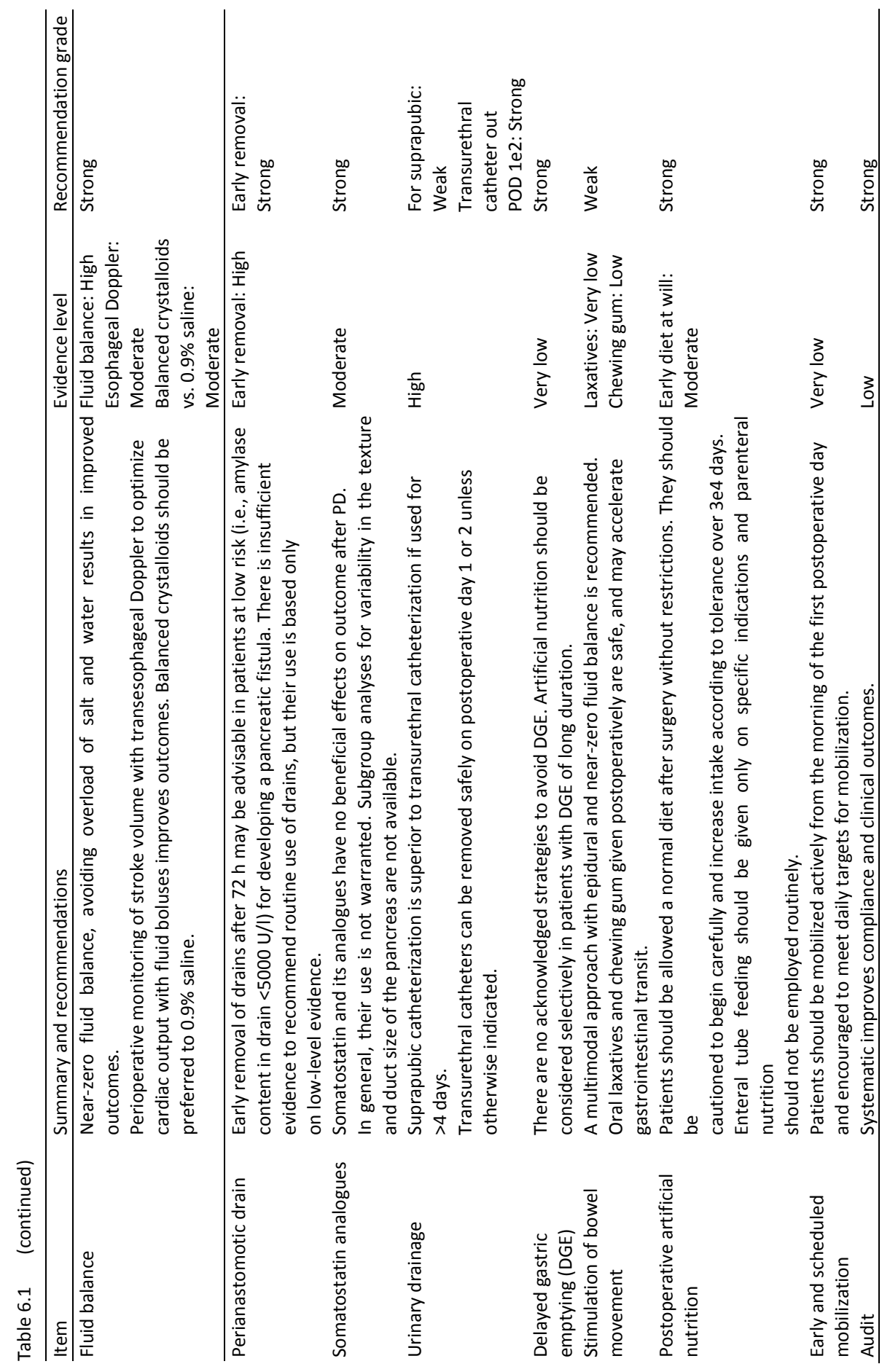


Although there was a trend towards decreased postoperative morbidity, the increased risk of procedure-related complications counterbalanced this possible benefit (especially for percutaneous drainage). The findings of the Cochrane review were in accordance with those of the other meta-analyses, suggesting that preoperative drainage confers neither benefit nor harm. One recent RCT not included in the metaanalyses $^{24}$ (and which included patients with serum bilirubin concentrations $<250 \mathrm{mmol} / \mathrm{l}$ ) showed increased morbidity in patients undergoing preoperative biliary drainage (endoscopic primarily; percutaneous as rescue option), but the delay in surgery did not affect overall survival ${ }^{25}$.

Summary and recommendation: Preoperative endoscopic biliary drainage should not be carried out routinely in patients with a serum bilirubin concentration $<250 \mathrm{mmol} / \mathrm{l}$.

Evidence level: Moderate.

Recommendation grade: Weak.

\section{Preoperative smoking and alcohol consumption}

Overall postoperative morbidity is increased by two- to three-fold in alcohol abusers ${ }^{26}$. Also, 1 month of preoperative abstinence has been shown to significantly improve outcome in a group who took "five or more drinks (60 g of ethanol) a day without clinical or historical evidence of alcohol related illness" ${ }^{27}$.

Daily smokers ( $>2$ cigarettes daily for $\geq 1$ year) have an increased risk of pulmonary and wound complications ${ }^{28,29}$. RCTs have demonstrated reductions in the rates of both types of complications 1 month after cessation of smoking ${ }^{29,30}$.

Summary and recommendation: For alcohol abusers, 1 month of abstinence before surgery is beneficial and should be attempted.

For daily smokers, 1 month of abstinence before surgery is beneficial. For appropriate groups, both should be attempted.

Evidence level: Alcohol abstention: Low.

Smoking cessation: Moderate.

Recommendation grade: Strong.

\section{Preoperative nutrition}

In western countries, patients scheduled for PD are, in general, not malnourished, and usually present with $<7 \%$ weight loss ${ }^{31}$. In such cases, preoperative artificial nutrition is not warranted. The situation may be different in other regions. It is widely accepted that significantly malnourished patients suffer increased postoperative morbidity after major surgery ${ }^{32-34}$. Preoperative supplements with oral sip feeds or enteral tube feeds are usually administered in these cases, but scientific evidence to support this routine 
(as opposed to no nutritional support) is lacking. Extrapolating data from studies in the postoperative setting suggests that parenteral nutrition should be used only if the enteral route is inaccessible.

Summary and recommendation: Routine use of preoperative artificial nutrition is not warranted, but significantly malnourished patients should be optimized with oral supplements or enteral nutrition preoperatively.

Evidence level: Very low.

Recommendation grade: Weak.

\section{Perioperative oral immunonutrition (IN)}

The role of IN has been investigated thoroughly over many years. Few studies specifically address IN for PD patients, and the variation in active immune-modulating nutrients administered makes interpretation difficult. A reduction in the prevalence of infectious complications is a consistent finding in patients with gastrointestinal cancer, as are beneficial effects on surrogate endpoints (levels of interleukins and C-reactive protein (CRP)) or LOSH. A reduction in mortality has not been shown. Several recently published reviews and meta-analyses ${ }^{35-41}$ conclude that there is a benefit from perioperative and postoperative IN in patients undergoing major gastrointestinal surgery, but results remain inconsistent ${ }^{42-44}$. Beneficial outcomes have been shown in a systematic review of 35 trials in patients undergoing elective surgery, in which argininesupplemented diets were associated with a significantly reduced prevalence of infectious complications and $\mathrm{LOSH}^{45}$. There is also evidence to suggest that immunemodulating nutrition may be more beneficial in undernourished rather than in normally nourished patients. However, IN could be detrimental in patients with sepsis ${ }^{46}$. There are no trials investigating IN within ERAS care pathways.

Summary and recommendation: The balance of evidence suggests that IN for 5-7 days perioperatively should be considered because it may reduce the prevalence of infectious complications in patients undergoing major open abdominal surgery.

Evidence level: Moderate.

Recommendation grade: Weak.

\section{Oral bowel preparation}

Mechanical bowel preparation (MBP) may lead to dehydration and offset fluid and electrolyte balance, particularly in the elderly ${ }^{47}$. Meta-analyses from colonic surgery have not shown clinical benefit from $\mathrm{MBP}^{48,49}$. A large and recent retrospective analysis of 200 consecutive patients undergoing PD did not find any benefit of MBP to a clear 
liquid diet the day before surgery ${ }^{50}$. No trial has compared MBP to a regimen without MBP and an unrestricted diet until midnight before surgery.

Summary and recommendation: Extrapolation of data from colonic surgery and retrospective studies in PD show that MBP has no proven benefit. MBP should not be used.

Evidence level: Moderate.

Recommendation grade: Strong.

\section{Preoperative fasting and preoperative treatment with carbohydrates}

Fasting from midnight has been standard practice in elective surgery, but is not supported by evidence ${ }^{51}$. Overnight fasting increases insulin resistance and discomfort after abdominal surgery ${ }^{52,53}$. Guidelines recommend the intake of clear fluids $\leq 2 \mathrm{~h}$ before the induction of anesthesia as well as a fasting period of $6 \mathrm{~h}$ for solids ${ }^{54}$. The latter recommendation has a weak scientific basis ${ }^{55}$. Intake of a complex clear carbohydrate-rich drink designed for preoperative use $\leq 2 \mathrm{~h}$ before the induction of anesthesia has been shown to reduce hunger, thirst and anxiety, and to decrease postoperative insulin resistance ${ }^{56-58}$. Earlier resumption of gut function after colorectal surgery has also been suggested ${ }^{59}$, and an RCT including some PD patients concluded that oral carbohydrate treatment may preserve skeletal muscle mass ${ }^{60}$. An RCT conducted in patients undergoing cholecystectomy did not show any benefit ${ }^{61}$. Data on the safety and clinical benefit of preoperative carbohydrate in patients with diabetes are sparse ${ }^{62,63}$, and further research is warranted in this group.

Summary and recommendation: Intake of clear fluids up to $2 \mathrm{~h}$ before anesthesia does not increase gastric residual volume and is recommended before elective surgery. Intake of solids should be withheld $6 \mathrm{~h}$ before anesthesia. Data extrapolation from studies in major surgery suggests that preoperative oral carbohydrate treatment should be given in patients without diabetes.

Evidence level: Fluid intake: High.

Solid intake: Low.

Carbohydrate loading: Low.

Recommendation grade: Fasting: Strong.

Carbohydrate loading: Strong.

\section{Pre-anesthetic medication}

Anxiety makes postoperative pain more difficult to control. Pre-emptive treatment of anxiety could lower pain scores and reduce the demand for opiates ${ }^{64}$. However, preinduction anxiolytic medication increases postoperative sedation ${ }^{65}$, and a meta-analysis did not demonstrate reduced postoperative pain with pre-emptive use of analgesics ${ }^{66}$. 
Short-acting anxiolytics may be helpful in some patients during placement of an epidural catheter, and experiences from day surgery suggest that cognitive function is not significantly impaired ${ }^{67}$. Additionally, oral fluids and a carbohydrate-rich beverage have been shown to reduce preoperative anxiety ${ }^{57}$. Medications for chronic pain need to be continued on the morning of surgery, and should be prescribed in the postoperative period.

Summary and recommendation: Data from studies on abdominal surgery show no evidence of clinical benefit from preoperative use of long-acting sedatives, and they should not be used routinely. Short-acting anxiolytics may be used for procedures such as insertion of epidural catheters.

Evidence level: No long-acting sedatives: Moderate.

Recommendation grade: Weak.

\section{Anti-thrombotic prophylaxis}

Malignant disease and major surgery increase the risk of venous thromboembolism $(\mathrm{VTE})^{68}$. Unfractionated and fractionated low- dose heparins are effective at preventing $\mathrm{VTE}^{69}$. Fractionated low- molecular-weight heparin (LMWH) is preferable in view of compliance (once-daily administration) ${ }^{70}$. Treatment is usually initiated 2-12 $\mathrm{h}$ before surgery and continued until patients are fully mobile. A meta-analysis supports continued treatment for 4 weeks after hospital discharge ${ }^{72}$. Concomitant use of LMWH and epidural catheters is controversial ${ }^{72-75}$. It has, therefore, been recommended that the catheter be inserted $\geq 12 \mathrm{~h}$ after a dose of $\mathrm{LMWH}$, and removed $\geq 12 \mathrm{~h}$ after administration of $\mathrm{LMWH}^{76}$. The risk of an epidural or spinal hematoma is increased in patients who are also on anti-platelet drugs or oral anticoagulants ${ }^{73}$. Combined prophylactic modalities have been shown to be superior to pharmacological measures only in preventing VTE ${ }^{77}$. Mechanical intermittent pneumatic leg compression ${ }^{77}$, and elastic stockings may be used as adjuncts in patients who are at moderate or high risk for $\mathrm{VTE}^{78}$.

Summary and recommendation: LMWH reduces the risk of thromboembolic complications. Administration should be continued for 4 weeks after hospital discharge. Concomitant use of epidural analgesia necessitates close adherence to safety guidelines. Mechanical measures should probably be added for patients at high risk.

Evidence level: High.

Recommendation grade: Strong. 


\section{Antimicrobial prophylaxis and skin preparation}

There is ample evidence favoring the prescription of antimicrobial prophylaxis for major abdominal procedures ${ }^{79,80}$. Trials specifically targeting patients undergoing PD were not identified. Recently published studies reported or recommended prescription in a single-dose manner ${ }^{80}$. However, an extra dose should be provided every 3-4 $\mathrm{h}$ during the procedure if drugs with a short half-life are chosen ${ }^{81}$. Initial administration should be as near as possible to the skin incision and $\leq 1 \mathrm{~h}$ before the incision ${ }^{79,82}$. The choice of antibiotic is dependent upon local guidelines, and should be different from the drug of choice for treatment of established infections. Skin preparation with a scrub of chlorhexidine-alcohol has recently been claimed to be superior to povidone-iodine in preventing surgical-site infections ${ }^{83}$. However, the difference is likely to be very small because excellent results are obtained with povidone-iodine ${ }^{84}$. Alcohol-based scrubs have been reported to be used in fire-based and burn injuries ${ }^{85}$.

Summary and recommendation: Antimicrobial prophylaxis prevents surgical-site infections and should be used in a single- dose manner initiated 30-60 min before skin incision. Repeated intraoperative doses may be necessary depending on the half- life of the drug and duration of the procedure.

Evidence level: High.

Recommendation grade: Strong.

\section{Epidural analgesia}

A meta-analysis showed that continuous epidural analgesia with or without opioids provided significant improvement in postoperative pain control compared with parenteral opioids in open abdominal surgery ${ }^{86}$. Moreover, a Cochrane review demonstrated that continuous epidural analgesia is superior to patient-controlled intravenous opioid analgesia in relieving pain $\leq 72 \mathrm{~h}$ after open abdominal surgery ${ }^{87}$. A decreased prevalence of ileus was found for epidural administration of local anesthetic after laparotomy compared with systemic or epidural opioids in one Cochrane review ${ }^{88}$. With respect to complications after abdominal or thoracic surgery, a meta-analysis ${ }^{89}$ concluded that epidural analgesia was associated with a significantly decreased risk of postoperative pneumonia, as well as an improvement in pulmonary function and arterial oxygenation. Also, the use of epidurals has been shown to reduce insulin resistance $^{90}$. Despite the widespread use of epidural analgesia after pancreatic surgery $^{91}$, RCTs that specifically examine the outcomes of epidural analgesia after pancreatic surgery are lacking. A retrospective study comparing epidural analgesia with intravenous analgesia after PD found that patients with epidural analgesia had lower pain scores but significantly higher rates of major complications ${ }^{92}$. It has been suggested that thoracic epidural analgesia after PD is associated with hemodynamic instability, which might compromise enteric anastomoses, intestinal perfusion and 
recovery of gastrointestinal function ${ }^{92}$. In experimental acute pancreatitis and in sepsis, however, thoracic epidurals improved perfusion in gastrointestinal mucosal capillaries $^{93}$. The adverse perfusion effects of epidural analgesia appear to be related to the prolonged and extended sympathetic block. This would imply that the beneficial effects of epidural analgesia can be preserved as long as the hemodynamic consequences are adequately controlled with vasopressors ${ }^{94}$. Concerns about anastomoses have been raised after colorectal surgery, but one meta-analysis did not detect differences in rates of anastomotic leaks between patients receiving postoperative local anesthetic epidurals and those receiving systemic or epidural opioids ${ }^{95}$.

A potential drawback with epidurals is that as many as one-third of epidurals may not function satisfactorily in some centers ${ }^{96-97}$. Possible reasons may be that: catheters are not located in the epidural space; the insertion level does not cover the surgical incision; the dosage of local anesthetic and opioid are insufficient; or pump failure. For upper transverse incisions, epidural catheters should be inserted between T5 and T8 root levels. Sensory block should be tested (cold and pinprick) before induction of general anesthesia. Efforts should be made to check the sensory block on a daily (or more frequent) basis, and the infusion should be adjusted to provide sufficient analgesia to allow mobilization out of bed. It has been suggested that epidural analgesia should continue for $\geq 48 \mathrm{~h}$ and, after a successful stop-test, oral multimodal analgesia with paracetamol and non-steroidal anti-inflammatory drugs (NSAIDS)/ cyclooxygenase (COX)-2 inhibitors should be commenced together with oral opioids as required. Functioning epidural catheters may be used for a longer duration if needed. Further studies are warranted to evaluate specifically the potential risks and benefits of epidural analgesia after pancreatic surgery. The use of epidurals has not been investigated for laparoscopic pancreatic resections.

Summary and recommendation: Mid-thoracic epidurals are recommended based on data from studies on major open abdominal surgery showing superior pain relief and fewer respiratory complications compared with intravenous opioids.

Evidence level: Pain: High.

Reduced respiratory complications: Moderate.

Overall Morbidity: Low.

Recommendation grade: Weak.

\section{Intravenous analgesia}

Thoracic epidural anesthesia remains the 'gold standard' method for major open abdominal surgery, but there are situations in which it cannot be employed. Patientcontrolled analgesia (PCA) with opioids is the most common modality used as an alternative to an epidural. In a clinical trial on the implementation of a critical pathway 
for distal pancreatic surgery, PCA was the only analgesic modality used, but no comments were made on the impact of systemic analgesia on accelerating recovery ${ }^{98}$. Intravenous infusion of lidocaine has analgesic, anti- inflammatory and antihyperalgesic properties, and has been evaluated as an analgesic modality for abdominal surgery. A systematic review of 8 trials (161 patients) in which the continuous infusion of lidocaine was compared with PCA morphine for abdominal surgery, showed a decrease in the duration of ileus, LOSH, postoperative pain intensity and side effects ${ }^{99}$. A recent RCT in patients undergoing laparoscopic colorectal resection using the ERAS program showed no difference in return of gastrointestinal function and LOSH between continuous infusion of lidocaine and thoracic epidural anesthesia ${ }^{100}$.

Summary and recommendation: Some evidence supports the use of PCA or intravenous lidocaine analgesic methods. There is insufficient information on outcome after PD.

Evidence level: PCA: Very Low.

I.V. Lidocaine: Moderate.

Recommendation grade: Weak.

\section{Wound catheters and transversus abdominis plane (TAP) block}

The efficacy of wound infusion with local anesthetic agents as a postoperative analgesic method has been proven in a meta- analysis of different surgical procedures ${ }^{101}$. Conversely, a more recent meta-analysis showed that wound catheters provided no significant reduction in pain intensity (at rest or with activity) or in morphine consumption at any time after laparotomy ${ }^{102}$. No significant differences in the prevalence of infectious complications were found. These inconsistent results might be due to factors such as the type, concentration and dose of local anesthetic, type of catheter, mode of delivery, or catheter location (subcutaneous or subfascial) ${ }^{102}$. In patients undergoing colorectal surgery, a significant opioid-sparing effect and reduction of LOSH were demonstrated when local anesthetic was infused through a catheter positioned between the fascia and the peritoneum ${ }^{104}$. No significant increase in wound infections was found with the insertion of a catheter and infusion of local anesthetics. No comparison has been made with other modalities (e.g. epidural analgesia) or in enhanced recovery program.

TAP blocks anesthetize the thoracolumbar nerves (intercostal, subcostal and first lumbar), which provide sensory innervation to the anterolateral abdominal wall. The ultrasonography-guided technique for TAP blocks has been used for postoperative analgesia after abdominal surgery. A systematic analysis of 7 studies (360 patients) showed significant opioid-sparing in the post-operative period ${ }^{105}$. A meta-analysis of 5 RCTs (176 patients) confirmed previous results showing improved pain relief and reduced opioid-associated side effects ${ }^{106}$. However, no studies have compared TAP block with other analgesic methods such as epidural analgesia or infiltration of local 
anesthetic into the abdominal wound. Furthermore, no studies have used an enhanced recovery program ${ }^{107}$ and no studies have been conducted in patients undergoing pancreatic surgery.

The marked heterogeneity observed between studies included in the meta-analyses mentioned above would imply that further trials are needed to evaluate the potential use of wound catheters and TAP blocks in pancreatic surgery.

Summary and recommendation: Some evidence supports the use of wound catheters or TAP blocks in abdominal surgery. Results are conflicting and variable and mostly from studies in lower gastrointestinal surgery.

Evidence level: Wound catheters: Moderate.

TAP blocks: Moderate.

Recommendation grade: Weak.

\section{Postoperative nausea and vomiting (PONV)}

Data specifically addressing PONV after PD specifically have not been identified. One comparative (non-randomized) study $^{7}$ showed that an ERAS protocol with early mobilization, metoclopramide and removal of nasogastric tube on day 1 or day 2 decreased the rate of postoperative nausea and vomiting. Until further documentation becomes available, the suggestions for patients undergoing colorectal surgery ${ }^{3}$ should be applicable to those undergoing PD:

Patients with two risk factors (female sex, non-smoking status, history of motion sickness (or PONV) and postoperative administration of opioids ${ }^{108,109}$ ) should receive prophylaxis with dexamethasone at induction or a serotonin receptor antagonist (e.g. ondansetron, tropisetron) at the end of surgery ${ }^{110}$. High-risk individuals (three factors) should receive general anesthesia with propofol and remifentanil and no volatile anesthetics; and dexamethasone 4-8 $\mathrm{mg}$ at the beginning of surgery, supplemented with serotonin receptor antagonists or droperidol ${ }^{110}$, or 25-50 mg metoclopramide 30-60 min before the end of surgery ${ }^{111}$. Ondansetron can be used for prophylaxis and treatment. A possible risk of impaired anastomotic healing caused by single-dose dexamethasone or other steroids perioperatively has been addressed clinically and experimentally, but remains unclear ${ }^{112-115}$.

Summary and recommendation: Data from the literature on gastrointestinal surgery in patients at risk of PONV show the benefits of using different pharmacological agents depending on the patient's PONV history, type of surgery and type of anesthesia. Multimodal intervention, during and after surgery is indicated.

Evidence level: Low.

Recommendation grade: Strong. 


\section{Incision}

There are no data comparing the types of incisions for patients undergoing PD. The authors of these recommendations are comfortable with straight transverse, curved transverse and chevron incisions, indicating that all are practical. Laparoscopic resection of the pancreatic head has been reported to be feasible, ${ }^{116}$ but its future role is uncertain.

Summary and recommendation: The choice of incision is at the surgeon's discretion, and should be of a length sufficient to ensure good exposure.

Evidence level: Very low.

Recommendation grade: Strong.

\section{Avoiding hypothermia}

Several meta-analyses and RCTs have demonstrated that pre- venting inadvertent hypothermia during major abdominal surgery (such as PD) reduces the prevalence of wound infections ${ }^{117,118}$, cardiac complications ${ }^{118-120}$, bleeding and transfusion requirements $^{118-121}$, as well as the duration of post-anesthetic recovery ${ }^{122}$. Furthermore, extending systemic warming in the perioperative period $(2 \mathrm{~h}$ before and after surgery) has additional benefits ${ }^{123}$. Hence, the use of active cutaneous warming is highly recommended to reduce post-operative morbidity and enhance recovery. There is even evidence to suggest that circulating-water garments offer better temperature control than forced-air warming systems ${ }^{124-126}$.

Summary and recommendation: Intraoperative hypothermia should be avoided by using cutaneous warming, i.e. forced-air or circulating-water garment systems.

Evidence level: High.

Recommendation grade: Strong.

\section{Postoperative glycemic control}

Postoperative hyperglycaemia in patients without diabetes is a result of acquired insulin resistance. Morbidity and mortality after major abdominal surgery have been associated with increasing levels of insulin resistance ${ }^{127}$ and plasma glucose ${ }^{128}$. Such an association has also been demonstrated in pancreatic surgery. ${ }^{129}$ Data from patients subjected to colorectal surgery within an ERAS regimen indicate that higher preoperative levels of glycated hemoglobin (HBA1c) and higher postoperative levels of glucose also predict postoperative morbidity ${ }^{130}$.

Core elements of ERAS protocols attenuate postoperative insulin resistance and thus also lower glucose levels ${ }^{131,132}$. The most obvious (of several) protocols are avoidance of preoperative fasting and oral bowel preparation; use of oral carbohydrate treatment and stimulation of early resumption of gut function by optimal fluid balance and 
avoidance of systemic opioids; and the reduction of the stress response by use of epidural anesthesia.

Reducing the rate of hyperglycemia in surgical patients in intensive-care settings has been documented to reduce the rate of complications ${ }^{133,136}$. Similar trials in ward settings in patients treated with modern care regimens are wanting. The target concentration for plasma glucose is controversial ${ }^{137}$, but it seems fair to advocate that hyperglycemia should be avoided and that this will improve outcome irrespective of the baseline level. Achieving tight glycemic control with intravenous insulin is challenging in the ward setting because of the risk of hypoglycemia. Glucosuria with the risk of hypovolemia will ensue when the renal threshold is passed at $>12 \mathrm{mmol} / \mathrm{l}^{137}$. This levels been used as the control regimen in seminal trials ${ }^{133,138}$ and should probably be regarded as a limit irrespective of settings to avoid additional disturbances in fluid balance.

Summary and recommendation: Insulin resistance and hyperglycemia are strongly associated with postoperative morbidity and mortality. Treatment of hyperglycemia with intravenous insulin in the intensive-care setting improves outcomes but hypoglycemia remains a risk. Several ERAS protocol items attenuate insulin resistance and facilitate glycemic control without the risk of hypoglycemia. Hyperglycemia should be avoided as far as possible without introducing the risk of hypoglycemia.

Evidence level: Low.

Recommendation grade: Strong.

\section{Nasogastric intubation}

There is strong evidence that routine nasogastric decompression after elective laparotomy should be avoided ${ }^{139}$. Fever, atelectasis and pneumonia occur more frequently in patients with a nasogastric tube than in those without ${ }^{139,140}$. Bowel function returns earlier in patients if nasogastric decompression is avoided ${ }^{139}$. Gastroesophageal reflux is increased during laparotomy if nasogastric tubes are inserted ${ }^{141}$. The role of nasogastric tubes has not been investigated prospectively in pancreatic surgery. However, the abundant high-level evidence in other fields of abdominal surgery, including gastroduodenal surgery ${ }^{139}$, should allow for an extrapolation to patients undergoing PD and justify a 'no decompressive nasogastric tube' policy. This is also supported by some series with historic controls ${ }^{142,143}$. A large Norwegian RCT in patients after upper gastrointestinal and hepatopancreaticobiliary surgery (and including $>80$ patients who had undergone PD and were treated without routine use of a nasogastric tube) found that early oral feeding was safe and feasible ${ }^{144}$. This has also been corroborated by other non-randomized, fast-track implementation series in this field $^{5-7,9}$. In keeping with data in other areas of gastrointestinal surgery, nasogastric decompression tubes had to be replaced in $\leq 15 \%$ of patients ${ }^{6,7,9}$. Nasogastric tubes 
placed during surgery (to evacuate air) should be removed before the reversal of anesthesia. Delayed gastric emptying is a specific problem in $\approx 10-25 \%$ of patients after $P D^{6,7,9}$ and it may be necessary to insert a decompression tube in a minority of patients postoperatively.

Summary and recommendation: Pre-emptive use of nasogastric tubes postoperatively does not improve outcomes and their use is not warranted routinely. Evidence level: Moderate.

Recommendation grade: Strong.

\section{Fluid balance}

Patients undergoing abdominal surgery often receive excessive volumes of intravenous fluids during and in the days after surgery. This frequently exceeds actual fluid losses, resulting in a weight gain of 3-6 $\mathrm{kg}^{145-146}$. Excessive overload of salt and water in the perioperative period increases postoperative complication rates and delays the return of gastrointestinal function ${ }^{146-149}$. This strongly suggests that near-zero fluid balance must be achieved perioperatively. Identifying the correct amount needed is a challenge that is also complicated by the use of epidural analgesia, which causes vasodilatation and intravascular hypovolemia with hypotension, which is often interpreted and treated as fluid depletion. The result is copious volumes of fluid administration when a vasopressor would be preferable ${ }^{150}$. In a meta-analysis of elective colorectal patients, intraoperative flow-guided fluid therapy with trans- esophageal Doppler (TOD) ultrasonography to accurately assess and monitor fluid status in relation to cardiac output reduced complications and $\mathrm{LOSH}^{151}$. Other methods, such as lithium dilution (LiDCO) are evolving and may prove to be equivalent to TOD.

Hyperchloremic acidosis results from infusion of $0.9 \%$ saline. Recent studies have shown that excessive use of $0.9 \%$ saline leads to renal edema, reduced flow velocity in the renal artery, renal cortical tissue perfusion ${ }^{152}$, and an overall increase in postoperative complications when compared with balanced crystalloids ${ }^{153}$. A recent meta-analysis ${ }^{154}$ has suggested that postoperative complications and LOSH are significantly reduced if patients undergoing major abdominal surgery are maintained in fluid balance rather than fluid imbalance. The meta-analysis concluded that too much and too little fluid is detrimental to outcome. Although colloids produce better blood volume expansion and less interstitial space overload than crystalloids ${ }^{155}$, there is no evidence from clinical trials and meta-analyses that colloids result in better clinical outcome than crystalloids ${ }^{156}$. To avoid unnecessary fluid overload, vasopressors should be considered for intra- and postoperative management of epidural-induced hypotension.

Summary and recommendation: Near-zero fluid balance as well as avoiding overload of salt and water results in improved outcomes. Perioperative monitoring 
of stroke volume with trans-esophageal Doppler to optimize cardiac output with fluid boluses improves outcomes. Balanced crystalloids should be preferred to $0.9 \%$ saline.

Evidence level: Fluid balance: High.

Esophageal Doppler: Moderate.

Balanced crystalloids vs. $0.9 \%$ saline: Moderate.

Recommendation grade: Strong.

\section{Perianastomotic drains}

Perianastomotic drains are believed to ameliorate the consequences of minor leaks and allow them to be treated as controlled fistulas. One RCT comparing suction drain to no drain after pancreatic cancer resection did not show significant differences in terms of mortality or overall complication rate ${ }^{157}$. Moreover, patients who used these drains had a significantly greater incidence of intra-abdominal collections or fistulas (pancreatic and entero-cutaneous) ${ }^{157}$. A series with historic controls failed to identify any increased risk after a no-drain regimen, but this design is prone to selection bias ${ }^{158}$. Evaluation of early (post- operative day 3) versus late (postoperative day 5 and beyond) drain removal has been examined in an $\mathrm{RCT}^{159}$. Early removal of the drain in patients at low risk of pancreatic fistula (amylase value in drains $<5000 \mathrm{U} / \mathrm{l}$ at postoperative day 3 ) was associated with a significantly decreased rate of pancreatic fistula, abdominal and pulmonary complications. Until further data are available, a conservative approach with systematic postoperative drainage and early removal in patients at low risk of pancreatic fistula (firm pancreas, wide pancreatic duct ${ }^{159-161}$ ) is recommended. In accordance with this notion, it would seem wise to place a drain in patients with a soft pancreas and narrow duct, and leave this drain in situ slightly longer.

Summary and recommendation: Early drain removal after $72 \mathrm{~h}$ may be advisable in patients at low risk (i.e., amylase content in drain $<5000 \mathrm{U} / \mathrm{l}$ ) for developing a pancreatic fistula. There is insufficient evidence to recommend no routine use of drains routinely, but their use is based only on low-level evidence.

Evidence level: Early removal: High.

Recommendation grade: Early removal: Strong.

\section{Somatostatin analogues}

Somatostatin and its synthetic analogues (e.g. octreotide) reduce splanchnic blood flow and the release of pancreatic exocrine secretion ${ }^{162}$. The rationale for its use is to reduce the risk of pancreatic anastomotic fistulas by decreasing the volume of pancreatic exocrine secretions. Several RCTs have resulted in four systemic reviews and metaanalyses that assessed the possible role of a protective effect in pancreatic surgery $^{163-166}$. The most recent meta-analysis involved 17 trials with 1457 patients 
undergoing PD and 686 undergoing distal or other resections ${ }^{166}$. The authors concluded that the use of somatostatin analogues reduced the crude rate of pancreatic fistulas, but that the rate of clinically significant fistulas as well as the overall major morbidity and mortality remained unchanged ${ }^{166}$. Subgroup analyses of the PD patients showed no significant effect of somatostatin/octreotide on any of the reported outcomes ${ }^{166}$. The beneficial effect of somatostatin commonly believed to be present in cases with acknowledged risk factors (soft pancreas, small pancreatic duct) is not substantiated by the available evidence.

Summary and recommendation: Somatostatin and its analogues have no beneficial effects on outcome after PD. In general, their use is not warranted. Subgroup analyses for the variability in the texture and duct size of the pancreas are not available.

Evidence level: Moderate.

Recommendation grade: Strong.

\section{Urinary drainage}

A meta-analysis of RCTs on urinary drainage after surgery showed that suprapubic catheterization was superior to transurethral catheterization ${ }^{167}$. Patients found suprapubic catheters more acceptable, and morbidity was reduced ${ }^{167}$. Most trials in the meta-analysis evaluated urinary drainage for 4-7 days. The only trial in the metaanalysis focusing specifically on hepatopancreaticobiliary surgery ${ }^{168}$ included 82 such patients out of a total of 146 . The number of patients undergoing PD was not stated. The authors found no difference in outcomes, but argued that suprapubic catheterization is probably superior; however, the difference is likely to be small. A recent RCT with a large number of patients undergoing major surgery with thoracic epidurals found removal of transurethral catheter on postoperative day 1 to be superior in terms of infection rates and did not lead to an increased rate of recatheterization when compared with removal on day 3-5 $5^{169}$.

Summary and recommendation: Suprapubic catheterization is superior to transurethral catheterization if used for $>4$ days. Transurethral catheters can be removed safely on postoperative day 1 or 2 unless otherwise indicated.

Evidence level: High.

Recommendation grade: For suprapubic: Weak.

Transurethral catheter out postoperative day 1-2: Strong.

\section{Delayed gastric emptying (DGE)}

DGE is a specific problem after PD occurring in $\approx 10-25 \%$ of patients ${ }^{6,7,9,170}$. It may be necessary to insert a nasojejunal feeding tube in a minority of patients. DGE is as 
common after pylorus- preserving PD as after a classic Whipple's procedure ${ }^{171}$. In this context, DGE was less common in a fast-track group compared with a traditional care group in one study ${ }^{7}$. For pylorus-preserving PDs, it has been shown that constructing the duodenojejunostomy in an ante-colic (as opposed to a retro-colic) fashion results in less $D_{G E}{ }^{172}$. Occasionally, DGE persists and may necessitate enteral feeding delivered beyond the gastrojejunostomy (or even parenteral nutrition). The available definition of $\mathrm{DGE}^{170}$ is based on the assessed need for nasogastric tubes. The entity is susceptible to being over- diagnosed, and care should be taken to ensure that it does not encourage the insertion of nasogastric tubes as routine practice.

Summary and recommendation: There are no acknowledged strategies to avoid DGE. Artificial nutrition should be considered selectively in patients with DGE of long duration.

Evidence level: Very low.

Recommendation grade: Strong.

\section{Stimulation of bowel movement}

There is no high-level evidence to support a specific motility-enhancing drug. A multimodal approach involving the use of oral laxatives such as magnesium sulphate or bisacodyl may induce early gastrointestinal transit after colonic resections ${ }^{173,174}$. Some protocols for fast-track pancreatic surgery have recommended the use of laxatives postoperatively ${ }^{175}$. In a series of 255 pancreatic resections (almost 60\% PDs), oral administration of magnesium (200 mg/day) and lactulose in addition to metoclopramide on postoperative day 1 to support early start of normal bowel function was advocated $^{6}$. Along with other multimodal prescriptions, the authors concluded that this protocol was associated with a low prevalence of re-admission to hospital, mortality, and morbidity rates ${ }^{6}$. However, no randomized trial has investigated the use of oral laxatives, so further studies are necessary. As noted above, the appropriate use of epidurals and maintaining a near-zero fluid balance are associated with an enhanced return of bowel activity after abdominal surgery ${ }^{88,146}$. Chewing gum has been shown to be safe and beneficial in restoring gut activity after colorectal surgery ${ }^{176-178}$.

Summary and recommendation: A multimodal approach with epidural and nearzero fluid balance is recommended. Oral laxatives and chewing gum given postoperatively are safe and may accelerate gastrointestinal transit.

Evidence level: Laxatives: Very low.

Chewing gum: Low.

Recommendation grade: Weak. 


\section{Postoperative artificial nutrition}

Most patients tolerate normal oral intake soon after elective PD. Early oral intake in this patient group has been shown to be feasible and safe. ${ }^{6,144}$ A recent large multicenter RCT in patients undergoing only major upper gastrointestinal and hepatopancreaticobiliary surgery (including $>80$ patients undergoing PD) investigated this issue and concluded that allowing early diet is safe for these patients and that enteral tube feeding did not confer benefit ${ }^{144}$. This is in keeping with other reports ${ }^{179}$, including enteral tube feeding after other major abdominal surgery ${ }^{180}$. There are no data to support the idea that a surgeon-controlled stepwise increase from spoonfuls of water to a normal diet is safer than a patient-controlled routine as long as patients are informed about the potential of impaired gut function in the early postoperative period. Enteral or parenteral nutritional support will often be necessary if major complications develop. Parenteral nutrition is indicated only in those patients who cannot eat and drink normally, and who in addition cannot tolerate enteral nutrition ${ }^{181}$. Parenteral nutrition should be reduced as the tolerance of enteral nutritional intake increases.

Enteral tube feeding delivers artificial nutrients, but is a non-volitional intervention that bypasses the cephalic-vagal digestive reflex and carries significant risks ${ }^{182,183}$. Traditionally, benefit has been shown compared with parenteral nutrition and is based on an assumption that an early- or patient-controlled oral diet is unacceptable ${ }^{31}$. The superiority of enteral tube feeding over an early oral diet after major abdominal surgery (including after PD), has not been documented and the opposite might well be the case (as outlined above). Oral nutritional supplementation post-hospital discharge seems appealing in a patient group known to struggle to achieve dietary goals, but evidence for a benefit is lacking ${ }^{184}$.

Summary and recommendation: Patients should be allowed a normal diet after surgery without restrictions. They should be cautioned to begin carefully and increase intake according to tolerance over 3-4 days. Enteral tube feeding should be given only on specific indications and parenteral nutrition should not be employed routinely.

Evidence level: Early diet at will: Moderate.

Recommendation grade: Strong.

\section{Early and scheduled mobilization}

The relatively slow resumption of function in the stomach and gut together with significant surgical trauma leads to a prolonged recovery period in PD patients compared with many other laparotomy patients even in the absence of major complications. Extended bed rest is associated with several unwanted effects ${ }^{185,186}$. Scientific data are lacking, but the authors have observed the feasibility of written 
instructions for patients with detailed day-to-day targets postoperatively. This ensures autonomy and cooperation from patients. Daily progress can be monitored with diaries or with simple monitoring devices for patient activity. Analgesia must be adequate not only for rest, but also for early mobilization.

Summary and recommendation: Patients should be mobilized actively from the morning of the first postoperative day and encouraged to meet the daily targets for mobilization.

Evidence level: Very low.

Recommendation grade: Strong.

\section{Audit}

Systematic audit is essential to determine clinical outcome and to establish the successful implementation and continued use of a care protocol. There are also indications that audit per se improves clinical results through feedback ${ }^{187}$. It is vital to distinguish between unsuccessful implementation and lack of desired effect from an implemented protocol if results are short of the desired quality standards. Comparison with other centers using similar protocols via identical tools of registration and identical definitions of key factors is needed.

Summary and recommendation: Systematic audit improves compliance and clinical outcomes.

Evidence level: Low.

Recommendation grade: Strong.

\section{Conclusion}

ERAS $^{\circledR}$ programs have been strongly associated with reduced LOSH but this may not be the best indicator of the quality of functional recovery. An awareness of goals that improve safety and clinical outcomes is of greater importance. Emphasis must be placed on reducing morbidity with the introduction of standardized and appropriate enhanced recovery programs based on best available scientific evidence.

Multimodal ERAS programs are complex interventions that pose significant challenges to evaluation by conventional RCTs ${ }^{175,188}$. The most obvious of these challenges are standardization of the intervention and a rapidly closing window of opportunity from ethical and practical concerns ${ }^{189}$. This may, to some extent, explain the relative paucity of RCTs evaluating ERAS programs and the somewhat limited effect that has been shown on endpoints other than LOSH. In addition, interventions like these pathways are prone to show significant Hawthorne or Trial effects ${ }^{190-191}$. This implies that the collateral effect on the infra- structure and management culture to implement such a comprehensive program will have beneficial consequences in addition to those caused 
by the protocol items themselves or their synergistic effect. As has also been pointed out for this patient group ${ }^{175}$, this is nevertheless a benefit related to the use of these programs. For these reasons it may be argued that a randomized evaluation of an evidence-based ERAS protocol against traditional care may not be the way forward. Furthermore, it seems reasonable to propose that, if RCTs have proven the benefit (item by item) of two wheels, two pedals, a frame, a chain and a handle bar, then a bicycle is highly likely to be a valuable tool. Feasibility, however, must be ensured. Hence, multicenter and multinational prospective validation of a unified and comprehensive perioperative care protocol in consecutive cohorts of patients undergoing PD is warranted. 


\section{REFERENCES}

1. Varadhan KK, Neal KR, Dejong CH, Fearon KC, Ljungqvist O, Lobo DN. The enhanced recovery after surgery (ERAS) pathway for patients undergoing major elective open colorectal surgery: a meta-analysis of randomized controlled trials. Clin Nutr 2010;29:434-440.

2. Fearon KC, Ljungqvist O, Von Meyenfeldt M, Revhaug A, Dejong CH, Lassen K, Nygren J, Hausel J, Soop $\mathrm{M}$, Andersen J, Kehlet $\mathrm{H}$. Enhanced recovery after surgery: A consensus review of clinical care for patients undergoing colonic resection. Clin Nutr 2005;24:466-477.

3. Lassen K, Soop M, Nygren J, Cox PB, Hendry PO, Spies C, von Meyenfeldt MF, Fearon KC, Revhaug A, Norderval S, Ljungqvist O, Lobo DN, Dejong CH; Enhanced Recovery After Surgery (ERAS) Group. Consensus Review of Optimal Perioperative Care in Colorectal Surgery. Arch Surg 2009;144:961-969.

4. Wichmann MW, Roth M, Jauch KW, Bruns CJ. A prospective clinical feasibility study for multimodal "fast track" rehabilitation in elective pancreatic cancer surgery. Rozhl Chir 2006;85:169-175.

5. Kennedy EP, Rosato EL, Sauter PK, Rosenberg LM, Doria C, Marino IR, Chojnacki KA, Berger AC, Yeo CJ. Initiation of a critical pathway for pancreaticoduodenectomy at an academic institution--the first step in multidisciplinary team building. J Am Coll Surg 2007;204:917-923.

6. Berberat PO, Ingold H, Gulbinas A, Kleeff J, Müller MW, Gutt C, Weigand M, Friess H, Büchler MW. Fast track--different implications in pancreatic surgery. J Gastrointest Surg 2007;11:880-887.

7. Balzano G, Zerbi A, Braga M, Rocchetti S, Beneduce AA, Di Carlo V. Fast-track recovery programme after pancreatico- duodenectomy reduces delayed gastric emptying. Br J Surg 2008;95:1387-1393.

8. Montiel Casado MC, Pardo Sánchez F, Rotellar Sastre F, Martí Cruchaga P, Alvarez Cienfuegos FJ. Experience of a cephalic pancreatoduodenectomy fast-track program. Cir Esp 2010;87:378-384.

9. di Sebastiano P, Festa L, De Bonis A, Ciuffreda A, Valvano MR, Andriulli A, di Mola FF. A modified fasttrack program for pancreatic surgery: a prospective single-center experience. Langenbecks Arch Surg 2011;396:345-351.

10. Guyatt GH, Oxman AD, Vist GE, Kunz R, Falck-Ytter Y, Alonso-Coello P, Schünemann HJ; GRADE Working Group. GRADE: an emerging consensus on rating quality of evidence and strength of recommendations. BMJ 2008;336:924-926.

11. Guyatt GH, Oxman AD, Kunz R, Vist GE, Falck-Ytter Y, Schünemann HJ; GRADE Working Group. What is "quality of evidence" and why is it important to clinicians? BMJ 2008;336:995-998.

12. Guyatt GH, Oxman AD, Kunz R, Falck-Ytter $Y$, Vist GE, Liberati A, Schünemann HJ; GRADE Working Group. Going from evidence to recommendations. BMJ 2008;336:1049-1051.

13. Halaszynski TM, Juda R, Silverman DG. Optimizing postoperative outcomes with efficient preoperative assessment and management. Crit Care Med 2004;32:S76-S86.

14. Carli F, Charlebois P, Baldini G, Cachero O, Stein B. An integrated multidisciplinary approach to implementation of a fast-track program for laparoscopic colorectal surgery. Can J Anaesth 2009;56: 837-842.

15. Stergiopoulou A, Birbas K, Katostaras T, Mantas J. The effect of interactive multimedia on preoperative knowledge and postoperative recovery of patients undergoing laparoscopic cholecystectomy. Methods Inf Med 2007;46:406-409.

16. Edward GM, Naald NV, Oort FJ, de Haes HC, Biervliet JD, Hollmann MW, Preckel B. Information gain in patients using a multimedia website with tailored information on anaesthesia. Br J Anaesth 2011;106: 319-324.

17. Haines TP1, Hill AM, Hill KD, McPhail S, Oliver D, Brauer S, Hoffmann T, Beer C. Patient education to prevent falls among older hospital inpatients: a randomized controlled trial. Arch Intern Med 2011;171:516-524.

18. Clarke HD, Timm VL, Goldberg BR, Hattrup SJ. Preoperative Patient Education Reduces In-hospital Falls After Total Knee Arthroplasty. Clin Orthop Relat Res 2012;470:244-249.

19. Sewnath ME, Karsten TM, Prins MH, Rauws EJ, Obertop H, Gouma DJ. A meta-analysis on the efficacy of preoperative biliary drainage for tumors causing obstructive jaundice. Ann Surg 2002;236:17-27.

20. Saleh MM, Nørregaard P, Jørgensen HL, Andersen PK, Matzen P. Preoperative endoscopic stent placement before pancreaticoduodenectomy: a meta-analysis of the effect on morbidity and mortality. Gastrointest Endosc 2002;56:529-534. 
21. Wang Q, Gurusamy KS, Lin H, Xie X, Wang C. Preoperative biliary drainage for obstructive jaundice. Cochrane Database Syst Rev 2008;16;3:CD005444.

22. Qiu YD, Bai JL, Xu FG, Ding YT. Effect of preoperative biliary drainage on malignant obstructive jaundice: a meta-analysis. World J Gastroenterol 2011;17:391-396.

23. Garcea G, Chee W, Ong SL, Maddern GJ. Preoperative biliary drainage for distal obstruction: the case against revisited. Pancreas 2010;39:119-126.

24. van der Gaag NA, Rauws EA, van Eijck CH, Bruno MJ, van der Harst E, Kubben FJ, Gerritsen JJ, Greve JW, Gerhards MF, de Hingh IH, Klinkenbijl JH, Nio CY, de Castro SM, Busch OR, van Gulik TM, Bossuyt PM, Gouma DJ. Preoperative biliary drainage for cancer of the head of the pancreas. N Engl J Med 2010;362: 129-137.

25. Eshuis WJ, van der Gaag NA, Rauws EA, van Eijck CH, Bruno MJ, Kuipers EJ, Coene PP, Kubben FJ, Gerritsen JJ, Greve JW, Gerhards MF, de Hingh IH, Klinkenbij JH, Nio CY, de Castro SM, Busch OR, van Gulik TM, Bossuyt PM, Gouma DJ. Therapeutic delay and survival after surgery for cancer of the pancreatic head with or without preoperative biliary drainage. Ann Surg 2010;252:840-849.

26. Tonnesen H, Kehlet H. Preoperative alcoholism and postoperative morbidity. Br J Surg 1999;86: 869-874.

27. Tonnesen H, Rosenberg J, Nielsen HJ, Rasmussen V, Hauge C, Pedersen IK, Kehlet H. Effect of preoperative abstinence on poor postoperative outcome in alcohol misusers: randomised controlled trial. BMJ 1999;318:1311-1316.

28. Bluman LG, Mosca L, Newman N, Simon DG. Preoperative smoking habits and postoperative pulmonary complications. Chest 1998;113:883-889.

29. Sorensen LT, Karlsmark T, Gottrup F. Abstinence from smoking reduces incisional wound infection: a randomized controlled trial. Ann Surg 2003;238:1-5.

30. Lindström D, Sadr Azodi O, Wladis A, Tønnesen H, Linder S, Nåsell H, Ponzer S, Adami J. Effects of a perioperative smoking cessation intervention on postoperative complications: a randomized trial. Ann Surg 2008;248:739-745.

31. Goonetilleke KS, Siriwardena AK. Systematic review of peri-operative nutritional supplementation in patients undergoing pancreaticoduodenectomy. JOP 2006;7:5-13.

32. van Stijn MF, Korkic-Halilovic I, Bakker MS, van der Ploeg T, van Leeuwen PA, Houdijk AP. Preoperative Nutrition Status and Postoperative Outcome in Elderly General Surgery Patients: A Systematic Review. JPEN J Parenter Enteral Nutr. 2012;37:37-43.

33. Grotenhuis BA, Wijnhoven BP, Grüne F, van Bommel J, Tilanus HW, van Lanschot JJ. Preoperative risk assessment and prevention of complications in patients with esophageal cancer. J Surg Oncol 2010;101: 270-278.

34. Heys SD, Schofield AC, Wahle KW, Garcia-Caballero M. Nutrition and the surgical patient: triumphs and challenges. Surgeon 2005;3:139-144.

35. Cerantola $Y$, Hübner M, Grass F, Demartines N, Schäfer M. Immunonutrition in gastrointestinal surgery. Br J Surg 2011;98:37-48.

36. Marimuthu K, Varadhan KK, Ljungqvist O, Lobo DN. A meta-analysis of the effect of combinations of immune modulating nutrients on outcome in patients undergoing major open gastrointestinal surgery. Ann Surg 2012;255:1060-1068.

37. Chen B, Zhou Y, Yang P, Wan HW, Wu XT. Safety and efficacy of fish oil-enriched parenteral nutrition regimen on postoperative patients undergoing major abdominal surgery: a meta-analysis of randomized controlled trials. JPEN J Parenter Enteral Nutr 2010;34:387-394.

38. Gustafsson UO, Ljungqvist $O$. Perioperative nutritional management in digestive tract surgery. Curr Opin Clin Nutr Metab Care 2011;14:504-509.

39. Marik PE, Zaloga GP. Immunonutrition in high-risk surgical patients: a systematic review and analysis of the literature. JPEN J Parenter Enteral Nutr 2010;34:378-386.

40. Wang $Y$, Jiang ZM, Nolan MT, Jiang H, Han HR, Yu K, Li HL, Jie B, Liang XK. The impact of glutamine dipeptide-supplemented parenteral nutrition on outcomes of surgical patients: a meta-analysis of randomized clinical trials. JPEN J Parenter Enteral Nutr 2010;34:521-529.

41. Wei C, Hua J, Bin C, Klassen K. Impact of lipid emulsion containing fish oil on outcomes of surgical patients: systematic review of randomized controlled trials from Europe and Asia. Nutrition 2010;26:474-481. 
42. Sultan J, Griffin SM, Di Franco F, Kirby JA, Shenton BK, Seal CJ, Davis P, Viswanath YK, Preston SR, Hayes $N$. Randomized clinical trial of omega-3 fatty acid-supplemented enteral nutrition versus standard enteral nutrition in patients undergoing oesophagogastric cancer surgery. Br J Surg 2012;99:346-355.

43. Fujitani K, Tsujinaka T, Fujita J, et al. Prospective randomized trial of preoperative enteral immunonutrition followed by elective total gastrectomy for gastric cancer. Br J Surg 2012;99:621-629.

44. Mudge L, Isenring E, Jamieson GG. Immunonutrition in patients undergoing esophageal cancer resection. Dis Esophagus 2011;24:160-165.

45. Drover JW, Dhaliwal R, Weitzel L, Wischmeyer PE, Ochoa JB, Heyland DK. Perioperative use of argininesupplemented diets: a systematic review of the evidence. J Am Coll Surg 2011;212:385-99, 399.

46. McClave SA, Martindale RG, Vanek VW, McCarthy M, Roberts P, Taylor B, Ochoa JB, Napolitano L, Cresci G; A.S.P.E.N. Board of Directors; American College of Critical Care Medicine; Society of Critical Care Medicine. Guidelines for the Provision and Assessment of Nutrition Support Therapy in the Adult Critically III Patient: Society of Critical Care Medicine (SCCM) and American Society for Parenteral and Enteral Nutrition (A.S.P.E.N.). JPEN J Parenter Enteral Nutr 2009;33:277-316.

47. Holte K, Nielsen KG, Madsen JL, Kehlet H. Physiologic effects of bowel preparation. Dis Colon Rectum 2004;47:1397-1402.

48. Guenaga KF, Matos D, Castro AA, et al. Mechanical bowel preparation for elective colorectal surgery. Cochrane Database Syst Rev 2005;CD001544.

49. Cao F, Li J, Li F. Mechanical bowel preparation for elective colorectal surgery: updated systematic review and meta-analysis. Int J Colorectal Dis. 2012;27:803-810.

50. Lavu H, Kennedy EP, Mazo R, Stewart RJ, Greenleaf C, Grenda DR, Sauter PK, Leiby BE, Croker SP, Yeo $\mathrm{CJ}$. Preoperative mechanical bowel preparation does not offer a benefit for patients who undergo pancreaticoduodenectomy. Surgery 2010;148:278-284.

51. Ljungqvist O, Soreide E. Preoperative fasting. Br J Surg 2003;90:400-406.

52. Smith I, Kranke P, Murat I, Smith A, O'Sullivan G, Søreide E, Spies C, in't Veld B; European Society of Anaesthesiology. Perioperative fasting in adults and children: guidelines from the European Society of Anaesthesiology. Eur J Anaesthesiol 2011;28:556-569.

53. Svanfeldt M, Thorell A, Brismar K, Nygren J, Ljungqvist O. Effects of 3 days of "postoperative" low caloric feeding with or without bed rest on insulin sensitivity in healthy subjects. Clin Nutr 2003;22: 31-38.

54. Practice guidelines for preoperative fasting and the use of pharmacologic agents to reduce the risk of pulmonary aspiration: application to healthy patients undergoing elective procedures: an updated report by the American Society of Anesthesiologists Committee on Standards and Practice Parameters. Anesthesiology 2011;114:495-511.

55. Miller M, Wishart HY, Nimmo WS. Gastric contents at induction of anaesthesia. Is a 4-hour fast necessary? Br J Anaesth 1983;55:1185-1188.

56. Ljungqvist O, Nygren J, Thorell A. Modulation of post-operative insulin resistance by pre-operative carbohydrate loading. Proc Nutr Soc 2002;61:329-336.

57. Hausel J, Nygren J, Lagerkranser M, Hellström PM, Hammarqvist F, Almström C, Lindh A, Thorell A, Ljungqvist $\mathrm{O}$. A carbohydrate-rich drink reduces preoperative discomfort in elective surgery patients. Anest Analg 2001;93:1344-1350.

58. Helminen $\mathrm{H}$, Viitanen $\mathrm{H}$, Sajanti J. Effect of preoperative intravenous carbohydrate loading on preoperative discomfort in elective surgery patients. Eur J Anaesthesiol 2009;26:123-127.

59. Noblett SE, Watson DS, Huong H, Davison B, Hainsworth PJ, Horgan AF. Pre-operative oral carbohydrate loading in colorectal surgery: a randomized controlled trial. Colorectal Dis 2006;8:563-569.

60. Yuill KA, Richardson RA, Davidson HI, Garden OJ, Parks RW. The administration of an oral carbohydratecontaining fluid prior to major elective upper-gastrointestinal surgery preserves skeletal muscle mass postoperatively--a randomised clinical trial. Clin Nutr 2005;24:32-37.

61. Bisgaard T, Kristiansen VB, Hjorts $\varnothing$ NC, Jacobsen LS, Rosenberg J, Kehlet H. Randomized clinical trial comparing an oral carbohydrate beverage with placebo before laparoscopic cholecystectomy. Br J Surg 2004;91:151-158.

62. Gustafsson UO, Nygren J, Thorell A, Soop M, Hellström PM, Ljungqvist O, Hagström-Toft E. Preoperative carbohydrate loading may be used in type 2 diabetes patients. Acta Anaesthesiol Scand 2008;52:946-951. 
63. Breuer JP, von Dossow V, von Heymann C, Griesbach M, von Schickfus M, Mackh E, Hacker C, Elgeti U, Konertz W, Wernecke KD, Spies CD. Preoperative oral carbohydrate administration to ASA III-IV patients undergoing elective cardiac surgery. Anesth Analg 2006;103:1099-1108.

64. Caumo W, Levandovski R, Hidalgo MP. Preoperative anxiolytic effect of melatonin and clonidine on postoperative pain and morphine consumption in patients undergoing abdominal hysterectomy: a double-blind, randomized, placebo-controlled study. J Pain 2009;10:100-108.

65. Caumo W, Hidalgo MP, Schmidt AP, Iwamoto CW, Adamatti LC, Bergmann J, Ferreira MB. Effect of preoperative anxiolysis on postoperative pain response in patients undergoing total abdominal hysterectomy. Anaesthesia 2002;57:740-746.

66. Moiniche S, Kehlet H, Dahl JB. A qualitative and quantitative systematic review of preemptive analgesia for postoperative pain relief: the role of timing of analgesia. Anesthesiology 2002;96:725-741.

67. Walker KJ, Smith AF. Premedication for anxiety in adult day surgery. Cochrane Database Syst Rev 2009;7;4: CD002192.

68. Spyropoulos AC, Brotman DJ, Amin AN, Deitelzweig SB, Jaffer AK, McKean SC. Prevention of venous thromboembolism in the cancer surgery patient. Cleve Clin J Med 2008;75 Suppl 3:S17-26.

69. Clagett GP, Anderson FA Jr, Geerts W, Heit JA, Knudson M, Lieberman JR, Merli GJ, Wheeler HB. Prevention of venous thromboembolism. Chest 1998;114: 531S-560S.

70. Koch A, Bouges S, Ziegler S, Dinkel H, Daures JP, Victor N. Low molecular weight heparin and unfractionated heparin in thrombosis prophylaxis after major surgical intervention: update of previous meta-analyses. Br J Surg 1997;84:750-759.

71. Rasmussen MS, Jorgensen LN, Wille-Jorgensen P. Prolonged thromboprophylaxis with low molecular weight heparin for abdominal or pelvic surgery. Cochrane Database Syst Rev 2009;21;1: CD004318.

72. Horlocker TT, Wedel DJ, Benzon H, Brown DL, Enneking FK, Heit JA, Mulroy MF, Rosenquist RW, Rowlingson J, Tryba M, Yuan CS. Regional anesthesia in the anticoagulated patient: defining the risks (the second ASRA Consensus Conference on Neuraxial Anesthesia and Anticoagulation). Reg Anesth Pain Med 2003;28:172-197.

73. Horlocker TT, Wedel DJ, Rowlingson JC, Enneking FK, Kopp SL, Benzon HT, Brown DL, Heit JA, Mulroy MF, Rosenquist RW, Tryba M, Yuan CS. Regional anesthesia in the patient receiving antithrombotic or thrombolytic therapy: American Society of Regional Anesthesia and Pain Medicine Evidence-Based Guidelines (Third Edition). Reg Anesth Pain Med 2010;35:64-101.

74. Liu SS, Mulroy MF. Neuraxial anesthesia and analgesia in the presence of standard heparin. Reg Anesth Pain Med 1998;23:157-163.

75. Tryba M. European practice guidelines: thromboembolism prophylaxis and regional anesthesia. Reg Anesth Pain Med 1998;23:178-182.

76. Breivik H, Bang U, Jalonen J, Vigfússon G, Alahuhta S, Lagerkranser M. Nordic guidelines for neuraxial blocks in disturbed haemostasis from the Scandinavian Society of Anaesthesiology and Intensive Care Medicine. Acta Anaesthesiol Scand 2010;54:16-41.

77. Kakkos SK, Caprini JA, Geroulakos G, Nicolaides AN, Stansby GP, Reddy DJ. Combined intermittent pneumatic leg compression and pharmacological prophylaxis for prevention of venous thromboembolism in high-risk patients. Cochrane Database Syst Rev 2008;8;4: CD005258.

78. Lippi G, Favaloro EJ, Cervellin G. Prevention of venous thromboembolism: focus on mechanical prophylaxis. Semin Thromb Hemost 2011;37:237-251.

79. Bratzler DW, Houck PM. Antimicrobial prophylaxis for surgery: an advisory statement from the National Surgical Infection Prevention Project. Am J Surg 2005;189:395-404.

80. Nelson RL, Glenny AM, Song F. Antimicrobial prophylaxis for colorectal surgery. Cochrane Database Syst Rev 2009;21;1: CD001181.

81. Fujita S, Saito N, Yamada T, Takii Y, Kondo K, Ohue M, Ikeda E, Moriya Y. Randomized, multicenter trial of antibiotic prophylaxis in elective colorectal surgery: single dose vs 3 doses of a second-generation cephalosporin without metronidazole and oral antibiotics. Arch Surg 2007;142:657-661.

82. Steinberg JP, Braun BI, Hellinger WC, Kusek L, Bozikis MR, Bush AJ, Dellinger EP, Burke JP, Simmons B, Kritchevsky SB; Trial to Reduce Antimicrobial Prophylaxis Errors (TRAPE) Study Group. Timing of antimicrobial prophylaxis and the risk of surgical site infections: results from the Trial to Reduce Antimicrobial Prophylaxis Errors. Ann Surg 2009;250: 10-16. 
83. Darouiche RO, Wall MJ Jr, Itani KM, Otterson MF, Webb AL, Carrick MM, Miller HJ, Awad SS, Crosby CT, Mosier MC, Alsharif A, Berger DH. Chlorhexidine-Alcohol versus Povidone-lodine for Surgical-Site Antisepsis. N Engl J Med 2010;362:18-26.

84. Tschudin-Sutter S, Frei R, Egli-Gany D, Eckstein F, Valderrabano V, Dangel M, Battegay M, Widmer AF. No risk of surgical site infections from residual bacteria after disinfection with povidone-iodine-alcohol in 1014 cases: a prospective observational study. Ann Surg 2012;255:565-569.

85. Rocos B, Donaldson $\amalg$. Alcohol skin preparation causes surgical fires. Ann R Coll Surg Engl 2012;94: 87-89.

86. Block BM, Liu SS, Rowlingson AJ, Cowan AR, Cowan JA Jr, Wu CL. Efficacy of postoperative epidural analgesia: a meta-analysis. JAMA 2003;290:2455-2463.

87. Werawatganon T, Charuluxanun S. Patient controlled intravenous opioid analgesia versus continuous epidural analgesia for pain after intra-abdominal surgery. Cochrane Database Syst Rev 2005;25;1: CD004088.

88. Jorgensen H, Wetterslev J, Moiniche S, Dahl JB. Epidural local anaesthetics versus opioid-based analgesic regimens on postoperative gastrointestinal paralysis, PONV and pain after abdominal surgery. Cochrane Database Syst Rev 2000;4:CD001893.

89. Pöpping DM, Elia N, Marret E, Remy C, Tramèr MR. Protective effects of epidural analgesia on pulmonary complications after abdominal and thoracic surgery: a meta-analysis. Arch Surg 2008;143: 990-999.

90. Uchida I, Asoh T, Shirasaka C, Tsuji H. Effect of epidural analgesia on postoperative insulin resistance as evaluated by insulin clamp technique. Br J Surg 1988;75:557-562.

91. Bruns H, Rahbari NN, Löffler T, Diener MK, Seiler CM, Glanemann M, Butturini G, Schuhmacher C, Rossion I, Büchler MW, Junghans T; DISPACT Trial group. Perioperative management in distal pancreatectomy: results of a survey in 23 European participating centres of the DISPACT trial and a review of literature. Trials 2009; 10:58.

92. Pratt WB, Steinbrook RA, Maithel SK, Vanounou T, Callery MP, Vollmer CM Jr. Epidural analgesia for pancreatoduodenectomy: a critical appraisal. J Gastrointest Surg 2008;12:1207-1220.

93. Daudel F, Freise H, Westphal M, Stubbe HD, Lauer S, Bone HG, Van Aken H, Sielenkämper AW. Continuous thoracic epidural anesthesia improves gut mucosal microcirculation in rats with sepsis. Shock 2007;28:610-614.

94. Hiltebrand LB, Koepfli E, Kimberger O, Sigurdsson GH, Brandt S. Hypotension during fluid-restricted abdominal surgery: effects of norepinephrine treatment on regional and microcirculatory blood flow in the intestinal tract. Anesthesiology 2011;114:557-564.

95. Holte K, Kehlet H. Epidural analgesia and risk of anastomotic leakage. Reg Anesth Pain Med 2001;26: 111-117.

96. McLeod G, Davies H, Munnoch N, Bannister J, MacRae W. Postoperative pain relief using thoracic epidural analgesia: outstanding success and disappointing failures. Anaesthesia 2001;56:75-81.

97. Burstal R, Wegener F, Hayes C, Lantry G. Epidural analgesia: prospective audit of 1062 patients. Anaesth Intensive Care 1998;26:165-172.

98. Kennedy EP, Grenda TR, Sauter PK, Rosato EL, Chojnacki KA, Rosato FE Jr, Profeta BC, Doria C, Berger AC, Yeo CJ. Implementation of a critical pathway for distal pancreatectomy at an academic institution. $J$ Gastrointest Surg 2009;13:938-944.

99. Marret E, Rolin M, Beaussier M, Bonnet F. Meta-analysis of intravenous lidocaine and postoperative recovery after abdominal surgery. Br J Surg 2008;95:1331-1338.

100. Wongyingsinn M, Baldini G, Charlebois P, Liberman S, Stein B, Carli F. Intravenous lidocaine versus thoracic epidural analgesia: a randomized controlled trial in patients undergoing laparoscopic colorectal surgery using an enhanced recovery program. Reg Anesth Pain Med 2011;36:241-248.

101. Liu SS, Richman JM, Thirlby RC, Wu CL. Efficacy of continuous wound catheters delivering local anesthetic for postoperative analgesia: a quantitative and qualitative systematic review of randomized controlled trials. J Am Coll Surg 2006;203:914-932.

102. Gupta A, Favaios S, Perniola A, Magnuson A, Berggren L. A meta-analysis of the efficacy of wound catheters for post-operative pain management. Acta Anaesthesiol Scand 2011;55:785-796. 
103. Yndgaard S, Holst P, Bjerre-Jepsen K, Thomsen CB, Struckmann J, Mogensen T. Subcutaneously versus subfascially administered lidocaine in pain treatment after inguinal herniotomy. Anesth Analg 1994; 79:324-327.

104. Beaussier M, El'Ayoubi H, Schiffer E, Rollin M, Parc Y, Mazoit JX, Azizi L, Gervaz P, Rohr S, Biermann C, Lienhart A, Eledjam JJ. Continuous preperitoneal infusion of ropivacaine provides effective analgesia and accelerates recovery after colorectal surgery: a randomized, double-blind, placebo-controlled study. Anesthesiology 2007;107:461-468.

105. Petersen PL, Mathiesen O, Torup H, Dahl JB. The transversus abdominis plane block: a valuable option for postoperative analgesia? A topical review. Acta Anaesthesiol Scand 2010;54:529-535.

106. Siddiqui MR, Sajid MS, Uncles DR, Cheek L, Baig MK. A meta-analysis on the clinical effectiveness of transversus abdominis plane block. J Clin Anesth 2011;23:7-14.

107. Charlton S, Cyna AM, Middleton P, Griffiths JD. Perioperative transversus abdominis plane (TAP) blocks for analgesia after abdominal surgery. Cochrane Database Syst Rev 2010;8;12: CD007705.

108. Apfel CC, Kranke P, Eberhart LH, Roos A, Roewer N. Comparison of predictive models for postoperative nausea and vomiting. Br J Anaesth 2002;88:234-240.

109. Rüsch D, Eberhart L, Biedler A, Dethling J, Apfel CC. Prospective application of a simplified risk score to prevent postoperative nausea and vomiting. Can J Anaesth 2005;52:478-484.

110. Carlisle JB, Stevenson CA. Drugs for preventing postoperative nausea and vomiting. Cochrane Database Syst Rev 2006;19;3:CD004125.

111. Wallenborn J, Gelbrich G, Bulst D, Behrends K, Wallenborn H, Rohrbach A, Krause U, Kühnast T, Wiegel $M$, Olthoff $D$. Prevention of postoperative nausea and vomiting by metoclopramide combined with dexamethasone: randomised double blind multicentre trial. BMJ 2006;333:324.

112. Polat A, Nayci A, Polat G, Aksöyek S. Dexamethasone down-regulates endothelial expression of intercellular adhesion molecule and impairs the healing of bowel anastomoses. Eur J Surg 2002;168: 500-506.

113. Engelman E, Maeyens C. Effect of preoperative single-dose corticosteroid administration on postoperative morbidity following esophagectomy. J Gastrointest Surg 2010;14:788-804.

114. De Oliveira GS Jr, Almeida MD, Benzon HT, McCarthy RJ. Perioperative single dose systemic dexamethasone for postoperative pain: a meta-analysis of randomized controlled trials. Anesthesiology 2011;115:575-588.

115. Eubanks TR, Greenberg JJ, Dobrin PB, Harford FJ, Gamelli RL. The effects of different corticosteroids on the healing colon anastomosis and cecum in a rat model. Am Surg 1997;63:266-269.

116. Zureikat AH, Breaux JA, Steel JL, Hughes SJ. Can laparoscopic pancreaticoduodenectomy be safely implemented? J Gastrointest Surg 2011;15:1151-1157.

117. Kurz A, Sessler DI, Lenhardt R. Perioperative normothermia to reduce the incidence of surgical-wound infection and shorten hospitalization. Study of Wound Infection and Temperature Group. N Engl J Med 1996;334:1209-1215.

118. Scott EM, Buckland R. A systematic review of intraoperative warming to prevent postoperative complications. AORN J 2006;83:1090-13.

119. Frank SM, Fleisher LA, Breslow MJ, Higgins MS, Olson KF, Kelly S, Beattie C. Perioperative maintenance of normothermia reduces the incidence of morbid cardiac events. A randomized clinical trial. JAMA 1997;277:1127-1134.

120. Nesher N, Zisman E, Wolf T, Sharony R, Bolotin G, David M, Uretzky G, Pizov R. Strict thermoregulation attenuates myocardial injury during coronary artery bypass graft surgery as reflected by reduced levels of cardiac-specific troponin I. Anesth Analg 2003;96:328-335.

121. Rajagopalan S, Mascha E, Na J, Sessler DI. The effects of mild perioperative hypothermia on blood loss and transfusion requirement. Anesthesiology 2008;108:71-77.

122. Lenhardt R1, Marker E, Goll V, Tschernich H, Kurz A, Sessler DI, Narzt E, Lackner F. Mild intraoperative hypothermia prolongs postanesthetic recovery. Anesthesiology 1997;87:1318-1323.

123. Wong PF, Kumar S, Bohra A, Whetter D, Leaper DJ. Randomized clinical trial of perioperative systemic warming in major elective abdominal surgery. Br J Surg 2007;94:421-426.

124. Galvao CM, Liang Y, Clark AM. Effectiveness of cutaneous warming systems on temperature control: meta-analysis. J Adv Nurs 2010;66:1196-1206. 
125. Taguchi A, Ratnaraj J, Kabon B, Sharma N, Lenhardt R, Sessler DI, Kurz A. Effects of a circulating-water garment and forced-air warming on body heat content and core temperature. Anesthesiology 2004;100:1058-1064.

126. Perez-Protto S, Sessler DI, Reynolds LF, Bakri MH, Mascha E, Cywinski J, Parker B, Argalious M. Circulating-water garment or the combination of a circulating-water mattress and forced-air cover to maintain core temperature during major upper-abdominal surgery. Br J Anaesth 2010;105:466-470.

127. Sato H, Carvalho G, Sato T, Lattermann R, Matsukawa T, Schricker T. The association of preoperative glycemic control, intraoperative insulin sensitivity, and outcomes after cardiac surgery. J Clin Endocrinol Metab 2010;95:4338-4344.

128. Jackson RS, Amdur RL, White JC, Macsata RA. Hyperglycemia is associated with increased risk of morbidity and mortality after colectomy for cancer. J Am Coll Surg 2012;214:68-80.

129. Eshuis WJ, Hermanides J, van Dalen JW, van Samkar G, Busch OR, van Gulik TM, DeVries JH, Hoekstra JB, Gouma DJ. Early postoperative hyperglycemia is associated with postoperative complications after pancreatoduodenectomy. Ann Surg 2011;253:739-744.

130. Gustafsson UO, Thorell A, Soop M, Ljungqvist O, Nygren J. Haemoglobin A1c as a predictor of postoperative hyperglycaemia and complications after major colorectal surgery. Br J Surg 2009;96: 1358-1364.

131. Ljungqvist O. Insulin resistance and outcomes in surgery. J Clin Endocrinol Metab 2010;95:4217-4219.

132. Ljungqvist O. Jonathan E. Rhoads Lecture 2011: Insulin Resistance and Enhanced Recovery After Surgery. JPEN J Parenter Enteral Nutr. 2012;36:389-398.

133. van den Berghe G, Wouters P, Weekers F, Verwaest C, Bruyninckx F, Schetz M, Vlasselaers D, Ferdinande $\mathrm{P}$, Lauwers $\mathrm{P}$, Bouillon R. Intensive insulin therapy in the critically ill patients. N Engl J Med 2001;345:1359-1367.

134. Finfer S, Chittock DR, Su SY, Blair D, Foster D, Dhingra V, Bellomo R, Cook D, Dodek P, Henderson WR, Hébert PC, Heritier S, Heyland DK, McArthur C, McDonald E, Mitchell I, Myburgh JA, Norton R, Potter J, Robinson BG, Ronco JJ. Intensive versus conventional glucose control in critically ill patients. $N$ Engl J Med 2009;360:1283-1297.

135. Furnary AP, Zerr KJ, Grunkemeier GL, Starr A. Continuous intravenous insulin infusion reduces the incidence of deep sternal wound infection in diabetic patients after cardiac surgical procedures. Ann Thorac Surg 1999;67:352-360.

136. Krinsley JS. Effect of an intensive glucose management protocol on the mortality of critically ill adult patients. Mayo Clin Proc 2004;79:992-1000.

137. Van den Berghe G, Schetz M, Vlasselaers D, Hermans G, Wilmer A, Bouillon R, Mesotten D. Clinical review: Intensive insulin therapy in critically ill patients: NICE-SUGAR or Leuven blood glucose target? J Clin Endocrinol Metab 2009;94:3163-3170.

138. Van den Berghe G, Wilmer A, Hermans G, Meersseman W, Wouters PJ, Milants I, Van Wijngaerden E, Bobbaers H, Bouillon R. Intensive insulin therapy in the medical ICU. N Engl J Med 2006;354:449-461.

139. Nelson R, Edwards S, Tse B. Prophylactic nasogastric decompression after abdominal surgery. Cochrane Database Syst Rev 2007;18;3: CD004929.

140. Cheatham ML, Chapman WC, Key SP, Sawyers JL. A meta-analysis of selective versus routine nasogastric decompression after elective laparotomy. Ann Surg 1995;221:469-476.

141. Manning BJ, Winter DC, McGreal G, Kirwan WO, Redmond HP. Nasogastric intubation causes gastroesophageal reflux in patients undergoing elective laparotomy. Surgery 2001;130:788-791.

142. Fisher WE, Hodges SE, Cruz G, Artinyan A, Silberfein EJ, Ahern $\mathrm{CH}$, Jo E, Brunicardi FC. Routine nasogastric suction may be unnecessary after a pancreatic resection. HPB (Oxford) 2011;13:792-796.

143. Roland CL, Mansour JC, Schwarz RE. Routine nasogastric decompression is unnecessary after pancreatic resections. Arch Surg 2012;147:287-289.

144. Lassen K, Kjaeve J, Fetveit T, Tranø G, Sigurdsson HK, Horn A, Revhaug A. Allowing normal food at will after major upper gastrointestinal surgery does not increase morbidity: a randomized multicenter trial. Ann Surg 2008;247:721-729.

145. Tambyraja AL, Sengupta F, MacGregor AB, Bartolo DC, Fearon KC. Patterns and clinical outcomes associated with routine intravenous sodium and fluid administration after colorectal resection. World $\mathrm{J}$ Surg 2004;28: 1046-1051. 
146. Lobo DN, Bostock KA, Neal KR, Perkins AC, Rowlands BJ, Allison SP. Effect of salt and water balance on recovery of gastrointestinal function after elective colonic resection: a randomised controlled trial. Lancet 2002;359:1812-1818.

147. Lobo DN. Fluid overload and surgical outcome: another piece in the jigsaw. Ann Surg 2009;249: 186-188.

148. Chowdhury AH, Lobo DN. Fluids and gastrointestinal function. Curr Opin Clin Nutr Metab Care 2011;14: 469-476.

149. Brandstrup B, Tønnesen $H$, Beier-Holgersen R, Hjorts $\varnothing E, \emptyset$ rding $H$, Lindorff-Larsen $K$, Rasmussen MS, Lanng C, Wallin L, Iversen LH, Gramkow CS, Okholm M, Blemmer T, Svendsen PE, Rottensten HH, Thage B, Riis J, Jeppesen IS, Teilum D, Christensen AM, Graungaard B, Pott F; Danish Study Group on Perioperative Fluid Therapy. Effects of intravenous fluid restriction on postoperative complications: comparison of two perioperative fluid regimens: a randomized assessor-blinded multicenter trial. Ann Surg 2003;238:641-648.

150. Holte K, Foss NB, Svensén C, Lund C, Madsen JL, Kehlet H. Epidural anesthesia, hypotension, and changes in intravascular volume. Anesthesiology 2004;100:281-286.

151. Abbas SM, Hill AG. Systematic review of the literature for the use of oesophageal Doppler monitor for fluid replacement in major abdominal surgery. Anaesthesia 2008;63:44-51.

152. Chowdhury AH1, Cox EF, Francis ST, Lobo DN. A randomized, controlled, double-blind crossover study on the effects of 2-liters infusions of $0.9 \%$ saline and Plasma-Lyte 148 on renal blood flow velocity and renal cortical tissue perfusion in healthy volunteers. Ann Surg 2012;256:18-24.

153. Bagshaw SM, Goldstein SL, Scherer LA, Duan M, Schermer CR, Kellum JA. Major complications, mortality and resource utilization after open abdominal surgery: $0.9 \%$ saline compared to Plasma-lyte. Ann Surg 2012;255:821-829.

154. Varadhan KK, Lobo DN. A meta-analysis of randomised controlled trials of intravenous fluid therapy in major elective open abdominal surgery: getting the balance right. Proc Nutr Soc 2010;69: 488-498.

155. Lobo DN, Stanga Z, Aloysius MM, Wicks C, Nunes QM, Ingram KL, Risch L, Allison SP. Effect of volume loading with 1 liter intravenous infusions of $0.9 \%$ saline, $4 \%$ succinylated gelatine (Gelofusine) and $6 \%$ hydroxyethyl starch (Voluven) on blood volume and endocrine responses: a randomized, three-way crossover study in healthy volunteers. Crit Care Med 2010;38:464-470.

156. Senagore AJ, Emery T, Luchtefeld M, Kim D, Dujovny N, Hoedema R. Fluid management for laparoscopic colectomy: a prospective, randomized assessment of goal-directed administration of balanced salt solution or hetastarch coupled with an enhanced recovery program. Dis Colon Rectum 2009;52: 1935-1940.

157. Conlon KC, Labow D, Leung D, Smith A, Jarnagin W, Coit DG, Merchant N, Brennan MF. Prospective randomized clinical trial of the value of intraperitoneal drainage after pancreatic resection. Ann Surg 2001;234:487-493.

158. Fisher WE, Hodges SE, Silberfein EJ, Artinyan A, Ahern CH, Jo E, Brunicardi FC. Pancreatic resection without routine intraperitoneal drainage. HPB (Oxford) 2011;13:503-510.

159. Bassi C, Molinari E, Malleo G, Crippa S, Butturini G, Salvia R, Talamini G, Pederzoli P. Early versus late drain removal after standard pancreatic resections: results of a prospective randomized trial. Ann Surg 2010;252:207-214.

160. Pratt WB, Callery MP, Vollmer CM, Jr. Risk prediction for development of pancreatic fistula using the ISGPF classification scheme. World J Surg 2008;32:419-428.

161. Kawai M, Kondo S, Yamaue H, Wada K, Sano K, Motoi F, Unno M, Satoi S, Kwon AH, Hatori T, Yamamoto M, Matsumoto J, Murakami Y, Doi R, Ito M, Miyakawa S, Shinchi H, Natsugoe S, Nakagawara H, Ohta T, Takada T. Predictive risk factors for clinically relevant pancreatic fistula analyzed in 1,239 patients with pancreaticoduodenectomy: multicenter data collection as a project study of pancreatic surgery by the Japanese Society of Hepato-Biliary-Pancreatic Surgery. J Hepatobiliary Pancreat Sci 2011;18:601-608.

162. Suc B, Msika S, Piccinini M, Fourtanier G, Hay JM, Flamant Y, Fingerhut A, Fagniez PL, Chipponi J; French Associations for Surgical Research. Octreotide in the prevention of intra-abdominal complications following elective pancreatic resection: a prospective, multicenter randomized controlled trial. Arch Surg 2004;139:288-294. 
163. Li-Ling J, Irving M. Somatostatin and octreotide in the prevention of postoperative pancreatic complications and the treatment of enterocutaneous pancreatic fistulas: a systematic review of randomized controlled trials. Br J Surg 2001;88:190-199.

164. Connor S, Alexakis N, Garden OJ, Leandros E, Bramis J, Wigmore SJ. Meta-analysis of the value of somatostatin and its analogues in reducing complications associated with pancreatic surgery. Br J Surg 2005;92:1059-1067.

165. Alghamdi AA, Jawas AM, Hart RS. Use of octreotide for the prevention of pancreatic fistula after elective pancreatic surgery: a systematic review and meta-analysis. Can I Surg 2007;50:459-466.

166. Koti RS, Gurusamy KS, Fusai G, Davidson BR. Meta-analysis of randomized controlled trials on the effectiveness of somatostatin analogues for pancreatic surgery: a Cochrane review. HPB (Oxford) 2010; 12:155-165.

167. McPhail MJ, Abu-Hilal M, Johnson CD. A meta-analysis comparing suprapubic and transurethral catheterization for bladder drainage after abdominal surgery. Br J Surg 2006;93:1038-1044.

168. Baan AH, Vermeulen H, van der Meulen J, Bossuyt P, Olszyna D, Gouma DJ. The effect of suprapubic catheterization versus transurethral catheterization after abdominal surgery on urinary tract infection: a randomized controlled trial. Dig Surg 2003;20:290-295.

169. Zaouter C, Kaneva P, Carli F. Less urinary tract infection by earlier removal of bladder catheter in surgical patients receiving thoracic epidural analgesia. Reg Anesth Pain Med 2009;34:542-548.

170. Wente MN, Bassi C, Dervenis C, Fingerhut A, Gouma DJ, Izbicki JR, Neoptolemos JP, Padbury RT, Sarr MG, Traverso LW, Yeo CJ, Büchler MW. Delayed gastric emptying (DGE) after pancreatic surgery: a suggested definition by the International Study Group of Pancreatic Surgery (ISGPS). Surgery 2007;142: 761-768.

171. Diener MK, Fitzmaurice C, Schwarzer G, Seiler CM, Antes G, Knaebel HP, Büchler MW. Pyloruspreserving pancreaticoduodenectomy (pp Whipple) versus pancreaticoduodenectomy (classic Whipple) for surgical treatment of periampullary and pancreatic carcinoma. Cochrane Database Syst Rev 2011;11;5:CD006053.

172. Tani M, Terasawa H, Kawai M, Ina S, Hirono S, Uchiyama K, Yamaue H. Improvement of delayed gastric emptying in pylorus-preserving pancreaticoduodenectomy: results of a prospective, randomized, controlled trial. Ann Surg 2006;243: 316-320.

173. Basse L, Madsen JL, Kehlet $\mathrm{H}$. Normal gastrointestinal transit after colonic resection using epidural analgesia, enforced oral nutrition and laxative. Br J Surg 2001;88:1498-1500.

174. Zingg U, Miskovic D, Pasternak I, Meyer P, Hamel CT, Metzger U. Effect of bisacodyl on postoperative bowel motility in elective colorectal surgery: a prospective, randomized trial. Int J Colorectal Dis 2008; 23:1175-1183.

175. Ypsilantis E, Praseedom RK. Current status of fast-track recovery pathways in pancreatic surgery. JOP 2009;10:646-650.

176. Noble EJ, Harris R, Hosie KB, Thomas S, Lewis SJ. Gum chewing reduces postoperative ileus? A systematic review and meta-analysis. Int I Surg 2009;7:100-105.

177. Vasquez W, Hernandez AV, Garcia-Sabrido JL. Is gum chewing useful for ileus after elective colorectal surgery? A systematic review and meta-analysis of randomized clinical trials. J Gastrointest Surg 2009; 13:649-656.

178. de Castro SM, van den Esschert JW, van Heek NT, Dalhuisen S, Koelemay MJ, Busch OR, Gouma DJ. A systematic review of the efficacy of gum chewing for the amelioration of postoperative ileus. Dig Surg 2008;25:39-45.

179. Yermilov I, Jain S, Sekeris E, Bentrem DJ, Hines OJ, Reber HA, Ko CY, Tomlinson JS. Utilization of parenteral nutrition following pancreaticoduodenectomy: is routine jejunostomy tube placement warranted? Dig Dis Sci 2009;54: 1582-1588.

180. Koretz RL. Enteral nutrition: a hard look at some soft evidence. Nutr Clin Pract 2009;24:316-324.

181. Gianotti L, Meier R, Lobo DN, Bassi C, Dejong CH, Ockenga J, Irtun O, MacFie J; ESPEN. ESPEN Guidelines on Parenteral Nutrition: pancreas. Clin Nutr 2009;28:428-435.

182. Han-Geurts IJ, Verhoef C, Tilanus HW. Relaparotomy following complications of feeding jejunostomy in esophageal surgery. Dig Surg 2004;21:192-196. 
183. Lobo DN, Williams RN, Welch NT, Aloysius MM, Nunes QM, Padmanabhan J, Crowe JR, Iftikhar SY, Parsons SL, Neal KR, Allison SP, Rowlands BJ. Early postoperative jejunostomy feeding with an immune modulating diet in patients undergoing resectional surgery for upper gastrointestinal cancer: a prospective, randomized, controlled, double-blind study. Clin Nutr 2006;25:716-726.

184. Lidder PG, Lewis S, Duxbury M, Thomas S. Systematic review of postdischarge oral nutritional supplementation in patients undergoing GI surgery. Nutr Clin Pract 2009;24:388-394.

185. Kehlet H, Wilmore DW. Multimodal strategies to improve surgical outcome. Am J Surg 2202;183: 630-641.

186. Convertino VA. Cardiovascular consequences of bed rest: effect on maximal oxygen uptake. Med Sci Sports Exerc 1997;29:191-196.

187. Jamtvedt G, Young JM, Kristoffersen DT, O'Brien MA, Oxman AD. Audit and feedback: effects on professional practice and health care outcomes. Cochrane Database Syst Rev 2006;19;CD000259.

188. Campbell M, Fitzpatrick R, Haines A, Kinmonth AL, Sandercock P, Spiegelhalter D, Tyrer P. Framework for design and evaluation of complex interventions to improve health. BMJ 2000;321:694-696.

189. Lassen K, Høye A, Myrmel T. Randomised trials in surgery: the burden of evidence. Rev Recent Clin Trials 2012;7:244e8.

190. Franke RH, Kaul JD. The Hawthorne experiments: first statistical interpretation. Am Soc Rev 1978;43: 623-643.

191. McCarney R, Warner J, Iliffe S, van Haselen R, Griffin M, Fisher P. The Hawthorne Effect: a randomised, controlled trial. BMC Med Res Methodol 2007;7:30. 


\section{CHAPTER}

Study protocol PANDA trial:

PAncreaticoduodeNectomy and Drainage After

surgery: Multicenter randomized controlled trial of

prophylactic abdominal drain versus no drain policy after pancreaticoduodenectomy, within an enhanced recovery after surgery pathway

MME Coolsen, $\mathrm{V}$ van Woerden, RM van Dam, G van Breukelen, K Lassen, MG Besselink, CHC Dejong 


\section{ABSTRACT}

\section{Background}

Prophylactic abdominal drainage after pancreaticoduodenectomy has been standard care for many years. However, recent studies assessing the efficacy of prophylactic abdominal drains suggest these drains have no advantage over avoiding prophylactic postoperative drainage. In fact, some studies show that abdominal drains may even be harmful in terms of an increased risk of infection. This study was designed to assess the feasibility and safety of omitting prophylactic abdominal drainage after pancreaticoduodenectomy.

\section{Methods and design}

Trail design: A multicenter, randomized controlled trial with stratification for center, and two treatment arms: prophylactic abdominal postoperative drainage versus a no drain policy within an enhanced recovery after surgery pathway.

Participants: The patients included in this study are patients above the age of 18 , who require elective pancreaticoduodenectomy, such as a Pylorus Preserving PancreaticoDuodenectomy (PPPD) or a classical Whipple's procedure, who have an ASA score of I through III and are able to give informed consent. Patients undergoing double bypass surgery are analyzed as a separate group. Intervention: Omitting prophylactic postoperative abdominal drain.

Comparison: Prophylactic abdominal postoperative drain versus a no drain policy within an Enhanced Recovery After Surgery pathway.

Outcome: The primary outcome is the need for (re-)interventions, which are defined as drain related complications. Re-interventions are defined as the administration of antibiotics, endovascular coiling or stenting, ultrasound or CT guided drainage or relaparotomy. Secondary outcomes of interest are overall complications, a composite endpoint for pancreaticoduodenectomy, functional recovery and delay in discharge, readmissions, mortality and total length of hospital stay.

\section{Discussion}

This randomized controlled trial will shed light on whether prophylactic drainage after pancreaticoduodenectomy can be abandoned. 


\section{INTRODUCTION}

Pancreaticoduodenectomy (PD) is a surgical procedure with morbidity rates nowadays varying from 30 to $65 \%$, with leakage of the pancreaticojejunostomy being the most feared complication. Recent advances in surgical techniques have decreased the mortality rate for pancreatic surgery to less than $5 \%{ }^{1}$.

Intra-abdominal prophylactic drainage after surgery has been standard clinical practice for many decades. Many surgeons believe that postoperative drainage allows for the early detection of anastomotic leakage of the pancreaticojenunostomy, gastrojejunostomy or hepatojejunostomy as well as postoperative hemorrhage and that it may prevent fluid collections ${ }^{2-4,6,10}$. However, recent studies assessing the efficacy of postoperative drains in abdominal surgery show that placement of a postoperative drain might not be advantageous over omitting a postoperative drain ${ }^{3-6}$. Kawai et al. already established that short term post-operative drainage in pancreatic surgery has favorable effects over long term drainage ${ }^{6}$. Other studies suggest that routine placement of drains after pancreatic surgery is unnecessary and may be harmful to the patient ${ }^{3,5}$. There is sufficient evidence that prophylactic abdominal postoperative drains do not always drain fluid or hemorrhagic collections. It is therefore questionable whether drainage is useful if fluids are not adequately drained and can still become infected ${ }^{2,3}$. In several studies the feasibility of early drain removal was assessed, and these studies suggested early drain removal did not result in an increase in mortality or morbidity but actually reduced the rate of pancreatic fistula and intraabdominal infections ${ }^{6,8,9,11}$.

Postoperative drains are uncomfortable, can cause pain and interfere with mobilization and therefore may delay recovery. An Enhanced Recovery After Surgery (ERAS) pathway aims for an accelerated recovery in which early mobilization is one of the main items $^{8,9,11}$.

Information about prophylactic postoperative drainage after pancreatic resection remains sparse and controversial. This study was designed to gather conclusive information regarding the use of postoperative prophylactic abdominal drainage after PD.

\section{METHODS/DESIGN}

\section{Study Design}

The PANDA-Trial is a multicenter, international, randomized controlled trial, conducted after elective PD in adult patients for a pancreatic neoplasm. Prophylactic postoperative drainage versus a no drain policy within an enhanced recovery after 
surgery pathway will be assessed. Within each center, patients will be randomly allocated to receive a prophylactic abdominal drain after surgery versus no drain.

\section{Objective}

The objective of this study is to assess the feasibility and safety of omitting prophylactic abdominal postoperative drainage after pancreatic surgery.

\section{Outcome}

\section{Primary study parameter}

Primary outcome: rate of patients undergoing radiologic and/or surgical complications. All radiological and/or surgical interventions (Dindo $3 a$ and above) for complications are documented and categorized (Table 7.1). Interventions are defined as image guided percutaneous drainage, endovascular coiling or stenting, or relaparotomy.

Table 7.1 Dindo-Clavien classification

\begin{tabular}{|c|c|}
\hline Grade & Definition \\
\hline Grade I & $\begin{array}{l}\text { Any deviation from the normal post-operative course, without the need for } \\
\text { intervention of any kind. Allowed therapeutic regimens are: anti-emetics, } \\
\text { antipyretics, analgesics, diuretics, electrolytes and physiotherapy. This also } \\
\text { includes wound infections treated at bedside. }\end{array}$ \\
\hline Grade II & $\begin{array}{l}\text { Requiring pharmacological treatment with drugs other than described for } \\
\text { Grade I. This includes blood transfusions and total parenteral nutrition. }\end{array}$ \\
\hline Grade III & Requiring surgical, endoscopic or radiological intervention \\
\hline Grade IIla & Intervention not under general anesthesia. \\
\hline Grade IIIb & Intervention under general anesthesia. \\
\hline Grade IV & $\begin{array}{l}\text { Life-threatening complication requiring ICU management (including CNS } \\
\text { complications)* }\end{array}$ \\
\hline Grade IVa & Single organ dysfunction (including dialysis) \\
\hline Grade IVb & Multi organ dysfunction \\
\hline Grade V & Death of a patient \\
\hline Suffix "d" (disability) & $\begin{array}{l}\text { This is added if the patient still suffers from the complication at the time of } \\
\text { discharge }\end{array}$ \\
\hline
\end{tabular}

*Brain hemorrhage, ischemic stroke, subarachnoidal bleeding, but excluding transient ischemic attacks. CNS, central nervous system; IC, intermediate care; ICU, intensive care unit.

\section{Secondary study parameters}

1) Overall post-operative complications

Complication rates in both groups are documented, analyzed and compared.

2) Composite Endpoint for Pancreaticoduodenectomy ${ }^{12}$

Also, the incidence of the following composite endpoint is reported and analyzed.

The composite endpoint consists of:

- Intra-abdominal abscess 
- $\quad$ Sepsis

- Gastro-jejunostomy leakage

- Hepatojejunostomy/bile leakage

- Pancreatic Fistula (pancreaticojejunostomy-, pancreaticogastrostomy leakage)

- Post-pancreatic surgery hemorrhage

- $\quad$ Delayed Gastric Emptying

- 90 day- mortality

3) Functional recovery

Functional recovery is considered a secondary outcome of the study and is registered in order to determine whether or not there is a delay in discharge.

Therefore recovery criteria are defined as follows:

- Adequate pain control with oral analgesia only

- Tolerance of solid food

- No intravenous fluids

- Independently mobile or same level as prior to admission

The rates of functional recovery and the cause of delay of discharge will be analyzed and compared between the drainage and no-drainage group. Functional recovery criteria are equal to discharge criteria. In practice, patients often meet recovery criteria but are not discharged from the hospital. Reasons for this delay in discharge after recovery can be due to practical or logistical problems.

4) Discharge criteria

When functional recovery criteria have been met, the patient can be discharged if he or she is willing to go home. Therefore discharge criteria are defined as follows: adequate pain control with oral analgesia only, tolerance of solid food, no intravenous fluids, independently mobile or same level as prior to admission, all of the above and willing to go home.

5) Length of Hospital Stay (LOS)

To assess whether discontinuing postoperative abdominal drainage has an effect on postoperative length of hospital stay the difference in hospital stay between both groups will be assessed. LOS will be measured as days after surgery, with the day of operation as day 0 . Next to delay in discharge and its causes, the total length of hospital stay is documented.

6) Mortality

Pancreatic surgery is commonly known for its high morbidity and mortality rates. Death is the most serious complication of pancreatic resections and in order to continuously assess the safety of the study this also is an outcome of interest and is considered a secondary outcome of the study. Causes of death will be analyzed to check for associations between death and surgery, drainage or disease. Mortality will be scored after 90 days of operation. 
7) Readmissions

Readmission in this study is defined as re-hospitalization as a consequence of a complication of the primary operation. Readmission can be an important indication of complications or a result of early discharge and is therefore considered a secondary outcome of this study. The reason for readmission and the Dindoclassification of the cause of readmission will be analyzed.

Readmission for recurrent or progressive disease is not defined as a readmission. Abdominal wall herniation is a long-term complication and is not considered to be an endpoint of this study.

\section{Hypothesis}

Drains are typically placed as result of long standing practice without robust evidence. An abdominal drain does not always contribute to the patient's health and sometimes may even be harmful. We hypothesized that avoidance of prophylactic abdominal drainage after pancreaticoduodenectomy leads to fewer secondary interventions, fewer complications and a reduction in length of hospital stay.

\section{Setting}

The PANDA-Trial will be conducted as a multicenter international randomized controlled trial.

\section{Inclusion}

The baseline demographics for patients participating in the PANDA-trial are outlined in Table 7.2. The patients included in this study are patients above the age of 18 years who require elective PD such as PPPD and Whipple's procedure. Patients planned for undergoing PD but who turn out to receive a double bypass or no operation (e.g. in case of irresectability of the process) will be considered a subgroup within this study and will be analyzed separately.

For inclusion, all of the following inclusion criteria must be met: 1) the patient is diagnosed with a pancreas head tumor, 2) the patient will undergo elective PD, 3) the patient's age is more than 18 years, 4) the patient has ASA I, II, or III status 5) the patient has signed a written informed consent.

Exclusion criteria are: 1) patients undergoing emergency surgery, 2) patients with an ASA IV or V status, 3) patients who are pregnant, or 4) the impossibility to obtain informed consent. 
Table 7.2 Baseline demographics for patients participating in the PANDA-trial

\begin{tabular}{lll}
\hline Age & Years & \\
Gender & Male / Female & \\
Classification tumor & 1. Pancreas head Neoplasm & 4. Chronic pancreatitis \\
& 2. Duodenum Neoplasm & 5. Peri-ampullairy neoplasm \\
3. Ampullary Neoplasm & 6. Other \\
Co-morbidity & 0. None & 4. Portal hypertension \\
& 1. Cardiovascular diseases & 5. Cirrhosis \\
& 2. Pulmonary diseases & 6. Recent Alcohol abuse \\
Body Mass Index & 3. Diabetes Mellitus & 7. Other \\
Smoking & Weight (kg) / Height ${ }^{2}$ (m) & \\
Social environment & Yes / No & \\
& 1. Single / Widow(er) & 3. Single, with Children \\
Actual performed surgery & 2. Cohabitation / Married & 4. Cohabitation / Married with children \\
& 1. Whipple & 3. Double Bypass \\
Duration of operation & 2. PPPD & 4. Other \\
ASA-classification & Minutes & \\
Epidural & I/II / III & \\
Pre-operative Antibiotics & Yes / No & \\
Functional drain & Yes / No & \\
\hline & Yes / No &
\end{tabular}

\section{Number of participants}

A RCT by Conlon et al. ${ }^{2}$, randomizing between a drain or no drain, showed a reduction in radiologic or surgical interventions from about $21.5 \%$ to $12 \%$ in the no drain group. Our current intervention percentage is $25 \%$. The sample size calculations were based on the premise of reducing the percentage of patients needing intervention from $25 \%$ to $12.5 \%$. With a alpha set at 0.05 (two sided) and a $\beta$ of 0.2 yielding an $80 \%$ power, data of 306 patients undergoing a Whipple or PPPD procedure need to be analyzed. Assuming there is a $10 \%$ withdrawal rate, a total of 340 patients need to be included in this study. Adjustment for center, by treatment effects and prognostic factors necessitates a further increase to 360 patients, which implies 180 per treatment arm.

\section{Randomization procedure}

Within each center, patients will be randomized in blocks of size 10 by equal allocation, meaning each participant has an equal likelihood to will be randomized in the drain or no drain group. Computerized randomization will be pre-stratified on center and in blocks of size 10 to ensure balanced allocation in every center. The patients will be divided into two groups: a control group receiving a prophylactic abdominal drain after PD (group A), and group receiving no drain (group B).

Computerized randomization will take place after the operation before closing the abdomen. If patients are randomized to receive a drain, the drain will be placed by the surgeon during the operation (PD) directly before closure of the abdomen. The drain will be removed at postoperative day 4 . 


\section{Blinding}

The study group will not receive a placebo drain of any kind because the effect of increased mobility on hospital stay will also be assessed, and because a placebo drain might, just like a real drain, increase the risk of infections and thereby reduce the contrast between both treatment arms with respect to the primary outcome, i.e. the need for (re-)intervention. Blinding of physicians or patients was not an option in this study. The possibility of adding a 'placebo' drain by attaching a drain to the abdominal wall, filling the container with a saline solution and covering up the container by a nontransparent plastic bag was considered. However, this was abandoned on practical and ethical grounds. As a placebo drain was not feasible, outcome assessment could not be blinded.

\section{Interventions}

\section{Investigational treatment}

Within each center, patients in this study will be randomized in a drain or a no-drain group (see flowchart) in blocks of size 10. The patients in the drain group constitute the control group, which will receive 1 or 2 drains according to routinely applied protocols. The no-drain group represents the study group and will undergo the exact same operation as the drain group. The only difference is that these patients will not receive a prophylactic postoperative abdominal drain.

Both the control group and the study group will undergo treatment within an Enhanced Recovery After Surgery program. The ERAS program especially designed for pancreatic surgery is shown in appendix 3 and 4 . The program includes; clear liquids up to 2 hours before surgery, no pre-anesthetic medication, preoperative antibiotics, preoperative heparin, epidural anesthesia, no nasogastric drainage or removal of the nasogastric drainage directly after surgery, food at will postoperatively, early mobilization postoperatively, avoidance of long-term acting anesthetics, normothermia during surgery and adequate pain control ${ }^{13}$.

Patients are asked to keep a postoperative journal until their discharge in which can be described whether certain postoperative recovery goals are met (e.g. out of bed for 2 hours, drinking 0.5 I of water), also patients are asked to point out on a scale how much pain they experience and if they feel nauseous. Patients will not receive additional monitoring as part of the PANDA-Trial. They will be monitored on the recovery and the surgical ward twice daily according to normal healthcare standards.

\section{Additional drainage}

When additional drainage is required, irrespective of the cause or setting of the requirement, the drain should be placed as described by routinely applied protocols. Additional drainage is considered an intervention and is recorded accordingly. 


\section{Follow-up}

Patients will not receive a separate follow up for this study. Readmissions and mortality are actively checked after 30,60 and 90 days from discharge of each patient. They are registered if and when they occur within 90 days from surgery either in hospital or after discharge from the hospital or when readmission is a consequence of the pancreatic surgery.

\section{Monitoring}

All adverse events are reported and assessed by a Data Safety Monitoring Board and in case of serious adverse events such as death, this will be reported to the Medical Research Ethics Committee (MREC) as well. The investigator will inform the patients if serious adverse events occur, on the basis of which it appears that the disadvantages of participation may be significantly greater than was foreseen in the research proposal. This also applies if the control group experiences more adverse effects than the study group. The study will be suspended pending further review by the accredited MREC. The investigator is in charge of keeping all patients informed.

\section{Statistical analysis}

\section{Descriptive statistics}

1) Baseline characteristics

The baseline characteristics of the participating patients will be summarized with descriptive statistics per treatment arm and overall in a table. Quantitative characteristics are summarized by their mean and standard deviation, categorical characteristics are summarized by percentages.

2) Interventions and complications

Interventions and complications are presented as frequencies per treatment arm per center and per treatment arm in total, using patients as units of analysis. Severity of interventions of complications (DINDO-classification) and the complications will likewise be summarized. Complications will also be registered as a Composite Endpoint, showing drain related complications as a positive outcome. Drain related complications are defined as; anastomotic leakage (defined according to International Study Group of Pancreatic Fistula, ISGPF) ${ }^{14}$, intra-abdominal collections and intra-abdominal hemorrhage (defined according to the International Study Group of Pancreatic Surgery, ISGPS classification) ${ }^{15}$.

3) Readmissions

The number of readmissions (shown in percentage of the population), the cause of readmission and interventions are registered. 
4) Length of hospital stay

The length of hospital stay is measured in days and shown in mean and median number of days with standard deviation, per center and for the total sample. Length of hospital stay is defined as, days/nights in hospital, the day of operation being day 0 .

5) Mortality

Mortality is defined as death within 90 days of surgery related to the surgery. Death due to recurrent or progressive disease is not defined as mortality in this study. Mortality is displayed as percentage of the study population, per center and for the total sample.

6) Functional recovery

Functional recovery is registered and analyzed to identify causes of delay in discharge. Furthermore, the number of days the patient resides in the hospital despite functional recovery is an outcome of interest.

7) Amylase concentration in drain fluids

Amylase can be measured in patients with a functional drain when one suspects the presence of an anastomotic leakage. Amylase is measured in Units / Liter and will be presented as mean value with standard deviation.

\section{Inferential statistics}

The primary efficacy analysis will be an intention-to-treat analysis, i.e. with patients grouped by treatment assignment (irrespective of actual treatment). The secondary analysis will be with grouping by actual treatment or "per protocol" (irrespective of assigned treatment). We note that this secondary analysis may suffer from hidden confounders, depending on the causes of, and reasons for, protocol deviation.

To correct for such confounding as much as possible, the reason for protocol deviation (e.g. a complication during surgery) will be documented, and information on the occurrence of such reasons/complications will be recorded for all patients in both arms to allow statistical corrections afterwards.

An interim analysis will be performed after $50 \%$ of the planned number of patients has been included, that is, after the first 180 patients. This analysis concerns efficacy as indicated by the primary outcome, and safety as indicated by mortality. The following stopping rules will be used:

Efficacy-stop: stop the trial if the primary outcome difference between both treatment arms (drain and no drain) is significant at $\alpha=0.005$ two-tailed, following the O'Brien and Fleming (1979) cut-off for a single interim analysis halfway a trial with a final $\alpha=0.05$ two-tailed. The significance test at interim has a power of $44 \%$ to detect an outcome difference of $25 \%$ with drain against $10 \%$ without drain, and a power of $71 \%$ for a difference of $25 \%$ versus $5 \%$. The power at the final analysis is as described in the section with the sample size calculation. 
Safety-stop: stop if at least one of the following two criteria is met:

1) Mortality is significantly higher in the no-drain arm (new treatment) than in the drain arm (standard treatment), using a one-tailed test with $\alpha=0.10$. This test has a power of $50 \%$ to detect a difference in mortality of $5 \%$ with drain against $10 \%$ without drain.

2) Mortality in the no-drain arm is significantly higher than $5 \%$, following a one-tailed test with $\alpha=0.10$. The trial will thus stop if mortality in the no-drain arm equals or exceeds 8 of 90 patients (this applies with and without continuity correction). This test has a power of $82 \%$ to detect a true mortality of $10 \%$ in the no-drain arm.

Dichotomous outcomes will be analyzed with logistic regression, with a predictor's treatment, center, and possibly prognostic baseline variables like age, sex, general health status to increase power and precision for the treatment effect of interest. Continuous outcomes such as length of hospital stay will be analyzed with linear regression, possibly after data transformation in case of severe non-normality or outliers. The primary and secondary outcomes are univariate without correction for confounders, but with the pre-stratification variable center as covariate. Other prognostic variables are subsequently included to increase power for the test of the outcome difference between arms, and to increase precision of the effect estimate, by reducing unexplained variance. These other variables will be dropped from the model one-by-one if not significant at $\alpha=0.05$ two-tailed. Outcome results per center will be presented if treatment by center interaction is found.

\section{Consent and ethical considerations}

\section{Regulation statement}

This study will be conducted according to the principles of the Declaration of Helsinki (October 2008) and with the Medical Research Involving Human Subjects Act.

\section{Recruitment and consent}

The surgeon sees patients eligible for PD in the out-patient clinic. He will inform the patients about the study from the moment patients are going to be planned for operation and will provide patients with written study information. Patients will be able to read the information about the study at home and will be given two weeks to reflect on their participation in the study. The investigator will contact the patient after 2 weeks to assess whether the patient is willing to participate. If a patient is willing to participate a written informed consent will be obtained by the investigator.

\section{Individual withdrawal}

Individuals can leave the study at any time without explanation and without any consequences. The investigator can decide to withdraw an individual from the study for 
urgent medical reasons. If the operating physician decides to defer from the randomization this is documented and analyzed using an intention to treat analysis.

\section{DISCUSSION}

The use of surgically placed prophylactic abdominal drains after pancreaticoduodenectomy is still considered as routine in daily practice. The fear of major complications following a pancreatic leak or hemorrhage which might go unnoticed without a drain keeps surgeons from omitting abdominal drainage after surgery. Some surgeons have adopted an early drain removal policy to avoid the late drain-associated complications but to take advantage of the beneficial effects as a detector of early postoperative problems. However, some important leaks become only apparent later in the postoperative course, which would be after the early removal of a drain. Evidence for omitting drainage after pancreaticoduodenectomy currently presented is sparse and of low level. To change practice high level evidence needs to be gathered, hence a randomized controlled trials need to be conducted. With this multicenter international $\mathrm{RCT}$ in which we randomize between prophylactic drainage or no prophylactic drainage after pancreaticoduodenectomy with patients managed in an enhanced recovery after surgery pathway we hope to shed light on the risk and benefits of omitting a postoperative intra-abdominal drain. 


\section{REFERENCES}

1. de Wilde RF, Besselink MG, van der Tweel I, de Hingh IH, van Eijck CH, Dejong CH, Porte RJ, Gouma DJ, Busch OR, Molenaar IQ; Dutch Pancreatic Cancer Group. Impact of nationwide centralization of pancreaticoduodenectomy on hospital mortality. Br J Surg 2012;99:404-410.

2. Petrowsky H, Demartines N, Rousson V, Clavien PA. Evidence-based value of prophylactic drainage in gastrointestinal surgery: a systematic review and meta-analyses. Ann Surg, 2004;240:1074-1084.

3. Conlon KC, Labow D, Leung D, Smith A, Jarnagin W, Coit DG, Merchant N, Brennan MF. Prospective randomized clinical trial of the value of intraperitoneal drainage after pancreatic resection. Ann Surg, 2001;234:487-493.

4. Buchler MW, Friess $\mathrm{H}$. Evidence forward, drainage on retreat: still we ignore and drain? Ann Surg. 2006;244:8-9.

5. Heslin MJ, Harrison LE, Brooks AD, Hochwald SN, Coit DG, Brennan MF. Is intra-abdominal drainage necessary after pancreaticoduodenectomy? J Gastrointest Surg. 1998;2:373-378.

6. Kawai M, Tani M, Terasawa H, Ina S, Hirono S, Nishioka R, Miyazawa M, Uchiyama K, Yamaue H. Early removal of prophylactic drains reduces the risk of intra-abdominal infections in patients with pancreatic head resection: prospective study for 104 consecutive patients. Ann Surg, 2006;244:1-7.

7. Shinchi H, Wada K, TraversoLW. The usefulness of drain data to identify a clinically relevant pancreatic anastomotic leak after pancreaticoduodenectomy? J Gastrointest Surg. 2006;10:490-498.

8. Berberat PO, Ingold H, Gulbinas A, Kleeff J, Müller MW, Gutt C, Weigand M, Friess H, Büchler MW. Fast track--different implications in pancreatic surgery. J Gastrointest Surg. 2007;11:880-887.

9. Balzano G, Zerbi A, Braga M, Rocchetti S, Beneduce AA, Di Carlo V. Fast-track recovery programme after pancreatico- duodenectomy reduces delayed gastric emptying. Br J Surg 2008;95:1387-1393.

10. Aldameh A, McCall JL, Koea JB. Is routine placement of surgical drains necessary after elective hepatectomy? Results from a single institution. J Gastrointest Surg. 2005;9:667-671.

11. Fearon KC, Ljungqvist O, Von Meyenfeldt M, Revhaug A, Dejong CH, Lassen K, Nygren J, Hausel J, Soop $\mathrm{M}$, Andersen J, Kehlet $\mathrm{H}$. Enhanced recovery after surgery: a consensus review of clinical care for patients undergoing colonic resection. Clin Nutr. 2005;24:466-477.

12. Coolsen MM, Clermonts SH, van Dam RM, Winkens B, Malagó M, Fusai GK, Dejong CH, Olde Damink SW. Development of a composite endpoint for pancreaticoduodenectomie. accepted for publication in World J Surg. 2012 dec.

13. Lassen K, Coolsen MM, Slim K, Carli F, de Aguilar-Nascimento JE, Schäfer M, Parks RW, Fearon KC, Lobo DN, Demartines N, Braga M, Ljungqvist O, Dejong $\mathrm{CH}$; ERAS ${ }^{\circledR}$ Society; European Society for Clinical Nutrition and Metabolism; International Association for Surgical Metabolism and Nutrition. Guidelines for perioperative care for pancreaticoduodenectomy: Enhanced Recovery After Surgery (ERAS $\left({ }^{\circledR}\right)$ ) Society recommendations. Clin Nutr. 2012;31:817-830.

14. Bassi C, Dervenis C, Butturini G, Fingerhut A, Yeo C, Izbicki J, Neoptolemos J, Sarr M, Traverso W, Buchler M; International Study Group on Pancreatic Fistula Definition. Postoperative pancreatic fistula: An international study group (ISGPF) definition. Surgery 2005;138:8-13.

15. Welsch T, Eisele H, Zschäbitz S, Hinz U, Büchler MW, Wente MN. Critical appraisal of the International Study Group of Pancreatic Surgery (ISGPS) consensus definition of postoperative hemorrhage after pancreatoduodenectomy. Langenbecks Arch Surg 2011;396:783-791 


\section{PART III}

Evaluation of implementing an ERAS program in liver and pancreatic surgery 


\section{CHAPTER}

\section{Initial experience with a multimodal enhanced \\ recovery program in patients undergoing liver}

resection

RM van Dam, PO Hendry, MME Coolsen, MHA Bemelmans, K Lassen, A Revhaug, $\mathrm{KCH}$ Fearon, OJ Garden and CHC Dejong, on behalf of the Enhanced Recovery After Surgery (ERAS) Group

British Journal of Surgery 2008; 95: 969-975 


\section{ABSTRACT}

\section{Background}

Accelerated recovery from surgery has been achieved when patients are managed within a multimodal Enhanced Recovery After Surgery (ERAS) protocol. This study evaluated the benefit of an ERAS program for patients undergoing liver resection.

\section{Methods}

The ERAS protocol of epidural analgesia, early oral intake and early mobilization was studied prospectively in a consecutive series of 61 patients. Outcomes were compared with those in a consecutive series of 100 patients who underwent liver resection before the start of the study. Endpoints were postoperative length of hospital stay, postoperative resumption of oral intake, readmissions, morbidity and mortality.

\section{Results}

Fifty-six patients (92\%) in the ERAS group tolerated fluids within $4 \mathrm{~h}$ of surgery and a normal diet on day 1 after surgery. Median hospital stay, including readmissions, was 6.0 days compared with 8.0 days in the control group $(P<0.001)$. There were no significant differences in rates of readmission ( 13 and $10 \%$ respectively), morbidity ( 41 and $31 \%$ ) and mortality ( 0 and $2 \%$ ) between ERAS and control groups.

\section{Conclusions}

The ERAS fast-track protocol is safe and effective for patients undergoing liver resection. It allows early oral intake, promotes faster postoperative recovery and reduces hospital stay. 


\section{INTRODUCTION}

In the past two decades, the application of multimodal perioperative care protocols, commonly referred to as fast-track or enhanced recovery programs, has achieved significant improvements in the outcome of a variety of surgical procedures. By targeting factors that delay postoperative recovery (pain, gut dysfunction and immobility), and combining a series of interventions to reduce perioperative stress and organ dysfunction, such programs have been shown to accelerate postoperative recovery and reduce length of hospital stay. This can be achieved with an unaltered or even reduced rate of postoperative complications ${ }^{1}$. Protocols based on postoperative pain control through continuous mid- thoracic epidural anesthesia, stimulation of gut motility, early physical reactivation, and limited use of catheters, tubes and drains have resulted in improved convalescence for many surgical procedures, including colonic surgery, open aortic aneurysm repair and esophageal surgery ${ }^{2-7}$. In the past 5 years, the protocol in elective colonic surgery established by the Enhanced Recovery After Surgery (ERAS) Group has led to a reduction in median hospital stay from 9 to 5 days ${ }^{6}$.

Within a traditional perioperative care pathway median hospital stay following liver surgery ranges from 8 to 14 days $^{8-11}$. Morbidity is reasonably predictable, with hemorrhagic complications occurring predominantly during surgery or in the early postoperative phase, and biliary complications, intra-abdominal abscess or liver failure in the later postoperative phase $\mathrm{in}^{12,13}$. It was hypothesized that application of an enhanced recovery program for patients undergoing liver surgery would result in accelerated recovery and a shorter hospital stay. The main aim of the present study was to test the feasibility, safety and effectiveness of an ERAS protocol in patients undergoing liver surgery in two tertiary referral liver units in Europe.

\section{METHODS}

The ERAS liver study was initiated within the collaborative ERAS Group, and conducted in two units that perform high-volume liver surgery. The study was a prospective case series implementation study comparing outcomes with those in a historical control group.

The ERAS patient group comprised a consecutive series of 61 patients admitted to either the liver unit of Maastricht University Medical Center, The Netherlands, between 15 February 2005 and 31 August 2006, or the liver unit of the Royal Infirmary of Edinburgh, UK, where patient recruitment started on 1 February 2006. The control group consisted of a consecutive series of 100 patients who had liver resection in one of the two units between 1 July 2003 and 31 December 2004 (50 consecutive patients from each unit), when a more traditional care pathway was still in use. Patient and outcome data were derived from a prospectively collected database of all liver 
resections per-formed since 1 January 2000 in both units. In both groups, patients undergoing elective liver resection for primary or secondary tumors were considered eligible for the study if they had normal underlying liver function. Patients were excluded if they required biliary reconstruction or had emergency surgery.

In both units, the same group of liver surgeons per-formed the liver resections in the ERAS group as well as in the control group. In Maastricht more fellows with hepatopancreatobiliary training operated in the ERAS group. Bilateral subcostal transverse abdominal incisions were used. The transection plane was determined by intraoperative ultrasonography. To avoid excessive blood loss central venous pressure was reduced to below $5 \mathrm{mmHg}$ during transection using the Cavitron Ultrasonic Surgical Aspirator (CUSA ${ }^{\circledR}$; Valleylab, Boulder, Colorado, USA) and argon beam coagulation. The portal pedicles supplying and veins draining the sectors to be resected were divided and ligated with a running polypropylene suture. After removal of the liver specimen, the raw surface of the liver remnant was subjected to argon beam coagulation and sealed with TachoSil ${ }^{\circledR}$ (Nycomed, Zurich, Switzerland). In accordance with the protocol, no abdominal drains were placed in the ERAS group and the abdomen was closed with a standard running suture.

Before the introduction of the ERAS program neither of the institutions had a written, agreed perioperative care pathway. There were no specific measures to avoid prolonged preoperative and postoperative fasting, nasogastric decompression, excessive use of intravenous fluids and systemic opioids, prophylactic abdominal drains and postoperative immobilization. This conventional postoperative care program emphasized prolonged rest for both the patient and the gastrointestinal tract.

The ERAS multimodal evidence-based recovery program protocol, which was designed originally for elective colonic surgery, was modified to cover all aspects of elective liver resection ${ }^{2}$. Details of the ERAS liver protocol are given in Table 8.1.

In both groups, data were obtained prospectively during the hospital stay and for 90 days after surgery. Data recorded for each patient included morbidity, defined as complications related to the liver surgery, mortality, readmissions, postoperative hospital stay, use of preoperative chemotherapy, duration of surgical procedure, blood loss, anesthetic agents used and type of hepatectomy, defined according to the Brisbane 2000 terminology ${ }^{14}$. In the ERAS group, data on location of the epidural catheter, removal of the nasogastric tube if used, and time to resumption of oral intake and mobilization were also noted.

The primary endpoint of the study was total length of hospital stay, defined as the number of nights spent in hospital, including nights after readmission within 30 days after surgery. To facilitate objective assessment of clinical outcome and to prevent discharge too soon, predefined discharge criteria were used in the ERAS group (Table 8.2). Patients were informed about the protocol at a preadmission counseling session, where the importance of early mobilization and early oral intake were explained. Patients were discharged only if they met the discharge criteria and follow-up within 
3 days was possible in the outpatient clinic. Patients were given the mobile telephone number of the operating consultant surgeon to allow direct communication and safe deployment of the protocol.

Table 8.1 Day to day care plan of patients undergoing liver resection in the ERAS program

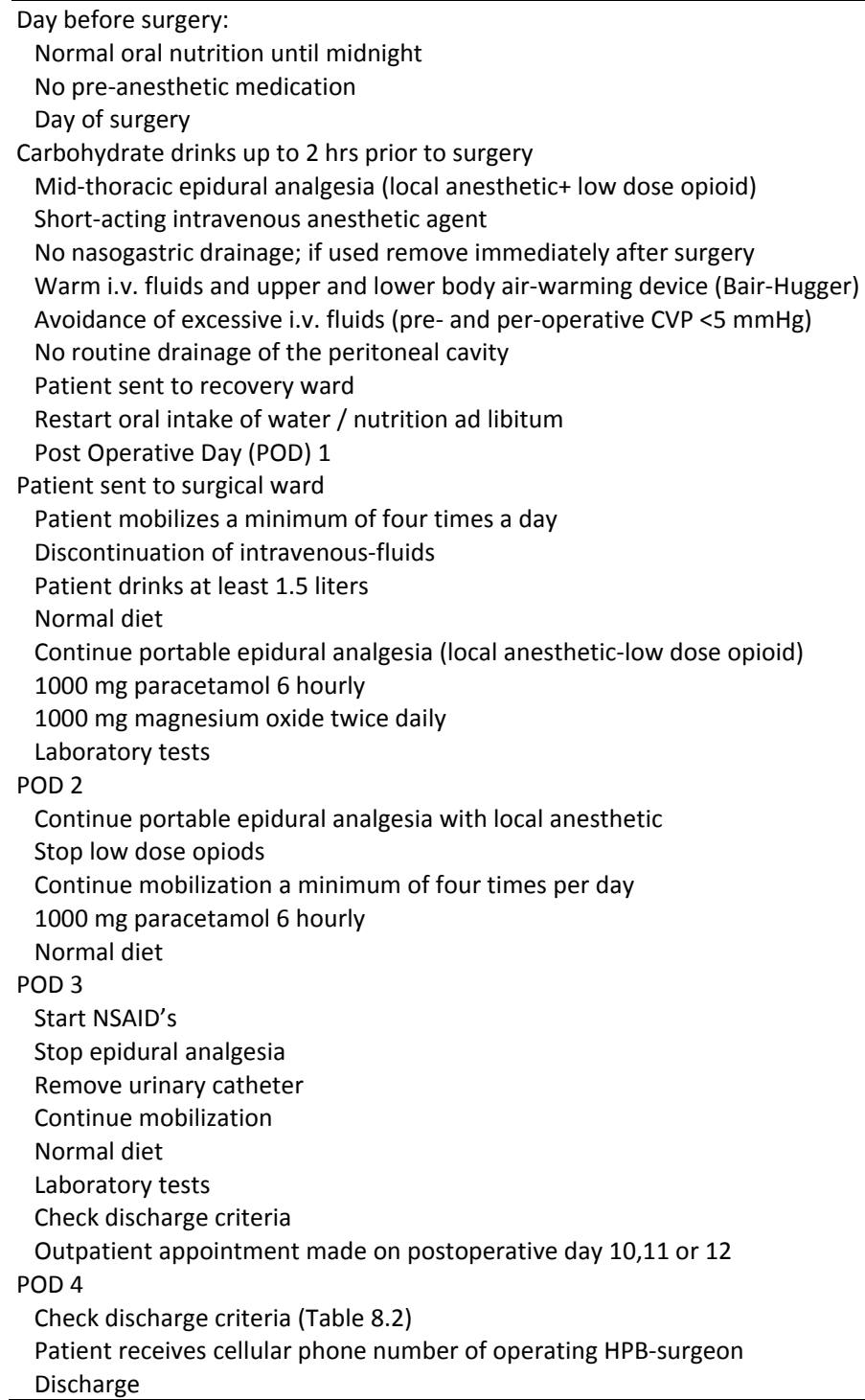


Table 8.2 Discharge criteria ERAS liver protocol

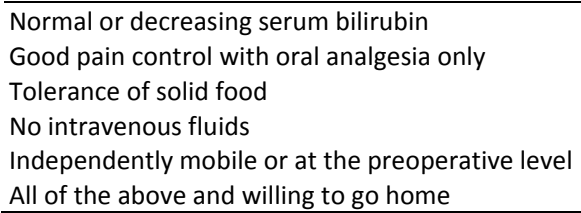

Patients in the control group were discharged without formal discharge criteria. The following secondary endpoints were evaluated: resumption of oral intake, defined as oral intake of water or normal food without discontinuation for at least $24 \mathrm{~h}$, readmissions, total morbidity, death and length of primary hospital stay, defined as the number of nights spent in hospital after surgery, excluding readmissions. Complications were registered during the primary admission or at subsequent outpatient clinic visits, after at least 6 and 30 days, using departmental liver registry databases.

\section{Statistical analysis}

Continuous data are expressed as median (range). Hospital stay, time to resumption of oral intake and postoperative duration of epidural analgesia were analyzed using the Mann-Whitney $U$ test. Readmission, complication and mortality rates were analyzed using $\div^{2}$ test or Fisher's exact test. $P<0.050$ was considered to be statistically significant. Data were analyzed using SPSS ${ }^{\circledR}$ version 13.0 for Windows ${ }^{\circledR}$ (SPSS, Chicago, Illinois, USA).

\section{RESULTS}

Patient characteristics were similar in the ERAS and control groups (Table 8.3). Types of liver resection are shown in Table 8.4. A higher proportion of patients in the ERAS group had repeat liver resections ( $11 \%$ versus $5.0 \%$ in control group; $P=0.214$ ) and resections after intensive chemotherapy (62 versus $33.0 \% ; P<0.001$ ).

The median (range) duration of surgery was 220 (60-420) min in the ERAS group and 270 (106-510) $\mathrm{min}$ in the control group $(P<0.001)$, and blood loss during surgery was $750(0-5000)$ and $800(0-6000) \mathrm{ml}$ respectively $(P=0.758)$. Abdominal drains were used significantly less often in the ERAS group ( 2 versus $66.0 \% ; P<0.001$ ).

In Edinburgh, the use of a nasogastric decompression tube in patients having partial liver resection had already been discontinued before 1 July 2003. In Maastricht, nasogastric decompression tubes were still used in most control patients. A nasogastric tube was inserted at induction in 37 of 47 patients in the ERAS group, significantly less frequently than in the control group $(96 \%)(P=0.013)$. In the ERAS group, the 
nasogastric tube was removed at the end of surgery or in the recovery ward within $4 \mathrm{~h}$ of surgery.

Oral intake was resumed within $4 \mathrm{~h}$ after surgery in 56 patients (92\%) in the ERAS group. Two patients required reinsertion of a nasogastric tube. The median (range) time to successful resumption of normal diet was $1(0-3)$ day in the ERAS group, compared with $3(0-14)$ days in controls $(P<0.001)$. Three patients in the ERAS group (5\%) became constipated after postoperative day 3 , despite initial tolerance of normal food, but this resolved within 5 days by restricting intake.

Table 8.3 Patient demographics ERAS liver study

\begin{tabular}{lcc}
\hline & ERAS & CONTROLS \\
& $\mathrm{N}=61$ & $\mathrm{~N}=100$ \\
\hline Sex (male: female) & $35: 26$ & $51: 49$ \\
Age median (range) & 62 years $(24-82)$ & 60 years $(20-81)$ \\
ASA 1 & $9(19 \%)$ & $14(14 \%)$ \\
ASA 2 & $31(66 \%)$ & $64(64 \%)$ \\
ASA 3 & $7(15 \%)$ & $22(22 \%)$ \\
Median blood loss (range) & $750 \mathrm{mls}(0-5000)$ & $800 \mathrm{mls}(0-6000)$ \\
Median duration of surgery (range) & $220 \mathrm{~min}(60-420)$ & $270 \mathrm{~min}(106-510)$ \\
Colorectal metastasis & $51(83.6 \%)$ & $72(72.0 \%)$ \\
Other metastasis & $2(3.3 \%)$ & $4(4.0 \%)$ \\
Hepatocellular / cholangiocarcinoma & $4(6.6 \%)$ & $9(9.0 \%)$ \\
Benign lesions & $4(6.6 \%)$ & $14(14 \%)$ \\
T4-invasive tumors in liver & $0(0 \%)$ & $1(1 \%)$ \\
\hline
\end{tabular}

ASA: American Society of Anesthesiologists, preoperative risk classification

Table 8.4 Type of liver resection

\begin{tabular}{lcc}
\hline & ERAS & Control \\
& $\mathrm{N}=61$ & $\mathrm{~N}=100$ \\
\hline Hemihepatectomy & $20(32.8 \%)$ & $38(38.0 \%)$ \\
Hemihepatectomy $+\geq 1$ metastasectomies & $6(9.8 \%)$ & $16(16.0 \%)$ \\
Extended hemihepatectomy & $7(11.5 \%)$ & $3(3 \%)$ \\
Multiple ( $\geq 2$ ) segmentectomy & $17(27.9 \%)$ & $18(18.0 \%)$ \\
Central resection / trisegmentectomy & $1(1.6 \%)$ & $4(4.0 \%)$ \\
Metastasectomy or mono-Segmentectomy & $10(16.4 \%)$ & $21(21.0 \%)$ \\
Repeat-hepatectomy $\%$ & $7(11.5 \%)$ & $5(5 \%)$ \\
\hline
\end{tabular}

Table 8.4b Specification of multiple $(\geq 2)$ segmentectomies

\begin{tabular}{lcc}
\hline & ERAS & Control \\
& $\mathrm{N}=61$ & $\mathrm{~N}=100$ \\
\hline Left lateral sectionectomy & $6(9.8 \%)$ & $9(9.0 \%)$ \\
Posterior sectionectomy & $1(1.6 \%)$ & 0 \\
Left lateral sectionectomy $+\geq 1$ metastasectomy & $2(3.3 \%)$ & 0 \\
Bisegmentectomy & $4(6.6 \%)$ & $5(5.0 \%)$ \\
$\geq 3$ segmentectomy & $4(6.6 \%)$ & $4(4.0 \%)$ \\
\hline
\end{tabular}


In the ERAS group, 58 patients (95\%) followed the anesthetic protocol of mid-thoracic epidural analgesia commencing before surgery ( $0.1 \%$ bupivacaine $+2 \mathrm{~g} / \mathrm{ml}$ fentanyl) with maintenance isoflurane or sevoflurane inhalation anesthesia, or intravenous propofol, as described previously ${ }^{15}$. After operation epidural analgesia comprising $0.1 \%$ bupivacaine and $2 \mu \mathrm{g} / \mathrm{ml}$ fentanyl was administered for at least 3 days via a portable patient-controlled device (GemStar ${ }^{\circledR}$ Infusion Systems; Hospira, Lake Forest, Illinois, UK) at a rate of $4-10 \mathrm{ml} / \mathrm{h}$, combined with oral non-opioid analgesics. This differed from anesthesiological management in the control group, where 89 patients $(89.0 \%)$ received epidural analgesia combined with isoflurane or sevoflurane inhalation anesthesia. In the postoperative period epidural analgesia consisting of $0.1 \%$ bupivacaine and $2 \mu \mathrm{g} / \mathrm{ml}$ fentanyl was administered without a specific time schedule at a daily fixed dose of $4-10 \mathrm{ml} / \mathrm{h}$, combined with intramuscular opioids (mainly piritramide) and oral analgesics. Postoperative epidural analgesia was continued for a median (range) of 3 (1-5) days in the ERAS group and 2 (0-6) days in the control group $(P<0.001)$. Epidural analgesia was discontinued earlier in the control group, and intramuscular or intravenous opioids (piritramide) were used more widely.

Outcome data are summarized in Table 8.5. There were no deaths in the ERAS group and one patient died within 30 days in the control group. A further control patient died after 62 days. Overall morbidity rates (percentage of patients with at least one complication) were 41 and $31.0 \%$ in the ERAS and control groups respectively $(P=0.197)$ (Table 8.6). All complications in the ERAS group were managed by non-surgical means. Readmission rates in ERAS and control groups were similar (13 versus 10.0\% respectively; $P=0.610$ ).

Table 8.5 Outcome of enhanced recovery program in liver surgery

\begin{tabular}{lccc}
\hline & ERAS & Control & $P$ \\
\hline MAASTRICHT ONLY* & $\mathrm{N}=47$ & $\mathrm{~N}=50$ & \\
Nasogastric tube (NGT)* & $37(78.7 \%)^{*}$ & $50(100 \%)^{*}$ & $P<0.013^{*}$ \\
Removal of NGT on day of surgery & $34 / 37(91.9 \%)^{*}$ & $0(0 \%)^{*}$ & $P<0.0001^{*}$ \\
MAASTRICHT \& EDINBURGH & & $\mathrm{N}=100$ & \\
Epidural analgesia & $\mathrm{N}=61$ & $89(89 \%)$ & $P=0.253$ \\
Abdominal drain & $58(95.1 \%)$ & $66(66 \%)$ & $P<0.0001$ \\
Complication rate & $1(1.6 \%)$ & $31(31.0 \%)$ & $P=0.233$ \\
Mortality & $25(41.0 \%)$ & $2(2.0 \%)$ & $P=0.526$ \\
Readmission rate & $0(0 \%)$ & $10(10.0 \%)$ & $P=0.610$ \\
Total length of stay (median, range), & $8(13.1 \%)$ & 8 days & $P<0.001$ \\
readmissions included) & 6 days & $(4-68)$ & $P<0.0001$ \\
Primary Hospital length of stay (median, range) & $(3-82)$ & 8 days & $(4-55)$ \\
\end{tabular}

* Maastricht only, in Edinburgh nasogastric tubes were not used in both patient groups. 
Table 8.6 Complications of enhanced recovery program in liver surgery

\begin{tabular}{lccc}
\hline & ERAS & Control & $P$-Value \\
& $\mathrm{N}=61$ & $\mathrm{~N}=100$ & \\
\hline Complications & $41.0 \%$ of patients & 31.0 of patients & $P=0.197$ \\
Bile leak & $4(6.6 \%)$ & $4(4.0 \%)$ & $P=0.479$ \\
Liver failure & $3(4.9 \%)$ & $1(1.0 \%)$ & $P=0.121$ \\
Sepsis & $0(0.0 \%)$ & $1(1.0 \%)$ & $P=0.433$ \\
Abdominal abscess & $5(8.2 \%)$ & $7(7.0 \%)$ & $P=0.779$ \\
Delayed GI function (<7 days) & $3(4.9 \%)$ & $1(2.5 \%)$ & $P=0.153$ \\
Pneumonia & $3(4.9 \%)$ & $2(2.0 \%)$ & $P=0.368$ \\
Pleural Effusion & $2(3.3 \%)$ & $2(2.0 \%)$ & $P=0.634$ \\
Pulmonary Embolism & $0(0 \%)$ & $1(1.0 \%)$ & $P=0.433$ \\
Myocardial Infarction & $2(3.3 \%)$ & $3(3.0 \%)$ & $P=0.921$ \\
Wound infection & $9(14.8 \%)$ & $5(5.0 \%)$ & $P<0.044$ \\
Other minor & $3(4.9 \%)$ & $16(20.0 \%)$ & $P<0.018$ \\
\hline
\end{tabular}

Fifty-two (85\%) of 61 patients in the ERAS group were completely mobile on the third day after operation and 29 (48\%) were discharged from hospital within 5 days. Median (range) total postoperative hospital stay, including readmissions, was 6.0 (3-82) days in the ERAS group and 8.0 (4-68) days in the control group $(P<0.001)$ (Figure 8.1).

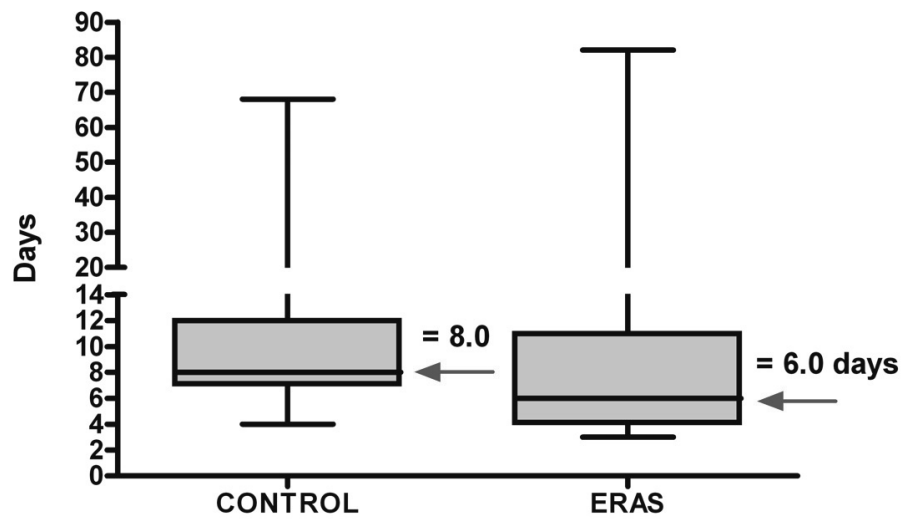

Figure 8.1 Total hospital length of stay (tLOS), readmissions included. Arrows = median tLOS in days, $P<0.001$, ERAS vs. control.

\section{DISCUSSION}

The present study showed that use of an evidence- based multimodal enhanced recovery program after liver resection accelerated postoperative recovery and led to a significantly shorter hospital stay. Patients managed according to the ERAS protocol were able to drink fluids within $4 \mathrm{~h}$ of liver resection and eat normal food on the day 
after surgery. Most patients were completely mobile after 3 days and, as a consequence, almost half of the patients were discharged from hospital within 5 days. Median total hospital stay (including readmissions) was reduced from 8 to 6 days, which is considerably shorter than the 8-14 days reported by other European centers $^{8-10}$. Application of the ERAS protocol did not have any detrimental effect on safety in liver resection.

Based on previous experience of enhanced recovery implementation studies within these units, and attempts to randomize between fast-track and traditional care in a multicenter abdominal surgery trial ${ }^{6,16}$, a randomized clinical trial comparing fast-track and traditional care was considered impractical. Both units had implemented ERAS protocols over the previous few years and, in view of the growing evidence base for such programs, a traditional perioperative protocol was felt to represent suboptimal care. More importantly, it would have been necessary for nursing and surgical staff to return to traditional perioperative care pathways. Therefore, this feasibility study was designed as a prospective case series with a historical control group.

Several important issues have become apparent from this study. Importantly, adverse outcomes, assessed in terms of readmissions, complications and deaths, were similar in the ERAS and control groups. The complication rate in the ERAS group is in keeping with recently published rates of $23-52 \%{ }^{10,17-20}$. Although the morbidity rate appeared to be higher in the ERAS group, the study was not sufficiently powered to determine statistical differences in the incidence of complications. Moreover, complications directly related to liver surgery, such as bleeding, bile leakage or temporary liver failure, are unlikely to be influenced by postoperative care. Avoidance of abdominal drains in this study might arguably have influenced the complication rate, but the authors consider this unlikely because the incidence of bile leak and intra-abdominal abscess was similar in the two groups. Prophylactic drains are employed in many centers to detect early complications such as postoperative hemorrhage or bile leakage, to remove intra-peritoneal fluids and to prevent abscess formation. However, there is evidence that abdominal drainage after liver resection does not reduce the incidence of re-interventions for these complications ${ }^{21-23}$. Some studies have even noted higher rates of infected collections when drainage was used ${ }^{13,21,24}$. This suggests that drains might be detrimental to clinical outcome by providing a route for ascending infections. Another disadvantage of drains within an enhanced recovery setting is that they represent a significant impediment to achieving early mobilization.

A few elements have proven difficult to implement universally. The use of carbohydrate drinks before surgery reduces thirst, hunger and anxiety, and is safe up to $2 \mathrm{~h}$ before abdominal surgery ${ }^{25}$. However, a preoperative fasting policy of 'nil by mouth from midnight' is still common. Owing to tradition in the anesthesia department nasogastric decompression tubes were still used extensively during surgery in both groups in Maastricht, but were removed immediately after surgery in the ERAS group. This practice meant a major change in clinical routines, and did not appear to result in more 
pulmonary complications in the present study. In general, nasogastric decompression is still used widely following abdominal surgery ${ }^{26}$, despite evidence that it is unnecessary and can result in more pulmonary complications. Further support for avoidance of nasogastric tubes specifically in liver surgery has been published recently ${ }^{11}$.

Early postoperative enteral nutrition compared with 'nil by mouth' improves clinical outcome $^{27}$. In the present study, oral intake of water within $4 \mathrm{~h}$ after surgery and institution of normal nutrition on the day after operation were well tolerated in the ERAS group. Early resumption of normal diet in combination with other elements of the enhanced recovery program is designed to reduce the occurrence of delayed gastrointestinal function after surgery and even promote appetite. Therefore, the ability of the patient to tolerate normal food is an outcome in itself. A period of delayed gastrointestinal functioning after operation, observed in a few patients, was registered as a complication. It might also be argued that a delay in gastrointestinal functioning is a physiological phenomenon that occurs after abdominal surgery in many patients. A restrictive perioperative intravenous fluid regimen may help to reduce the occurrence of delayed gastrointestinal functioning ${ }^{28}$. Fluid restriction may well be an important issue in hepatic surgery and is the subject of ongoing research in the authors' units.

It is important that surgical patients are treated in an environment that encourages early mobilization ${ }^{4}$. Mobilization on the day of surgery was possible in Edinburgh, but was not achieved in Maastricht because the recovery unit did not contain the appropriate facilities. Adequate pain control and a substantial effort by nursing staff are required to achieve early mobilization.

In conclusion, a multimodal enhanced recovery program for patients undergoing liver resection for primary and secondary tumors, but with otherwise normal hepatic function, is feasible and effective. Patients were able to drink within $4 \mathrm{~h}$ of surgery, eat on the following day and were usually mobile by the third day after operation. This is indicative of earlier recovery and was accompanied by a $25 \%$ reduction in postoperative hospital stay. The fact that only half of the patients were discharged within 5 days indicates that there is further room for improvement. 


\section{REFERENCES}

1. Kehlet H, Dahl JB. Anaesthesia, surgery, and challenges in postoperative recovery. Lancet 2003;362: 1921-1928.

2. Fearon KC, Ljungqvist O, Von Meyenfeldt M, Revhaug A, Dejong CH, Lassen K, Nygren J, Hausel J, Soop $\mathrm{M}$, Andersen J, Kehlet $\mathrm{H}$. Enhanced recovery after surgery: a consensus review of clinical care for patients undergoing colonic resection. Clin Nutr 2005;24: 466-477.

3. Podore PC, Throop EB. Infrarenal aortic surgery with a 3-day hospital stay: a report on success with a clinical pathway. J Vasc Surg 1999;29: 787-792.

4. Kehlet H, Wilmore DW. Multimodal strategies to improve surgical outcome. Am J Surg 2002;183: 630-641.

5. Nygren J, Hausel J, Kehlet H, Revhaug A, Lassen K, Dejong C, Andersen J, von Meyenfeldt M, Ljungqvist $\mathrm{O}$, Fearon KC. A comparison in five European Centres of case mix, clinical management and outcomes following either conventional or fast-track perioperative care in colorectal surgery. Clin Nutr 2005;24:455-461.

6. Maessen J, Dejong CH, Hausel J, Nygren J, Lassen K, Andersen J, Kessels AG, Revhaug A, Kehlet H, Ljungqvist $\mathrm{O}$, Fearon $\mathrm{KC}$, von Meyenfeldt MF. A protocol is not enough to implement an enhanced recovery programme for colorectal resection. Br J Surg 2007;94: 224-231.

7. Cerfolio RJ, Bryant AS, Bass CS, Alexander JR, Bartolucci AA. Fast tracking after Ivor Lewis esophagogastrectomy. Chest 2004;126: 1187-1194.

8. Capussotti L, Muratore A, Ferrero A, Massucco P, Ribero D, Polastri R. Randomized clinical trial of liver resection with and without hepatic pedicle clamping. Br J Surg 2006;93:685-689.

9. Dimick JB, Wainess RM, Cowan JA, Upchurch GR JR, Knol JA, Colletti LM. National trends in the use and outcomes of hepatic resection. J Am Coll Surg 2004;199:31-38.

10. Jarnagin WR, Gonen M, Fong Y, DeMatteo RP, Ben-Porat L, Little S, Corvera C, Weber S, Blumgart LH. Improvement in perioperative outcome after hepatic resection: analysis of 1803 consecutive cases over the past decade. Ann Surg 2002;236:397-406.

11. Pessaux P, Regimbeau JM, Dondero F, Plasse M, Mantz J, Belghiti J. Randomized clinical trial evaluating the need for routine nasogastric decompression after elective hepatic resection. Br J Surg 2007;94: 297-303.

12. Belghiti J, Hiramatsu K, Benoist S, Massault P, Sauvanet A, Farges O. Seven hundred forty-seven hepatectomies in the 1990s: an update to evaluate the actual risk of liver resection. J Am Coll Surg 2000;191:38-46.

13. Burt BM, Brown K, Jarnagin W, DeMatteo R, Blumgart LH, Fong Y. An audit of results of a no-drainage practice policy after hepatectomy. Am J Surg 2002;184:441-445.

14. Strasberg SM. Nomenclature of hepatic anatomy and resections: a review of the Brisbane 2000 system. J Hepatobiliary Pancreat Surg 2005;12:351-355.

15. van de Poll MC, Siroen MP, van Leeuwen PA, Soeters PB, Melis GC, Boelens PG, Deutz NE, Dejong CH. Interorgan amino acid exchange in humans: consequences for arginine and citrulline metabolism. $A m \mathrm{~J}$ Clin Nutr 2007;85:167-172.

16. Wind J, Hofland J, Preckel B, Hollmann MW, Bossuyt PM, Gouma DJ, van Berge Henegouwen MI, Fuhring JW, Dejong CH, van Dam RM, Cuesta MA, Noordhuis A, de Jong D, van Zalingen E, Engel AF, Goei TH, de Stoppelaar IE, van Tets WF, van Wagensveld BA, Swart A, van den Elsen MJ, Gerhards MF, de Wit LT, Siepel MA, van Geloven AA, Juttmann JW, Clevers W, Bemelman WA. Perioperative strategy in colonic surgery; LAparoscopy and/or FAst track multimodal management versus standard care (LAFA trial). BMC Surg 2006;6:16.

17. Petrowsky H, McCormack L, Trujillo M, Selzner M, Jochum W, Clavien PA. A prospective, randomized, controlled trial comparing intermittent portal triad clamping versus ischemic preconditioning with continuous clamping for major liver resection. Ann Surg 2006;244:921-928.

18. Figueras J, Llado L, Miro M, Ramos E, Torras J, Fabregat J, Serrano T. Application of fibrin glue sealant after hepatectomy does not seem justified: results of a randomized study in 300 patients. Ann Surg 2007;245: 536-542. 
19. Schroeder RA, Marroquin CE, Bute BP, Khuri S, Henderson WG, Kuo PC. Predictive indices of morbidity and mortality after liver resection. Ann Surg 2006;243:373-379.

20. Benzoni E, Cojutti A, Lorenzin D, Adani GL, Baccarani U, Favero A, Zompicchiati A, Bresadola F, Uzzau A. Liver resective surgery: a multivariate analysis of postoperative outcome and complication. Langenbecks Arch Surg 2007;392: 45-54.

21. Fong Y, Brennan MF, Brown K, Heffernan N, Blumgart LH. (1996) Drainage is unnecessary after elective liver resection. Am J Surg 1996;171:158-162.

22. Petrowsky H, Demartines N, Rousson V, Clavien PA. Evidence-based value of prophylactic drainage in gastrointestinal surgery: a systematic review and meta-analyses. Ann Surg 2004;240: 1074-1084.

23. Belghiti J, Kabbej M, Sauvanet A, Vilgrain V, Panis Y, Fekete F. Drainage after elective hepatic resection. A randomized trial. Ann Surg 1993;218:748-753.

24. Liu CL, Fan ST, Lo CM, Wong Y, Ng IO, Lam CM, Poon RT, Wong J. Abdominal drainage after hepatic resection is contraindicated in patients with chronic liver diseases. Ann Surg 2004;239:194-201.

25. Brady M, Kinn S, Stuart P. Preoperative fasting for adults to prevent perioperative complications. Cochrane Database Syst Rev 2003;4:CD004423.

26. Kehlet H, Buchler MW, Beart RW JR, Billingham RP, Williamson R. Care after colonic operation - is it evidence-based? Results from a multinational survey in Europe and the United States. J Am Coll Surg 2006;202:45-54.

27. Lewis SJ, Egger M, Sylvester PA, Thomas S. Early enteral feeding versus 'nil by mouth' after gastrointestinal surgery: systematic review and meta-analysis of controlled trials. BMJ 2001;323: 773-776.

28. Lobo DN, Bostock KA, Neal KR, Perkins AC, Rowlands BJ, Allison SP. Effect of salt and water balance on recovery of gastrointestinal function after elective colonic resection: a randomised controlled trial. Lancet 2002;359:1812-1818. 


\section{CHAPTER}

Improving outcome after pancreaticoduodenectomy experiences with implementing an enhanced recovery after surgery program

MME Coolsen, RM van Dam, A Chigharoe, SWM Olde Damink, CHC Dejong Digestive Surgery 2014;31:177-184 


\begin{abstract}
Background

Pancreaticoduodenectomies (PDs) are complex surgical procedures that require high standard perioperative care. The objective of this study was to evaluate the effects on patient outcome of implementing an Enhanced Recovery After Surgery (ERAS) program for PD on patient outcome.
\end{abstract}

\title{
Methods
}

230 patients undergoing PD in Maastricht University Medical Center between January 1995 and January 2012 were included. Group 1 (1995-2005) received traditional care. From January 2006 several elements of an ERAS pathway for pancreatic surgery were implemented (group 2: 'ERASlike'). From 2009 onwards the ERAS pathway was fully implemented (group 3: ERAS). Mortality, complications, readmissions and length of hospital stay (LOS) were evaluated in the subgroups and compared.

\section{Results}

Median LOS was significantly reduced from 20 days in group 1, to 13 days in group 2 and 14 days in group $3(P=0.001)$. Median LOS of patients without complications was 16,10 and 9 days, respectively, in group 1,2 and $3(P<0.0001)$. Over time, the average age of patients undergoing PD increased significantly. Complication rates as well as mortality and readmission rates did not change over time.

\section{Conclusion}

Implementing an ERAS program contributed to a decrease of LOS without compromising other outcomes. Mortality, morbidity and readmission rates stayed unchanged and more complications were managed non-operatively. 


\section{INTRODUCTION}

Pancreaticoduodenectomy (PD) is a technically challenging surgical procedure and is currently the only curative treatment for malignancy in the periampullary region. In Whipple's original report on 41 pancreaticoduodenectomies, a mortality rate of $29 \%$ was recorded ${ }^{1}$. Nowadays, centralization of PD patients in specialized centers and advances in perioperative care have resulted in mortality rates of less than $5 \%$. Morbidity rates, however, have remained as high as $40-60 \%$.

In order to further improve short-term outcome, enhanced recovery after surgery (ERAS) programs (also called "Fast Track") have been developed. These multimodal perioperative programs involving close collaboration between nurses, surgeons, anesthetists and other paramedic caregivers have received increasing interest over the last decades. The ERAS concept was initiated by Kehlet ${ }^{5}$ in the early 1990s for colonic surgery. Several other fields of surgery have since adopted the ERAS program achieving better postoperative outcome and decreasing length of postoperative hospitalization ${ }^{6-}$ ${ }^{12}$. In pancreatic surgery, a recent systematic review and meta-analysis suggested that an ERAS program reduces hospital stay and morbidity for $\mathrm{PD}^{13}$. Also, in another study of 145 patients undergoing pancreatic surgery implementing an ERAS program appeared to be feasible and safe ${ }^{14}$. An independent factor associated with early discharge in this study was early resumption of a normal diet and the lack of jaundice. In the Maastricht University Medical Center (MUMC), the ERAS program for colonic surgery was implemented from 2000 onwards $^{15}$. In 2005, a pilot began in Maastricht resulting in the successful implementation of the ERAS program in liver surgery ${ }^{16}$. The impact of the ERAS liver program became more evident at the end of the 2006 pilot. Several ERAS elements were also implemented in pancreatic surgery but a standardized protocol was not available at that time. In 2009 a concept protocol was established and has been used ever since. The ERAS pancreas collaborative group, in which MUMC also participates, recently developed a consensus protocol for ERAS in pancreaticoduodenectomy ${ }^{17}$. This consensus protocol will be implemented from 2013 onwards and will be evaluated in a multicenter international prospective trial. The objective of the present study was to examine patient characteristics, indications and outcome after PD in the period from 1995 to 2011 and also to evaluate the effects of implementing the concept ERAS protocol for pancreaticoduodenectomy.

\section{PATIENTS AND METHODS}

All patients undergoing pancreaticoduodenectomy in MUMC between January 1995 and January 2012 were reviewed retrospectively by two authors (MC and AC). Four experienced surgeons carried out the surgical procedures. The procedure most commonly performed was pylorus-preserving PD with end-to-side pancreatico- 
jejunostomy, end-to-side hepaticojejunostomy and retrocolic duodenojejunostomy. In case of distal stomach involvement or proximal duodenum a classical Whipple's procedure was performed. All anastomoses were hand-sewn. Until 2006 two drains were placed at the end of the procedure in the foramen of Winslow and near the pancreaticojejunostomy. From 2006, the left-sided drain was omitted and only one drain was placed near the pancreaticojejunostomy. Jejunostomy catheters were used until 2007. Octreotide was not given routinely.

From January 2006, a subset of ERAS protocol elements was implemented for all patients undergoing PD (epidural analgesia, early mobilization, use of laxatives, earlier removal of NG tube and resumption of intake). However, patients were not managed in an official care pathway. From January 2009, a protocol for ERAS pancreatic surgery was more rigorously implemented for all patients undergoing PD. The total population was therefore divided into three groups defined by these periods: Group 1 (1995-2005) "no ERAS", group 2 (2006-2008) "ERAS-like" and group 3 (2009-2011) "ERAS". Table 9.1 shows the elements of the ERAS program for pancreatico-duodenectomy applied to all patients in group 3 (ERAS). Patients managed in this ERAS program were discharged when medically fit for discharge, when functional recovery criteria were met, and if they were willing to go home. The functional recovery criteria included were good pain control with oral analgesia only, tolerance for solid food, no intravenous fluids, passage of stool, and being independently mobile at the pre-operative level.

All patients were entered into a database retrospectively from 1995 and from 2006 prospectively. The following data were extracted from the database: patient age, sex, co-morbidity, indication and type of surgery (classical Whipple's procedure or Pylorus Preserving Pancreaticoduodenectomy (PPPD)).

Postoperative outcomes were length of hospital stay (LOS), mortality, readmissions and complications. Definitions used for specific complications were according to literature standards (Appendix 5) ${ }^{18-23}$. Data on postoperative interventions (radiological or surgical) were also extracted from the database. From 1995 until 2000 complications were registered once a week on paper. Since 2000 a national digital complication registration system for surgery was implemented and complications were registered prospectively. Complications were registered on paper on a daily basis and then entered into in the national digital complication system by a secretary. As of 2009 this national registration system was linked to the electronic patient dossier and complications were completely registered digitally. Complications were classified using the Dindo-Clavien severity grading system ${ }^{24}$. Mortality, readmissions and all complications were crosschecked with our complication registration office and adjusted when necessary. 
Table 9.1 Protocol for the enhanced recovery after surgery (ERAS) program for PD

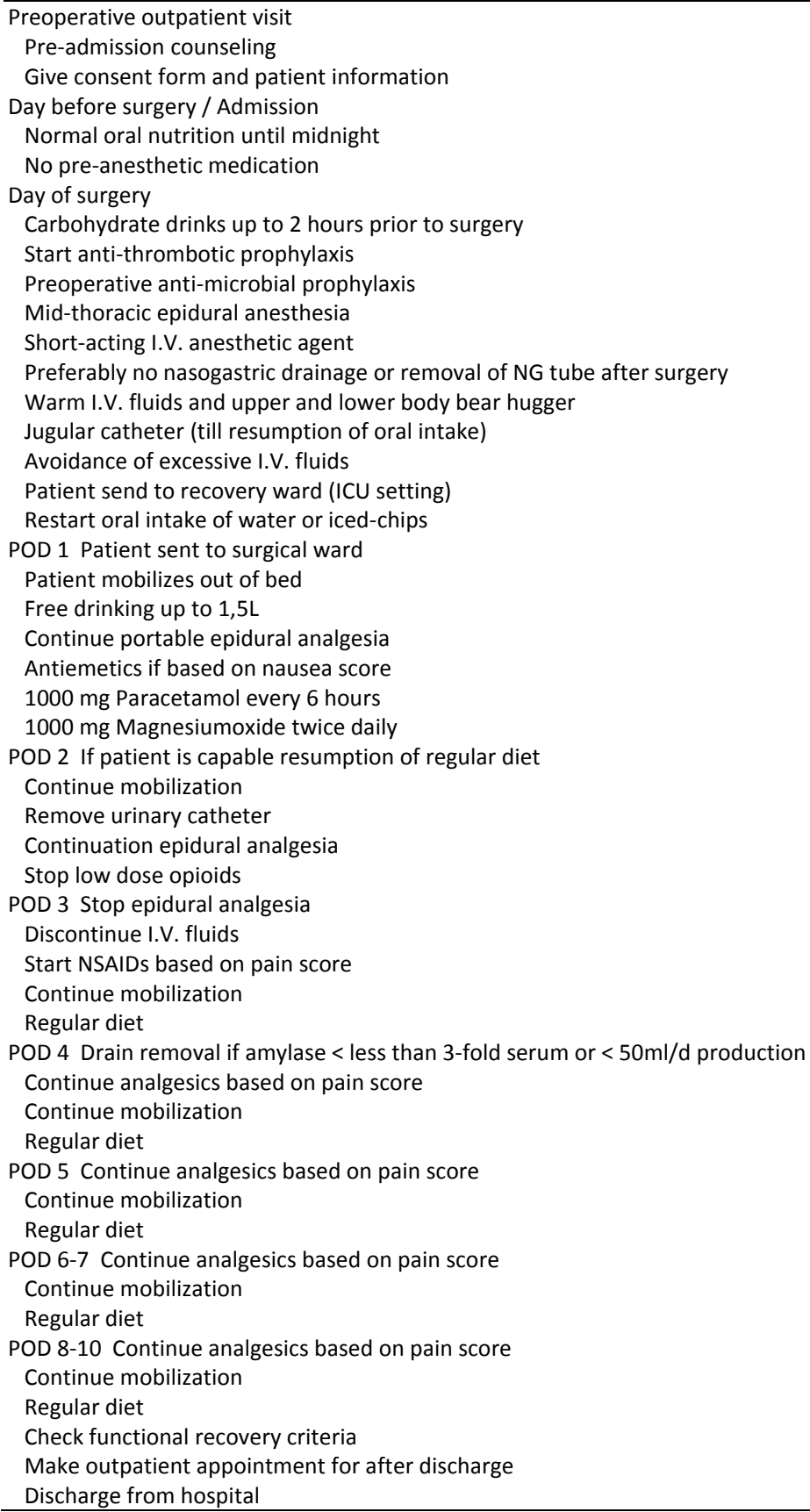




\section{Statistical analysis}

Statistical analysis was performed using SPSS for Windows, version 19. Patient age was analyzed within two groups using an unpaired t-test while the overall significance within all three groups was analyzed using a one-way ANOVA test. All other baseline characteristics and results except LOS were analyzed using a Chi-square or Fisher's exact test. LOS was analyzed between two groups using the Mann-Whitney $U$ test, whereas overall significance between all three groups was analyzed using the KruskalWallis test. LOS in various time periods was compared with multivariable linear regression. Logarithmic transformation of length of stay was necessary as this variable was not normally distributed. Baseline characteristics were entered into the multivariable regression model. The clinically relevant interaction term time period $x$ age was added to the regression model and removed if not statistically significantly associated with length of stay. Multicolinearity and influential outliers were checked using variance inflation factors and Cook's distance. A $P$-value $<0.05$ was considered significant.

\section{RESULTS}

\section{Patient and operative characteristics}

Demographic patient data and operative characteristics are shown in Table 9.2. Mean patient age was significantly higher in the most recently operated group (from $62 \pm 13$ to $61 \pm 11$ to $67 \pm 11, P=0.003$ ). There were no differences in male-female distribution between the three groups. Co-morbidity did not differ significantly between groups. Fewer patients were operated on for distal cholangiocarcinoma in the last group $(P=0.04)$. The ratio between classical Whipple procedure and PPPD was around 1:3 in the first two groups, with a ratio of approximately 1:4 in the last group (not significant). The PD resection volume in MUMC from 1995-2011 increased from an average of 8 PDs/year in the period of 1995-2000 to 29 PDs/year in the period 2009- 2011.

\section{Clinical outcomes and complications}

In groups 2 and 3, all patients received epidural analgesia and NG tubes were removed at the end of the procedure. Patients in groups 2 and 3 were structurally encouraged to mobilize with the help of a physiotherapist from postoperative day (POD) 1 . In group 3 patients structurally received sips of water in the recovery ward on the day of surgery and were encouraged to resume and gradually increase their normal diet from POD 1. Clinical postoperative outcomes and complications are shown in Table 9.3. LOS decreased from 20 days in group 1 to 13 days in group 2 and 14 days in group 3 $(P=0.001)$. LOS including readmissions was 22,14 and 16 days in the 3 groups 
respectively $(p=0.003)$. Table 9.4 shows that patients without complications had a LOS of 16,10 and 9 days respectively $(P<0.001)$. Reasons for delayed discharge despite fulfilling functional recovery criteria were mainly of logistic nature (e.g. no discharge in weekends and home care not yet being arranged). Separate analysis of LOS for patients with complications demonstrated that patients with a Dindo-Clavien grade $<3$ complication had a significantly longer LOS in group 1 compared to group 2 and 3 $(P=0.01)$. LOS of patients with a Dindo-Clavien grade $\geq 3$ did not differ significantly between groups. Mortality did not differ significantly between the groups, nor did the readmission rate. The incidence of complications such as pancreatic fistula, delayed gastric emptying (DGE), intra-abdominal abscesses, post pancreaticoduodenectomy hemorrhage, gastrojejunostomy and hepaticojejunostomy leakage and sepsis did not change significantly as time passed. The incidence of complications classified according to Dindo-Clavien also did not show any differences between the groups. Wound infections were significantly more common in the patients of group $3(P=0.008)$ Incidences of specific complications were calculated for PPPD and the classical Whipple procedure separately. There were no significant differences in occurrence of any of the complications between these two procedures (Appendix 6).

Table 9.2 Patient and operative characteristics

\begin{tabular}{|c|c|c|c|}
\hline & $\begin{array}{c}\text { No ERAS } \\
\text { 1995-2005 } \\
\text { Population } 1(n=97)\end{array}$ & $\begin{array}{c}\text { 'ERAS-like' } \\
\text { 2006-2008 } \\
\text { Population } 2(n=47)\end{array}$ & $\begin{array}{c}\text { ERAS } \\
\text { 2009-2011 } \\
\text { Population } 3(n=86) \\
\end{array}$ \\
\hline Age (mean)* & $62 \pm 13$ & $61 \pm 11$ & $67 \pm 11 \ddagger$ \\
\hline \multicolumn{4}{|l|}{ Sex } \\
\hline Male & $58(59.8)$ & $30(63.8)$ & $44(51.2)$ \\
\hline Female & $39(40.2)$ & $17(36.2)$ & $42(48.8)$ \\
\hline \multicolumn{4}{|l|}{ Co-morbidity } \\
\hline DM & $19(19.6)$ & 5 (10.6) & $17(19.8)$ \\
\hline Cardiovascular & $32(33.0)$ & $18(38.3)$ & $27(31.4)$ \\
\hline \multicolumn{4}{|l|}{ Indication } \\
\hline Pancreatic carcinoma & $38(39.2)$ & $22(46.8)$ & $45(52.3)$ \\
\hline Ampullary carcinoma & $16(16.5)$ & $6(12.8)$ & $19(22.1)$ \\
\hline Duodenum carcinoma & $5(5.2)$ & $3(6.4)$ & $4(4.7)$ \\
\hline Distal cholangiocarcinoma & $12(12.4)$ & $2(4.3)$ & $3(3.5) \ddagger$ \\
\hline Pancreatic pseudo cyst & $1(1,0)$ & $0(0)$ & $0(0)$ \\
\hline Peri-ampullary adenoma & $3(3.1)$ & $3(6.4)$ & $1(1.2)$ \\
\hline Neuro-endocrine tumor & $0(0)$ & $0(0)$ & $1(1.2)$ \\
\hline Benign pancreatic tumor & $5(5.2)$ & $5(10.6)$ & $4(4.7)$ \\
\hline \multicolumn{4}{|l|}{ Pancreatitis } \\
\hline Chronic & $4(4.1)$ & $2(4.2)$ & $1(1.2)$ \\
\hline Other & $13(13.4)$ & $4(8.5)$ & $8(9.3)$ \\
\hline \multicolumn{4}{|l|}{ Surgical technique } \\
\hline Whipple & $24(24.7)$ & $11(23.4)$ & $18(20.9)$ \\
\hline PPPD & $73(75.3)$ & $36(76.6)$ & $68(79.1)$ \\
\hline
\end{tabular}

Values in parentheses are in percentages unless indicated otherwise. ${ }^{*} \pm \mathrm{SD}$. $+P<0.05$ vs. group $1 . \ddagger P<0.05$ vs. group 1,2 
Table 9.3 Postoperative outcomes and complication

\begin{tabular}{|c|c|c|c|}
\hline & $\begin{array}{c}\text { No ERAS } \\
\text { 1995-2005 } \\
\text { Population } 1 \\
(n=97)\end{array}$ & $\begin{array}{c}\text { 'ERAS-like' } \\
\text { 2006-2008 } \\
\text { Population } 2 \\
(n=47)\end{array}$ & $\begin{array}{c}\text { ERAS } \\
2009-2011 \\
\text { Population } 3 \\
(n=86)\end{array}$ \\
\hline \multicolumn{4}{|l|}{ Complications } \\
\hline \multicolumn{3}{|l|}{ (Dindo-Clavien grade I-II) } & $20(23.3)$ \\
\hline \multicolumn{4}{|l|}{ (Dindo-Clavien grade IIla/b) } \\
\hline \multicolumn{4}{|l|}{ (Dindo-Clavien grade IVa/b) } \\
\hline Intra abdominal abscess & $15(15.5)$ & $6(12.8)$ & $14(16.3)$ \\
\hline Pancreatic fistula & $12(12.4)$ & $6(12.8)$ & $11(12.3)$ \\
\hline Grade A & $1(1.0)$ & $1(2.1)$ & $1(1.2)$ \\
\hline Grade B & $2(2.1)$ & $1(2.1)$ & $2(2.3)$ \\
\hline Grade C & $9(9.3)$ & $4(8.5)$ & $8(9.3)$ \\
\hline$D G E$ & $7(7.2)$ & $4(8.5)$ & $11(12.8)$ \\
\hline Grade A & $1(1.0)$ & $1(2.1)$ & $1(1.2)$ \\
\hline Grade B & $0(0)$ & $0(0)$ & $1(1.2)$ \\
\hline Grade C & $6(6.2)$ & $4(8.5)$ & $8(9.3)$ \\
\hline Biliodigestive anastomosis leakage & $5(5.2)$ & $4(8.5)$ & $4(4.7)$ \\
\hline Post-pancreatectomy hemorrhage & $3(3.1)$ & $1(2.1)$ & $7(8.1)$ \\
\hline Gastrojejunostomy leakage & $2(2.1)$ & $0(0)$ & $2(2.3)$ \\
\hline Sepsis/MOF & $4(4.1)$ & $2(4.3)$ & $5(5.8)$ \\
\hline Wound infection & $5(5.2)$ & $10(21.3)^{\dagger}$ & 15 (17.4)‡ \\
\hline General complications & 19 (19.6) & $12(25.5)$ & $29(33.7)$ \\
\hline \multicolumn{4}{|l|}{ Interventions } \\
\hline Percutaneous drainage & $13(13.4)$ & $8(17.0)$ & $16(18.6)$ \\
\hline Relaparotomy & $13(13.4)$ & $5(10.6)$ & $7(8.1)$ \\
\hline LOS (median) * & $20(9-132)$ & $13(7-62)^{\dagger}$ & $14(7-83)+$ \\
\hline LOS incl readmission (median) * & $22(9-132)$ & $14(7-62)+$ & $16(7-83)+$ \\
\hline Mortality & $6(6.2)$ & $1(2.1)$ & $4(4.7)$ \\
\hline Readmissions & $14(14.4)$ & $7(14.8)$ & $11(12.8)$ \\
\hline
\end{tabular}

Values in parentheses are in percentages unless indicated otherwise. ${ }^{*}$ range. $+P<0.05$ vs. group $1 . \neq P<0.05$ vs. group 1,21

Table 9.4 LOS of patients with and without complications, graded with the Dindo-Clavien classification

\begin{tabular}{lccc}
\hline LOS, median (range) & $\begin{array}{c}1995-2005 \\
\text { No ERAS }(n=97)\end{array}$ & $\begin{array}{c}2006-2008 \\
\text { 'ERAS like' }(n=47)\end{array}$ & $\begin{array}{c}2009-2011 \\
\text { ERAS }(n=86)\end{array}$ \\
\hline No complication & $16[9-44]$ & $10[7-18]^{\dagger}$ & $9[7-16] \ddagger$ \\
Complication grade Dindo-Clavien $<3$ & $26[10-70]$ & $12[8-23] \dagger$ & $16[8-49] \ddagger$ \\
Complication grade Dindo-Clavien $\geq 3$ & $32[12-133]$ & $37[11-62]$ & $36[12-83]$ \\
\hline
\end{tabular}

$\dagger P<0.05$ vs group $1 . \ddagger P<0.05$ vs group 1,2 


\section{ERAS outcomes for group 3: "ERAS group" versus group 1: "no ERAS"}

Patients in group $1(n=97)$ all received a NG tube and it was removed several days to weeks after surgery when no gastric retention was present. In the ERAS group NG tubes were not routinely placed. If a nasogastric tube was placed during surgery, it was removed directly after surgery in all patients. Patients were allowed to take sips of water on the day of surgery in the recovery ward. In 11 patients in the ERAS group $(12.8 \%)$ a NG tube had to be reinserted in the postoperative period to relieve symptoms of DGE.

All patients in the ERAS group $(n=86,100 \%)$ received epidural analgesia and it was stopped on POD 3 in $78 \%$ of the patients $(n=67)$. In the 'no ERAS' group intravenous analgesia was mainly used.

All patients received an intra-abdominal drain located at the pancreatico-jejunostomy at the end of the procedure. Intra-abdominal drain removal was at the discretion of the surgeon in the 'no ERAS' group. In the ERAS group drain removal criteria were used (Table 9.1). Drains were removed on day 4 in 53\% of the patients in the ERAS group. Reasons for not removing the drain on POD 4 were not fulfilling drain removal criteria or logistic problems (POD 4 was on a weekend day and drain removal was overlooked or laboratory results came back after the weekend).

In the ERAS group resumption of a solid diet on POD 2 could be achieved in $60 \%$ of the patients ( $n=52), 75 \%$ of the patients tolerated a solid diet on day POD 4 in the ERAS group ( $n=65)$. In the 'no ERAS group' patients started with intake of liquids after the NG tube was removed and started with a solid diet in the days thereafter, when liquids were tolerated.

$63 \%$ of the patients in the ERAS group $(n=54)$ were mobilized out of bed on POD 1 . In the 'no ERAS' group there was no structured mobilization.

\section{Multivariable linear regression}

The following characteristics were entered into a multivariable linear regression model: time period, age, sex, co-morbidity, indication, resection type (PPPD or classical Whipple procedure) and the presence of a complication. Multivariable regression analysis showed that the earlier time period without ERAS and the presence of complications were independent predictors of longer postoperative LOS $(P=0.003$ and $P<0.001$, respectively).

\section{DISCUSSION}

The present study shows that postoperative LOS after PD decreased significantly in the last 16 years in our institution, while morbidity, mortality and readmission rate remained unchanged. More elderly patients were selected for PD in the most recent 
period and there was an increasing trend towards operations performed for malignant disease. The improved outcomes after PD over the last decade might be attributed to the streamlining of patient care and surgical techniques. However, the implementation of an ERAS program after PD may have influenced the results, since LOS decreased dramatically from 2006 onwards. This was the time period when perioperative PD management in Maastricht was increasingly performed within an ERAS environment. The present results are in line with a previous report of Balcom et al. introducing case management and implementation of a clinical pathway in 733 cases of various types of pancreatic resection. For PD this study reported a decrease in mean LOS of 6.6 days, very similar to the reduction of LOS by 6-7 days observed for patients both with and without complications in the present study ${ }^{25}$.

Complication rates remained unchanged during the study period, except for the incidence of wound infections, which increased over the study period. However, this finding may be confounded by information bias due to the better and prospective complication registration during the final years of the study. In particular, the recording of minor complications such as would infections received more attention. Other reports on ERAS and PD also did not show an increase in morbidity after implementing an ERAS programme ${ }^{26-28}$ and one report even showed a decrease in morbidity ${ }^{29}$. The rates of PD specific complications such as DGE, pancreatic fistula and post PD hemorrhage observed in this study were comparable with other reports on fast track and PD ${ }^{29,30}$. Balzano et al. described a decrease in DGE after the institution of a fast track protocol for PD from $24.6 \%$ to $13.9 \%{ }^{29}$. In the present study, DGE did not change significantly after the implementation of an ERAS protocol (incidence of $12.8 \%$ in the ERAS group). One study reported a decrease in DGE after a clinical pathway was adopted but attributed this to the fact that more classical Whipple's procedures were performed in their clinic in the final years instead of pylorus-preserving PDs ${ }^{25}$. In the present study PPPDs were performed increasingly over the years but no significant difference was noted between the incidences of DGE in the classical Whipple-group as compared to the PPPD-group. This observation is in line with a previous report by Tran et al. ${ }^{31}$.

Over time, there was a trend for complications to be increasingly managed by percutaneous drainage instead of relaparotomy. This is probably explained by the increasing use of CT and advances in CT-guided percutaneous interventions. Relaparotomy rates decreased from $13 \%$ to $8 \%$, which corresponds with other reports $^{29-31}$. Readmission rates in the present study did not change significantly, which implies that earlier discharge in the years after implementation of the ERAS protocol did not affect patient morbidity.

It could be argued that improved outcomes in terms of reduced LOS are not the result of an ERAS protocol but instead the result of secular trends such as advanced surgical techniques and increased experience of surgeons. However, a recent report after implementation of an ERAS program in colonic surgery in over 30 hospitals in the 
Netherlands in 1 year demonstrated that LOS can be decreased by 3 days in addition to secular trends ${ }^{32}$.

Multivariable regression analysis showed that the presence of a complication was an independent predictor of increased LOS. This was expected as in these patients the ERAS program can only be followed to certain extent. Brooks et al. reviewed a case series of 215 patients undergoing PD and found that complications and also age and operative year were independently associated with postoperative LOS using multivariable analysis ${ }^{33}$. This is in accordance with the present results showing that a later time period (ERAS) was correlated with a lower LOS. In the current study the whole cohort was divided into three time periods reflecting the implementation of some ERAS elements ('ERAS-like') and the implementation of the ERAS protocol for PD (ERAS). There was no significant difference in LOS between the ERAS group and the 'ERAS like' group. This suggests that the introduction of several ERAS elements as compared to the introduction of a full ERAS protocol might be equivalent in terms of improving LOS. Ahmed et al. have shown that in colonic surgery that an ERAS protocol compliance of $77 \%$ does not worsen outcome when compared to a compliance of $88 \%$ in the trial group ${ }^{34}$. Thus, the protocol elements may not all be equally important. Supporting the aforementioned finding, another study in colonic surgery analyzing individual ERAS elements showed that early cessation of i.v. fluids, the use of laxatives and mobilization from day 1 were associated with a lower $\operatorname{LOS}^{32}$. However, the previous study was performed in patients undergoing colonic surgery and these results might not be extrapolatable to pancreatic surgery.

Furthermore, a recent retrospective study comparing fast track recovery with traditional care in patients undergoing PD without complications also showed that fast track recovery was associated with shorter $\operatorname{LOS}^{35}$. LOS without complications in the aforementioned study was 8 days in the fast track group, compared to 9 days in the our study.

Apart from the implementation of an ERAS protocol, another factor that might have contributed to improved outcome in this study was the increase in resection volume over the years at MUMC. A large population-based study of 3736 patients undergoing pancreatic resections showed that a higher hospital volume was correlated with lower mortality ${ }^{36}$. Also, the centralization of pancreatic resections, leading to an increase in hospital volume, has led to significant improvements in survival, mortality and complication rates , $^{2,37-39}$.

Although complications were prospectively registered in the final few years of the study, it is clear that this retrospective study has several limitations. A randomized controlled trial comparing ERAS with traditional care in PD would be the strongest way to assess the true effect of ERAS. However, this brings up ethical questions since the majority of ERAS protocol elements have been separately proven to be effective in RCTs and meta-analyses and several ERAS protocol elements have now become standard care. 
To conclude, the present study shows that in the authors' center LOS after PD was reduced significantly in the last 16 years without altering complication, mortality, and readmission rates, even though more elderly patients were operated on. Implementation of an ERAS protocol may have contributed to the improvement in LOS without compromising other outcomes. 


\section{REFERENCES}

1. Whipple AO, Parsons WB, Mullins CR. Treatment of Carcinoma of the Ampulla of Vater. Ann Surg 1935,102:763-779.

2. de Wilde RF, Besselink MG, van der Tweel I, de Hingh IH, van Eijck CH, Dejong CH, Porte RJ, Gouma DJ, Busch OR, Molenaar IQ; Dutch Pancreatic Cancer Group. Impact of nationwide centralization of pancreaticoduodenectomy on hospital mortality. Br J Surg 2012,99:404-410.

3. Gouma DJ, van Geenen RC, van Gulik TM, de Haan RJ, de Wit LT, Busch OR, et al. Rates of complications and death after pancreaticoduodenectomy: risk factors and the impact of hospital volume. Ann Surg 2000,232:786-795.

4. Balcom JHt, Rattner DW, Warshaw AL, Chang Y, Fernandez-del Castillo C. Ten-year experience with 733 pancreatic resections: changing indications, older patients, and decreasing length of hospitalization. Arch Surg 2001,136:391-398.

5. Kehlet H. Multimodal approach to control postoperative pathophysiology and rehabilitation. $\mathrm{Br} J$ Anaesth 1997,78:606-617.

6. Barbieri A, Vanhaecht K, Van Herck P, Sermeus W, Faggiano F, Marchisio S, et al. Effects of clinical pathways in the joint replacement: a meta-analysis. BMC Med 2009,7:32.

7. Arsalani-Zadeh R, ElFadl D, Yassin N, MacFie J. Evidence-based review of enhancing postoperative recovery after breast surgery. Br J Surg 2011,98:181-196.

8. Podore PC, Throop EB. Infrarenal aortic surgery with a 3-day hospital stay: A report on success with a clinical pathway. J Vasc Surg 1999,29:787-792.

9. Brustia P, Renghi A, Gramaglia L, Porta C, Cassatella R, De Angelis R, et al. Mininvasive abdominal aortic surgery. Early recovery and reduced hospitalization after multidisciplinary approach. J Cardiovasc Surg 2003,44:629-635.

10. McCarty TM, Arnold DT, Lamont JP, Fisher TL, Kuhn JA. Optimizing outcomes in bariatric surgery: outpatient laparoscopic gastric bypass. Ann Surg 2005,242:494-498; discussion 498-501.

11. Wasowicz-Kemps DK, Bliemer B, Boom FA, de Zwaan NM, van Ramshorst B. Laparoscopic gastric banding for morbid obesity: outpatient procedure versus overnight stay. Surg EndosC 2006,20: 1233-1237.

12. Kirsh EJ, Worwag EM, Sinner M, Chodak GW. Using outcome data and patient satisfaction surveys to develop policies regarding minimum length of hospitalization after radical prostatectomy. Urology 2000,56:101-106; discussion 106-107.

13. Coolsen MM, van Dam RM, van der Wilt AA, Slim K, Lassen K, Dejong CH. Systematic Review and Metaanalysis of Enhanced Recovery After Pancreatic Surgery with Particular Emphasis on Pancreaticoduodenectomies. World J Surg 2013,37:1909-1918.

14. di Sebastiano P, Festa L, De Bonis A, Ciuffreda A, Valvano MR, Andriulli A, et al. A modified fast-track program for pancreatic surgery: a prospective single-center experience. Langenbecks Arch Surg 2011,396:345-351.

15. Nygren J, Hausel J, Kehlet H, Revhaug A, Lassen K, Dejong C, et al. A comparison in five European Centres of case mix, clinical management and outcomes following either conventional or fast-track perioperative care in colorectal surgery. Clin Nutr 2005,24:455-461.

16. van Dam RM, Hendry PO, Coolsen MM, Bemelmans MH, Lassen K, Revhaug A, et al. Initial experience with a multimodal enhanced recovery programme in patients undergoing liver resection. $\mathrm{Br} J$ Surg 2008,95:969-975.

17. Lassen K, Coolsen MM, Slim K, Carli F, de Aguilar-Nascimento JE, Schafer M, et al. Guidelines for perioperative care for pancreaticoduodenectomy: Enhanced Recovery After Surgery (ERAS(R)) Society recommendations. Clin Nutr 2012,31:817-830.

18. Mangram AJ, Horan TC, Pearson ML, Silver LC, Jarvis WR. Guideline for Prevention of Surgical Site Infection, 1999. Centers for Disease Control and Prevention (CDC) Hospital Infection Control Practices Advisory Committee. Am J Infect Control 1999,27:97-132; quiz 133-134; discussion 196.

19. Bassi C, Dervenis C, Butturini G, Fingerhut A, Yeo C, Izbicki J, et al. Postoperative pancreatic fistula: an international study group (ISGPF) definition. Surgery 2005,138:8-13. 
20. Wente MN, Bassi C, Dervenis C, Fingerhut A, Gouma DJ, Izbicki JR, et al. Delayed gastric emptying (DGE) after pancreatic surgery: a suggested definition by the International Study Group of Pancreatic Surgery (ISGPS). Surgery 2007,142:761-768.

21. Levy MM, Fink MP, Marshall JC, Abraham E, Angus D, Cook D, et al. 2001 SCCM/ESICM/ACCP/ATS/SIS International Sepsis Definitions Conference. Intensive Care Med 2003,29:530-538.

22. Welsch T, Eisele H, Zschabitz S, Hinz U, Buchler MW, Wente MN. Critical appraisal of the International Study Group of Pancreatic Surgery (ISGPS) consensus definition of postoperative hemorrhage after pancreatoduodenectomy. Langenbecks Arch Surg 2011,396:783-791.

23. Maher JW, Bakhos W, Nahmias N, Wolfe LG, Meador JG, Baugh N, et al. Drain amylase levels are an adjunct in detection of gastrojejunostomy leaks after Roux-en-Y gastric bypass. J Am Coll Surg 2009,208:881-884; discussion 885-886.

24. Dindo D, Demartines N, Clavien PA. Classification of surgical complications: a new proposal with evaluation in a cohort of 6336 patients and results of a survey. Ann Surg 2004,240:205-213.

25. Balcom JHt, Rattner DW, Warshaw AL, Chang Y, Fernandez-del Castillo C. Ten-year experience with 733 pancreatic resections: changing indications, older patients, and decreasing length of hospitalization. Arch Surg 2001,136:391-398.

26. Kennedy EP, Rosato EL, Sauter PK, Rosenberg LM, Doria C, Marino IR, et al. Initiation of a critical pathway for pancreaticoduodenectomy at an academic institution--the first step in multidisciplinary team building. J Am Coll Surg 2007,204:917-923; discussion 923-914.

27. Porter GA, Pisters PW, Mansyur C, Bisanz A, Reyna K, Stanford P, et al. Cost and utilization impact of a clinical pathway for patients undergoing pancreaticoduodenectomy. Ann Surg Oncol 2000,7:484-489.

28. Vanounou T, Pratt W, Fischer JE, Vollmer CM, Jr., Callery MP. Deviation-based cost modeling: a novel model to evaluate the clinical and economic impact of clinical pathways. J Am Coll Surg 2007,204: 570-579.

29. Balzano G, Zerbi A, Braga M, Rocchetti S, Beneduce AA, Di Carlo V. Fast-track recovery programme after pancreatico- duodenectomy reduces delayed gastric emptying. Br J Surg 2008,95:1387-1393.

30. Robertson N, Gallacher PJ, Peel N, Garden OJ, Duxbury M, Lassen K, et al. Implementation of an enhanced recovery programme following pancreaticoduodenectomy. HPB (Oxford) 2012,14:700-708.

31. Tran KT, Smeenk HG, van Eijck CH, Kazemier G, Hop WC, Greve JW, et al. Pylorus preserving pancreaticoduodenectomy versus standard Whipple procedure: a prospective, randomized, multicenter analysis of 170 patients with pancreatic and periampullary tumors. Ann Surg 2004,240: 738-745.

32. Gillissen F, Hoff C, Maessen JM, Winkens B, Teeuwen JH, von Meyenfeldt MF, et al. Structured Synchronous Implementation of an Enhanced Recovery Program in Elective Colonic Surgery in 33 Hospitals in The Netherlands. World J Surg 2013.

33. Brooks AD, Marcus SG, Gradek C, Newman E, Shamamian P, Gouge TH, et al. Decreasing length of stay after pancreatoduodenectomy. Arch Surg 2000,135:823-830.

34. Ahmed J, Khan S, Gatt M, Kallam R, MacFie J. Compliance with enhanced recovery programmes in elective colorectal surgery. Br J Surg 2010,97:754-758.

35. Nikfarjam M, Weinberg L, Low N, Fink MA, Muralidharan V, Houli N, et al. A fast track recovery program significantly reduces hospital length of stay following uncomplicated pancreaticoduodenectomy. JOP 2013,14:63-70.

36. Riall TS. What is the effect of age on pancreatic resection? Adv Surg 2009,43:233-249.

37. Gooiker GA, van der Geest LG, Wouters MW, Vonk M, Karsten TM, Tollenaar RA, et al. Quality improvement of pancreatic surgery by centralization in the western part of the Netherlands. Ann Surg Oncol 2011,18:1821-1829.

38. Balzano G, Zerbi A, Capretti G, Rocchetti S, Capitanio V, Di Carlo V. Effect of hospital volume on outcome of pancreaticoduodenectomy in Italy. Br J Surg 2008,95:357-362.

39. Topal B, Van de Sande S, Fieuws S, Penninckx F. Effect of centralization of pancreaticoduodenectomy on nationwide hospital mortality and length of stay. Br J Surg 2007,94:1377-1381. 


\section{CHAPTER}

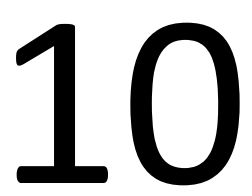

Implementing an enhanced recovery program after pancreaticoduodenectomy in elderly patients - is it feasible?

MME Coolsen, M Bakens, RM van Dam, SWM Olde Damink, CHC Dejong World J Surg. 2014 Sep 12. Epub ahead of print 


\section{ABSTRACT}

\section{Background}

An enhanced recovery after surgery (ERAS) program aims to reduce the stress response to surgery and thereby accelerate recovery. It is unclear whether these programs can be safely implemented for elderly patients, especially in highly complex surgery such as pancreaticoduodenectomy (PD). The objective of this study was to evaluate the feasibility of an ERAS program in elderly patients undergoing PD.

\section{Methods}

Implementation of the ERAS protocol was studied prospectively in a consecutive series of patients undergoing PD between January 2009 and August 2013. Patients were divided into two groups: $\leq 65$ years and $\geq 70$ years. Endpoints were length of stay (LOS), readmissions, morbidity, mortality and compliance with ERAS targets.

\section{Results}

Of a total of 110 patients, 55 were $\leq 65$ years (median 57 ) and $55 \geq 70$ years (median 77). Median LOS was 14 days in both groups. In patients without complications median LOS was 9 days. Both mortality and readmissions did not differ between groups (mortality $n=3(5.5 \%)$ in younger versus $\mathrm{n}=6(10.9 \%)$ in older patients, $P=0.49$, readmissions: $n=11(20 \%)$ versus $n=7(12.7 \%), P=0.44)$. CT-drainage and relaparotomy-rates were not different between groups, nor was overall morbidity $(n=31(56.3 \%)$ in the older versus $n=35(63.3 \%)$ in the younger group, $P=0.44)$. There were no differences in compliance with elements of the ERAS protocol between groups.

\section{Conclusion}

An ERAS program seems feasible and safe for patients $\geq 70$ years of age undergoing PD. Improving protocol compliance may further reduce morbidity and mortality, especially in high-risk patients. 


\section{INTRODUCTION}

In the Netherlands, approximately 2000 patients are affected by cancer of the pancreas and periampullary region each year. Without treatment median survival after diagnosis is only 4-6 months. Patients with potentially curable disease $( \pm 20 \%)$ may undergo surgery with curative intent, but after 'curative' resection the prognosis is still poor with 5-year survival rates of $10-20 \%{ }^{1}$. Pancreaticoduodenectomy (PD) remains the only curative option for the majority of pancreatic and periampullary tumors. With an increasingly aging population, more elderly people suffering from pancreatic cancer will be presented for PD. In the past, surgeons were reluctant to perform PD on elderly patients. Common reasons were a reduced physical fitness and increased co-morbidity. Recent reports, however, show that PD is feasible in the elderly population with acceptable mortality and morbidity rates ${ }^{2-6}$.

To diminish the surgical stress response and speed up recovery 'Enhanced Recovery After Surgery' (ERAS) or 'fast track' pathways have been implemented in several forms of surgery ${ }^{7-10}$. Avoidance of pre- and postoperative fastening, optimized analgesia and early mobilization are typical elements of the ERAS protocol, which accelerates recovery and reduces hospital costs without compromising postoperative morbidity and mortality ${ }^{11-14}$. In PD, a complex surgical procedure with high postoperative morbidity rates, ERAS protocols have also been successfully implemented ${ }^{15-17}$. Recently, perioperative guidelines for PD have been published by the ERAS group ${ }^{18}$.

When postoperative complications do occur, the elderly in particular seem to have an increased 30-day mortality risk ${ }^{19}$. Interestingly, two recent meta-analyses on ERAS in PD and colonic surgery suggest that postoperative morbidity rates may be lower in patients managed in an ERAS program ${ }^{11,12}$. Therefore, it might be beneficial to implement ERAS programs also for an older population. However, some authors raise doubts about subjecting the elderly to ERAS programs with immediate postoperative feeding and forced mobilization ${ }^{20}$.

The aim of this study was to prospectively evaluate the feasibility and safety of implementing an ERAS program in elderly patients ( $>70$ years) undergoing PD and compare compliance and outcome with patients below 65 years of age.

\section{METHODS}

\section{Patients}

Perioperative outcome data were retrieved from a database in which all patients undergoing PD in Maastricht University Medical Center in a period between January 2009 and Augustus 2013 were entered prospectively. Patients were divided into two groups: below 65 years of age and above 70 years of age. The 5 -year gap was arbitrary 
chosen to create a real difference between the older and younger group (e.g. otherwise two people who differ two days in age could be in different groups). From January 2009 onwards a multimodal ERAS program for PD was implemented in our institution and all patients undergoing PD from that date onwards were managed accordingly. There were no exclusion criteria.

\section{Perioperative management}

A detailed description of the ERAS protocol for PD is shown in Table 10.1. Preoperatively, patients were informed at the out-patient clinic about the ERAS protocol and possible postoperative complications. Before the operation, an assessment was made of ASA classification and co-morbidity. All patients received antibiotic prophylaxis and mid-thoracic epidural analgesia.

Four surgeons, all with extensive experience in PD, performed the operative procedures. Bilateral subcostal transverse abdominal incisions were used. The biliodigestive anastomosis, gastrojejunostomy and pancreaticojejunostomy were hand sewn and a prophylactic abdominal silicon drain was placed near the pancreaticojejunostomy at the end of the procedure. NG tubes were not placed or were removed after the procedure directly before transportation to the recovery ward.

Patients were allowed to restart oral intake of water on the day of surgery, to drink freely up to 1.5 I on the first postoperative day (POD) and resume their normal diet on the second postoperative day. Furthermore, patients received magnesiumoxide $1000 \mathrm{mg}$ twice daily. Mobilization out of bed was started on POD 1. Epidural analgesia was stopped on POD 3 and replaced by NSAIDs. The prophylactic abdominal drain was removed on POD 4 if amylase was less than 3 times the serum amylase concentration or drain production was less than $50 \mathrm{ml}$ per day. Patients were discharged if functional recovery criteria were met (good pain control with oral analgesia only, tolerance for solid food, no intravenous fluids, passage of stool and being independently mobile at the pre-operative level) and if the patient was willing to go home.

\section{Primary and secondary endpoints}

The primary endpoint was postoperative length of stay (LOS), defined as nights spent in hospital after the operation. Secondary endpoints were postoperative complications, mortality, readmissions, postoperative interventions and compliance with various elements of the ERAS protocol. Complications were defined as any deviation from the normal postoperative course. Definitions used for specific complications were according to literature (Appendix 5$)^{21-26}$ and further classified according to the DindoClavien classification ${ }^{27}$. Data were recorded prospectively during hospital stay and the 30-day period after surgery in an electronic registration system (SAP) as well as in a database. For mortality the follow-up was 90 days. In the morning after discharge, complications were registered in SAP for each patient. 
Table 10.1 ERAS protocol for pancreaticoduodenectomy

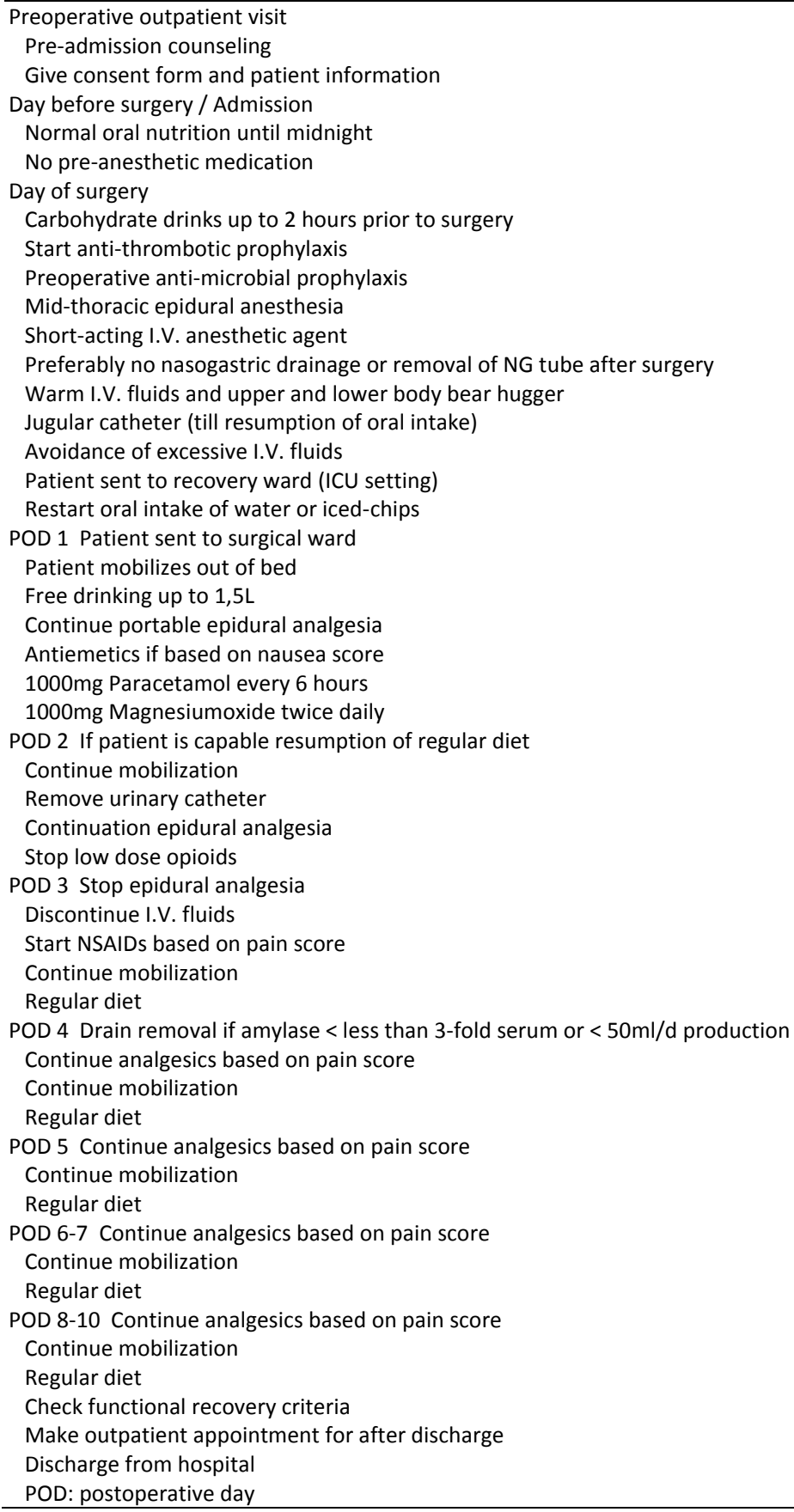


Postoperative data in SAP were also crosschecked with existing in-house databases containing prospectively collected complications. Readmissions were recorded if related to the index-operation within a time-period of 6 months.

Adherence to various ERAS protocol elements that were specifically reviewed included resumption of oral fluid and solid intake, mobilization from POD 1, drain removal on POD 4, urinary catheter removal on POD 2 and removal of epidural analgesia on POD 3.

\section{Data analysis and statistics}

Continuous data are given as median (range). Categorical variables were analyzed using the $\chi 2$ test or Fisher's exact test, quantitative variables with Student's $t$ test. Postoperative length of stay was analyzed using the non-parametric Mann-Whitney $U$ test. A p-value $<0.05$ was considered significant. Statistical analysis was performed using SPSS for Windows, version 19.

\section{RESULTS}

\section{Demographics and operative outcome}

A consecutive series of 110 patients undergoing PD between January 2009 and August 2013 was included in this study. Of these, 55 patients were below 65 years of age and 55 patients were older than 70 years (Table 10.2). The median age for the $\leq 65$ years group was 57 (range 45-65) and for the $\geq 70$ years group 77 (range 71-86). There were significantly more patients with a higher ASA classification in the older group. Furthermore, cardiac and pulmonary co-morbidity was more frequently present in the older group ( $P<0.016$ and $P<0.014$ respectively). BMI (Body Mass Index) and type of pancreatic pathology did not differ between groups. In both groups most resections were performed for malignancies and more PPPDs than classical Whipple procedures were performed ( $84 \%$ versus $16 \%$ in the younger group and $85 \%$ versus $15 \%$ in the older group). Mean operative time did not differ between groups.

\section{Postoperative outcome}

Postoperative outcomes parameters are shown in Table 10.3. Median LOS was not different between the two groups (both 14 days with ranges 6-100 and 6-65 respectively), nor was median LOS for younger and older patients discharged without complications (9 days with ranges 6-15 and 6-22 respectively). Readmissions within 6 months of the index-hospitalization were not significantly different between the two groups $(18.1 \%$ and $12.7 \%$ respectively). The same holds true for mortality rates although a trend to an increased mortality rate in the older group was observed $(5.5 \%$ and $10.9 \%$ respectively). There were no differences in postoperative intervention rate 
(CT/US-drainage and relaparotomy) and in minor (Dindo-Clavien grade I and II), moderate (grade IIla/b) or severe (grade IVa/IVb) complications between the groups. Median LOS was also calculated for Dindo-Clavien grades below and above III. Patients who suffered from complications in the elderly group had a longer median postoperative length of stay than those in the younger group for Dindo-Clavien grades $<$ III $(P=0.028)$.

Table 10.2 Patient demographics, pathology and operative data

\begin{tabular}{|c|c|c|c|}
\hline & $\begin{array}{c}\leq 65 \text { years of age } \\
(n=55)\end{array}$ & $\geq 70$ years of age $(n=55)$ & p-value \\
\hline Age median (range) & $57(45-65)$ & $77(71-86)$ & \\
\hline \multicolumn{4}{|l|}{ Sex } \\
\hline Male/female & $35 / 20$ & $22 / 33$ & 0.013 \\
\hline \multicolumn{4}{|l|}{ ASA class } \\
\hline 1 & $28(51 \%)$ & $1(2 \%)$ & $>0.001 *$ \\
\hline II & $21(38 \%)$ & $34(62 \%)$ & 0.013 \\
\hline III & $5(9 \%)$ & $20(36 \%)$ & $0.001^{*}$ \\
\hline IV & $1(2 \%)$ & $0(0 \%)$ & $0.315^{*}$ \\
\hline \multicolumn{4}{|l|}{ Comorbidity } \\
\hline Cardiovascular & $13(24 \%)$ & $25(45 \%)$ & 0.016 \\
\hline Pulmonary & $2(4 \%)$ & $10(18 \%)$ & $0.014^{*}$ \\
\hline Diabetes & $6(11 \%)$ & $12(21.8 \%)$ & $0.122 *$ \\
\hline Renal disease & $1(2 \%)$ & $6(10.9 \%)$ & $0.056^{*}$ \\
\hline BMI (range) & $24.9(18.0-36.1)$ & $24.4(18.6-36.8)$ & 0.656 \\
\hline \multicolumn{4}{|l|}{ Pathology } \\
\hline Pancreatic head carcinoma & $34(62 \%)$ & $33(60 \%)$ & 0.845 \\
\hline Duodenal cancer & $2(4 \%)$ & $3(5 \%)$ & $1.000 *$ \\
\hline Ampullary carcinoma & $8(15 \%)$ & $12(22 \%)$ & $0.459 *$ \\
\hline Distal cholangiocarcinoma & $5(9 \%)$ & $5(9 \%)$ & $1.000 *$ \\
\hline Metastasis in head of pancreas & $1(1,8 \%)$ & $0(0 \%)$ & $1.000^{*}$ \\
\hline Chronic pancreatitis & $2(4 \%)$ & $2(4 \%)$ & $1.000 *$ \\
\hline IPMN & $1(2 \%)$ & $0(0 \%)$ & $1.000 *$ \\
\hline Neuro-endocrine tumor & $2(4 \%)$ & $0(0 \%)$ & $0.495^{*}$ \\
\hline \multicolumn{4}{|l|}{ Operation type } \\
\hline Whipple & $9(16 \%)$ & $8(15 \%)$ & 1.000 \\
\hline$P P P D$ & $46(84 \%)$ & 47 (85\%) & 0.792 \\
\hline Duration of OR time (min) & $345(180-810)$ & $340(217-715)$ & $0.986^{\dagger}$ \\
\hline
\end{tabular}

ASA: American Society of Anesthesiologists, BMI: Body Mass Index, PPPD: pylorus preserving pancreaticoduodenectomy. All statistics were performed with Chi-square, marked * with the Fisher's exact test, marked ${ }^{\dagger}$ with T-test. 
Table 10.3 Postoperative outcomes

\begin{tabular}{lccc}
\hline & $\begin{array}{c}\leq 65 \text { years of age } \\
(\mathrm{n}=55)\end{array}$ & $\begin{array}{c}\geq 70 \text { years of age } \\
(\mathrm{n}=55)\end{array}$ & P-value \\
\hline Dindo-Clavien classification & $15(27.2 \%)$ & $20(36.3 \%)$ & 0.409 \\
$\quad$ Minor (I/II) & $15(27.2 \%)$ & $8(14.5 \%)$ & $0.159^{*}$ \\
Moderate (IIIa/b) & $0(0 \%)$ & $2(3.6 \%)$ & $0.495^{*}$ \\
Severe (Iva en IVb) & $3(5,5 \%)$ & $6(10.9 \%)$ & $0.489^{*}$ \\
Mortality < 90days & $11(20 \%)$ & $7(12.7 \%)$ & $0.440^{*}$ \\
Readmissions < 6 months & $14(6-100)$ & $14(6-65)$ & 0.186 \\
Median length of hospital stay (range) & $9(6-15)$ & $9(6-22)$ & 0.275 \\
Length of stay uncomplicated (median) & $10(6-100)$ & $14(7-59)$ & 0.028 \\
Median LOS Dindo-Clavien grade <III (range) & $32(11-83)$ & $33(14-65)$ & 0.488 \\
Median LOS Dindo-Clavien grade >III (range) & & & $0.797^{*}$ \\
Interventions & $10(18.1 \%)$ & $8(14.5 \%)$ & $0.742^{*}$ \\
CT/US-drainage & $6(10.9 \%)$ & $4(7.2 \%)$ & \\
Re-operation & & & \\
\hline
\end{tabular}

LOS: Mann Whitney U. *Fisher exact, all other statistics: Chi Square

\section{Morbidity}

Table 10.4 summarizes the overall and specific complications in both groups. Overall morbidity was $56.3 \%$ in the $\leq 65$ years group and $63.3 \%$ in the $\geq 70$ years group ( $P=n s$ ). The incidence of specific complications did not differ between groups.

Table 10.4 Complications specified

\begin{tabular}{lccc}
\hline & $\begin{array}{c}\text { s6 years of age } \\
(\mathrm{n}=55)\end{array}$ & $\begin{array}{c}\geq 70 \text { years of age } \\
(\mathrm{n}=55)\end{array}$ & P-value \\
\hline Overall complications & $31(56.3 \%)$ & $35(63.3 \%)$ & $0.436^{*}$ \\
Intra abdominal abscess & $7(13 \%)$ & $7(13 \%)$ & 1.000 \\
Wound infection & $7(13 \%)$ & $7(13 \%)$ & 1.000 \\
Pancreatic fistula & $9(16 \%)$ & $5(9 \%)$ & 0.392 \\
Pneumonia & $2(3,6 \%)$ & $2(3,6 \%)$ & 1.000 \\
Delayed gastric emptying grade & $5(9 \%)$ & $5(9 \%)$ & 1.000 \\
Biliodigestive anastomosis leakage & $3(6 \%)$ & $1(2 \%)$ & 0.618 \\
Sepsis/MOF & $2(4 \%)$ & $6(11 \%)$ & 0.271 \\
Post pancreaticoduodenectomy hemorrhage & $5(9 \%)$ & $5(9 \%)$ & 1.000 \\
Urinary tract infection & $3(6 \%)$ & $5(9 \%)$ & 0.761 \\
Gastrojejunostomy leak & $1(2 \%)$ & $0(0 \%)$ & 0.850 \\
Chylus leakage & $0(0 \%)$ & $2(4 \%)$ & 0.495 \\
Diabetes mellitus de novo & $1(1,8 \%)$ & $3(6 \%)$ & 0.618 \\
CVA & $0(0 \%)$ & $1(2 \%)$ & 0.850 \\
Cardiac & $6(11 \%)$ & $9(16 \%)$ & 0.580 \\
\hline
\end{tabular}

All statistics with fisher's exact test. ${ }^{*}$ Chi-square 


\section{ERAS outcomes}

NG tubes were either not placed or directly removed after surgery in all patients. As Table 10.5 indicates compliance with resumption of oral fluid intake on POD 1 was high in both groups ( $96 \%$ in the younger and $95 \%$ in the older group). Solid oral intake was started from POD 2 onwards in 56\% of patients in the younger group and $51 \%$ of patients in the older group ( $P=\mathrm{ns})$.

Table 10.5 ERAS outcomes

\begin{tabular}{lccc}
\hline & $\begin{array}{c}\leq 65 \text { years of age } \\
(\mathrm{n}=55)\end{array}$ & $\begin{array}{c}\geq 70 \text { years of age } \\
(\mathrm{n}=55)\end{array}$ & $\mathrm{p}$-value \\
\hline Resumption of fluid intake POD 1 & $53(96 \%)$ & $52(95 \%)$ & 0.647 \\
Resumption of solid intake POD 2 & $31(56 \%)$ & $35(63 \%)$ & 0.436 \\
Mobilization from POD 1 & $33(60 \%)$ & $35(63 \%)$ & 0.695 \\
Drain removal on POD 4 & $26(47 \%)$ & $28(51 \%)$ & 0.703 \\
Urinary catheter removal on POD 2 & $38(69 \%)$ & $33(60 \%)$ & 0.319 \\
Removal epidural on POD 3 & $44(80 \%)$ & $43(78 \%)$ & 0.815 \\
\hline
\end{tabular}

All statistics were performed with Chi-square

Reasons for not starting solid intake were nausea and malaise. Mobilization on POD 1 was $60 \%$ in the younger and $63 \%$ in the older group. A lower compliance with mobilization was mainly due to weakness, hypotension or pain. Prophylactic abdominal drains were removed in $47 \%$ of the patients on POD 4 in the younger group and $51 \%$ in the older group $(p=n s)$. In half of the patients the drain was not removed on POD 4 because the drain removal criteria were not met. In the other half this was due to logistic problems (e.g. POD 4 fell on a weekend day and drain removal was overlooked or laboratory results came in later). The number of patients having their urinary catheter removed on POD 2 was lower than expected. This was mainly due to the fact that the epidural catheter was removed on POD 3 in a considerable number of patients. Consequently, urinary catheters were often removed only after epidural analgesia was stopped. Also, some patients needed longer catheterization because of bladder problems or in order to monitor urine output in critically ill patients.

For completeness, the results of the 25 patients in the age-group that was left out (66-69 years) are presented in Appendix 8 and 9. These are similar to the results of both other groups.

\section{DISCUSSION}

This study describes the results of the implementation of an ERAS program in PD in elderly patients. It compares results in patients $\leq 65$ years of age with those in patients 
$\geq 70$ years. Implementing an ERAS program for PD in the elderly appeared to be feasible since compliance with the different ERAS protocol elements was similar in younger and older patients. Its safety also seems confirmed since readmission, morbidity and mortality rates did not differ significantly between the two groups.

Several studies have evaluated risks and outcomes after PD in the elderly and have shown that it is possible to perform PD with acceptable morbidity and mortality rates $^{2,5}$. However, overall morbidity rates vary greatly between single-center studies $(21-73 \%)^{28}$. The variance may not only reflect differences in outcome between highand low-volume HPB centers and registration flaws in retrospective studies, but also failure to use a standardized complication registration system and unanimous definitions for specific complications. In this study universal definitions for complications were used and complications were classified according to DindoClavien ${ }^{27}$. Overall morbidity in the present study was roughly compared to the morbidity reported in a recent case series involving 2698 patients undergoing PD (42\% $<80$ years and $53 \% 80-89$ years $)^{29}$. The slightly higher morbidity in the present study might be due to the meticulous prospective complication registration in the present study. Some studies report higher incidences of pancreatic fistula (PF) and DGE after PD in the elderly, but the current study did not confirm this ${ }^{2,430}$. In line with previous reports, the mortality rate we recorded, though not significantly, tended to be higher in the older group than in the younger group $(10.9 \%$ and $5.5 \%$ respectively, $P=0.489)^{28,30-32}$. The mortality rate for the elderly as shown in our study was at the high end of the range reported in studies with similar sample sizes (mortality $0-10.5 \%)^{3,4,30,32,33}$. However, not all these studies defined mortality in an exact manner or restricted themselves to 30-day mortality rates, compared to a 90-day mortality defined in this study. Moreover, two population-based studies including 23518 and 3736 patients undergoing pancreatic resection showed even higher mortality rates in the elderly population (up to $15.5 \%$ and $11.4 \%$ respectively) ${ }^{34,35}$. This was also confirmed in a Dutch study including 2155 PD's $(10.4 \%$ in patients $>70$ compared to $4.4 \%$ in the younger group) ${ }^{36}$.

Planned discharge in this study was on POD 8-10. This target appeared to be feasible only in patients without postoperative complications (median discharge at day 9). The overall median length of stay was 14 days. This was probably due to the fact that more than half of the patients had complications. Another explanation of the relatively high median LOS might be the fact that physicians are reluctant to discharge PD patients early after surgery, since major complications can even occur with some delay after surgery. In a recent study in patients undergoing colonic surgery, only half of the patients were discharged when all discharge criteria were met ${ }^{37}$, and $43 \%$ of this group was kept in hospital because of surgeons' judgment.

The reported length of stay in the present study is in line with other studies on reporting a postoperative length of stay after PD of approximately 13 days in the ERAS 
group $^{15,16}$. In other studies on PD in elderly with traditional care median length of stay varied from 13-25 days in the older population ${ }^{34,35,38}$.

Overall compliance with the various ERAS elements was acceptable (73\%). Ahmed et al. already showed that an ERAS protocol compliance of more than $77 \%$ does not further improve outcome in colorectal surgery ${ }^{39}$. Removal of NG tubes and epidural analgesia and resumption of fluid intake were achieved for the majority of patients. However, compliance with other ERAS elements such as mobilization on POD 1, removal of urinary catheters on POD 2 and removal of prophylactic intra-abdominal drains was lower. There is still considerable debate on if and when drains should be placed and removed $^{40-43}$. Early drain removal $(<72 \mathrm{~h})$ may be advised for patients with a low risk of $\mathrm{PF}^{18}$.

There is no official definition for the term "elderly", but most studies performed on pancreatic resections in the elderly population use a cut-off age above 70 years ${ }^{4,32,33,44-46}$. A recent analysis on a large cohort showed an age of 68.5 years can be considered as 'surgical oldness' ${ }^{47}$. We decided to define an interval centered on this age and considered older than 70 years as "elderly" and below 65 years as younger patients.

Resection is the sole curative option for patients with malignant disease of the pancreas. Also in the elderly the survival benefit after resection is not diminished ${ }^{34}$. It is nevertheless crucial to carefully select older patients using meticulous pre-operative assessment $^{48}$. Better selection of patients might also contribute to lowering mortality rates. Importantly, older patients should be well informed about the risks and the fact that hospital volume and surgeon experience play a significant role in the outcome $\mathrm{e}^{35,49,50}$.

To conclude, this study evaluated the results of implementation of an ERAS program in elderly patients ( $\geq 70$ years) undergoing PD as compared to patients below 65 years of age. It shows that an ERAS program for the elderly is feasible and it can be safely implemented with comparable readmission, morbidity and mortality rates. Compliance to protocol was similar in both groups. However, efforts must be made to improve compliance with specific protocol elements to further reduce morbidity and mortality, especially in high-risk patients. 


\section{REFERENCES}

1. Centre DC. National guidlines "pancreatic carcinoma" version 2.0. Utrecht, 2011.

2. Kow AW, Sadayan NA, Ernest A, Wang B, Chan CY, Ho CK, Liau KH. Is pancreaticoduodenectomy justified in elderly patients? Surgeon 2012;10:128-36.

3. Ito $\mathrm{Y}$, Kenmochi T, Irino T, Egawa T, Hayashi S, Nagashima A, Kitagawa $\mathrm{Y}$. The impact of surgical outcome after pancreaticoduodenectomy in elderly patients. World J Surg Oncol 2011;9:102.

4. Scurtu R, Bachellier P, Oussoultzoglou E, Rosso E, Maroni R, Jaeck D. Outcome after pancreaticoduodenectomy for cancer in elderly patients. J Gastrointest Surg 2006;10:813-822.

5. Tani M, Kawai M, Hirono S, Ina S, Miyazawa M, Nishioka R, Shimizu A, Uchiyama K, Yamaue H. A pancreaticoduodenectomy is acceptable for periampullary tumors in the elderly, even in patients over 80 years of age. J Hepatobiliary Pancreat Surg 2009;16:675-680.

6. Suzuki S, Kaji S, Koike N, Harada N, Suzuki M. Pancreaticoduodenectomy can be safely performed in the elderly. Surg Today 2013;43:620-624.

7. Barbieri A, Vanhaecht K, Van Herck P, Sermeus W, Faggiano F, Marchisio S, Panella M. Effects of clinical pathways in the joint replacement: a meta-analysis. BMC Med 2009;7:32.

8. Podore PC, Throop EB. Infrarenal aortic surgery with a 3-day hospital stay: A report on success with a clinical pathway. J Vasc Surg 1999;29:787-792.

9. Arsalani-Zadeh R, ElFadl D, Yassin N, MacFie J. Evidence-based review of enhancing postoperative recovery after breast surgery. Br J Surg 2011;98:181-196.

10. Basse L, Hjort Jakobsen D, Billesbølle P, Werner M, Kehlet $\mathrm{H}$. A clinical pathway to accelerate recovery after colonic resection. Ann Surg 2000;232:51-57.

11. Varadhan KK, Neal KR, Dejong CH, Fearon KC, Ljungqvist O, Lobo DN. The enhanced recovery after surgery (ERAS) pathway for patients undergoing major elective open colorectal surgery: a meta-analysis of randomized controlled trials. Clin Nutr 2010;29:434-440.

12. Coolsen MM, van Dam RM, van der Wilt AA, Slim K, Lassen K, Dejong CH. Systematic review and metaanalysis of enhanced recovery after pancreatic surgery with particular emphasis on pancreaticoduodenectomies. World J Surg 2013;37:1909-1918.

13. Spelt L, Ansari D, Sturesson C, Tingstedt B, Andersson R. Fast-track programmes for hepatopancreatic resections: where do we stand? HPB (Oxford) 2011;13:833-838.

14. Wind J, Polle SW, Fung Kon Jin PH, Dejong CH, von Meyenfeldt MF, Ubbink DT, Gouma DJ, Bemelman WA; Laparoscopy and/or fast track multimodal management versus standard care (LAFA) study group; Enhanced recovery after surgery (ERAS) group. Systematic review of enhanced recovery programmes in colonic surgery. Br J Surg 2006;93:800-809.

15. Izano G, Zerbi A, Braga M, Rocchetti S, Beneduce AA, Di Carlo V. Fast-track recovery programme after pancreatico- duodenectomy reduces delayed gastric emptying. Br J Surg 2008;95:1387-1393.

16. Porter GA, Pisters PW, Mansyur C, Bisanz A, Reyna K, Stanford P, Lee JE, Evans DB. Cost and utilization impact of a clinical pathway for patients undergoing pancreaticoduodenectomy. Ann Surg Oncol 2000;7:484-489.

17. di Sebastiano P, Festa L, De Bonis A, Ciuffreda A, Valvano MR, Andriulli A, di Mola FF. A modified fasttrack program for pancreatic surgery: a prospective single-center experience. Langenbecks Arch Surg 2011;396:345-351.

18. Lassen K, Coolsen MM, Slim K, Carli F, de Aguilar-Nascimento JE, Schäfer M, Parks RW, Fearon KC, Lobo DN, Demartines N, Braga M, Ljungqvist O, Dejong CH; ERAS ${ }^{\circledR}$ Society; European Society for Clinical Nutrition and Metabolism; International Association for Surgical Metabolism and Nutrition. Guidelines for perioperative care for pancreaticoduodenectomy: Enhanced Recovery After Surgery (ERAS(R)) Society recommendations. Clin Nutr 2012;31:817-830.

19. Hamel MB, Henderson WG, Khuri SF, Daley J. Surgical outcomes for patients aged 80 and older: morbidity and mortality from major noncardiac surgery. J Am Geriatr Soc 2005;53:424-429.

20. Gouvas N, Tan E, Windsor A, Xynos E, Tekkis PP. Fast-track vs standard care in colorectal surgery: a meta-analysis update. Int J Colorectal Dis 2009;24:1119-1131. 
21. Mangram AJ, Horan TC, Pearson ML, Silver LC, Jarvis WR. Guideline for Prevention of Surgical Site Infection, 1999. Centers for Disease Control and Prevention (CDC) Hospital Infection Control Practices Advisory Committee. Am J Infect Control 1999;27:97-132.

22. Bassi C, Dervenis C, Butturini G, Fingerhut A, Yeo C, Izbicki J, Neoptolemos J, Sarr M, Traverso W, Buchler M; International Study Group on Pancreatic Fistula Definition. Postoperative pancreatic fistula: an international study group (ISGPF) definition. Surgery 2005;138:8-13.

23. Wente MN, Bassi C, Dervenis C, Fingerhut A, Gouma DJ, Izbicki JR, Neoptolemos JP, Padbury RT, Sarr MG, Traverso LW, Yeo CJ, Büchler MW. Delayed gastric emptying (DGE) after pancreatic surgery: a suggested definition by the International Study Group of Pancreatic Surgery (ISGPS). Surgery 2007; 142:761-768.

24. Levy MM, Fink MP, Marshall JC, Abraham E, Angus D, Cook D, Cohen J, Opal SM, Vincent JL, Ramsay G; International Sepsis Definitions Conference. 2001 SCCM/ESICM/ACCP/ATS/SIS International Sepsis Definitions Conference. Intensive Care Med 2003;29:530-538.

25. Welsch T, Eisele H, Zschäbitz S, Hinz U, Büchler MW, Wente MN. Critical appraisal of the International Study Group of Pancreatic Surgery (ISGPS) consensus definition of postoperative hemorrhage after pancreatoduodenectomy. Langenbecks Arch Surg 2011;396:783-791.

26. Maher JW, Bakhos W, Nahmias N, Wolfe LG, Meador JG, Baugh N, Kellum JM. Drain amylase levels are an adjunct in detection of gastrojejunostomy leaks after Roux-en-Y gastric bypass. J Am Coll Surg 2009;208:881-884; discussion 885-886.

27. Dindo D, Demartines N, Clavien PA. Classification of surgical complications: a new proposal with evaluation in a cohort of 6336 patients and results of a survey. Ann Surg 2004;240:205-213.

28. Sukharamwala P, Thoens J, Szuchmacher M, Smith J, DeVito P. Advanced age is a risk factor for postoperative complications and mortality after a pancreaticoduodenectomy: a meta-analysis and systematic review. HPB (Oxford) 2012;14:649-657.

29. Makary MA, Winter JM, Cameron JL, Campbell KA, Chang D, Cunningham SC, Riall TS, Yeo CJ. Pancreaticoduodenectomy in the very elderly. J Gastrointest Surg 2006;10:347-356.

30. Sohn TA, Yeo CJ, Cameron JL, Lillemoe KD, Talamini MA, Hruban RH, Sauter PK, Coleman J, Ord SE, Grochow LB, Abrams RA, Pitt HA. Should pancreaticoduodenectomy be performed in octogenarians? J Gastrointest Surg 1998;2:207-216.

31. Bathe OF, Levi D, Caldera H, Franceschi D, Raez L, Patel A, Raub WA Jr, Benedetto P, Reddy R, Hutson D, Sleeman D, Livingstone AS, Levi JU. Radical resection of periampullary tumors in the elderly: evaluation of long-term results. World J Surg 2000;24:353-358.

32. Brozzetti S, Mazzoni G, Miccini M, Puma F, De Angelis M, Cassini D, Bettelli E, Tocchi A, Cavallaro A. Surgical treatment of pancreatic head carcinoma in elderly patients. Arch Surg 2006;141:137-142.

33. Hodul P, Tansey J, Golts E, Oh D, Pickleman J, Aranha GV. Age is not a contraindication to pancreaticoduodenectomy. Am Surg 2001;67:270-275; discussion 275-276.

34. Finlayson E, Fan Z, Birkmeyer JD. Outcomes in octogenarians undergoing high-risk cancer operation: a national study. J Am Coll Surg 2007;205:729-734.

35. Riall TS, Reddy DM, Nealon WH, Goodwin JS. The effect of age on short-term outcomes after pancreatic resection: a population-based study. Ann Surg 2008;248:459-467.

36. de Wilde RF, Besselink MG, van der Tweel I, de Hingh IH, van Eijck CH, Dejong CH, Porte RJ, Gouma DJ, Busch OR, Molenaar IQ; Dutch Pancreatic Cancer Group. Impact of nationwide centralization of pancreaticoduodenectomy on hospital mortality. Br J Surg 2012;99:404-410.

37. Fiore JF Jr, Faragher IG, Bialocerkowski A, Browning L, Denehy L. Time to readiness for discharge is a valid and reliable measure of short-term recovery after colorectal surgery. World J Surg 2013;37: 2927-2934.

38. Riall TS. What is the effect of age on pancreatic resection? Adv Surg 2009;43:233-249.

39. Ahmed J, Khan S, Gatt M, Kallam R, MacFie J. Compliance with enhanced recovery programmes in elective colorectal surgery. Br J Surg 2010;97:754-758.

40. Bassi C, Molinari E, Malleo G, Crippa S, Butturini G, Salvia R, Talamini G, Pederzoli P. Early versus late drain removal after standard pancreatic resections: results of a prospective randomized trial. Ann Surg 2010;252:207-214. 
41. Kawai M, Tani M, Terasawa H, Ina S, Hirono S, Nishioka R, Miyazawa M, Uchiyama K, Yamaue H. Early removal of prophylactic drains reduces the risk of intra-abdominal infections in patients with pancreatic head resection: prospective study for 104 consecutive patients. Ann Surg 2006;244:1-7.

42. Conlon KC, Labow D, Leung D, Smith A, Jarnagin W, Coit DG, Merchant N, Brennan MF. Prospective randomized clinical trial of the value of intraperitoneal drainage after pancreatic resection. Ann Surg 2001;234:487-493.

43. Van Buren $\mathrm{G}$ 2nd, Bloomston M, Hughes SJ, Winter J, Behrman SW, Zyromski NJ, Vollmer C, Velanovich V, Riall T, Muscarella P, Trevino J, Nakeeb A, Schmidt CM, Behrns K, Ellison EC, Barakat O, Perry KA, Drebin J, House M, Abdel-Misih S, Silberfein EJ, Goldin S, Brown K, Mohammed S, Hodges SE, McElhany A, Issazadeh $M$, Jo $E$, Mo $Q$, Fisher WE. A randomized prospective multicenter trial of pancreaticoduodenectomy with and without routine intraperitoneal drainage. Ann Surg 2014;259: 605-612.

44. al-Sharaf K, Andren-Sandberg A, Ihse I. Subtotal pancreatectomy for cancer can be safe in the elderly. Eur J Surg 1999;165:230-235.

45. Fong Y, Blumgart LH, Fortner JG, Brennan MF. Pancreatic or liver resection for malignancy is safe and effective for the elderly. Ann Surg 1995;222:426-434.

46. Bottger TC, Engelmann R, Junginger T. Is age a risk factor for major pancreatic surgery? An analysis of 300 resections. Hepatogastroenterology 1999;46:2589-2598.

47. Kurian AA, Wang L, Grunkemeier G, Bhayani NH, Swanström LL. Defining "The Elderly" Undergoing Major Gastrointestinal Resections: Receiver Operating Characteristic Analysis of a Large ACS-NSQIP Cohort. Ann Surg 2013; 258:483-489.

48. Junejo MA, Mason JM, Sheen AJ, Moore J, Foster P, Atkinson D, Parker MJ, Siriwardena AK. Cardiopulmonary exercise testing for preoperative risk assessment before hepatic resection. Br J Surg 2012;99:1097-1104.

49. Gouma DJ, van Geenen RC, van Gulik TM, de Haan RJ, de Wit LT, Busch OR, Obertop H. Rates of complications and death after pancreaticoduodenectomy: risk factors and the impact of hospital volume. Ann Surg 2000;232:786-795.

50. Balzano G, Zerbi A, Capretti G, Rocchetti S, Capitanio V, Di Carlo V. Effect of hospital volume on outcome of pancreaticoduodenectomy in Italy. Br J Surg 2008;95:357-362. 


\section{CHAPTER 11}

Attitudes of patients and care providers to enhanced recovery after surgery programs following major abdominal surgery

M Hughes, MME Coolsen, EK Aahlin, EM Harrison, SJ McNally ${ }^{1}$ CHC Dejong, K Lassen, SJ Wigmore

The Journal of surgical research 2014 Jun 23. Epub ahead of print 


\section{ABSTRACT}

\section{Importance}

Enhanced recovery after surgery (ERAS) is a well-established pathway of perioperative care in surgery in an increasing number of specialties. In order to implement protocols and maintain high levels of compliance, continued support from care providers and patients is vital.

\section{Objective}

To assess the perceptions of care providers and patients of the relevance and importance of the ERAS targets and strategies.

\section{Design}

Multicenter cross sectional survey.

\section{Setting}

Three northern European tertiary referral centers.

\section{Participants}

109 patients and 57 care providers were surveyed.

\section{Exposure}

Pre-operative and post-operative surveys were completed by patients who underwent major hepatic, colorectal or esophagogastric surgery in these institutions. Anonymous web-based and paper surveys were sent to surgeons, anesthetists and nurses experienced in delivering enhanced recovery protocols. Each questionnaire asked the responder to rate a selection of enhanced recovery targets and strategies in terms of perceived importance.

\section{Main outcome measure}

Patient and care provider evaluation of enhanced recovery components.

\section{Results}

109 patients and 57 care providers completed the pre-operative survey. Overall both patients and care providers rated found the majority of items as important and supported ERAS principles. Being free from nausea (median 10, IQR 8-10) and being free from pain at rest (median 10, IQR 810) were rated highest by the patients. Care providers also rated these two items the highest. Early return of bowel function (median 7, IQR 5-8) and avoiding pre-anesthetic sedation (median 6, IQR 3.75-8) were scored the lowest by care providers.

\section{Conclusion and relevance}

ERAS principles are supported by both patients and care providers. This is important when attempting to implement and maintain an ERAS program. Controversies still remain regarding the relative importance of individual ERAS components. 


\section{INTRODUCTION}

Enhanced recovery after surgery (ERAS) protocols have become established practice in patients undergoing major surgery ${ }^{1}$. The original success in colorectal surgery has been followed by its application in other fields including hepatobiliary ${ }^{2}, \operatorname{vascular}^{3}$ and orthopedic surgery ${ }^{4}$.

The main measurements of success of enhanced recovery programs have been the reduced peri-operative morbidity and mortality and reduced post-operative length of hospital stay ${ }^{1}$.

However, deviation from ERAS protocols is commonly reported and this is associated with prolonged length of stay ${ }^{5}$. As well as this, barriers to implementing fast track protocols are commonly encountered ${ }^{6}$, with reluctance by care providers to accept care strategies that differ from personally preferred practice being a major factor ${ }^{7}$.

Patient acceptance is vital when attempting to achieve successful results with enhanced recovery approaches. Moreover, care provider support for enhanced recovery care components is critical in order to successfully implement post-operative care pathways relying on multi-disciplinary team input ${ }^{7}$.

It is therefore necessary to explore the views of both patients and care providers regarding their personal priorities pertaining to recovery and the favored strategies used to achieve these aims. This information is crucial to determine whether enhanced recovery programs have the correct patient-centered approach to post-operative recovery and the appropriate support of care providers in order to optimize implementation.

This survey of patients and healthcare professionals was performed to investigate these views and provide clarification of patient and clinician care priorities.

\section{METHODS}

Following satisfying the requirements of the respective institutional review boards a survey was carried out by the investigators across three Northern-European centers Edinburgh (UK), Troms $\varnothing$ (Norway) and Maastricht (The Netherlands). These institutions were selected as they represent the home institutions of the collaborating authors. They are high volume tertiary referral centers experienced in delivering ERAS protocols in hepatic, colorectal and esophagogastric surgery.

The authors developed a questionnaire for the purpose of this survey. The questionnaire aimed to quantify the responder's perception of the importance of individual enhanced recovery outcomes and strategies.

The questionnaire was divided into two sections. The first assessed individual targets to be achieved during recovery following abdominal surgery (questions 1-8). These incorporated the major domains of ERAS principles ${ }^{8}$ - pain control, gut restitution, 
mobility, overall function and hospital discharge. These items were identical for questionnaires given to care providers and patients. The second section assessed strategies on how to achieve the recovery targets specified in section 1 . The items chosen reflected common strategies utilized in enhanced recovery protocols as advocated by the best available evidence ${ }^{8}$. The patients were given four questions and the care providers were given 13 different questions relating to strategy (Appendices 10 and 11).

The questions were formulated in English and then translated into Dutch and Norwegian. A further separate translation of the questions back into English was performed to ensure accurate translation. The questionnaire was trialed locally to ensure satisfactory comprehension by responders.

Responders in each institution were given a standardized verbal explanation as to what the survey entailed and advice regarding how to complete the survey. They were asked to rate each component from 0-10 on an 11 point Likert scale, depending on how important they believed each component was. The scale used indicator statements of "not important" and "very important" at the relevant extremes of the scale to assist with scoring. An example was performed by the investigator with each responder to ensure comprehension and then the patient was left to complete the questionnaire unaided.

The survey was conducted between November 2012 and November 2013. Consecutive patients scheduled for hepatic, colorectal or esophagogastric surgery were approached and asked to complete a questionnaire on the morning of their operation or during outpatient work-up prior to surgery. This was repeated following surgery when the patient returned to the out-patient clinic two to four weeks later. Due to the exploratory nature of this survey a sample size calculation was not performed. However, it was determined that each center would recruit a minimum of 35 patients to complete the questionnaire before and after surgery. The exclusion criteria were an inability to comprehend the survey or unwillingness to participate.

A random sample of senior surgeons, anesthetists and nurses working in the centers involved in the care of these patient groups were also surveyed. This questionnaire was administered using an internet-based tool (Survey Monkey, Palo Alto, US; for Troms $\varnothing$ : Questback ${ }^{\circledR}$ ) or an identical paper-based version depending on convenience.

Results were collated and analyzed with Excel 2010 (Microsoft Corp., Seattle, US) and presented as median and interquartile range (IQR). Statistical analysis was performed with R (R Foundation for Statistical Computing, version 2.9.0). Discrete variables were compared with Fischer's exact or Chi-square tests where appropriate. Continuous data were assessed with Mann Whitney $U$ test. Scores between care-provider specialties were compared with the Kruskal-Wallis test. Statistically significant differences between pre-operative and post-operative patient scores were assessed by the Wilcoxon signed-rank test. Significance tests were adjusted for multiple comparisons using the Bonferroni correction. 
As we had devised a novel questionnaire, we undertook validation of the instrument. High internal consistency would be expected if responders scored items within the two sections (outcomes and strategies) similarly. Internal consistency of questionnaire components was determined with Cronbach's alpha including 95\% bootstrapped confidence intervals. Cronbach's alpha increases with greater inter-correlation of questionnaire components and can be interpreted as an overall measure of internal consistency.

Exploratory factor analysis was performed to assess the underlying structure of the questionnaire. The questionnaire had two sections, "attitudes to outcomes" and "attitudes to strategies". It might be expected that answers to questions assessing each of these domains would be correlated. The factor analysis examines whether questions might cluster into alternative groupings representing different underlying concepts. Principal component analysis was performed and eigenvalues generated (representing the proportion of the variance explained by each additional new factor). Eigenvalues were plotted on a scree plot and a cut-off determined. Maximum likelihood factor analysis was then performed with varimax rotation retaining the appropriate number of factors.

\section{RESULTS}

\section{Demographics}

One hundred and nine patients scheduled for major abdominal surgery were approached and participated in the pre-operative survey (100\% response). Patients were included from all three centers - Edinburgh $(n=38)$, Troms $\varnothing(n=36)$ and Maastricht $(n=35)$. The demographic and clinical characteristics of the patients are shown in Table 11.1. Eighty-one per cent (88/109) of patients responded to the follow-up survey administered after surgery. The post-operative non-responders had higher ICU admission rates $(29 \%$ vs. $8 \%, P<0.05)$ and a higher proportion of esophagogastric resections $(24 \%$ vs. $7 \% \quad P<0.05)$ performed when compared to the patients who completed the post-operative questionnaire. Sixteen anesthetists (median age 51, IQR 42-61), 23 nurses (median age 35, IQR 29-38) and 18 surgeons (median age 52, IQR 43-59) were surveyed from across all three sites.

\section{Questionnaire validation}

The inter-correlation of items within the two parts of the questionnaire was good suggesting internal consistency: care providers outcomes $\alpha=0.89$ (0.83-0.93), care providers strategies $\alpha=0.83$ (0.75-0.89); patients outcomes $\alpha=0.96$ (0.79-1.00.), patient strategies $\alpha=1.00(0.34-1.00)$. 
An exploratory factor analysis was performed on the questionnaire components for care providers to assess underlying structure. Three factors were shown to provide an adequate fit. Items relating mainly to enhanced recovery outcomes loaded onto one factor and those relating to individual strategies to a second. Interestingly, four items (two outcomes and two strategies) - "to be completely free from pain at rest", "to be completely free from pain on movement", "using epidural analgesia for 48 hours" and "optimizing fluid balance" - loaded onto a third factor, suggesting additional structure not appreciated in the original questionnaire design (Figure 11.1).

Table 11.1 Patient demographics and clinical details

\begin{tabular}{|c|c|c|c|c|}
\hline & Troms $\varnothing(n=36)$ & Edinburgh $(n=38)$ & Maastricht $(n=35)$ & Total $(n=109)$ \\
\hline \multicolumn{5}{|l|}{ Operation n(\%) } \\
\hline Hepatic resection & $2(6)$ & 38 (100) & $20(57)$ & $60(55)$ \\
\hline Colorectal/small bowel & $19(53)$ & - & $15(43)$ & $34(31)$ \\
\hline Esophagogastric & $8(22)$ & - & - & $8(7)$ \\
\hline Pancreatic & $2(6)$ & - & - & $2(2)$ \\
\hline Other & $5(13)$ & - & - & $5(4)$ \\
\hline \multicolumn{5}{|l|}{ Length of Stay } \\
\hline Days, median (range) & $5(3-96)$ & $7(4-38)$ & $7(3-30)$ & $7(3-96)$ \\
\hline \multicolumn{5}{|l|}{ Complications $\mathrm{n}(\%)$} \\
\hline Yes & $11(31)$ & $17(45)$ & $14(40)$ & $42(39)$ \\
\hline No & $25(69)$ & $21(55)$ & $21(60)$ & $67(61)$ \\
\hline \multicolumn{5}{|l|}{ ICU admission n(\%) } \\
\hline Yes & $6(17)$ & $2(5)$ & $2(6)$ & $10(8)$ \\
\hline No & $30(83)$ & $36(95)$ & $33(94)$ & $99(92)$ \\
\hline \multicolumn{5}{|l|}{ Readmission n(\%) } \\
\hline Yes & 7 (19) & $4(11)$ & $6(17)$ & $17(16)$ \\
\hline No & $29(81)$ & $34(89)$ & $29(83)$ & $92(84)$ \\
\hline \multicolumn{5}{|l|}{ Pathology n(\%) } \\
\hline CLM & $2(6)$ & $25(66)$ & $19(54)$ & $46(42)$ \\
\hline Cholangiocarcinoma & - & $2(5)$ & - & $2(2)$ \\
\hline $\mathrm{HCC}$ & - & $5(13)$ & - & $5(4)$ \\
\hline Other & $4(11)$ & $3(8)$ & - & $7(6)$ \\
\hline Primary adenocarcinoma & $28(77)$ & - & $16(46)$ & $44(40)$ \\
\hline Benign & $2(6)$ & $3(8)$ & - & $5(5)$ \\
\hline \multicolumn{5}{|l|}{ Previous hospital stay n(\%) } \\
\hline Yes & $27(75)$ & $32(84)$ & $32(91)$ & $91(83)$ \\
\hline No & $9(25)$ & $6(16)$ & $3(9)$ & $18(17)$ \\
\hline \multicolumn{5}{|l|}{ Age (years) } \\
\hline Median (range) & $66(35-93)$ & $63.5(32-79)$ & $65(36-85)$ & $64(35-93)$ \\
\hline \multicolumn{5}{|l|}{$\operatorname{Sex} n(\%)$} \\
\hline $\mathrm{M}$ & $25(69)$ & $25(66)$ & $20(57)$ & $70(64)$ \\
\hline $\mathrm{F}$ & $11(31)$ & $13(34)$ & $15(43)$ & $39(36)$ \\
\hline \multicolumn{5}{|l|}{ ASA n(\%) } \\
\hline 1 & $2(6)$ & $4(11)$ & $5(14)$ & $11(10)$ \\
\hline II & $25(69)$ & $28(74)$ & $17(49)$ & $70(64)$ \\
\hline III & $8(22)$ & 6 (15) & $13(37)$ & $27(25)$ \\
\hline IV & $1(3)$ & - & - & $1(1)$ \\
\hline \multicolumn{5}{|l|}{ Employment status n(\%) } \\
\hline Employed & $12(33)$ & $16(42)$ & $7(20)$ & $35(32)$ \\
\hline Retired & $22(61)$ & $18(47)$ & $22(63)$ & $62(57)$ \\
\hline Unemployed & $2(6)$ & $4(11)$ & $6(17)$ & $12(11)$ \\
\hline
\end{tabular}


To be independently mobile in hospital as soon as possible To be able to return to all daily activities as soon as possible

To be able to move the bowels as soon as possible To be completely free of nausea - not feeling or being sick

To be able to eat and drink as soon as possible

To be discharged from hospital as soon as possible

Antimicrobial prophylaxis and skin preparation

Promoting early and scheduled mobilisation

Stimulation of gut motility

Allowing normal diet at will after the operation

Avoiding nasogastric tube after the operation

Avoiding pre-anaesthetic sedative medication

Oral carbohydrate loading preoperatively

Avoiding a wound drain

Preoperative fasting kept at absolute minimum

Avoiding oral bowel preparation preoperatively

Preoperative counselling by nurse, anaesthetist and surgeon

Optimizing Fluid balance

To be completely free of pain at rest

To be completely free of pain upon movement

Using epidural analgesia for approximately

48 hrs postop

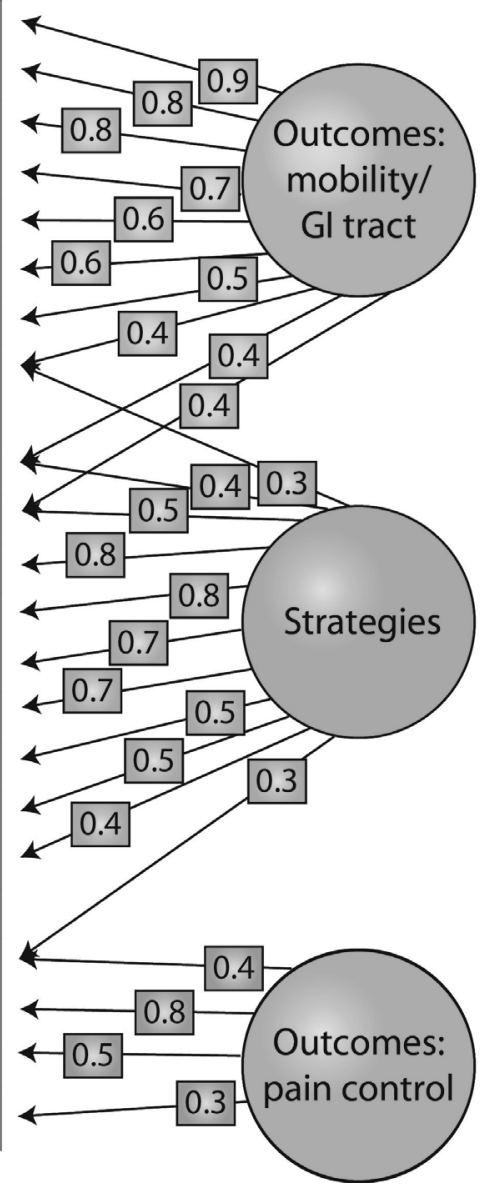

Figure 11.1 Factor analysis of questionnaire items. Each number represents the factor loading which can be thought of as a correlation coefficient between a questionnaire item and one of the three factors. Three distinct groups were identified as illustrated above. This signifies uniformity of scoring patterns occurring within these three groups.

\section{Patient outcomes}

The overall scores were high with the lowest median score being 8/10. The preoperative impressions of patients awaiting surgery were unchanged by the subsequent surgery, with no significant differences observed between pre- and postoperative scores (i.e. $P>0.05$ for each comparison following adjustment for multiple comparisons), as determined by the Wilcoxon signed-rank tests (Figure 11.2). 
Patients scored freedom from nausea (median 10 IQR 8-10) and freedom from pain at rest (median 10 IQR 8-10) as the two most important factors. Achieving independent mobility was also scored highly by patients (median 9, IQR 8-10). Early discharge (median 9, IQR 6.5-9) and early return of bowel movements (median 9, IQR 7.25-10) received the lowest scores when taking the IQR into account (Figure 11.2).

\section{Patient strategy}

Patients highlighted pre-operative counseling (median 10, IQR 8.75-10) and avoiding infection in hospital (median 10, IQR 9-10) as a priority in achieving recovery (Figure 11.2).

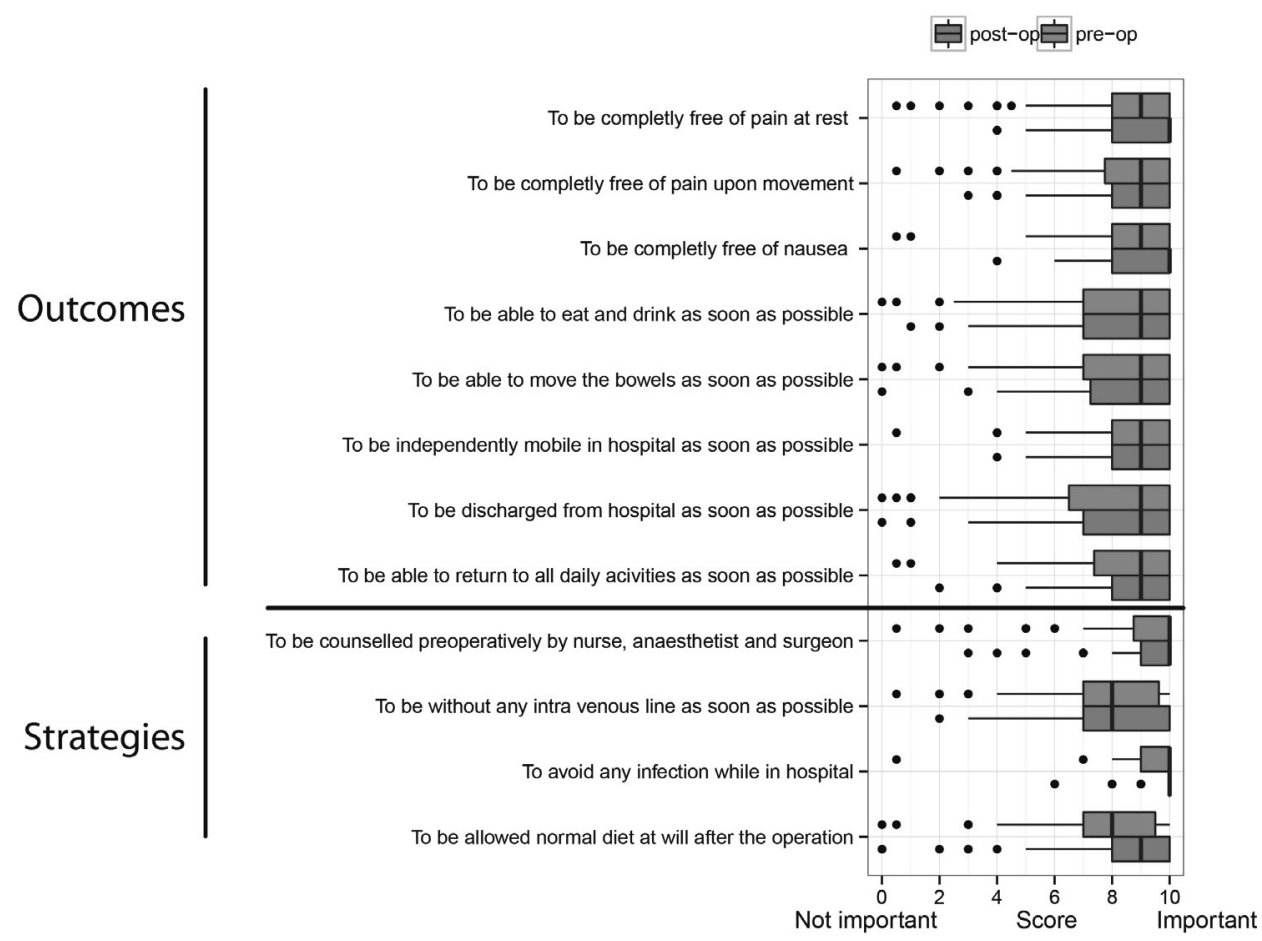

Figure 11.2 Patient ratings of ERAS outcomes and strategies. Pre-operative scores are compared with postoperative scores for each item. Wilcoxon signed-rank test was used to compare scores. No statistically significant differences were observed between scores.

\section{Care provider outcomes}

Outcomes were in general scored highly by the care providers with all items being scored with a median of $7 / 10$ or higher. The outcomes scored as most important by 
care providers were control of nausea (median 9, IQR 8-10) and being free from pain at rest (median 9, IQR 8-10) (Figure 11.3).

Being able to move the bowels as soon as possible was scored the lowest (median 7, IQR $5-8$ ) by the care providers. Also being discharged from hospital as soon as possible received lower scores (median 8, IQR 6-9) as did returning to daily activities as soon as possible (median 8, IQR 5.5-10) (Figure 11.3).

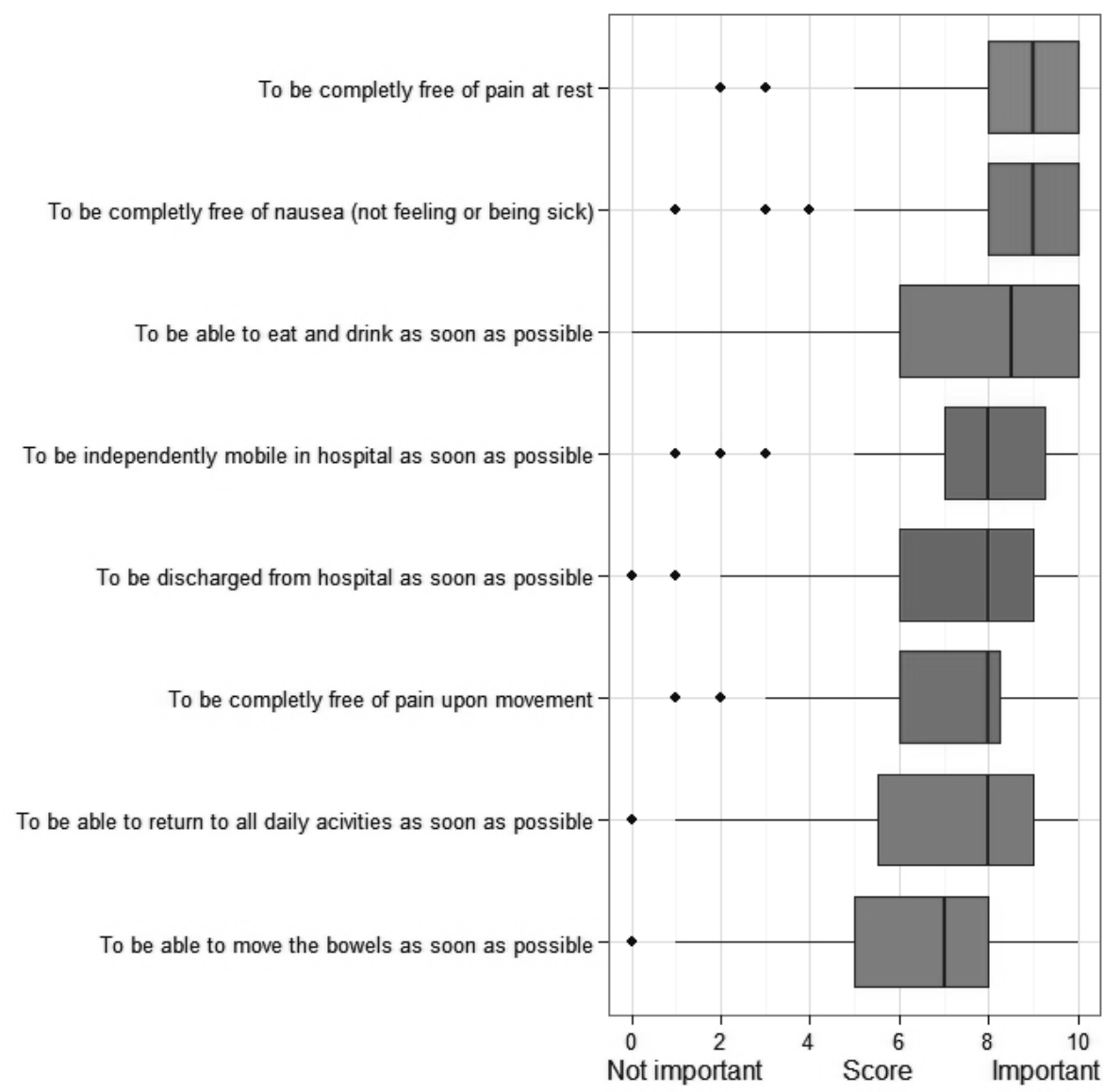

Figure 11.3 Combined care providers ratings of ERAS outcomes.

\section{Care provider strategy}

The range of scores was more varied in this area of responses. The highest rated items of care strategy as scored by the care providers were: provision of pre-operative 
counseling (median 10, IQR 9-10); promotion of early mobilization (median 9.5, IQR 910) and optimization of IV fluid administration (median 9, IQR 8-10) (Figure 11.4).

Avoiding wound drains (median 7, IQR 4.5-8), avoiding bowel preparation (median 7, IQR 4-8) and avoiding anesthetic pre-medication (median 6.5, IQR 3.5-8) were scored the lowest (Figure 11.4).

Sub-group analyses using the Kruskal Wallis test were performed to compare scores of each item by individual specialties of care providers and between the care provider nationalities. Following adjustment for multiple comparisons no significant differences between care provider specialty nor nationality scores were observed.

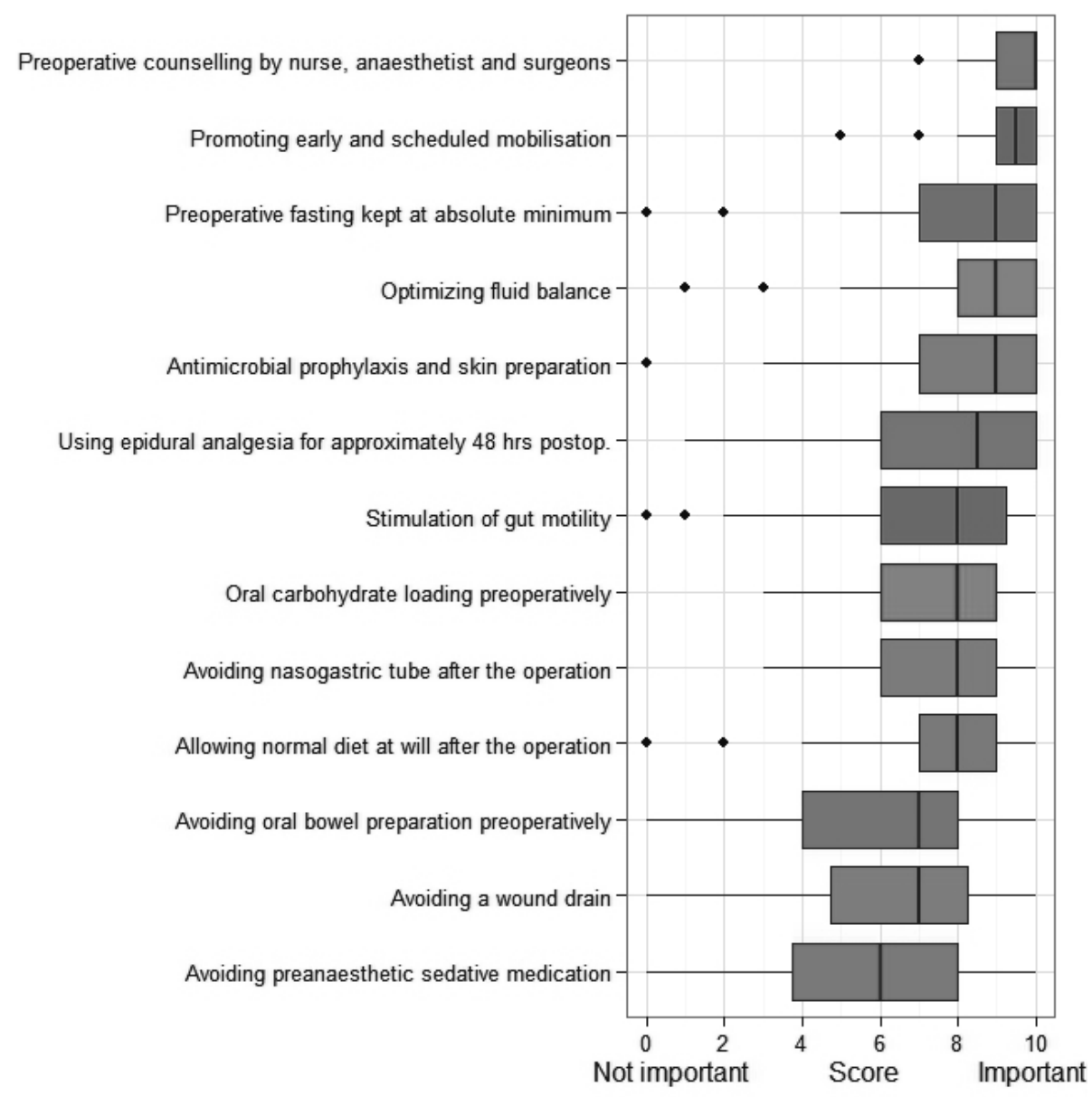

Figure 11.4 Combined care providers' ratings of ERAS strategies. 


\section{DISCUSSION}

This study investigated the opinions and perceptions of both providers and receivers of enhanced recovery protocols following major abdominal surgery in three European countries. It is the first study of its kind.

The novel questionnaire was designed for the purposes of this study and validated successfully. Good internal consistency was observed and the factor analysis performed revealed a logical separation of items into ERAS outcomes and strategies. This suggests consistent and distinct scoring patterns within these groups of questionnaire items indicating satisfactory validity of the items selected. These results also reflect the consistently high scores throughout the survey and overall support for the outcomes and strategies used in the questionnaire.

The post-operative response rate or eighty-one per cent was satisfactory. The nonresponders underwent a greater proportion of esophagogastric resections and fewer hepatic resections than those who completed the post-operative questionnaire. Although the ICU admission rate was higher in this sub-group, readmission and complication rates remained unchanged. This likely reflects a tendency for esophagogastric resections to be managed initially in ICU routinely, which was not the case for hepatic resections, and their absence was not thought to adversely influence the post-operative responses observed.

Patients attributed high importance to all of the ERAS aims and strategies asked of them. This represents concordance between patients' beliefs and current recommendations by ERAS experts ${ }^{8}$ and this finding should be used to encourage and develop ERAS implementation.

Previous studies have also shown that compliance with ERAS protocols can be compromised resulting in deviation from care plans ${ }^{5}$. However, our results show that patient support for the ERAS process is high and therefore we suggest it is unlikely that lack of patient acceptance is the main cause of deviation from protocols. Moreover, we did not show a difference in patient opinion pre- and post-operatively, suggesting that patient support for such care plans does not change even after having gained experience of the care principles.

A qualitative assessment of patient experience following ERAS surgery ${ }^{9}$ found that decreased satisfaction was reported in a subgroup of patients who suffered a complication following discharge. Our survey sample included patients who suffered complications at rates that would be expected from these types of surgery. This did not cause significantly different pre-operative and post-operative patient scoring and so we do not concur with the view that the development of a complication negatively impacts on patients' support for the ERAS principles.

The clinicians also scored highly for the majority of targets and strategies and good inter-specialty agreement was observed, representing streamlined support for ERAS principles. Opinions of different professional groups involved in delivering ERAS 
programs has not been assessed before and represents a novel finding of the study and justification for continued implementation.

However, although our scores were high there were some areas of relatively lower scoring. There was a broad range of scores for the importance of epidurals, particularly from surgeons (range 1-10). This reflects the controversy that surrounds routine epidural use ${ }^{15}$. The use of alternative analgesic modalities, such as intrathecal analgesia and continuous wound infiltration, has become increasingly popular ${ }^{10,11}$. The UK Department of Health's Enhanced Recovery Partnership Program (ERPP) consensus opinion $^{12}$ has subsequently advocated less routine epidural use and increased alternative analgesic methods.

Fluid balance optimization was scored highly by care providers. Both inappropriate fluid restriction and excessive fluid administration have been shown to be associated with prolonged hospital stays ${ }^{13}$ and increased post-operative morbidity ${ }^{14}$ reflecting the importance of accurate fluid balance. Epidural use is associated with vasodilation and hypotension and can be associated with excessive intravenous fluid administration ${ }^{15}$. It may be that these perceived drawbacks associated with epidural use are contributory to its lower rating. Indeed the factor analysis identified epidural use, fluid balance and minimization of pain as a distinct group separate from ERAS outcomes and strategies, reflecting an independent but uniform scoring pattern for these items by the care providers.

Strategies scored lowest overall by care providers were the avoidance of post-operative abdominal drains, oral bowel preparation and pre-anesthetic sedatives. There is highlevel evidence in colorectal surgery advocating against drain insertion ${ }^{16}$. Similarly, there is good evidence suggesting no benefit of routine bowel preparation prior to abdominal surgery ${ }^{17}$.

The stimulation of gut motility was also scored in the lower half of results, particularly by anesthetists and, to a lesser degree, surgeons. Liberal laxative use to prevent constipation and achieve restoration of bowel function is often advocated as a preventative measure against post-operative ileus ${ }^{18}$ and is a component of many enhanced recovery protocols. However the care provider responses were at odds with this evidence.

These results may be partly due to of the presence of resections other than colorectal in the study population where there is less clear evidence for the benefits of drain avoidance and less relevance of bowel preparation and stimulation. However, the reluctance to abandon previously well-established practice despite evidence to the contrary is recognized in the literature ${ }^{6,19}$. This is a potential obstacle to the implementation and continued establishment of ERAS principles and is a phenomenon that continues to be observed ${ }^{20}$ and requires attention when addressing failing ERAS implementation.

Potential drawbacks to the study are the relatively small sample size and the postoperative response rate. It is also acknowledged that due to the small sample size and 
the high concordance in the overall scoring it is not possible to determine which items are considered more important relative to each other. However it is possible to determine those items scored highest and lowest and an appropriate qualitative assessment of these items was performed. Additionally, the overall high internal consistency of the results suggests that the sample provides valid results and is an acceptable number for this descriptive analysis.

The survey was performed on patients, for the most part, undergoing major open abdominal surgery for malignant disease processes. This therefore represents a population representative of the majority of ERAS recipients however is not strictly relevant to non-abdominal surgery.

In conclusion, this novel study has shown good patient and multidisciplinary care provider approval for the principles of enhanced recovery care after surgery and supports on going development and implementation of such programs. It highlights potential areas of concern for care providers, namely epidural use and fluid administration. These issues remain controversial and could be a focus for future research. Lastly, we identified several items scored low priority by care providers where there is a strong evidence base to the contrary. This highlights a potential barrier to ERAS implementation and is an area that requires consideration and education if continued sustainability and development of ERAS programs is to be achieved. 


\section{REFERENCES}

1. Adamina M, Kehlet H, Tomlinson GA, Senagore AJ, Delaney CP. Enhanced recovery pathways optimize health outcomes and resource utilization: a meta-analysis of randomized controlled trials in colorectal surgery. Surgery 2011; 149: 830-840.

2. Coolsen MM, Wong-Lun-Hing EM, van Dam RM, van der Wilt AA, Slim K, Lassen K, Dejong CH. A systematic review of outcomes in patients undergoing liver surgery in an enhanced recovery after surgery pathways. HPB (Oxford) 2013;15:245-251.

3. Tatsuishi W, Kohri T, Kodera K, Asano R, Kataoka G, Kubota S, Nakano K. Usefulness of an enhanced recovery after surgery protocol for perioperative management following open repair of an abdominal aortic aneurysm. Surg Today 2012;42:1195-200.

4. Savaridas T, Serrano-Pedraza I, Khan SK, Martin K, Malviya A, Reed MR. Reduced medium-term mortality following primary total hip and knee arthroplasty with an enhanced recovery program. Acta Orthop 2013;84:40-43.

5. Smart N, White P, Allison A, Ockrim J, Kennedy R, Francis N. deviation and failure of enhanced recovery after surgery following laparoscopic colorectal surgery: early prediction model. Col Dis 2012;14: e727-e734

6. Lassen K, Hannemann P, Ljungqvist O, Fearon K, Dejong CH, von Meyenfeldt MF, Hausel J, Nygren J, Andersen J, Revhaug A; Enhanced Recovery After Surgery Group. Patterns in current perioperative practice: survey of colorectal surgeons in five northern European countries. BMJ 2005;330:1420-1421.

7. Grol R, Grimshaw J. From best evidence to best practice: effective implementation of change in patients' care. Lancet 2003;362:1225-30.

8. Fearon KC, Ljungqvist $\mathrm{O}$, Von Meyenfeldt $\mathrm{M}$, Revhaug A, Djong $\mathrm{CH}$, Lassen $\mathrm{K}$. Enhanced recovery after surgery: a consensus review of clinical care for patients undergoing colonic resection. Clin Nutr 2005;24:466-477.

9. Blazeby JM, Soulsby M, Winstone K, King PM, Bulley S, Kennedy RH. A qualitative evaluation of patients' experiences of an enhanced recovery programme for colorectal cancer. Colorectal Dis 2010;12: e236-e242.

10. Koea JB, Young Y, Gunn K. Fast track liver resection: the effect of a comprehensive care package and analgesia with single dose intrathecal morphine with gabapentin or continuous epidural analgesia. HPB Surg 2009;2009:271986.

11. Revie EJ, McKeown DW, Wilson JA, Garden OJ, Wigmore SJ. Randomized clinical trial of local infiltration plus patient-controlled opiate analgesia vs. epidural analgesia following liver resection surgery. $H P B$ (Oxford) 2012;14:611-8.

12. Knott A, Pathak S, McGrath JS, Kennedy R, Horgan A, Mythen M, Carter F, Francis NK. Consensus views on implementation and measurement of enhanced recovery after surgery in England: Delphi study. BMJ Open 2012;12;2(6). pii: e001878.

13. Tambyraja AL, Sengupta F, MacGregor AB, Bartolo DCC, Fearon KCH. Patterns and clinical outcomes associated with routine intravenous sodium and fluid administration after colorectal resection. World J Surg 2004;28:1046-1051,

14. Wakeling HG, McFall MR, Jenkins CS, Woods WG, Miles WF, Barclay GR, Fleming SC. Intraoperative oesophageal Doppler guided fluid managementshortens postoperative hospital stay after major bowel surgery. Br J Anaesth 2005;95: 634-642.

15. Low J, Johnston N, Morris C. Epidural analgesia: first do no harm. Anaesthesia 2008;63:1-3.

16. Petrowsky H, Demartines N, Rousson V, Clavien PA. Evidence-based value of prophylactic drainage in gastrointestinal surgery: a systematic review and meta-analyses. Ann Surg 2004;240:1074-1084.

17. Wille-Jørgensen P, Guenaga KF, Matos D, Castro AA. Pre-operative mechanical bowel cleansing or not? an updated meta-analysis. Colorectal Dis 2005;7:304-310.

18. Kehlet H. Postoperative ileus - an update on preventive techniques. Nat Clin Pract Gastroenterol Hepatol 2008; 5:552-558.

19. Maessen J, Dejong CH, Hausel J, Nygren J, Lassen K, Andersen J, Kessels AG, Revhaug A, Kehlet H, Ljungqvist $\mathrm{O}$, Fearon KC, von Meyenfeldt MF. A protocol is not enough to implement an enhanced recovery programme for colorectal resection. Br J Surg 2007;94:224-231. 
20. Kahokehr A, Robertson P, Sammour T, Soop M, Hill AG. Perioperative care: a survey of New Zealand and Australian colorectal surgeons. Colorectal Dis 2011;13:1308-1313. 


\section{CHAPTER 12}

General discussion and summary 
Chapter 12 


\section{GENERAL DISCUSSION AND SUMMARY}

The principal aim of this thesis was to investigate the feasibility of ERAS programs for HPB surgery. There is abundant evidence demonstrating that ERAS programs are preferred over traditional care in colonic surgery. Also in other surgical fields ERAS programs have been proven to result in shorter hospital stay, lower morbidity rates and reduced hospital costs ${ }^{1-4}$. However, until now it remained unclear whether it is also feasible to implement ERAS programs in major abdominal surgery such as HPB surgery. In summary, the aims of the present thesis were as follows:

1) To critically appraise the literature regarding ERAS programs for HPB surgery and endpoints in pancreatic surgery (Part I, chapter $2,3,4,5$ )

2) To develop perioperative ERAS guidelines for pancreaticoduodenectomy (Part II, chapter 6)

3) To initiate a trial evaluating the effect of prophylactic intra-abdominal drainage for patients undergoing pancreaticoduodenectomy in an ERAS program (Part II, chapter 7)

4) To evaluate the implementation of an ERAS program in HPB surgery (Part III, chapter $8,9,10,11$ )

\section{Part I: Critical appraisal of literature: ERAS programs in HPB surgery and} endpoints in pancreatic surgery

The first aim of this thesis was to assess what is already known about ERAS programs in HPB surgery and compare existing studies. We performed a systematic review of ERAS programs in pancreatic surgery (chapter 2). Also, a meta-analysis was performed but only on articles related to patients undergoing pancreaticoduodenectomy (PD) because this is the most common surgical pancreatic procedure and is considerably different from other forms of pancreatic surgery. From the eight studies described in this review we concluded that an ERAS protocol helps to shorten (hospital) length of stay (LOS) without compromising morbidity and mortality. Meta-analysis of four studies focusing on PD showed that there were no differences in readmission or mortality rates and that morbidity rates were significantly lower for patients managed according to ERAS principles. We also performed a systematic review of ERAS programs for liver surgery (chapter 3). Six studies were included in this review and analysis showed that an ERAS program can be successfully implemented in liver surgery without altering morbidity, mortality or readmission rates. For both reviews, some limitations are mentioned. First, there were no randomized controlled trails (RCTs) available for inclusion. Only a few randomized trials comparing ERAS with traditional care in colorectal surgery exist ${ }^{2,3}$ but no such trials have been conducted in the field of liver and pancreatic surgery. This is probably due to the fact that randomized controlled trials are difficult to organize for multimodal recovery programs under greatly varying conditions. Moreover, several protocol elements of an ERAS program have already become standard practice in many 
hospitals during the last decade (e.g., thromboprophylaxis, prevention of hypothermia with Bair Huggers, early mobilization). This raises ethical concerns with respect to randomization because patients in the control group may not receive specific evidencebased interventions.

Second, the methodological quality of the studies was variable since they consisted mostly of retrospective or prospective case series and comparative studies based on historical controls. Furthermore, individual studies used slightly different study protocols. The items incorporated in the various protocols were not identical making it difficult to compare the studies. Also, the degree of compliance to the ERAS-protocol was not reported in the studies. Finally, definitions of complications were ill-defined in the studies and complications were not registered in a standardized classification system (e.g. the Dindo-Clavien classification). We concluded that there is need for a standardized and procedure-specific ERAS program for pancreatic and liver surgery to be implemented and used in future studies. Furthermore, standardized registration systems should be used to classify complications and uniform definitions for specific complications must be maintained. It is also important to describe adherence to protocol in studies reporting on ERAS programs.

The omission of prophylactic postoperative intra-abdominal drains is one of the components of an ERAS program in colonic surgery. For most abdominal surgical procedures there is little evidence indicating the benefits of drains. It certain cases they may even be considered harmful ${ }^{5-7}$. However, in pancreatic surgery surgeons are reluctant to omit prophylactic intra-abdominal drains because they can serve to indicate a bleeding or leakage of the pancreatic anastomosis. We performed a systematic review and cumulative meta-analysis of three studies comparing patients undergoing PD in a drain and no-drain group (chapter 4). In this study, a traditional meta-analysis of the available evidence pointed to a significantly lower complication rate in the group without routine drainage. However, when a cumulative meta-analysis was performed with adjustment for repeated testing and heterogeneity, this difference was no longer statistically significant. In conclusion, the routine use of abdominal drains after pancreatic resection may carry a higher risk of complications but the evidence remains inconclusive. A recently published review on the use of abdominal drains after pancreatic resections adopted a slightly different approach, using a standard MantelHaenszel random-effects model for meta-analysis and separate evaluations of overall and specific complications, respectively ${ }^{8}$. In line with our findings, this review concluded that more evidence is required before any firm conclusions on the use of prophylactic abdominal drains can be drawn. Evaluation of early (postoperative day 3 ) versus late (postoperative day 5 and beyond) drain removal has been examined in a RCT of Bassi et al. ${ }^{9}$. Early removal of the drain in patients at low risk of pancreatic fistula (amylase value in drains $<5,000 \mathrm{U} / \mathrm{I}$ at postoperative day 3) was associated with a significantly decreased rate of pancreatic fistula, abdominal and pulmonary complications. Another prospective study comparing drain removal on day 4 versus day 
8 showed that drain removal on postoperative day 4 was an independent factor in reducing the incidence of complications after PD including intra-abdominal infections ${ }^{10}$. So, until further data are available a conservative approach with systematic postoperative drainage and early removal in patients at low risk of pancreatic fistula (firm pancreas, wide pancreatic duct) is recommended. However, given the inconclusive evidence for drainage after PD, further exploration by RCTs is needed and differences in postoperative outcomes between early drain removal and a no-drain regimen after PD should be examined.

Few RCTs have been performed in pancreatic surgery ${ }^{10,11}$. An important factor contributing to this is the relatively low incidence of pancreatic disease. Furthermore, in RCTs focusing on pancreatic surgery the usefulness of outcomes as surgery-related mortality or specific PD-related morbidity as a primary endpoint is limited because individually they have a relatively low incidence. Consequently, large sample sizes are needed to obtain adequate statistical power. Another problem in research into pancreatic surgery is that comparability of studies is low due to ill-defined definition of complications and a lack of standardized complication registration systems. Use of a PD-specific composite endpoint containing outcome parameters that are well-defined and of significant severity could overcome this problem. In chapter $\mathbf{5}$ the development of a PD-specific composite endpoint (CEP) is described. In a CEP several outcome parameters are combined to form a single dichotomous endpoint ${ }^{12}$. The individual parameters that are combined in a CEP should also be dichotomous, highly specific for the given surgical procedure and of clinical relevance. Scoring a CEP results in a higher incidence than the incidence of individual specific endpoints. Using CEP as a primary endpoint therefore increases statistical power and allows for smaller sample sizes when performing RCTs. Because CEPs are well-defined they enhance the performance of RCTs and subsequent meta-analysis as well as their quality and comparability. The PDspecific CEP components were selected after a systematic review of the literature and consensus between 25 international pancreatic surgeons by means of a web-based survey. Consensus on definitions for these components was also reached. From a total of eighteen single component endpoints, intra-abdominal abscess, sepsis, post- PDhemorrhage, bile leakage, gastrojejunostomy leakage, leakage of the pancreatic anastomosis, delayed gastric emptying and operative mortality within 90 days were selected to be included in the PD-specific CEP. Since it is imperative for the components to have a similar clinical relevance or severity ${ }^{13}$, all individual components of the CEP have to be classified with a Dindo-Clavien grade of 3 or more ${ }^{14}$. The event rate and effect of the PD-specific CEP was consequently evaluated in two prospective cohorts of patients who underwent PD in two high-volume HPB centers (MUMC+, Maastricht and Royal Free Hospital, London). The incidence of the individual components with a DindoClavien grade $\geq 3$ varied between $0.5 \%$ and $15.7 \%$ in the MUMC+ and from $1.7-8.3 \%$ in the Royal Free Hospital. Subsequently, the incidence of the PD-specific CEP was calculated and was found to be $24.7 \%$ in the Maastricht cohort and $23.3 \%$ in the 
London cohort. Compared to the use of single-component primary endpoints, the incidence rates obtained with a PD-specific CEP lead to a two-fold reduction of the theoretical, calculated sample size for an adequately powered RCT. A drawback of using CEPs is that its individual components may be ill-defined and show differences in severity or incidence. Furthermore, the components may not be affected in the same manner by the intervention ${ }^{15}$. In developing our CEP we tried to address these issues in a systematic way by reaching international consensus on definitions of the individual components and by classifying only complications with a Dindo-Clavien grade of 3 or more. Quality of life was not included in this CEP since experts did not consider this to be PD-specific enough. Even so, patient perception should not be disregarded since patients may consider the consequences of complications (e.g. pain, fatigue or confusion) as more critical than physicians do. This issue should be addressed in future research on CEPs.

It may be also be necessary to develop CEPs for other forms of pancreatic surgery as well as for drug-related pancreatic research. Furthermore, weighing of specific components of a CEP, not only by clinicians but also by patients, might be considered in future work. The use of CEPs in RCTs for surgical research seems promising but prospective evaluation is needed.

\section{Part II: Development of ERAS guidelines and a multicenter trial for pancreaticoduodenectomy}

As pointed out in chapter 2, studies on the implementation of ERAS programs in pancreatic surgery and in particular in PD employ ERAS protocols with a varying set of elements $^{16-18}$ or report no protocol at all ${ }^{19}$. These studies are therefore not fully comparable. Chapter 6 describes the development of a consensus framework of ERAS guidelines for PD by the international ERAS group. This framework has come about after a systematic literature search and grading of the selected studies. The strength of evidence and conclusive recommendations for each protocol element ( 27 in total) were assessed and agreed by all authors in a meeting in May 2012 and taken together to form ERAS guidelines for PD.

ERAS programs have been strongly associated with reduced length of hospital stay (LOS) but this may not be the best indicator of the quality of functional recovery, which an ERAS program pursues ${ }^{20}$. Emphasis must also be placed on reducing morbidity. Multimodal ERAS programs are complex interventions that pose significant challenges to evaluation by conventional RCTs ${ }^{21}$. The most obvious of these challenges are standardization of the intervention and ethical and practical concerns. This may, to some extent, explain the relative lack of RCTs evaluating ERAS programs and the somewhat limited effect that has been shown on endpoints other than LOS. It may be reasonable to propose that, if RCTs have proven the benefit, a protocol of items, which are supported by RCTs, should not need analysis in an RCT itself. Feasibility, however, must be ensured. Hence, multicenter and multinational prospective validation of this 
perioperative care protocol for PD in consecutive cohorts of patients undergoing PD is warranted.

In the ERAS guidelines for PD no consensus was reached regarding omission or inclusion of prophylactic intra-abdominal drainage since the evidence from literature remained inconclusive, as also shown in chapter 4. For this reason, we decided to design a multicenter RCT comparing prophylactic abdominal drainage with a no-drain policy in patients undergoing pancreaticoduodenectomy (chapter 7). This trial will be carried out in 10 different hospitals across the Netherlands and Europe. All patients in this study are treated according to the developed ERAS guidelines as posed in chapter $\mathbf{6}$. Also, the recently developed composite endpoint for pancreaticoduodenectomy will be used as one of the outcomes as validation (chapter 5). A sample size of 360 patients was calculated on the primary endpoint (need for radiological or surgical interventions). The trial started enrolling patients from January 2013 in MUMC+. The other hospitals followed in the months thereafter after approval of the Ethical Committee. With this multicenter international RCT we hope to shed light on the risk and benefits of omitting a postoperative intra-abdominal drain after pancreaticoduodenectomy and gain conclusive evidence whether a no-drain policy is better than early removal of the perianastomotic drain.

\section{Part III: Evaluation of implementing an ERAS program in liver and pancreatic surgery}

In the Maastricht University Medical Center (MUMC), the ERAS program for colonic surgery was implemented from 2000 onwards $^{22}$. In 2005, a pilot was started in Maastricht and Edinburgh resulting in the successful implementation of the ERAS program in liver surgery (chapter 8). This was a prospective study involving 61 patients. Outcomes were compared with those in a consecutive series of 100 patients who underwent liver resection before the start of the study. Fifty-six patients (92\%) in the ERAS group tolerated fluids within $4 \mathrm{~h}$ of surgery and a normal diet on day 1 after surgery. Median hospital stay, including readmissions, was 6 days compared with 8 days in the control group $(P<0.001)$. There were no significant differences in rates of readmission ( $13 \%$ and $10.0 \%$ respectively), morbidity $(41 \%$ and $31 \%)$ and mortality $(0 \%$ and $2.0 \%$ ) between ERAS and control groups. Nasogastric tubes (NGT) were removed on the day of surgery in $92 \%$ of the patients in the ERAS group, whereas in the control group none of the NGTs was removed on the day of surgery $(P<0.001)$. Also, prophylactic abdominal drains were used only in $2 \%$ in the ERAS group versus $66 \%$ in the control group $(P<0.001)$. Fifty-two $(85 \%)$ of 61 patients in the ERAS group were completely mobile on the third day after operation and 29 (48\%) were discharged from the hospital within 5 days. We concluded that an ERAS program can be safely and effectively implemented in liver surgery. In this study, the ERAS program allowed for early oral intake, promoted postoperative recovery and a reduced hospital stay. The impact of the ERAS liver program became more evident at the end of the 2006 pilot. 
Several ERAS items were also implemented in pancreatic surgery but a standardized protocol was not yet available. In 2009 a concept protocol was established for PD and used ever since. The ERAS guidelines for PD (chapter 6) were implemented from 2013 onwards and will be evaluated in a multicenter international prospective project.

Chapter 9 describes an examination of patient characteristics, indications and outcome after PD in the period between January 1995 and January 2012 in MUMC and also evaluates the effects of implementing an ERAS program for PD. This time period is divided in three groups according to patients receiving no or (partial) ERAS perioperative care: Group 1 (1995-2005) "no ERAS", group 2 (2006-2008) "ERAS-like" and group 3 (2009-2011) "ERAS". Median length of stay (LOS) was significantly reduced from 20 days in group 1 to 13 days in group 2 and 14 days in group $3(P=0.001)$. Patients discharged without complications had a median LOS of 15, 10 and 9 days, respectively, in group 1, 2 and $3(P<0.001)$. Over time, resection volume increased significantly in the authors' unit with more pylorus-preserving pancreaticoduodenectomies (PPPDs) being performed than classical Whipple's procedures. Complication rates as well as mortality and readmission rates did not change. We concluded that an ERAS program for PD is feasible and that the implementation and increasing resection volume probably both contributed to a decrease of LOS without compromising other outcomes.

In addition, Chapter $\mathbf{1 0}$ demonstrates that it is also feasible to subject patients of advanced age to an ERAS program. We prospectively evaluated a group of 110 patients undergoing PD and divided them in two groups (below 65 years and above 70 years of age). We found that compliance with the different aspects of the protocol, number of readmissions, morbidity and mortality rates were not significantly different between both groups, concluding an enhanced recovery program is also feasible for patients above 70 years of age undergoing a PD.

Since the ERAS colon protocol was already implemented in our clinic from 2000 onwards some major changes in different areas (anesthesiology, on the ward and physiotherapy department) had already been instituted, which made it easier to implement ERAS protocols for liver and pancreatic surgery as well. However, this does not hold true for all protocol elements. For instance, owing to tradition in the anesthesiology department NGTs were still used extensively in liver and pancreatic surgery and removing NGTs directly after surgery meant a major change in clinical practice for these kinds of operations. As shown in chapter 8 and 9 this change in routine did not result in more pulmonary complications. Also, especially in PD, postoperative enteral nutrition starting with fluid intake on the day of surgery and solid intake from day 1 was an evident change of practice, which was also tolerated well by most of the patients. For the ERAS PD protocol some elements of the protocol (e.g. the early removal of the urine catheter and abdominal drain) were not sufficiently complied with. The fact that implementing evidence-based care in practice can be challenging is shown by several studies. Lassen et al. performed a survey in 200 hospitals in five European countries distributed via departments of surgeons participating in the ERAS 
group for colonic surgery. It appeared that perioperative routines in colonic cancer surgery differed widely between the countries and deviated considerably from the recommendations of the ERAS protocol (e.g. bowel preparation was still applied in the majority of the clinics as well as the use of NGTs ${ }^{23}$. Maessen et al. performed an international study to evaluate the implementation of an enhanced recovery program including 425 patients undergoing colonic resection ${ }^{24}$. This study showed that protocol compliance was high before and during the surgical procedure but was low in the immediate postoperative phase (ranging from $36-85 \%$ for various protocol items). Furthermore, only $31 \%$ of the patients were discharged on the day of functional recovery. Most of the patients were discharged on median postoperative day (POD) 5 while functional recovery was already reached on POD 3 . These results are in line with results for the ERAS program for PD as described in chapter 10 and suggest more effort should be put into improving compliance in the immediate postoperative phase, which could further accelerate recovery (e.g. continuous education of nurses and staff and the use of self-reporting diaries). Moreover, postoperative care should be planned better and re-organized to really discharge patients on days they meet the functional recovery criteria. Similarly, in a present study about fast track laparoscopic liver surgery in our unit functional recovery was reached two days before the actual discharge $\mathrm{e}^{25}$. This poses the question whether LOS is actually an appropriate readout to evaluate the impact of an ERAS program rather than the day functional recovery or discharge criteria are met. Questions are raised about some perioperative care elements of an ERAS protocol being more important than others. In a recent paper a multivariable analysis of care elements influencing LOS in 2,485 patients undergoing colonic surgery showed that cessation of IV fluids, mobilization on postoperative day 1, and postoperative administrations of laxatives (magnesium oxide) were associated with shortened LOS $^{26}$. Hence, ERAS programs may accelerate restoration of gut motility. In PD, this might help to lower the incidence of delayed gastric emptying (DGE). A lower incidence of DGE has been demonstrated before in patients undergoing PD in an enhanced recovery pathway, although this was not shown in our studies ${ }^{16}$. Another analysis of Vlug et al. using the data of the LAFA trial showed that enforced advancement of oral intake, early mobilization, laparoscopic surgery and female sex were independent determinants of early recovery ${ }^{27}$. Another evaluation of implementation of an ERAS colon protocol and protocol compliance performed an univariable analysis which suggested that postoperative rather than preoperative and perioperative factors significantly influenced length of hospital stay ${ }^{24}$. The previous data might suggest that there are indeed several care elements that contribute more to recovery than others.

The influence of the degree of compliance to the ERAS protocol has also been examined. It is advocated that maximizing compliance to the various protocol is important to reach an optimal effect ${ }^{24}$. However, one study examining two groups managed in an ERAS pathway after colonic surgery showed that an overall protocol compliance of $77 \%$ does not worsen outcome as compared to a compliance of $88 \%{ }^{28}$. In 
another study evaluating an ERAS program in colorectal surgery, protocol compliance of less than $50 \%$ did show to deteriorate postoperative outcome (higher LOS, 30-day morbidity and readmission-rates) as compared to $70 \%, 80 \%$ or $90 \%$ compliance $^{29}$.

Compliance to an ERAS protocol in pancreatic surgery is a challenge. Pancreatic surgery still has high morbidity rates, and morbidity is an independent risk factor of a longer LOS as is shown in chapter 9. Furthermore, chapter 9 and 10 also showed that higher Dindo-Clavien grades correlated with longer LOS compared to complications with a lower Dindo-Clavien grade. In patients with a complicated postoperative course compliance to the postoperative items of the ERAS protocol is obviously less and sometimes even impossible. On the other hand, in uncomplicated patients this is easier and an LOS of 8-9 days as proposed in the ERAS protocol seems very well feasible. This is also shown by a recent retrospective study that reviewed 41 patients undergoing an uncomplicated PD. Twenty-one patients were managed in a fast track protocol and 20 patients received traditional care ${ }^{30}$. The LOS (including readmissions) was 9 days in the fast track group versus 14 in the traditional care group. Based on multivariable analysis, management in a fast track protocol was the only independent factor of a shorter LOS. This is in line with results shown in chapter 9. Unfortunately, compliance to protocol in this study with uncomplicated PD patients is not reported. A study of Robertson et al. managing 50 patients undergoing PD in a fast track protocol did report on protocol compliance and found it varying for different items between 36 and $82 \%$ with the most challenging items being early mobilization, the removal of drains and urine catheters. Similar compliance with the aforementioned items is also reported in chapter 10. LOS in this study was 9 days for uncomplicated patients. Importantly, a delay of discharge was reported for $32 \%$ of the patients, owing to social or transport-related reasons. So here also, functional recovery would have been a better readout.

The majority of studies reporting on ERAS programs for PD do not describe definitions for complications and do not use a standardized complication classification system. However, this is important for comparability between studies, especially when a metaanalysis on non-randomized studies is performed since such a meta-analysis is often limited by heterogeneity. We therefore used definitions for complications in our studies that were universally agreed on by the International Study Group of Pancreatic Fistula (ISGPF) and the International Study Group of Pancreatic Surgery (ISGPS) ${ }^{31-33}$. In addition, the well-validated Dindo-Clavien classification system for surgical complications was used in our studies ${ }^{14}$. The Dindo-Clavien classification aims to objectively group different complications with respect to the severity of therapeutic interventions and its use enhances comparability between studies.

In chapter 9, we described the outcome of patients undergoing PD in a 16-year time period in which the last 5 years patients were managed in an ERAS protocol. We noticed a reduction of 7 days in postoperative LOS in the ERAS group compared to the traditional care group. However, this decrease in LOS might not solely be attributable to the introduction of the ERAS program, since the increase in volume and 
accompanying experience with perioperative care probably also has had its influence. Several studies have reported improved outcome (lower morbidity and mortality rates and lower LOS) after PD proportional with volume and centralization ${ }^{34-37}$

In chapter 10 we evaluated elderly patients undergoing PD in an ERAS program. There is no official definition for the term "elderly", but most studies performed on pancreatic resections in the elderly population use a cut-off age above 70 years ${ }^{38-43}$. A recent analysis on a large cohort showed an age of 68.5 years can be considered as 'surgical oldness $^{44}$. We decided to define an interval centered on this age and consider older than 70 years as "elderly" and below 65 years as younger patients. Inevitably, a selection bias is introduced in this study by presenting only the fittest elderly with less severe co-morbidity for PD. Resection is the sole curative option for patients with malignant disease of the pancreas and even in the elderly the survival benefit after resection is not diminished with age ${ }^{45}$. However, the presence of risk factors and significant co-morbidities in the elderly result in lower overall survival rates and increased morbidity and mortality. Therefore it is very important to carefully select older patients and perform a meticulous pre-operative assessment. Older patients should be well informed about the risks and the fact that hospital volume and the experience of the surgeon play a significant role in the outcome ${ }^{35-37}$.

The main outcomes measured in studies about ERAS programs are LOS, morbidity and mortality rates. Patient perception and support are often overlooked but also essential for a successful implementation of an ERAS program. It has been proposed that patientreported quality of life has been adversely affected by fast track surgery with higher levels of emotional distress reported when compared with conventional surgery ${ }^{46}$. Also, difficulties with implementing ERAS programs are commonly encountered ${ }^{23,24,47}$. In order to gain more insight in patient and caregiver perceptions on the relative importance of the main components of an ERAS program we performed a multicenter international study (chapter 11). Anonymous web-based and paper surveys were sent to surgeons, anesthetists and nurses in centers in Scotland, Norway and the Netherlands. Additionally, pre-operative and post-operative surveys were completed by patients scheduled for major abdominal surgery in these institutions. Each questionnaire asked the responder to rate a selection of enhanced recovery principles and strategies in terms of perceived importance. 105 patients and 57 care providers were surveyed. Overall, patients and care providers found the majority of items important and supported ERAS principles. The highest rated items were: being free from nausea and free from pain at rest. The lowest ranked items were: being discharged from hospital as soon as possible and early restoration of gut function. The results of the survey also highlighted an area of concern for care providers, namely epidural use and fluid administration. These remain controversial issues amongst care providers and therefore have implications for future research. Furthermore, we identified several items scored low priority by care providers (e.g. avoidance of postoperative abdominal drains, oral bowel preparation and pre-anesthetic sedatives), for 
which there is a strong evidence base to the contrary. This underlines a potential barrier to ERAS implementation and is an area that requires consideration and education if continued sustainability and development of ERAS programs is to be achieved.

ERAS protocols are often seen as financially beneficial to institutions, particularly when analyzing the economic implications of reduced lengths of stay $^{48-50}$. However, our survey did not show patient or care provider support for reduced length of stay to be achieved as a priority over recovery. It highlighted that the priorities of patients and care providers are to avoid surgical complications and to achieve functional recovery rather than minimise their admission time in hospital. This poses a potential conflict between cost-analyzers and patients. Also, previous work has reported that enhanced recovery can increase anxiety following discharge ${ }^{46}$ and that patients who suffer a postoperative complication report more distress following early discharge than those with an uncomplicated recovery ${ }^{51}$. The results of our survey might therefore suggest that premature discharge is a potential source for anxiety in both patients and care providers.

Taking into account that there have been reports showing increased readmissions rates after an ERAS program ${ }^{52}$, post-discharge community surgical support is mandatory to lessen patient distress when attempting early discharge.

\section{FUTURE PERSPECTIVES}

\section{Implementation}

Despite a large amount of evidence to support the benefits of ERAS programs implementation of these programs into surgical practice is going slow. Several international surveys in Europe and the United States show that many institutions still do not perform evidence-based perioperative care in gastro-intestinal or HPB surgery $^{23,47,53-55}$. Problems with adopting guidelines in medicine has been described before in a large review ${ }^{56}$. Potential barriers to physician guideline adherence were lack of awareness and familiarity, lack of agreement, lack of self-efficacy and lack of the ability to overcome inertia of traditional practice. In addition, time limits and insufficient staff expertise may also play a role in slowing down the implementation of guidelines and fast-track programs. Better provision of information and multidisciplinary education about ERAS programs, especially in smaller institutions, can overcome these barriers. For this reason, the ERAS society has designed special implementation programs, which train perioperative teams to implement, reach and maintain high levels of compliance to the protocol elements (www.erassociety.org). Especially in the perioperative phase of the ERAS protocol many disciplines are involved. Consequently, a good organization and education for these disciplines is 
imperative to the success of the program. Moreover, junior nurses and registrars change units frequently, so continuous re-education is important ${ }^{24}$.

\section{Validation}

In chapter 6 we described the development of ERAS guidelines for PD. A framework of evidence-based recommendations for perioperative care for PD is presented. It forms the basis for future protocols. To assure the feasibility of this perioperative care protocol multinational and multicenter prospective validation in a large patient cohort is warranted. This also holds for the CEP for PD presented in chapter 5. An international $\mathrm{RCT}$ is now being initiated to evaluate the use of prophylactic intra-abdominal drains after PD and combines the aforementioned topics (chapter 7).

Patients undergoing PD who are included in this trial will be managed in the ERAS protocol as proposed by the ERAS group. Furthermore, this trial will contribute to the validation of the CEP for PD, as this CEP is used as an outcome measure. Additionally, to further validate the ERAS guidelines, multinational implementation of this consensus protocol has taken place from 2013 onwards in the departments of the members belonging to the ERAS group and will be evaluated in a prospective project separately.

\section{Patient perceptions}

The composite endpoint, which was developed for PD in chapter 5, did not include patient perceptions or quality of life measures as the respondents of the survey did not find these outcomes to be sufficiently PD-specific. Nevertheless, including patient perceptions in a CEP may be valuable since patients probably assign more weight to the consequences of certain complications. The OMERACT initiative (Outcome Measures in Rheumatoid Arthritis Clinical Trial) introduced the development of a Core Outcome Set (COS), this COS comprises a minimum of outcomes that should be reported in trials and it also includes patient perceptions ${ }^{57}$. More recently, the COMET initiative (Core Outcome Measures in Effectiveness Trials) extended this approach in order to develop COSs that are applicable to other disciplines as well, such as pediatrics and bariatric surgery $^{58,59}$. In addition to the CEP we are now developing a COS for pancreatic, liver and biliary surgery in collaboration with the COMET initiative.

\section{Specific interventions}

ERAS programs show promising results in improvements in outcome and reduction in LOS in many fields of surgery now. To achieve further improvement of outcomes research should be aimed at mediators that modify the stress response. As of yet there is still little information about which reactions in this stress response should be attenuated or stimulated. 
In hepatobiliary surgery, minimally invasive techniques are gaining popularity since these are known to reduce morbidity by diminishing catabolism and immunosuppression. Laparoscopic liver resections and even robotic liver resections are increasingly performed ${ }^{60-63}$. Recently, the first reports of laparoscopic PD have been published $^{64-66}$.

Pharmacological interventions have been proposed to attenuate the stress response. Perioperative $\beta$-blockage could have positive effects on postoperative mortality in highrisk patients ${ }^{67}$ and when morphine analgesia is provided during surgery, a selective morphine antagonist in the postoperative phase could reverse the negative effects of morphine on bowel function and attenuate postoperative ileus ${ }^{68}$. Glucocorticoids have been described to have an anti-inflammatory effect beneficial to outcome after major abdominal surgery. A meta-analysis of 11 RCTs found a decrease in complications and LOS when glucocorticoids were administered preoperatively ${ }^{69}$. Biological modifiers, such as neuropeptides, mast cell inhibitors and cytokine antagonists are promising pharmacological agents which improve postoperative immune function and diminish tissue inflammation ${ }^{70}$.

Additionally, advancement in analgesic and anesthetic techniques may also contribute to further improve fast-track surgery towards the ultimate goal: "creation of a stress and pain free operation with an enhanced recovery". 


\section{REFERENCES}

1. Adamina M, Kehlet H, Tomlinson GA, Senagore AJ, Delaney CP. Enhanced recovery pathways optimize health outcomes and resource utilization: a meta-analysis of randomized controlled trials in colorectal surgery. Surgery 2011;149: 830-840.

2. Varadhan KK, Neal KR, Dejong CH, Fearon KC, Ljungqvist O, Lobo DN. The enhanced recovery after surgery (ERAS) pathway for patients undergoing major elective open colorectal surgery: a meta-analysis of randomized controlled trials. Clin Nutr 2010; 29:434-440.

3. Vlug MS, Wind J, Hollmann MW, Ubbink DT, Cense HA, Engel AF, Gerhards MF, van Wagensveld BA, van der Zaag ES, van Geloven AA, Sprangers MA, Cuesta MA, Bemelman WA; LAFA study group. Laparoscopy in combination with fast track multimodal management is the best perioperative strategy in patients undergoing colonic surgery: a randomized clinical trial (LAFA-study). Ann Surg 2011;254: 868-875.

4. Ansari D, Gianotti L, Schröder J, Andersson R. Fast-track surgery: procedure-specific aspects and future direction. Langenbecks Arch Surg 2013;398:29-37.

5. Gurusamy KS, Samraj K, Davidson BR. Routine abdominal drainage for uncomplicated liver resection. Cochrane Database Syst Rev 2007:CD006232.

6. Petrowsky H, Demartines N, Rousson V, Clavien PA. Evidence-based value of prophylactic drainage in gastrointestinal surgery: a systematic review and meta-analyses. Ann Surg 2004;240:1074-1084;

7. Uetsuji S, Kwon AH, Komada H, Okuda Y, Imamura A, Kamiyama Y. Clinical evaluation of closed suction drainage following hepatectomy. Surg Today 1997; 27:298-301.

8. Diener MK, Tadjalli-Mehr K, Wente MN, Kieser M, Büchler MW, Seiler CM. Risk-benefit assessment of closed intra-abdominal drains after pancreatic surgery: a systematic review and meta-analysis assessing the current state of evidence. Langenbecks Arch Surg 2011;396:41-52.

9. Bassi C, Molinari E, Malleo G, Crippa S, Butturini G, Salvia R, Talamini G, Pederzoli P. Early versus late drain removal after standard pancreatic resections: results of a prospective randomized trial. Ann Surg 2010;252:207-214.

10. Kawai M, Tani M, Terasawa H, Ina S, Hirono S, Nishioka R, Miyazawa M, Uchiyama K, Yamaue H. Early removal of prophylactic drains reduces the risk of intra-abdominal infections in patients with pancreatic head resection: prospective study for 104 consecutive patients. Ann Surg 2006;244:1-7.

11. Kaido T. Analysis of randomized controlled trials on hepatopancreatic surgery. Dig Dis Sci 2006;51: 1761-1766.

12. Freemantle N1, Calvert M, Wood J, Eastaugh J, Griffin C. Composite outcomes in randomized trials: greater precision but with greater uncertainty? JAMA 2003;289:2554-2559.

13. Bethel MA1, Holman R, Haffner SM, Califf RM, Huntsman-Labed A, Hua TA, McMurray J. Determining the most appropriate components for a composite clinical trial outcome. Am Heart J 2008;156:633-640.

14. Clavien PA, Barkun J, de Oliveira ML, Vauthey JN, Dindo D, Schulick RD, de Santibañes E, Pekolj J, Slankamenac K, Bassi C, Graf R, Vonlanthen R, Padbury R, Cameron JL, Makuuchi M. The Clavien-Dindo classification of surgical complications: five-year experience. Ann Surg 2009;250:187-196.

15. Ferreira-González I, Busse JW, Heels-Ansdell D, Montori VM, Akl EA, Bryant DM, Alonso-Coello P, Alonso J, Worster A, Upadhye S, Jaeschke R, Schünemann HJ, Permanyer-Miralda G, Pacheco-Huergo V, Domingo-Salvany A, Wu P, Mills EJ, Guyatt GH. Problems with use of composite end points in cardiovascular trials: systematic review of randomised controlled trials. BMJ 2007;334:786.

16. Balzano G, Zerbi A, Braga M, Rocchetti S, Beneduce AA, Di Carlo V. Fast-track recovery programme after pancreatico- duodenectomy reduces delayed gastric emptying. Br J Surg 2008;95:1387-1393.

17. di Sebastiano P, Festa L, De Bonis A, Ciuffreda A, Valvano MR, Andriulli A, di Mola FF. A modified fasttrack program for pancreatic surgery: a prospective single-center experience. Langenbecks Arch Surg 2011;396:345-351.

18. Kennedy EP, Rosato EL, Sauter PK, Rosenberg LM, Doria C, Marino IR, Chojnacki KA, Berger AC, Yeo CJ. Initiation of a critical pathway for pancreaticoduodenectomy at an academic institution--the first step in multidisciplinary team building. J Am Coll Surg 2007;204:917-923; .

19. Berberat PO, Ingold H, Gulbinas A, Kleeff J, Müller MW, Gutt C, Weigand M, Friess H, Büchler MW. Fast track--different implications in pancreatic surgery. J Gastrointest Surg 2007; 11:880-887. 
20. Maessen JM, Dejong CH, Kessels AG, von Meyenfeldt MF; Enhanced Recovery After Surgery (ERAS) Group. Length of stay: an inappropriate readout of the success of enhanced recovery programs. World J Surg 2008;32:971-975.

21. Campbell M, Fitzpatrick R, Haines A, Kinmonth AL, Sandercock $P$, Spiegelhalter D, Tyrer P. Framework for design and evaluation of complex interventions to improve health. BMJ 2000;321:694-696.

22. Nygren J, Hausel J, Kehlet H, Revhaug A, Lassen K, Dejong C, Andersen J, von Meyenfeldt M, Ljungqvist $\mathrm{O}$, Fearon KC. A comparison in five European Centres of case mix, clinical management and outcomes following either conventional or fast-track perioperative care in colorectal surgery. Clin Nutr 2005;24: 455-461.

23. Lassen K, Hannemann P, Ljungqvist O, Fearon K, Dejong CH, von Meyenfeldt MF, Hausel J, Nygren J, Andersen J, Revhaug A; Enhanced Recovery After Surgery Group. Patterns in current perioperative practice: survey of colorectal surgeons in five northern European countries. BMJ 2005;330:1420-1421.

24. Maessen J, Dejong CH, Hausel J, Nygren J, Lassen K, Andersen J, Kessels AG, Revhaug A, Kehlet H, Ljungqvist $\mathrm{O}$, Fearon $\mathrm{KC}$, von Meyenfeldt MF. A protocol is not enough to implement an enhanced recovery programme for colorectal resection. Br J Surg 2007;94:224-231.

25. Stoot JH, van Dam RM, Busch OR, van Hillegersberg R, De Boer M, Olde Damink SW, Bemelmans MH, Dejong $\mathrm{CH}$; Enhanced Recovery After Surgery (ERAS) Group. The effect of a multimodal fast-track programme on outcomes in laparoscopic liver surgery: a multicentre pilot study. HPB (Oxford) 2009;11:140-144.

26. Gillissen F, Hoff C, Maessen JM, Winkens B, Teeuwen JH, von Meyenfeldt MF, Dejong CH. Structured synchronous implementation of an enhanced recovery program in elective colonic surgery in 33 hospitals in The Netherlands. World J Surg 2013;37:1082-1093.

27. Vlug MS, Bartels SA, Wind J, Ubbink DT, Hollmann MW, Bemelman WA; Collaborative LAFA Study Group. Which fast track elements predict early recovery after colon cancer surgery? Colorectal Dis 2012;14:1001-1008.

28. Ahmed J, Khan S, Gatt M, Kallam R, MacFie J. Compliance with enhanced recovery programmes in elective colorectal surgery. Br J Surg 2010;97:754-758.

29. Gustafsson UO, Hausel J, Thorell A, Ljungqvist O, Soop M, Nygren J; Enhanced Recovery After Surgery Study Group. Adherence to the enhanced recovery after surgery protocol and outcomes after colorectal cancer surgery. Arch Surg 2011;146:571-577.

30. Nikfarjam M1, Weinberg L, Low N, Fink MA, Muralidharan V, Houli N, Starkey G, Jones R, Christophi C. A fast track recovery program significantly reduces hospital length of stay following uncomplicated pancreaticoduodenectomy. JOP 2013;14:63-70.

31. Wente MN1, Veit JA, Bassi C, Dervenis C, Fingerhut A, Gouma DJ, Izbicki JR, Neoptolemos JP, Padbury RT, Sarr MG, Yeo CJ, Büchler MW. Postpancreatectomy hemorrhage (PPH): an International Study Group of Pancreatic Surgery (ISGPS) definition. Surgery 2007;142:20-25.

32. Wente MN1, Bassi C, Dervenis C, Fingerhut A, Gouma DJ, Izbicki JR, Neoptolemos JP, Padbury RT, Sarr MG, Traverso LW, Yeo CJ, Büchler MW. Delayed gastric emptying (DGE) after pancreatic surgery: a suggested definition by the International Study Group of Pancreatic Surgery (ISGPS). Surgery 2007; 142:761-768.

33. Bassi C, Dervenis C, Butturini G, Fingerhut A, Yeo C, Izbicki J, Neoptolemos J, Sarr M, Traverso W, Buchler M; International Study Group on Pancreatic Fistula Definition. Postoperative pancreatic fistula: an international study group (ISGPF) definition. Surgery 2005;138:8-13.

34. de Wilde RF, Besselink MG, van der Tweel I, de Hingh IH, van Eijck CH, Dejong CH, Porte RJ, Gouma DJ, Busch OR, Molenaar IQ; Dutch Pancreatic Cancer Group. Impact of nationwide centralization of pancreaticoduodenectomy on hospital mortality. Br J Surg 2012;99:404-410.

35. Balzano G, Zerbi A, Capretti G, Rocchetti S, Capitanio V, Di Carlo V. Effect of hospital volume on outcome of pancreaticoduodenectomy in Italy. Br J Surg 2008;95:357-362.

36. Gouma DJ, van Geenen RC, van Gulik TM, de Haan RJ, de Wit LT, Busch OR, Obertop H. Rates of complications and death after pancreaticoduodenectomy: risk factors and the impact of hospital volume. Ann Surg 2000;232:786-795.

37. Riall TS. What is the effect of age on pancreatic resection? Adv Surg 2009;43:233-249.

38. al-Sharaf K, Andren-Sandberg A, Ihse I Subtotal pancreatectomy for cancer can be safe in the elderly. Eur J Surg 1999;165:230-235. 
39. Bottger TC, Engelmann R, Junginger T. Is age a risk factor for major pancreatic surgery? An analysis of 300 resections. Hepatogastroenterology 1999;46:2589-2598.

40. Brozzetti S, Mazzoni G, Miccini M, Puma F, De Angelis M, Cassini D, Bettelli E, Tocchi A, Cavallaro A. Surgical treatment of pancreatic head carcinoma in elderly patients. Arch Surg 2006; 141:137-142.

41. Fong Y, Blumgart LH, Fortner JG, Brennan MF. Pancreatic or liver resection for malignancy is safe and effective for the elderly. Ann Surg 1995;222:426-434; discussion 434-437.

42. Hodul P, Tansey J, Golts E, Oh D, Pickleman J, Aranha GV. Age is not a contraindication to pancreaticoduodenectomy. Ann Surg 2001;67:270-275;.

43. Scurtu R, Bachellier P, Oussoultzoglou E, Rosso E, Maroni R, Jaeck D. Outcome after pancreaticoduodenectomy for cancer in elderly patients. J Gastrointest Surg 2006;10:813-822.

44. Kurian AA, Wang L, Grunkemeier G, Bhayani NH, Swanström LL. Defining "the elderly" undergoing major gastrointestinal resections: receiver operating characteristic analysis of a large ACS-NSQIP cohort. Ann Surg 2013;258:483-489.

45. Finlayson E, Fan Z, Birkmeyer JD. Outcomes in octogenarians undergoing high-risk cancer operation: a national study. J Am Coll Surg 2007;205:729-734.

46. Wennström B, Stomberg MW, Modin M, Skullman S. Patient symptoms after colonic surgery in the era of enhanced recovery--a long-term follow-up. J Clin Nurs 2010;19:666-672.

47. Wong-Lun-Hing EM, Lodewick TM, Stoot JH, Bemelmans $\mathrm{MH}$, Olde Damink SW, Dejong $\mathrm{CH}$, van Dam RM. A survey in the hepatopancreatobiliary community on ways to enhance patient recovery. $H P B$ (Oxford) 2012;14:818-827.

48. Vanounou T, Pratt W, Fischer JE, Vollmer CM Jr, Callery MP. Deviation-based cost modeling: a novel model to evaluate the clinical and economic impact of clinical pathways. J Am Coll Surg 2007;204: 570-579.

49. Lemanu DP, Singh PP, Berridge K, Burr M, Birch C, Babor R, MacCormick AD, Arroll B, Hill AG. Randomized clinical trial of enhanced recovery versus standard care after laparoscopic sleeve gastrectomy. Br J Surg 2013;100:482-489.

50. Porter GA, Pisters PW, Mansyur C, Bisanz A, Reyna K, Stanford P, Lee JE, Evans DB. Cost and utilization impact of a clinical pathway for patients undergoing pancreaticoduodenectomy. Ann Surg Oncol 2000;7:484-489.

51. Blazeby JM, Soulsby M, Winstone K, King PM, Bulley S, Kennedy RH. A qualitative evaluation of patients' experiences of an enhanced recovery programme for colorectal cancer. Colorectal Dis 2010;12: e236-242.

52. Nygren J, Soop M, Thorell A, Hausel J, Ljungqvist O; ERAS Group. An enhanced-recovery protocol improves outcome after colorectal resection already during the first year: a single-center experience in 168 consecutive patients. Dis Colon Rectum 2009; 52:978-985.

53. Slim K, Panis Y, Chipponi J; Société Française de Chirurgie Digestive. Half of the currecnt practice of gastrointestinal surgery is against the evidence: a survery of the French Society of Digestive Surgery. $J$ Gastrointest Surg 2004;8:1079-1082.

54. Hannemann P, Lassen K, Hausel J, Nimmo S, Ljungqvist O, Nygren J, Soop M, Fearon K, Andersen J, Revhaug $\mathrm{A}$, von Meyenfeldt MF, Dejong $\mathrm{CH}$, Spies C. Patterns in current anaesthesiological perioperative practice for colonic resections: a survey in five northern-European countries. Acta Anaesthesiol Scand 2006;50:1152-1160.

55. Walter CJ, Smith A, Guillou P. Perceptions of the application of fast-track surgical principles by general surgeons. Ann R Coll Surg Engl 2006;88:191-195.

56. Cabana MD, Rand CS, Powe NR, Wu AW, Wilson MH, Abboud PA, Rubin HR. Why don't physicians follow clinical practice guidelines? A framework for improvement. JAMA 1999;282:1458-1465.

57. Tugwell P, Boers M, Brooks P, Simon L, Strand V, Idzerda L. OMERACT: an international initiative to improve outcome measurement in rheumatology. Trials 2007;8:38.

58. Sinha IP, Gallagher R, Williamson PR, Smyth RL. Development of a core outcome set for clinical trials in childhood asthma: a survey of clinicians, parents, and young people. Trials 2012;13:103.

59. Available from: http://blogs.bmj.com/bmj/2012/08/01/james-hopkins-and-jane-blazeby-developmentof-a-core-outcome-set-for-bariatric-surgery/.

60. Kirchberg J, Reißfelder C, Weitz J, Koch M. Laparoscopic surgery of liver tumors. Langenbecks Arch Surg 2013;398:931-938. 
61. Zhou Y, Xiao Y, Wu L, Li B, Li H. Laparoscopic liver resection as a safe and efficacious alternative to open resection for colorectal liver metastasis: a meta-analysis. BMC Surg 2013;13:44.

62. Boggi U, Caniglia F, Amorese G. Laparoscopic robot-assisted major hepatectomy. J Hepatobiliary Pancreat Sci 2013.

63. Stoot JH, Wong-Lun-Hing EM, Limantoro I, Visschers R, Busch OR, Van Hillegersberg R, De Jong KM, Rijken AM, Kazemier G, Olde Damink SW, Lodewick TM, Bemelmans MH, van Dam RM, Dejong $\mathrm{CH}$; Dutch Liver Collaborative Group. Laparoscopic liver resection in the Netherlands: how far are we? Dig Surg 2012;29:70-78.

64. Kendrick ML, Cusati D. Total laparoscopic pancreaticoduodenectomy: feasibility and outcome in an early experience. Arch Surg 2010;145:19-23.

65. Jacobs MJ, KamyabA. Total laparoscopic pancreaticoduodenectomy. JSLS 2013;17:188-193.

66. Gagner M, Palermo M. Laparoscopic Whipple procedure: review of the literature. J Hepatobiliary Pancreat Surg 2009;16:726-730.

67. Poldermans D, Boersma E, Bax JJ, Thomson IR, van de Ven LL, Blankensteijn JD, Baars HF, Yo TI, Trocino $G$, Vigna $C$, Roelandt JR, van Urk $H$. The effect of bisoprolol on perioperative mortality and myocardial infarction in high-risk patients undergoing vascular surgery. Dutch Echocardiographic Cardiac Risk Evaluation Applying Stress Echocardiography Study Group. N Engl J Med 1999;341:1789-1794.

68. Taguchi A, Sharma N, Saleem RM, Sessler DI, Carpenter RL, Seyedsadr M, Kurz A. Selective postoperative inhibition of gastrointestinal opioid receptors. N Engl J Med 2001;345:935-940.

69. Srinivasa S, Kahokehr AA, Yu TC, Hill AG. Preoperative glucocorticoid use in major abdominal surgery: systematic review and meta-analysis of randomized trials. Ann Surg 2011;254:183-191.

70. Hoffmann $\mathrm{H}$, Kettelhack $\mathrm{C}$. Fast-track surgery--conditions and challenges in postsurgical treatment: a review of elements of translational research in enhanced recovery after surgery. Eur Surg Res 2012;49:24-34. 
Short summary 


\section{SHORT SUMMARY}

Liver and pancreatic resections are complex major abdominal surgical procedures that are still associated with considerable morbidity (up to 50\%) and mortality rates (2-4\%). Traditional perioperative care for liver and pancreatic surgery has been conservative and dogmatic. Furthermore, evidence-based techniques and care elements have not yet been implemented extensively in this field. In this thesis we aimed to develop and implement an evidence-based "Enhanced Recovery After Surgery" (ERAS) care protocol in liver and pancreatic surgery in order to diminish the stress response to these major abdominal resections and accelerate recovery without compromising outcome.

In the past, ERAS programs have been implemented in colonic surgery. Multiple trials showed that these programs accelerate recovery and decrease morbidity in patients undergoing colonic resections. Additionally, hospital costs were shown to be reduced. However, despite the successes in colonic surgery, it is unclear whether an ERAS protocol can also be successfully implemented in major abdominal surgery, such as liver and pancreatic resections. It was the main purpose of this thesis to investigate whether this is indeed possible.

We started our research by conducting a systematic review and meta-analysis to evaluate the feasibility of ERAS programs in liver and pancreatic surgery. We concluded that there is some evidence for the effectiveness of an ERAS program in liver and pancreatic surgery. However, the number of high quality studies is low and protocols differ considerably amongst trials. Also, there is great variability in the endpoints and definitions used. For this reason, there is a need for uniform definitions and a core set of outcomes that should be reported in each study. We performed a systematic review and a web-survey amongst experts in hepato-pancreatico-biliary units. In this study we proposed definitions and a core outcome set as a composite endpoint. All experts reached consensus about using these definitions and the composite endpoint in future trials.

Subsequently, a uniform evidence-based ERAS protocol for pancreaticoduodenectomy was developed in collaboration with the ERAS group (www.erassociety.org) and proposed for further use. In this protocol consensus was reached on several items, but no consensus was reached on the use of prophylactic abdominal drainage. We therefore conducted a meta-analysis into this matter, which provided no conclusive evidence either. Subsequently we designed a multicenter, international randomized controlled trial, which examines the necessity of routine placement of a prophylactic abdominal drain after pancreaticoduodenectomy. This study is still ongoing.

Then we evaluated the implementation of an ERAS program in liver surgery and pancreatic surgery. We concluded that implementing an ERAS program in both forms of surgery is feasible with a decrease of postoperative length of stay without increasing morbidity, mortality or readmission rates. In addition, implementation of such an ERAS protocol in elderly patients undergoing pancreaticoduodenectomy seems feasible and 
safe as well. However, adherence to the ERAS program becomes more difficult in case of complications. Finally, we assessed care providers' and patients' perceptions of the relevance and importance of the ERAS principles and concluded that ERAS principles are supported by patients and care providers. Patients and care providers rank functional recovery higher than reduced length of stay.

To conclude, we developed, implemented and evaluated an ERAS program in liver and pancreatic surgery and found that an ERAS program in these forms of surgery is feasible and does not compromise outcomes. However, morbidity remains high and efforts should be made to examine ways to further reduce complications rates and to increase protocol adherence. 
Summary in Dutch 


\section{SUMMARY IN DUTCH}

Lever en pancreas resecties zijn complexe en grote abdominale chirurgische procedures met een hoge morbiditeit (tot 50\%) en tegenwoordig een acceptabele mortaliteit (2$4 \%)$. Traditionele perioperatieve zorg voor lever en pancreas chirurgie is conservatief en dogmatisch. Evidence-based technieken en zorgelementen zijn niet geïmplementeerd in deze vormen van chirurgie. Met dit proefschrift beogen we een versneld herstel programma na lever en pancreas chirurgie (ERAS) te ontwikkelen, implementeren en evalueren met als doel de stress respons na deze grote abdominale procedures te verminderen en het herstel te versnellen zonder dat andere uitkomstmaten gecompromitteerd worden.

ERAS programma's zijn al eerder ontwikkeld en geïmplementeerd in colonchirurgie. Meerdere trials laten zien dat een ERAS programma voor patiënten die een colonresectie ondergaan het herstel versnelt en morbiditeit doet afnemen. Tevens zijn de ziekenhuiskosten verminderd wanneer een ERAS programma gevolgd wordt. Ondanks het succes van deze programma's in de colonchirurgie, is het echter nog onduidelijk of een ERAS protocol ook kan worden geïmplementeerd in grotere abdominale ingrepen, zoals lever en pancreas chirurgie. Het voornaamste doel van dit proefschrift is om te onderzoeken of het implementeren van een ERAS programma in lever en pancreas chirurgie inderdaad mogelijk is.

We hebben ons onderzoek gestart met het verrichten van een systematisch review en meta-analyse om te haalbaarheid van ERAS programma's in lever en pancreas chirurgie te evalueren. We concludeerden dat er wel enig bewijs is voor de effectiviteit van ERAS programma's in lever en pancreas chirurgie. Echter, het aantal studies van hoge kwaliteit is laag en de aard van de geïmplementeerde ERAS protocollen verschilt behoorlijk tussen de studies. Er is ook een grote variabiliteit in de eindpunten en definities die voor complicaties gebruikt worden. Het is voor de vergelijkbaarheid van studies noodzakelijk om uniforme definities te gebruiken en een vaste set van uitkomsten zou beschreven moeten worden in elke studie. We verrichtten een systematisch review en online onderzoek onder experts die werkzaam zijn op hepatopancreatico-biliare afdelingen. In deze studie stelden we definities voor specifieke complicaties voor en een vaste set van uitkomsten als één samengesteld eindpunt. $\mathrm{Er}$ werd door de experts consensus bereikt over deze definities en het samengesteld eindpunt, dat nu gebruikt kan worden in toekomstige trials.

Vervolgens ontwikkelden wij in samenwerking met de ERAS groep (www.erassociety.org) een evidence-based ERAS protocol voor pancreaticoduodenectomie. In dit protocol werd over een aantal zaken consensus bereikt, er werd echter geen consensus bereikt over het al dan niet achterlaten van een profylactische abdominale drain. Om nogmaals alle bewijs hiervoor kritisch te bekijken, verrichtten wij een meta-analyse. Dit gaf ons echter geen uitsluitsel. Daarom ontworpen we een nieuwe internationale multicenter gerandomiseerde trial, die de noodzaak van 
routinematig profylactisch abdominaal draineren na een pancreaticoduodenectomie gaat onderzoeken. Deze studie is gestart en loopt op dit moment nog.

Hierna evalueerden we de implementatie van een ERAS programma in lever en pancreas chirurgie. We concludeerden dat het implementeren van een ERAS programma in beide vormen van chirurgie haalbaar is met een afname van de postoperatieve opname duur zonder dat de morbiditeit, mortaliteit en heropnames zijn toegenomen. Tevens blijkt de implementatie van een ERAS programma voor ouderen die een pancreaticoduodenectomie ondergaan haalbaar. Echter, het volgen van het ERAS protocol is moeilijker wanneer er complicaties optreden. Ten slotte beoordeelden we de percepties van patiënten en zorgverstrekkers over de relevantie en het belang van specifieke ERAS principes. We concludeerden dat de ERAS principes worden gesteund door zowel patiënten als zorgverstrekkers. Het blijkt dat patiënten en zorgverstrekkers het bereiken van functioneel herstel belangrijker vinden dan een snel ontslag uit het ziekenhuis.

Concluderend hebben we een ERAS programma ontwikkeld, geïmplementeerd en geëvalueerd in lever en pancreas chirurgie en vonden we dat een ERAS programma haalbaar is voor beide vormen van chirurgie zonder dat de andere uitkomstmaten gecompromitteerd worden. Echter, de morbiditeitscijfers blijven nog steeds hoog. We zouden daarom verder moeten onderzoeken hoe we de complicaties kunnen verminderen en de mate van precies volgen van het ERAS protocol kunnen vergroten. 
Valorization 


\section{VALORIZATION}

\section{INTRODUCTION}

In this thesis we aimed to implement Enhanced Recovery After Surgery (ERAS) programs in liver and pancreatic surgery. In short, an ERAS program addresses a variety of evidence-based perioperative interventions and demands a multidisciplinary approach in which surgeons, anesthesiologists, intensive care staff, and nurses work together as a team. The purpose of an ERAS program is to accelerate postoperative recovery and shorten postoperative length of stay (LOS) without an increased in or even with a lower morbidity and readmission rate. Perioperative management in an ERAS program has a major impact on the patient and his social environment.

Until recently patients undergoing liver or pancreatic surgery were not allowed to eat postoperatively, since surgeons thought this might compromise recent anastomoses and induce delayed gastric emptying. Patients were summoned to stay in bed to rest and drains in the abdomen were routinely placed after liver and pancreatic surgery. The ERAS program cares to provide better pre-operative information to the patient and allows patients to restart intake and mobilize directly after surgery. It has been shown that this does not harm the patient. On the contrary, the incidence of complications decreased (as shown in colonic surgery) and LOS was reduced after implementing an ERAS program in many surgical fields.

\section{DUTCH REGISTRIES}

Dutch registries for liver and pancreatic surgical treatment (Dutch Hepato Biliary Audit (DHBA) and Dutch Pancreatic Cancer Audit (DPCA) $)^{1,2}$ aim to register results of benign and malignant pancreatic and liver surgery throughout the Netherlands and give insight in quality of care in different institutes. Comparing results within the Netherlands and with other countries allows for developing the best treatment strategies and improve perioperative care. It is therefore important for Dutch hospitals and HPB departments to perform well. Consequently, ERAS programs are becoming more interesting for HPB groups since it has been shown (in this thesis) that implementation of an ERAS program improves care and decreases $\operatorname{LOS}^{3,4}$. This leads to better results in the registries and encourages other departments to implement ERAS programs as well.

\section{COST-EFFECTIVENESS}

Health care costs in the Netherlands are rising each year with an expected increase of $5.3 \%$ in 2014 leading to a total of 1.97 billion euros $^{5}$. It is therefore of utmost importance to control and cut down these costs whenever possible. Use of an ERAS program contributes significantly to reducing such health care costs. 
Originally, ERAS programs were designed to reduce postoperative stress and accelerate recovery. However, many studies have shown that an ERAS program for HPB surgery also significantly reduces in-hospital expenses (Chapter 2 of this thesis) ${ }^{3}$. As an example, a study of Kennedy et al. on patients undergoing pancreaticoduodenectomy demonstrated that hospital costs after implementing an ERAS program were almost halved (from $\$ 240,242$ to $\$ 126,566$ per patient, $p<0.001)^{6}$. This dramatic decrease in costs is mainly due to the streamlining of care and decrease in postoperative hospital stay displayed in most studies. Thus, ERAS programs are very valuable when it comes to cost-effective use of health care. This is obviously favorable to health insurance companies as well. As Kennedy stated: "ERAS programs are one way to meeting the challenges of the oncoming 'pay to performance' era" ${ }^{6}$.

\section{PATIENTS AND EVIDENCE-BASED CARE}

Patients are more demanding nowadays and expect the best possible care. In addition, surgical care is increasingly demanded to be evidence-based. For this reason it currently seems unethical to subject patients to traditional non-evidence-based care. The ERAS program combines perioperative interventions that are all evidence-based and its implementation should consequently lead to better surgical care. Patients benefit from better perioperative care and accelerated recovery.

\section{IMPLEMENTATION}

Despite a large amount of evidence to support the benefits of ERAS programs, implementation of these programs into surgical practice is going slow. Several international surveys in Europe and the United States show that many institutions still do not adhere to evidence-based perioperative care in gastro-intestinal or HPB surgery $^{7-10}$. Problems with adopting guidelines in medicine have been described earlier in a large review ${ }^{11}$. Potential barriers to (physician) guideline adherence were lack of awareness and familiarity, lack of agreement, lack of self-efficacy and lack of the ability to overcome inertia of traditional practice. In addition, time limits and insufficient staff expertise may also play a role in slowing down the implementation of guidelines and fast-track programs. Better provision of information and multidisciplinary education about ERAS programs, especially in smaller institutions, can overcome these barriers. For this reason, the ERAS society has designed special implementation programs, which train perioperative teams to implement, reach and maintain high levels of compliance to the protocol elements (www.erassociety.org). Especially in the perioperative phase of the ERAS protocol many disciplines are involved. Consequently, a good organization and education for these disciplines is imperative to the success of the program. Moreover, junior nurses and registrars change units frequently, so continuous re-education is important ${ }^{12}$. 


\section{INNOVATION}

Kehlet and Wilmore were the first to describe and implement an ERAS protocol successfully in colonic surgery ${ }^{13}$. Subsequently, numerous studies have been published on this topic, not only in colonic surgery but also in many other fields of surgery (e.g., musculoskeletal $^{14}$, breast $^{15}$, aortic ${ }^{16,17}$, bariatric ${ }^{18,19}$, and prostate surgery ${ }^{20}$ ). All of these studies showed a decrease in LOS, without increasing postoperative morbidity or mortality. Despite this, there is relatively little experience with ERAS programs for liver and pancreatic surgery. Wong-Lun-Hing et al. conducted a worldwide survey amongst 673 hepatobiliary centers (the response rate was 75\%) and collected information the centers' experience with ERAS programs in different fields of surgery ${ }^{10}$. Only $17.4 \%$ of the centers had experience with an ERAS program for pancreatic surgery and $28 \%$ of the centers had experience with an ERAS program for liver surgery. As a comparison, for colonic surgery nearly $40 \%$ of the centers had experience with an ERAS program (Table 1). In conclusion, the adoption of ERAS programs for liver and pancreatic surgery worldwide is still limited. By developing the ERAS consensus-based guidelines for pancreaticoduodenectomy (PD) the ERAS group (which we are member of) leads the field.

Table 1 Centers with experience in fast-track perioperative care programs.

\begin{tabular}{lrrrr}
\hline Region & \multicolumn{3}{c}{ Experience with ERAS programs in a specific type of surgery, $n /$ total $n$ (\%) } \\
& No experience & In colon surgery & In liver surgery & In pancreatic surgery \\
\hline Europe & $31 / 72(43.1 \%)$ & $36 / 72(50.0 \%)$ & $22 / 72(30.6 \%)$ & $14 / 72(19.4 \%)$ \\
North America & $25 / 42(59.5 \%)$ & $6 / 42(14.3 \%)$ & $9 / 42(21.4 \%)$ & $8 / 42(19.0 \%)$ \\
Central and South America & $5 / 10(50.0 \%)$ & $4 / 10(40.0 \%)$ & $3 / 10(30.0 \%)$ & $1 / 10(10.0 \%)$ \\
Asia & $15 / 24(62.5 \%)$ & $8 / 24(33.3 \%)$ & $4 / 24(16.7 \%)$ & $3 / 24(12.5 \%)$ \\
Oceania & $2 / 8(25.0 \%)$ & $5 / 8(62.5 \%)$ & $6 / 8(75.0 \%)$ & $2 / 8(25.0 \%)$ \\
Africa & $4 / 5(80.0 \%)$ & $1 / 5(20.0 \%)$ & $1 / 5(20.0 \%)$ & $0 / 5(0 \%)$ \\
Worldwide & $82 / 161(50.9 \%)$ & $50 / 161(37.3 \%)$ & $45 / 161(28.0 \%)$ & $28 / 161(17.4 \%)$ \\
\hline
\end{tabular}

ERAS, enhanced recovery after surgery.

\section{PLANNING AND REALISATION}

In chapter 6 we described the development of ERAS guidelines for pancreaticoduodenectomy $(P D)^{21,22}$. A framework of evidence-based recommendations for perioperative care for PD is presented. It forms the basis for future protocols. To assure the feasibility of this perioperative care protocol multinational and multicenter prospective validation in a large patient cohort is warranted. This also holds for the composite endpoint (CEP) for PD presented in chapter $5^{23}$. An international randomized controlled trial (RCT) is now being initiated to evaluate the use of prophylactic intra-abdominal drains after PD and combines the aforementioned topics (chapter 7, NTR3224). Patients undergoing PD who are included in this trial will be managed in the ERAS protocol as proposed by the ERAS group. Furthermore, this trial will contribute to the validation of the CEP for PD, as this CEP is used as an outcome measure. 
Additionally, to further validate the ERAS guidelines, multinational implementation of this consensus protocol has taken place from 2013 onwards in the departments of the members belonging to the ERAS group and will be evaluated in a prospective project separately.

Following the ERAS guidelines for PD, the ERAS group is now also working on guidelines for liver surgery. With the development of such guidelines we hope to achieve uniform and optimal perioperative care worldwide with favorable outcome for all of our patients.

In chapter 8, 9 and 10 we present outcomes after implementing an ERAS program for liver and pancreatic surgery. Length of stay (LOS) after liver surgery decreased with two days (from 8 to 6 days, $p<0.001)$ ) and LOS after pancreatic surgery decreased significantly from 20 days (1995-2005) to 14 days (2009-2011), $p<0.05$ (versus 16 to 9 days for patients being discharged without complications, $p<0.05$ ). However, several publications have shown that LOS is influenced by several confounding factors and might therefore not the best outcome parameter. Time to functional recovery or readiness for discharge is probably a better readout. Maessen et al. showed that $87 \%$ of the patients undergoing colonic surgery in an ERAS program were not discharged on the day that discharge criteria were fulfilled ${ }^{24}$. Fiore et al. published similar results ${ }^{25}$. Reasons for not being discharged were 'surgeons judgment', absence of sufficient support or planning, and patients required (more) training for stoma management. The authors encourage the use of time to readiness for discharge as a measure of shortterm recovery. Our group already published a cohort of patients undergoing laparoscopic liver surgery in an ERAS program and found that time to functional recovery was reached 2 days before the actual discharge ${ }^{26}$. In our cohort of patients undergoing liver and pancreatic surgery we did not specifically look at time to functional recovery, but the estimated difference between time to functional recovery and actual discharge should be similar in the patients undergoing liver surgery. For patients undergoing pancreatic surgery it might be even more, since surgeons are more cautious to discharge patients early after a PD, even when discharge criteria are met, because of the possible occurrence of late complications such as hemorrhage or pancreatic fistula.

Maessen et al. and Gillissen et al. presented the results of a 'Dutch breakthrough' project in which 33 Dutch hospitals signed up to implement an ERAS program for colonic surgery ${ }^{27,28}$. This was a successful project in all hospitals resulting in ERAS patients eating 3 days earlier than patients treated in traditional care. Overall LOS was decreased from 9 to 6 days and functional recovery was achieved after 3 days. Now that we have gathered sufficient evidence for the implementation of ERAS programs in liver and pancreatic surgery, a national breakthrough project (such as the recent project for ERAS in colonic surgery) might be the way forward. 


\section{REFERENCES}

1. http://dhba.clinicalaudit.nl/over-dhba.

2. http://dpca.clinicalaudit.nl/.

3. Coolsen MM, van Dam RM, van der Wilt AA, Slim K, Lassen K, Dejong CH. Systematic review and metaanalysis of enhanced recovery after pancreatic surgery with particular emphasis on pancreaticoduodenectomies. World J Surg. 2013;37:1909-1918.

4. Coolsen MM, Wong-Lun-Hing EM, van Dam RM, et al. A systematic review of outcomes in patients undergoing liver surgery in an enhanced recovery after surgery pathways. HPB (Oxford) 2013;15: 245-251.

5. http://www.zorgvisie.nl/Financien/Nieuws/2013/11/Verzekeraars-zien-zorgkosten-flink-stijgen$1401961 \mathrm{~W} /$.

6. Kennedy EP, Rosato EL, Sauter PK, et al. Initiation of a critical pathway for pancreaticoduodenectomy at an academic institution--the first step in multidisciplinary team building. J Am Coll Surg 2007;204: 917-923.

7. Lassen $\mathrm{K}$, Hannemann $\mathrm{P}$, Ljungqvist $\mathrm{O}$, et al. Patterns in current perioperative practice: survey of colorectal surgeons in five northern European countries. BMJ 2005;330:1420-1421.

8. Slim K, Panis Y, Chipponi J, Societe Francaise de Chirurgie D. Half of the currecnt practice of gastrointestinal surgery is against the evidence: a survery of the French Society of Digestive Surgery. $J$ Gastrointest Surg 2004;8:1079-1082.

9. Walter CJ, Smith A, Guillou P. Perceptions of the application of fast-track surgical principles by general surgeons. Ann R Coll Surg Engl 2006;88:191-195.

10. Wong-Lun-Hing EM, Lodewick TM, Stoot $\mathrm{JH}$, et al. A survey in the hepatopancreatobiliary community on ways to enhance patient recovery. HPB (Oxford) 2012;14:818-827.

11. Cabana MD, Rand CS, Powe NR, et al. Why don't physicians follow clinical practice guidelines? A framework for improvement. JAMA 1999;282:1458-1465.

12. Maessen J, Dejong $\mathrm{CH}$, Hausel J, et al. A protocol is not enough to implement an enhanced recovery programme for colorectal resection. Br J Surg 2007;94:224-231.

13. Kehlet H, Wilmore DW. Multimodal strategies to improve surgical outcome. Am J Surg 2002;183: 630-641.

14. Barbieri A, Vanhaecht $K$, Van Herck $P$, et al. Effects of clinical pathways in the joint replacement: a meta-analysis. BMC Med 2009;7:32.

15. Arsalani-Zadeh R, ElFadl D, Yassin N, MacFie J. Evidence-based review of enhancing postoperative recovery after breast surgery. $\mathrm{Br} J$ Surg 2011;98:181-196.

16. Brustia P, Renghi A, Gramaglia L, et al. Mininvasive abdominal aortic surgery. Early recovery and reduced hospitalization after multidisciplinary approach. J Cardiovasc Surg 2003;44:629-635.

17. Podore PC, Throop EB. Infrarenal aortic surgery with a 3-day hospital stay: A report on success with a clinical pathway. J Vasc Surg 1999;29:787-792.

18. Wasowicz-Kemps DK, Bliemer B, Boom FA, de Zwaan NM, van Ramshorst B. Laparoscopic gastric banding for morbid obesity: outpatient procedure versus overnight stay. Surg Endosc 2006;20: 1233-1237.

19. McCarty TM, Arnold DT, Lamont JP, Fisher TL, Kuhn JA. Optimizing outcomes in bariatric surgery: outpatient laparoscopic gastric bypass. Ann Surg 2005;242:494-498; discussion 498-501.

20. Kirsh EJ, Worwag EM, Sinner M, Chodak GW. Using outcome data and patient satisfaction surveys to develop policies regarding minimum length of hospitalization after radical prostatectomy. Urology 2000;56:101-106; discussion 106-107.

21. Lassen K, Coolsen MM, Slim K, et al. Guidelines for perioperative care for pancreaticoduodenectomy: Enhanced Recovery After Surgery (ERAS(R)) Society recommendations. World J Surg 2013;37:240-258.

22. Lassen K, Coolsen MM, Slim K, et al. Guidelines for perioperative care for pancreaticoduodenectomy: Enhanced Recovery After Surgery (ERAS(R)) Society recommendations. Clin Nutr 2012;31:817-830.

23. Coolsen MM, Clermonts SH, van Dam RM, et al. Development of a composite endpoint for randomized controlled trials in pancreaticoduodenectomy. World J Surg 2014;38:1468-1475. 
24. Maessen JM, Dejong CH, Kessels AG, von Meyenfeldt MF, Enhanced Recovery After Surgery G. Length of stay: an inappropriate readout of the success of enhanced recovery programs. World J Surg 2008;32:971-975.

25. Fiore JF, Jr., Faragher IG, Bialocerkowski A, Browning L, Denehy L. Time to readiness for discharge is a valid and reliable measure of short-term recovery after colorectal surgery. World J Surg 2013;37: 2927-2934.

26. Stoot JH, van Dam RM, Busch OR, et al. The effect of a multimodal fast-track programme on outcomes in laparoscopic liver surgery: a multicentre pilot study. HPB (Oxford) 2009;11:140-144.

27. Maessen JM, Hoff C, Jottard K, et al. To eat or not to eat: facilitating early oral intake after elective colonic surgery in the Netherlands. Clin Nutr 2009;28:29-33.

28. Gillissen F, Hoff C, Maessen JM, et al. Structured synchronous implementation of an enhanced recovery program in elective colonic surgery in 33 hospitals in The Netherlands. World J Surg 2013;37: 1082-1093. 
Appendices 


\title{
APPENDIX 1: WEB SURVEY PART I AND II
}

\section{Introduction} \\ This survey consists of four parts: \\ PART I \\ A systematic search was performed using PubMed, Embase and the Cochrane Library in order to \\ identify clinical outcomes that have been used as primary or secondary endpoint in randomized \\ controlled trials specific for pancreaticoduodenectomy (PD) published in the last 6 years. \\ 17 specific complications for pancreaticoduodenectomy were selected. \\ You are asked to select which of the listed complications should be included as component of a PD \\ specific composite endpoint (CEP).

\section{PART II} \\ You are asked to tell us which other complications you would include in the PD specific CEP.

\section{PART III} \\ Which problems do you foresee and should be anticipated on, using this CEP in PD trials?

\section{PART IV} \\ Are you interested in using the Accordion severity grading system for surgical complications by \\ Strasberg et al. (derived from the Dindo-Clavien classification) in future PD trials?
}

\section{Background composite endpoint components selection.}

We ask you to select complications that in your opinion should be included as components of the PD specific composite endpoint (CEP).

For your information, we provide some general characteristics of CEPs $\left({ }^{*}\right)$.

1. A CEP is a combined parameter that captures the number of patients experiencing one or more specified complications within the CEP

2. The components of a CEP should be ascertainable without bias and well defined

3. The components of the CEP must be associated with the primary objective of a clinical trial (e.g. the effect of an intervention on surgery related mortality and morbidity)

4. The validity of a CEP depends on similarity in

- clinical importance of the components from the patient's perspective (preferably: equal or nearly equal severity grade)

- effect of the intervention on the incidence of the component (preferably: equal or nearly equal effect size)

- incidence rate of each component (preferably: equal or nearly equal incidence rate)

(*) Montori VM et al. Validity of composite end points in clinical trials. BMJ 2005;330(7491):594-596 


\section{Part I. Which complications should be included in the PD specific composite}

Proposed components are listed in the first column.

Incidence range and amount of times that endpoint was reported as outcome in a total of 33 RCT's.

Select which complications should be included in the PD specific composite endpoint according to you.

\begin{tabular}{|c|c|c|}
\hline & YES & NO \\
\hline $\begin{array}{l}\text { Pleural effusion. } \\
\text { median incidence:0\%-52.6\%. } \\
\text { Reported as outcome: } 21.2 \% \text {. }\end{array}$ & $\square$ & $\square$ \\
\hline $\begin{array}{l}\text { Ascites. } \\
\text { median incidence:0.9\%-16\%. } \\
\text { Reported as outcome: } 15.1 \% \text {. }\end{array}$ & $\square$ & $\square$ \\
\hline $\begin{array}{l}\text { Wound infection. } \\
\text { median incidence:0\%-46\%. } \\
\text { Reported as outcome:60.6\% }\end{array}$ & $\square$ & $\square$ \\
\hline $\begin{array}{l}\text { Intra-abdominal abscess. } \\
\text { median incidence:0\%-35.2\%. } \\
\text { Reported as outcome: } 57.5 \% \text {. }\end{array}$ & $\square$ & $\square$ \\
\hline $\begin{array}{l}\text { Bile } \\
\text { leakage/hepatojejunostomy leakage. } \\
\text { median incidence:0\%-8\%. } \\
\text { Reported as outcome:57.5\%. }\end{array}$ & $\square$ & $\square$ \\
\hline $\begin{array}{l}\text { Gastrojejunostomy leakage. } \\
\text { median incidence: } 0-2 \% \text {. } \\
\text { Reported as outcome: } 33.3 \% \text {. }\end{array}$ & $\square$ & $\square$ \\
\hline $\begin{array}{l}\text { Sepsis. } \\
\text { median incidence:0\%-21\%. } \\
\text { Reported as outcome:21.2\%. }\end{array}$ & $\square$ & $\square$ \\
\hline $\begin{array}{l}\text { Pneumonia. } \\
\text { median incidence:0.9\%-52.6\%. } \\
\text { Reported as outcome:36.3\%. }\end{array}$ & $\square$ & $\square$ \\
\hline $\begin{array}{l}\text { Intra-abdominal hemorrhage/ post- } \\
\text { pancreaticoduodenectomy hemorrhage. } \\
\text { median incidence:0\%-11.1\%. } \\
\text { Reported as outcome: } 57.5 \% \text {. }\end{array}$ & $\square$ & $\square$ \\
\hline $\begin{array}{l}\text { Pancreatic fistula. } \\
\text { median incidence:0\%-42\%. } \\
\text { Reported as outcome: } 75.7 \% \text {. } \\
\text { Pancreaticojejunostomy leakage. } \\
\text { median incidence:0\%- } 21 \% \text {. } \\
\text { Reported as outcome: } 84.8 \% \text {. }\end{array}$ & $\square$ & $\square$ \\
\hline $\begin{array}{l}\text { Delayed Gastric Emptying. } \\
\text { median incidence:0\%-58\%. } \\
\text { Reported as outcome: } 75.5 \% \text {. }\end{array}$ & $\square$ & $\square$ \\
\hline $\begin{array}{l}\text { Diabetis Mellitus de novo. } \\
\text { median incidence:-. } \\
\text { Reported as outcome: } 21.1 \% \text {. }\end{array}$ & $\square$ & $\square$ \\
\hline
\end{tabular}




\begin{tabular}{|lcc|}
\hline $\begin{array}{l}\text { Acute renal failure. } \\
\text { median incidence:0\%-3.7\%. }\end{array}$ & $\square$ \\
Reported as outcome:0.9\%. & $\square$ \\
\hline $\begin{array}{l}\text { Requirement of blood transfusion. } \\
\text { median incidence:1.5\%-17\%. } \\
\text { Reported as outcome:21.2\%. }\end{array}$ & $\square$ \\
\hline $\begin{array}{l}\text { Operative mortality. } \\
\text { median incidence:0\%-8.3\%. } \\
\text { Reported as outcome:69.6\%. }\end{array}$ & $\square$ \\
\hline $\begin{array}{l}\text { Costs. } \\
\text { median incidence:2700-6400 euro. } \\
\text { Reported as outcome:3\%. }\end{array}$ \\
\hline $\begin{array}{l}\text { Quality of life. } \\
\text { median incidence:-. } \\
\text { Reported as outcome:12.1\%. }\end{array}$ \\
\hline
\end{tabular}




\section{PART II. Comment on the components of the CEP.}

We would like to ask you if there is a potential component that isn't named in PART I

and should be included in a PD specific composite endpoint according to you.

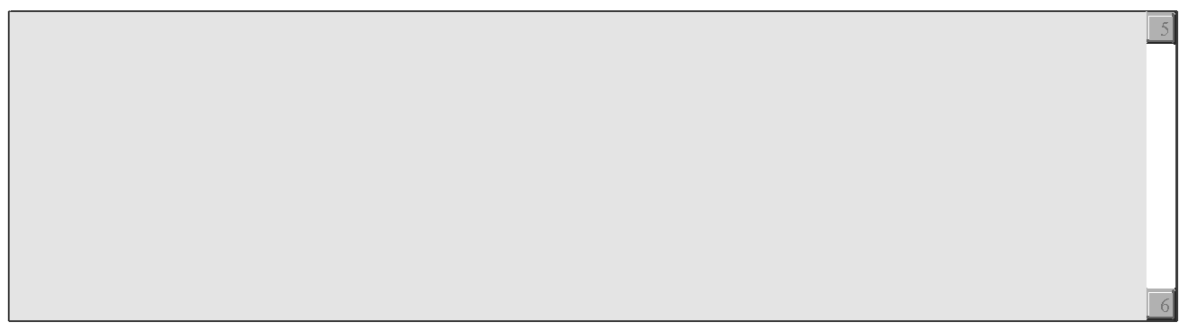

Would you be willing to share your data from a previously conducted randomized controlled trial in pancreatic surgery for determination of the current incidence rate and validation of the PD specific composite endpoint?

\section{Part III. Problems that may occur.}

Do you foresee problems that should be anticipated on, using this CEP in PD trails?

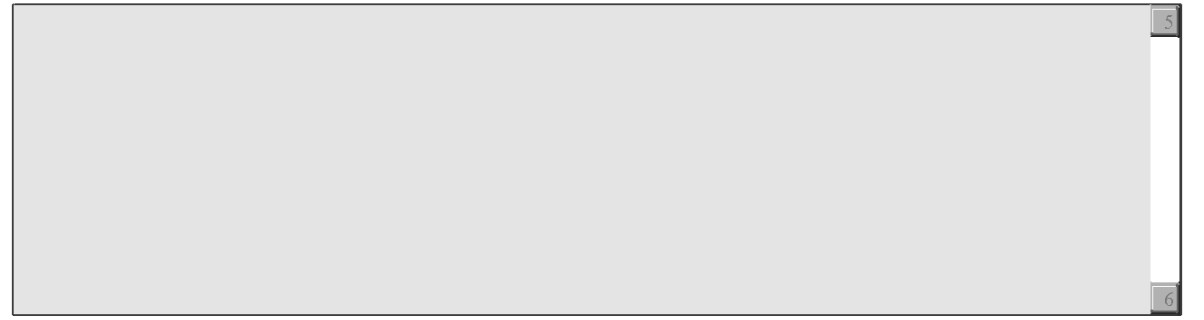




\section{Part IV. The Accordion severity grading system for surgical complications}

\section{Accordion Classification(*)}

The Accordion classification was developed as a simplified version of the Dindo-Clavien Classification. It appears the Grades I-V of the Dindo-Clavien classification leave some discussion. Furthermore, researchers tend to contract the different grades (e.g. grade $1 / 2$ and $3 a$ to grade I, and grade $3,4 a / b$ to grade II) and in many trials grades are labeled with quantitative measures (mild, moderate, severe). This means results from different trials are still not well comparable. The Accordion classification is more transparent and above all, Dindo-Clavien grades can be easily calculated from this classification when needed, while the other way around this is not possible. Below, you will find the Accordion Classification.(Dindo-Clavien grades between breaks.)

\section{Grade Mild}

1.(1.) Requires only minor invasive procedures that can be done at the bedside, such as insertion of intravenous lines, urinary catheters, and nasogastric tubes, and drainage of wound infections. Physiotherapy and anti-emetics, antipyretics, analgesics, diuretics, electrolytes, and physiotherapy are permitted.

\section{Grade Moderate}

2.(2.) Requires pharmacologic treatment with drugs other than such allowed for minor complications, eg, antibiotics. Blood transfusions and total parenteral nutrition are also included.

\section{Grade Severe}

3.(3.) No general anesthesia: requires management by an endoscopic, interventional procedure or reoperation without general anesthesia.

4.(3b) General anesthesia or single-organ failure.

5.(4a-4b) General anesthesia and single organ failure or multisystem organ failure ( 2 organ systems).

\section{Grade Death}

6.(5) Postoperative death.

Are you interested in using the Accordion severity grading system for surgical complications by Strasberg et al. instead of Dindo-Clavien severity grading scale in future PD trials?

\section{$\left({ }^{*}\right)$ Literat}

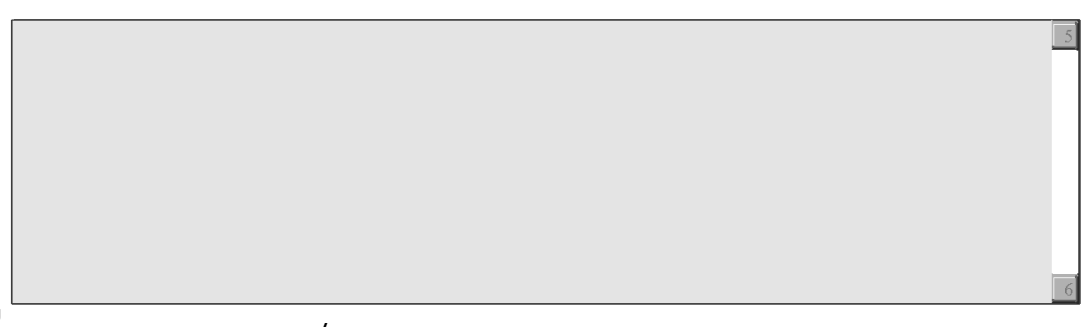

- Strasberg SM et al. The Accordion severity grading system of surgical complications. Ann Surg. 2009;250:177-86.

- Strasberg SM et al. Quantitative weighting of postoperative complications based on the accordion severity grading system: demonstration of potential impact using the American College of Surgeons national surgical quality improvement program. J Am Coll Surg. 2010;210:286-98.

\section{End of this survey.}

On behalf of the CEP Pancreas Team we would like to thank you for your time and your appreciated opinion. 


\section{Composite endpoint components and definitions}

After the first part of the worldwide survey under pancreatic surgeons for developing a Composite Endpoint for

Pancreatic Surgery, the following components were identified to be included in the endpoint.

- Intra-abdominal abscess

- Bile leakage

- Gastrojejunostomy leakage

- Leakage of the pancreatic anastomosis/pancreatic fistula

- Sepsis

- Post pancreaticoduodenectomy hemorrhage

- Delayed gastric emptying

- Mortality

The individual components of the CEP are defined as follows:

\section{Intra-abdominal abscess ${ }^{1}$}

Any quantity of purulent fluid leaking via the abdominal drain; walled-off collection of pus in the abdominal cavity at the time of radiological imaging, reoperation or percutaneous drainage; fluid in drain or intra-abdominal collection should

be culture-positive.

Bile leakage/hepaticojejunostomy leakage ${ }^{1}$

Any quantity of bile leaking via the abdominal wound or drains at least $48 \mathrm{~h}$ after surgery; intra-abdominal collection of bile at the time of radiological imaging, reoperation or percutaneous drainage; cholangiographic evidence of contrast leakage. Fluid in drain or intra-abdominal collection should have a bilirubin content at least three times higher than the upper normal serum level in patients with normal serum bilirubin levels after surgery, or a 50 per cent higher serum bilirubin level than that in patients with raised serum bilirubin levels after surgery.

\section{Sepsis}

Definition from the 2001 International Sepsis Definitions Conference ${ }^{2}$

Sepsis is the clinical syndrome defined by the presence of both infection and a systemic inflammatory response.

\section{Infection}

Pathological process caused by invasion of normally sterile tissue or fluid or body cavity by pathogenic or potentially pathogenic microorganisms. Systemic inflammatory response syndrome Body temperature higher than $38^{\circ} \mathrm{C}$ or lower than $36^{\circ} \mathrm{C}$; heart rate higher than 90 beats/min; hyperventilation evidenced by respiratory rate higher than 20 per min or arterial partial pressure of carbon dioxide lower than $32 \mathrm{mmHg}$; white blood cell count higher than 12000 cells/ $\mu$ l or lower than 4000 cells $/ \mu l$.

\section{Post pancreaticoduodenectomy hemorrhage}

According to the ISGPS classification of post pancreaticoduodenectomy hemorrhage ${ }^{3}$ :

The three different grades of PPH (grades A, B, and C) are defined according to the time of onset, site of bleeding, severity, and clinical impact.(thus all these items should be reported)

A. Mild, early postoperative bleeding without major clinical impact.

B. Severe, early postoperative bleeding with a consecutive change in a given clinical pathway.

C. Severe, late postoperative bleeding with immediate diagnostic and therapeutic consequences Operative mortality. Death of a patient during or after the initial surgical procedure that is related to the surgical procedure.

\section{Operative mortality}

Death of a patient, during or within 90 days after the initial surgical procedure, that is related to the surgical procedure. 


\section{Definition of the components II}

Pancreatic fistula/leakage of the pancreatic anastomosis

Defined according to the International Study Group of Pancreatic Fistula ${ }^{4}$

A pancreatic fistula is an abnormal communication between the pancreatic ductal epithelium and another epithelial surface containing pancreasderived, enzyme-rich fluid. However, a Post Operative Pancreatic Fistula represents failure of healing/sealing of a pancreatic- enteric anastomosis, or it may represent a parenchymal leak not directly related to an anastomosis such as one originating from the raw pancreatic surface (eg, left or central

pancreatectomy, enucleation, and/or trauma). In this case, there is a leak from the pancreatic ductal system into and around the pancreas and not necessarily to another epithelialized surface (eg, via a surgical drain).

\section{Grading}

\section{Grade A:}

This grade has no clinical impact, requires little change in management or deviation from the normal clinical pathway. The patient is fed orally and remains clinically well, the use of total parenteral nutrition, antibiotics, or somatostatin analogues are not indicated. A computed tomographic (CT) scan typically shows no peripancreatic fluid collections. Grade A POPF is not associated with a delay in hospital discharge and is managed frequently by slow removal of the operatively placed drains.

\section{Grade B:}

This grade requires a change in management or adjustment in the clinical pathway. Often the patient is kept with nothing by mouth (NPO) and is supported with partial or total parenteral or enteral nutrition. The peripancreatic drains are usually maintained in place, but if the drains are not functioning to fully drain the fistula, a CT scan may show peripancreatic collection(s) requiring repositioning of the drains. When associated with abdominal pain, fever, and/or leukocytosis, antibiotics are usually required; somatostatin analogues may also be used. Grade B pancreatic fistula usually leads to a delay in discharge, or readmission. If an invasive procedure is needed, the POPF shifts into grade $\mathrm{C}$.

\section{Grade C:}

In a grade $\mathrm{C}$, a major change in clinical management or deviation from the normal clinical pathway occurs. Clinical stability may be borderline. Clinical intervention is aggressive with the patient kept NPO and Total parenteral nutrition or enteral nutrition, intravenous antibiotics, and somatostatin analogues instituted, often in an intensive care unit setting. A CT scan usually shows worrisome, peripancreatic fluid collection(s) that require percutaneous drainage. The patient typically requires an extended hospital stay with a major delay in hospital discharge.

\section{Delayed Gastric Emptying (DGE)}

Defined according to the International Study Group of Pancreatic Surgery ${ }^{5}$

Consensus definition of DGE after pancreatic surgery.

\section{DGE grade A:}

These patients require a nasogastrictube (NGT) for about 4-7 days after surgery or reinsertion of the tube on or after postoperative day (POD) 3. The patients are unable to tolerate solid oral intake by POD 7 . Vomiting/gastric distension doesn't necessarily occur, the use of prokinetics is usually not necessary.

\section{DGE grade B:}

Patients with DGE grade $B$ require NGT for about 8-14 days after surgery or reinsertion of the tube on POD 7. Vomiting/gastric distension always occurs in a grade B DGE patient. The use of prokinetics is a necessity in grade B DGE.

\section{DGE grade C:}

Patients with DGE grade $c$ require NGT for more than 14 days after surgery or reinsertion of the tube on POD 14. Vomiting/gastric distension always occurs in a grade C DGE patient. The use of prokinetics is a necessity in grade C DGE. 


\section{Definition of the components III}

\section{Gastrojejunostomy leakage ${ }^{6}$}

Anastomotic incompetence documented either by confirmatory upper gastrointestinal contrast x-rays, CT scans, or reoperation.

\section{References}

1. van den Broek MA. et al. Development of a composite endpoint for randomized controlled trials in liver surgery. Br J Surg. 2011;98:1138-45.

2. Levy $M$ et al. 2001 SCCM/ESICM/ACCP/ATS/SIS International Sepsis Definitions Conference. Intensive Care Med. 2003;29:530-8

3. Welsch T, Eisele H, Zschäbitz S, Hinz U, Büchler MW, Wente MN. Critical appraisal of the International Study Group of Pancreatic Surgery (ISGPS) consensus definition of postoperative hemorrhage after pancreatoduodenectomy. Langenbecks Arch Surg.2011;396:783-91. Epub 2011 May 25

4. Claudio Bassi et al. Postoperative pancreatic fistula:An international study group (ISGPF) definition. 2005 J Surgery volume 138, Number 1

5. Wente $\mathrm{M}$ et al. Delayed gastric emptying (DGE) after pancreatic surgery: A suggested definition by the International Study Group of Pancreatic Surgery (ISGPS) J Surgery . 2007;142:761-8.

6. James W. Maher et al. Drain Amylase Levels Are an Adjunct inDetection of Gastrojejunostomy Leaks after Roux-en-Y Gastric BypassJ Am Coll Surg 2009, 208:881-886.

Do you agree with including the aforementioned items in the pancreas surgery specific CEP?

YES

NO

If no what should be added? 


\title{
APPENDIX 2 DEFINITIONS OF COMPLICATIONS AFTER PANCREATICODUODENECTOMY
}

\author{
Intra-abdominal abscess \\ Any quantity of purulent fluid leaking via the abdominal drain; walled-off collection of pus in the \\ abdominal cavity at the time of radiological imaging, reoperation or percutaneous drainage; fluid in drain \\ or intra-abdominal collection should be culture-positive.

\section{Sepsis} \\ Definition from the 2001 International Sepsis Definitions Conference ${ }^{1}$ \\ Sepsis is the clinical syndrome defined by the presence of both infection and a systemic inflammatory \\ response. \\ Infection \\ Pathological process caused by invasion of normally sterile tissue or fluid or body cavity by pathogenic or \\ potentially pathogenic microorganisms.

\section{Systemic inflammatory response syndrome} \\ Body temperature higher than $38^{\circ} \mathrm{C}$ or lower than $36^{\circ} \mathrm{C}$; heart rate higher than 90 beats $/ \mathrm{min}$; \\ hyperventilation evidenced by respiratory rate higher than 20 per min or arterial partial pressure of \\ carbon dioxide lower than $32 \mathrm{mmHg}$; white blood cell count higher than $12000 \mathrm{cells} / \mu \mathrm{l}$ or lower than \\ 4000 cells/ $\mu$ l.
}

\section{Gastrojejunostomy leakage}

Anastomotic incompetence documented either by confirmatory upper gastrointestinal contrast, x-rays, CT-scans or reoperation.

\section{Post pancreatic surgery hemorrhage}

Any quantity of blood leaking via the abdominal drain either with or without a drop in hemoglobin; intraabdominal active bleeding during radiological imaging or reoperation. Other sources of hemorrhage should be excluded.

According to the ISGPS classification of post pancreaticoduodenectomy hemorrhage:

a) Mild, early postoperative bleeding without major clinical impact

b) Severe, early postoperative bleeding with a consecutive change in a given clinical pathway

c) Severe, late postoperative bleeding with immediate diagnostic and therapeutic consequences

\section{Delayed Gastric Emptying (DGE)}

Defined according to the International Study Group of Pancreatic Surgery ${ }^{2}$

a) These patients require a nasogastrictube (NGT) for about 4-7 days after surgery or reinsertion of the tube on or after postoperative day (POD) 3. The patients are unable to tolerate solid oral intake by POD 7. Vomiting/gastric distension doesn't necessarily occure, the use of prokinetics is usually not necessary.

b) Patients with DGE grade B require NGT for about 8-14 days after surgery or reinsertion of the tube on POD 7. Vomiting/gastric distension always occures in a grade B DGE patient. The use of prokinetics is a necessity in grade B DGE.

c) Patients with DGE grade c require NGT for more than 14 days after surgery or reinsertion of the tube on POD 14. Vomiting/gastric distension always occures in a grade C DGE patient. The use of prokinetics is a necessity in grade C DGE. 


\section{Bile leakage/hepaticojejeunostomy leakage}

Any quantity of bile leaking via the abdominal wound or drains at least $48 \mathrm{~h}$ after surgery; intra-abdominal collection of bile at the time of radiological imaging, reoperation or percutaneous drainage; cholangiographic evidence of contrast leakage. Fluid in drain or intra-abdominal collection should have a bilirubin content at least three times higher than the upper normal serum level in patients with normal serum bilirubin levels after surgery, or a 50 per cent higher serum bilirubin level than that in patients with raised serum bilirubin levels after surgery.

\section{Pancreatic fistula/Pancreaticojejunostomy leakage}

Defined according to the International Study Group of Pancreatic Fistula ${ }^{3}$

A pancreatic fistula is an abnormal communication between the pancreatic ductal epithelium and another epithelial surface containing pancreasderived, enzyme-rich fluid. However, a Post Operative Pancreatic Fistula represents failure of healing/sealing of a pancreatic-

enteric anastomosis, or it may represent a parenchymal leak not directly related to an anastomosis such as one originating from the raw pancreatic surface (eg, left or central pancreatectomy, enucleation, and/or trauma). In this case, there is a leak from the pancreatic ductal system into and around the pancreas and not necessarily to another epithelialized surface (eg, via a surgical drain).

\section{Grading}

a) This grade has no clinical impact, requires little change in management or deviation from the normal clinical pathway. The patient is fed orally and remains clinically well, the use of total parenteral nutrition, antibiotics, or somatostatin analogues are not indicated. A computed tomographic (CT) scan typically shows no peripancreatic fluid collections. Grade A POPF is not associated with a delay in hospital discharge and is managed frequently by slow removal of the operatively placed drains.

b) This grade requires a change in management or adjustment in the clinical pathway. Often the patient is kept with nothing by mouth and is supported with partial or total parenteral or enteral nutrition. The peripancreatic drains are usually maintained in place, but if the drains are not functioning to fully drain the fistula, a CT scan may show peripancreatic collection(s) requiring repositioning of the drains. When associated with abdominal pain, fever, and/or leukocytosis, antibiotics are usually required; somatostatin analogues may also be used. Grade B pancreatic fistula usually leads to a delay in discharge, or readmission. If an invasive procedure is needed, the POPF shifts into grade $\mathrm{C}$.

c) In a grade $\mathrm{C}$, a major change in clinical management or deviation from the normal clinical pathway occurs. Clinical stability may be borderline. Clinical intervention is aggressive with the patient kept nill by mouth and (total) parenteral nutrition or enteral nutrition, intravenous antibiotics, and somatostatin analogues are instituted, often in an intensive care unit setting. A CT scan usually shows worrisome, peripancreatic fluid collection(s) that require percutaneous drainage. The patient typically requires an extended hospital stay with a major delay in hospital discharge.

\section{Operative mortality}

Death of a patient during or within 90 days after the initial surgical procedure that is related to the surgical procedure.

\section{References}

1. Levy MM, Fink MP, Marshall JC, Abraham E, Angus D, Cook D, Cohen J, Opal SM, Vincent JL, Ramsay G; International Sepsis Definitions Conference. 2001 SCCM/ESICM/ACCP/ATS/SIS International Sepsis Definitions Conference. Intensive Care Med. 2003;29:530-538.

2. Wente MN, Bassi C, Dervenis C, Fingerhut A, Gouma DJ, Izbicki JR, Neoptolemos JP, Padbury RT, Sarr MG, Traverso LW, Yeo CJ, Büchler MW. Delayed gastric emptying (DGE) after pancreatic surgery: A suggested definition by the International Study Group of Pancreatic Surgery (ISGPS) J Surgery . 2007;142:761-768.

3. Bassi C, Dervenis C, Butturini G, Fingerhut A, Yeo C, Izbicki J, Neoptolemos J, Sarr M, Traverso W, Buchler M; International Study Group on Pancreatic Fistula Definition. Postoperative pancreatic fistula:An international study group (ISGPF) definition. J Surgery 2005;138:8-13. 


\section{APPENDIX 3 FLOWCHART PANDA TRIAL}

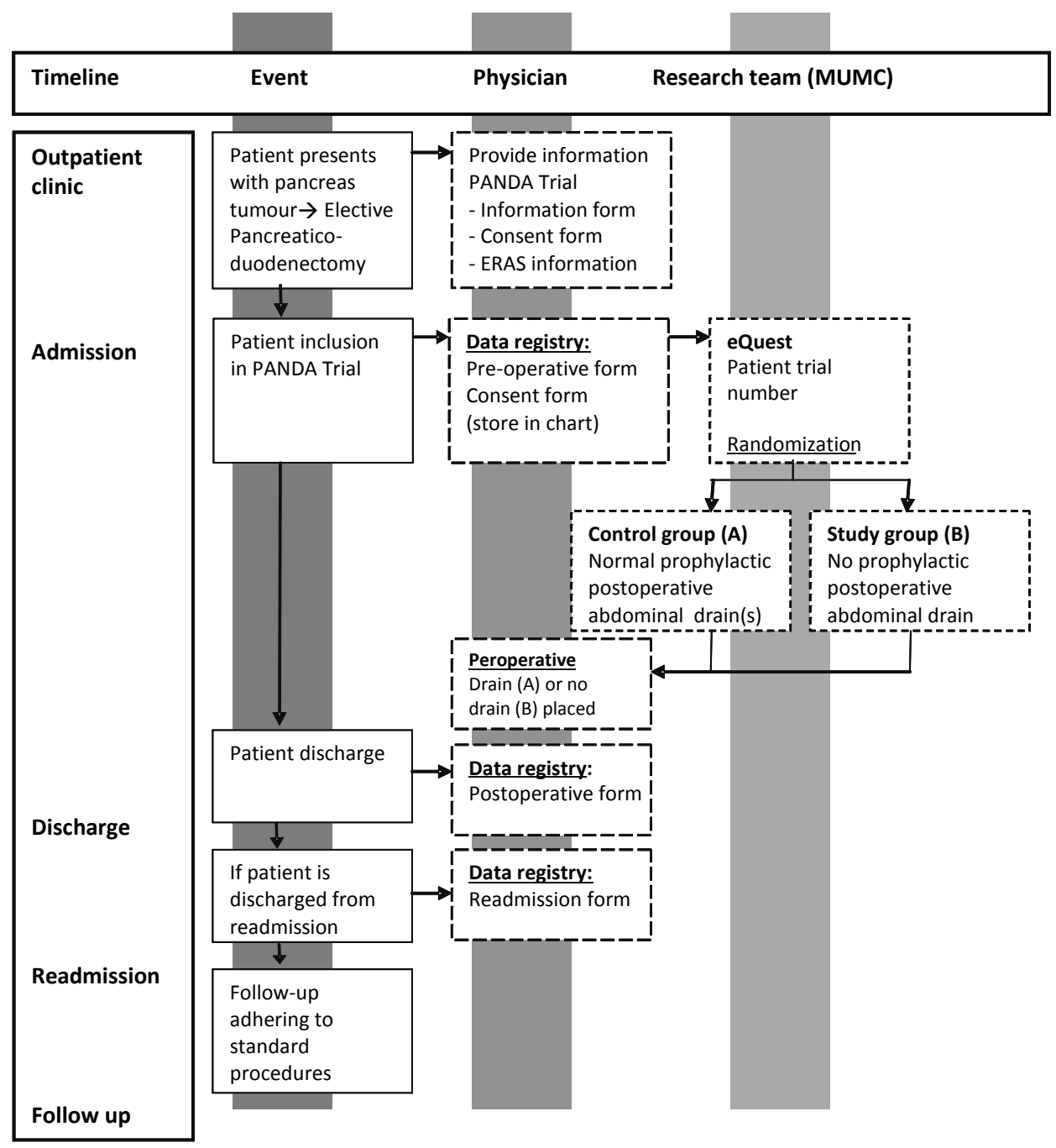




\section{APPENDIX 4: ELEMENTS OF AN ERAS PROGRAMME FOR PANCREATICODUODENECTOMY}

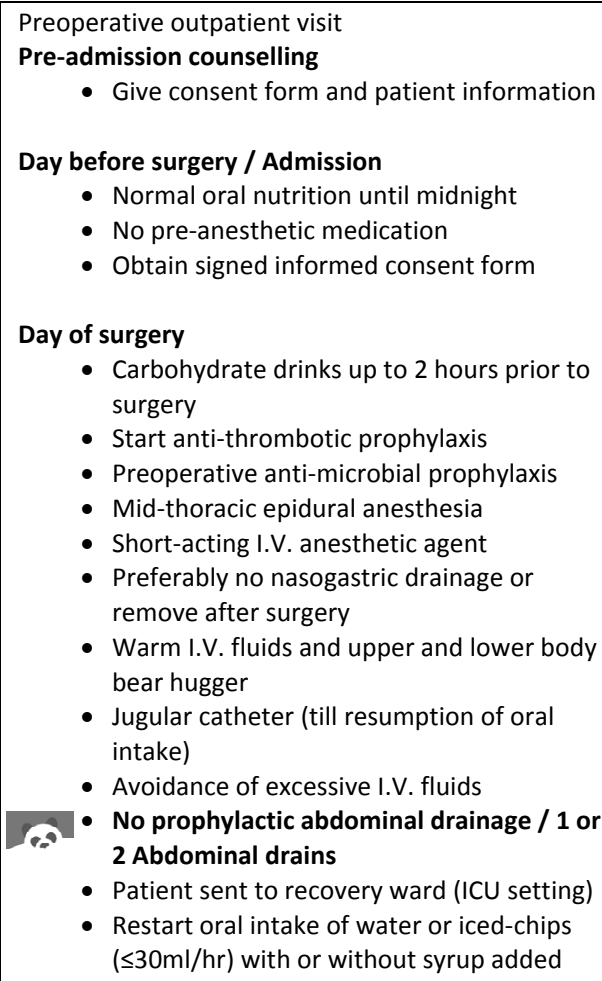

- Give consent form and patient information

Day before surgery / Admission

- Normal oral nutrition until midnight

- No pre-anesthetic medication

- Obtain signed informed consent form

Day of surgery

- Carbohydrate drinks up to 2 hours prior to surgery

- Start anti-thrombotic prophylaxis

- Preoperative anti-microbial prophylaxis

- Mid-thoracic epidural anesthesia

- Short-acting I.V. anesthetic agent

- Preferably no nasogastric drainage or remove after surgery

- Warm I.V. fluids and upper and lower body bear hugger

- Jugular catheter (till resumption of oral intake)

- Avoidance of excessive I.V. fluids

- No prophylactic abdominal drainage / 1 or 2 Abdominal drains

- Patient sent to recovery ward (ICU setting)

- Restart oral intake of water or iced-chips $(\leq 30 \mathrm{ml} / \mathrm{hr}$ ) with or without syrup added

POD 1

- Patient sent to surgical ward

- Patient mobilizes a minimum of four times a day

- Patient is capable of drinking at least 1.5 Litres of water

- Continue portable epidural analgesia

- Antiemetics if based on nausea score

- $1000 \mathrm{mg}$ Paracetamol every 6 hours

- $1000 \mathrm{mg}$ Magnesiumoxide twice daily

POD 2

- If patient is capable resumption of regular diet

- Continue mobilization

- Remove urinary catheter

- Continuation epidural analgesia

- Stop low dose opioids

- Check functional recovery criteria
POD 3

- Stop epidural analgesia

- Discontinue I.V. fluids

- Start NSAIDs based on pain score

- Continue mobilization

- Regular diet

- Check functional recovery criteria

POD 4

(8.) Prophylactic drain removal (if appropriate)

- Continue analgesics based on pain score

- Continue mobilization

- Regular diet

- Check functional recovery criteria

POD 5

- Continue analgesics based on pain score

- Continue mobilization

- Regular diet

- Check functional recovery criteria

POD 6

- Continue analgesics based on pain score

- Continue mobilization

- Regular diet

- Check functional recovery

POD 7

- Continue mobilization

- Regular diet

- Check functional recovery criteria

- Make outpatient appointment for after discharge

POD 8

- Continue analgesics based on pain score

- Continue mobilization

- Regular diet

- Check functional recovery criteria

- Discharge from hospital 


\title{
APPENDIX 5 DEFINITIONS OF COMPLICATIONS
}

\author{
Mortality \\ Death of a patient, during or within 90 days after the initial surgical procedure that is related to the surgical \\ procedure.
}

\section{Readmission}

Every re-hospitalization due to a complication of the index hospitalization within the first 6 months.

\section{Intra-abdominal abscess}

Any quantity of purulent fluid leaking via the abdominal drain; walled-off collection of pus in the abdominal cavity at the time of radiological imaging, reoperation or percutaneous drainage; fluid in drain or intraabdominal collection should be culture positive.

Wound infection defined according to the Centers for Disease Control and Prevention

Superficial incisional surgical-site infection and deep incisional surgical site infection, which occurs within 30 days after surgery. This includes both the superficial surgical-site infections and the deep incisional surgical site infections. Most of these wound infections were opened at bedside by the physician or surgeon.

\section{Pancreatic fistula/pancreaticojejunostomy leakage}

Defined according to the International Study Group of Pancreatic Fistula (Grade A, B and C)

\section{Delayed gastric emptying}

Defined according to the International Study Group of Pancreatic Surgery (Grade A,B and C)

\section{Biliodigestive anastomosis leakage}

Any quantity of bile leaking via the abdominal wound or drains at least $48 \mathrm{~h}$ after surgery, intra-abdominal collection of bile (confirmed radiologically) or cholangiographic evidence of contrast leakage. Laboratory: three times higher bilirubin in the drain or intra-abdominal collection compared with normal serum bilirubin levels.

\section{Sepsis/multi organ failure}

Definition from the 2001 International Sepsis Definitions Conference

\section{Post pancreaticoduodenectomy hemorrhage}

Definition according to the ISGPS classification of post pancreaticoduodenectomy hemorrhage

\section{Gastrojejunostomy leakage}

Leakage of the gastrojejunostomy anastomosis documented either by confirmatory upper gastrointestinal contrast x-rays. CT scans, or reoperation.

\section{CT-drainage}

Drainage of intra-abdominal fluid collections during CT by inserting a percutaneous drain during the hospitalization or readmission period.

\section{Relaparotomy}

Any complication after the initial PPPD or Whipple operation leading to a laparotomy as an intervention, during the index hospitalization or the readmission period. 


\section{APPENDIX 6 COMPLICATIONS BY TYPE OF RESECTION}

\begin{tabular}{lll}
\hline & Whipple $(\mathrm{n}=53)$ & PPPD $(\mathrm{n}=177)$ \\
\hline Intra abdominal abscess & $7(13.2)$ & $28(15.8)$ \\
DGE & $3(5.7)$ & $19(10.7)$ \\
Pancreatic fistula & $10(18.9)$ & $18(10.2)$ \\
Gastrojejunostomy leakage & $1(1.9)$ & $3(1.7)$ \\
Bile/hepaticojejunostomy leakage & $2(3.8)$ & $11(6.2)$ \\
Post-pancreatectomy hemorrhage & $4(7.5)$ & $7(4.0)$ \\
Sepsis & $2(3.8)$ & $9(5.1)$ \\
Mortality & $4(7.5)$ & $7(4.0)$ \\
Urinary tract infection & $3(5.7)$ & $6(3.4)$ \\
Ileus & $2(3.8)$ & $7(4.0)$ \\
Abdominal wound dehiscence & $2(3.8)$ & $4(2.3)$ \\
Pneumonia & $4(7.5)$ & $12(6.8)$ \\
Wound infection & $7(13.2)$ & $23(13.0)$ \\
Diabetes Mellitus de novo & 0 & $3(1.7)$ \\
Cerebrovascular accident & 0 & $2(1.1)$ \\
Gastro-intestinal ulcer & 0 & $1(0.6)$ \\
Cholangitis & $2(3.8)$ & $1(0.6)$ \\
Dumping & 0 & $1(0.6)$ \\
Patients without complications & $35(66)$ & $102(58)$ \\
\hline
\end{tabular}

Values in parentheses are percentages.

$+p<0.05$

DGE: delayed gastric emptying 


\section{APPENDIX 7: PATIENT DEMOGRAPHICS, PATHOLOGY AND OPERATIVE DATA FOR THE 66-69 AGE GROUP}

\begin{tabular}{|c|c|c|c|}
\hline & $\begin{array}{c}\leq 65 \text { years of age } \\
(n=55)\end{array}$ & $\begin{array}{c}66-69 \text { years of age } \\
(n=25)\end{array}$ & $\geq 70$ years of age $(n=55)$ \\
\hline Age median (range) & $57(45-65)$ & $68(65-69)$ & $77(70-86)$ \\
\hline \multicolumn{4}{|l|}{ Sex } \\
\hline Male/female & $35 / 20$ & $12 / 13$ & $22 / 33$ \\
\hline \multicolumn{4}{|l|}{ ASA class } \\
\hline I & $28(51 \%)$ & $8(32 \%)$ & $1(2 \%)$ \\
\hline II & $21(38 \%)$ & $13(52 \%)$ & $34(62 \%)$ \\
\hline III & $5(9 \%)$ & $4(16 \%)$ & $20(36 \%)$ \\
\hline IV & $1(2 \%)$ & 0 & $0(0 \%)$ \\
\hline \multicolumn{4}{|l|}{ Comorbidity } \\
\hline Cardiovascular & $13(24 \%)$ & $7(28 \%)$ & $25(45 \%)$ \\
\hline Pulmonary & $2(4 \%)$ & $3(12 \%)$ & $10(18 \%)$ \\
\hline Diabetes & $6(11 \%)$ & $6(24 \%)$ & $12(21.8 \%)$ \\
\hline Renal disease & $1(2 \%)$ & $2(8 \%)$ & $6(10.9 \%)$ \\
\hline BMI (range) & $24.9(18.0-36.1)$ & $24.6(18-47)$ & $24.4(18.6-36.8)$ \\
\hline \multicolumn{4}{|l|}{ Pathology } \\
\hline Pancreatic head carcinoma & $34(62 \%)$ & $12(48 \%)$ & $33(60 \%)$ \\
\hline Duodenal cancer & $2(4 \%)$ & $4(16 \%)$ & $3(5 \%)$ \\
\hline Ampullary carcinoma & $8(15 \%)$ & $5(20 \%)$ & $12(22 \%)$ \\
\hline Distal cholangiocarcinoma & $5(9 \%)$ & $3(12 \%)$ & $5(9 \%)$ \\
\hline $\begin{array}{l}\text { Metastasis in pancreas } \\
\text { head }\end{array}$ & $1(1,8 \%)$ & $1(4 \%)$ & $0(0 \%)$ \\
\hline Chronic pancreatitis & $2(4 \%)$ & $1(4 \%)$ & $2(4 \%)$ \\
\hline IPMN & $1(2 \%)$ & 0 & $0(0 \%)$ \\
\hline Neuro-endocrine tumor & $2(4 \%)$ & 0 & $0(0 \%)$ \\
\hline \multicolumn{4}{|l|}{ Operation type } \\
\hline Whipple & $9(16 \%)$ & $3(12 \%)$ & $8(15 \%)$ \\
\hline PPPD & $46(84 \%)$ & $22(88 \%)$ & $47(85 \%)$ \\
\hline Duration of OR time ( $\mathrm{min}$ ) & $345(180-810)$ & $310(190-610)$ & 340 (217-715) \\
\hline
\end{tabular}

ASA=American Society of Anesthesiologists, BMI=Body Mass Index. PPPD=pylorus preserving pancreaticoduodenectomy 


\section{APPENDIX 8: POSTOPERATIVE OUTCOMES FOR THE 66-69 AGE GROUP}

\begin{tabular}{lccc}
\hline & $\begin{array}{c}\leq 65 \text { years of age } \\
(\mathrm{n}=55)\end{array}$ & $\begin{array}{c}66-69 \text { years of age } \\
(\mathrm{n}=25)\end{array}$ & $\begin{array}{c}\geq 70 \text { years of age } \\
(\mathrm{n}=55)\end{array}$ \\
\hline Dindo-Clavien classification & & & \\
$\quad$ Minor (I/II) & $15(27.2 \%)$ & $8(32 \%)$ & $20(36.3 \%)$ \\
$\quad$ Moderate (IIIa/b) & $15(27.2 \%)$ & $4(16 \%)$ & $8(14.5 \%)$ \\
$\quad$ Severe (Iva en IVb) & $0(0 \%)$ & $1(4 \%)$ & $2(3.6 \%)$ \\
Mortality <90days & $3(5,5 \%)$ & $1(4 \%)$ & $6(10.9 \%)$ \\
Readmissions <6 months & $11(20 \%)$ & $1(4 \%)$ & $7(12.7 \%)$ \\
Median length of hospital stay (range) & $14(6-100)$ & $14(6-64)$ & $14(6-65)$ \\
Length of stay uncomplicated (median) & $9(6-15)$ & $9(6-14)$ & $9(6-22)$ \\
Median LOS Dindo-Clavien & $10(6-100)$ & $14(6-60)$ & $14(7-59)$ \\
grade <III (range) & & & \\
Median LOS Dindo-Clavien & $32(11-83)$ & $51(26-64)$ & $33(14-65)$ \\
grade >III (range) & & & \\
Interventions & $10(18.1 \%)$ & $3(12 \%)$ & $8(14.5 \%)$ \\
$\quad$ CT/US-drainage & $6(10.9 \%)$ & $1(4 \%)$ & $4(7.2 \%)$ \\
Re-operation & &
\end{tabular}




\section{APPENDIX 9: ERAS OUTCOMES FOR THE 66-69 AGE GROUP}

\begin{tabular}{lccc}
\hline & $\begin{array}{c}\leq 65 \text { years of age } \\
(\mathrm{n}=55)\end{array}$ & $\begin{array}{c}66-69 \text { years of age } \\
(\mathrm{n}=25)\end{array}$ & $\begin{array}{c}\geq 70 \text { years of age } \\
(\mathrm{n}=55)\end{array}$ \\
\hline Resumption of fluid intake POD 1 & $53(96 \%)$ & $25(100 \%)$ & $52(95 \%)$ \\
Resumption of solid intake POD 2 & $31(56 \%)$ & $17(68 \%)$ & $35(63 \%)$ \\
Mobilization from POD 1 & $33(60 \%)$ & $17(68 \%)$ & $35(63 \%)$ \\
Drain removal on POD 4 & $26(47 \%)$ & $16(64 \%)$ & $28(51 \%)$ \\
Urinary catheter removal on POD 2 & $38(69 \%)$ & $13(52 \%)$ & $33(60 \%)$ \\
Removal epidural on POD 3 & $44(80 \%)$ & $20(80 \%)$ & $43(78 \%)$ \\
\hline
\end{tabular}




\section{APPENDIX 10}

Questionnaire for patients, part II: Targets for recovery

Please indicate how important you feel that each of the following targets is for recovery after an abdominal operation.

\begin{tabular}{|c|c|c|c|c|c|c|c|c|c|c|c|}
\hline & $\begin{array}{c}\text { NOT } \\
\text { IMPORTANT }\end{array}$ & & & & & & & & & & $\begin{array}{c}\text { VERY } \\
\text { IMPORTANT }\end{array}$ \\
\hline $\begin{array}{l}\text { 1. To be completely } \\
\text { free of pain at } \\
\text { rest }\end{array}$ & 0 & 0 & 0 & 0 & $O$ & 0 & 0 & 0 & 0 & 0 & 0 \\
\hline $\begin{array}{l}\text { 2. To be completely } \\
\text { free of pain upon } \\
\text { movement }\end{array}$ & 0 & 0 & 0 & 0 & 0 & 0 & 0 & 0 & $O$ & 0 & 0 \\
\hline $\begin{array}{l}\text { 4. To be able to eat } \\
\text { and drink as soon } \\
\text { as possible }\end{array}$ & $\mathrm{O}$ & 0 & O & 0 & $O$ & 0 & 0 & 0 & $O$ & 0 & 0 \\
\hline $\begin{array}{l}\text { 5. To be able to move } \\
\text { the bowels as soon } \\
\text { as possible }\end{array}$ & 0 & 0 & 0 & 0 & 0 & 0 & 0 & 0 & 0 & 0 & 0 \\
\hline $\begin{array}{l}\text { 7. To be discharged } \\
\text { from hospital as } \\
\text { soon as possible }\end{array}$ & 0 & 0 & 0 & 0 & 0 & 0 & 0 & 0 & 0 & 0 & 0 \\
\hline $\begin{array}{l}\text { 8. To be able to return } \\
\text { to all daily activities } \\
\text { as soon as possible }\end{array}$ & 0 & 0 & 0 & 0 & $O$ & 0 & 0 & 0 & $O$ & 0 & O \\
\hline
\end{tabular}




\section{Questionnaire for patients part III: Achieving recovery}

Think about yourself recovering successfully after an abdominal operation. How important do you view the following care items to be in achieving this recovery?

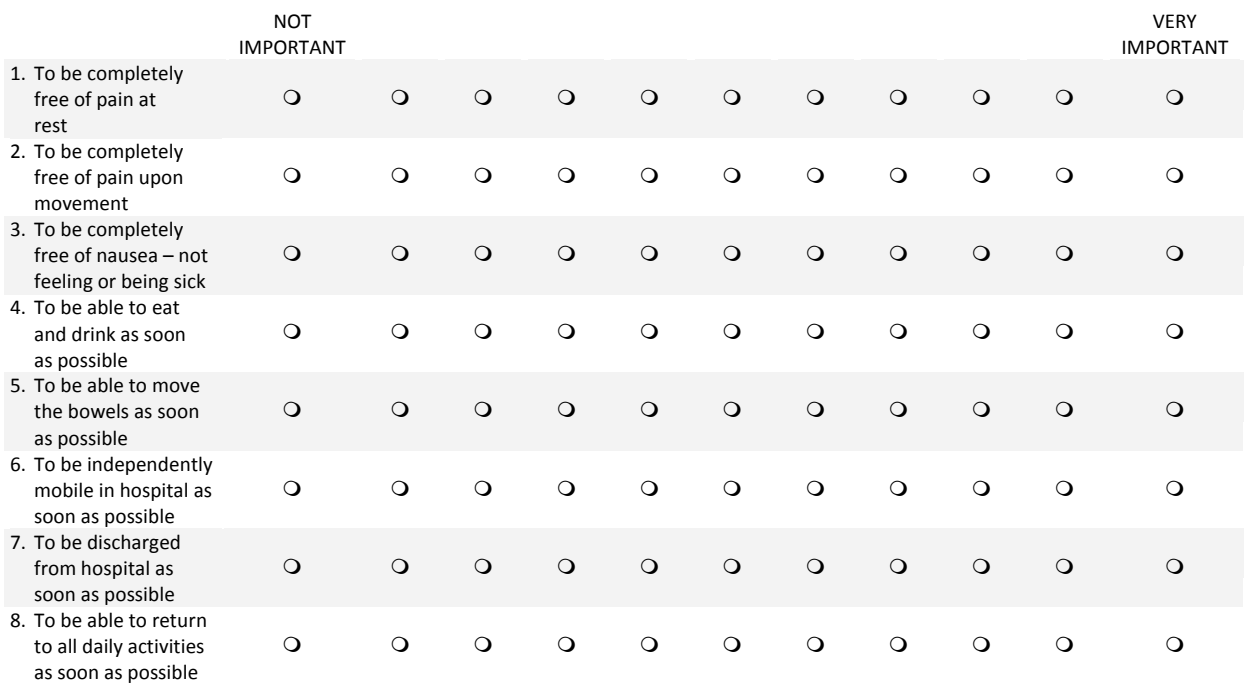




\section{APPENDIX 11}

1. Please indicate how important you feel that each of the following targets is for recovery after an abdominal operation.

\begin{tabular}{|c|c|c|c|c|c|c|c|c|c|c|c|}
\hline & $\begin{array}{c}\text { NOT } \\
\text { IMPORTANT }\end{array}$ & & & & & & & & & & $\begin{array}{c}\text { VERY } \\
\text { IMPORTANT }\end{array}$ \\
\hline $\begin{array}{l}\text { 2. To be without any } \\
\text { intra venous line as } \\
\text { soon as possible }\end{array}$ & 0 & 0 & 0 & $O$ & $O$ & 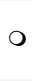 & 0 & O & $\mathrm{O}$ & 0 & $O$ \\
\hline $\begin{array}{l}\text { 4. To be allowed } \\
\text { normaldiet at will } \\
\text { after the opeation }\end{array}$ & 0 & 0 & 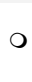 & $\mathrm{O}$ & 0 & 0 & O & $\mathrm{O}$ & 0 & O & 0 \\
\hline
\end{tabular}


2. Think about successful recovery after an abdominal operation as you perceive it. How important do you view the following care items to be in achieving this recovery?

NOT
IMPORTANT

VERY

IMPORTANT

1. Preoperative

counselling by

nurse, anesthetist

and surgeon

2. Avoidong otal

bowel preparation

preoperatively

3. Preoperative

fasting kept at

absolute minimum

4. Avoiding

preanesthetic

sedative

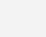

5. Oral carbohydrate loading preoperatively

6. Antimicrobial prophylaxis and skin preparation

7. Using epidural analgesia for approximately 48 hrs. postop.

8. Avoiding nasogastric tube after the operation

9. Optimising fluid

balance

10. Avoiding a wound drain

O

(2)

te

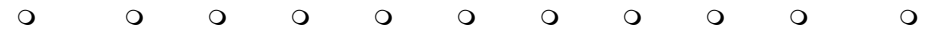

\begin{tabular}{|c|c|c|c|c|c|c|c|c|c|}
\hline 0 & 0 & 0 & 0 & 0 & 0 & 0 & 0 & $\mathrm{O}$ & 0 \\
\hline
\end{tabular}

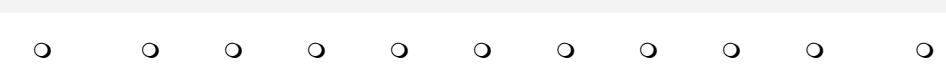

(

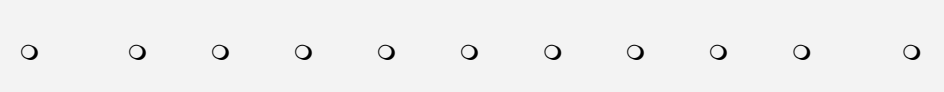

Allowing normat diet at will after the operation

12. Stimulation of gut motility

13. Promoting early and scheduled mobilisation 
List of publications 


\section{LIST OF PUBLICATIONS}

- M.M.E. Coolsen, M. Bakens, R.M. van Dam, S.W.M. Olde Damink C.H.C. Dejong. Implementing an enhanced recovery program after pancreaticoduodenectomy in elderly patients - is it feasible? World J Surg. 2014 Sep 12. Epub ahead of print

- M. Hughes, M.M.E. Coolsen, E.M. Harrison, E. Aahlin, S.J. McNally, C.H.C. De Jong, K. Lassen, S.J. Wigmore. Attitudes of patients and care providers to enhanced recovery after surgery programs following major abdominal surgery. J Surg Res. 2014 Jun 23. Epub ahead of print.

- M.M.E. Coolsen, A.Chigharoe, R.M. van Dam, L.P.S. Stassen, S.W.M. Olde Damink, C.H.C.Dejong. Improving outcome after pancreaticoduodenectomy - experiences with an enhanced recovery after surgery pathway . Dig Surg 2014;31:177-184

- M.M.E. Coolsen, S.H.E.M. Clermonts, B.Winkens, R.M. van Dam, S.W.M. Olde Damink, C.H.C. Dejong. Development of a composite endpoint for randomized controlled trials in pancreaticoduodenectomy. World J Surg 2014:38:1468-1475

- $\quad$ K.Lassen, O.Ljungqvist, C.H.C. Dejong, N.Demartines, R.W. Parks, D.N. Lobo, M.M.E. Coolsen, K.C. Fearon. Pancreaticoduodenectomy: ERAS recommendations. Clin Nutr. 2013 Oct;32(5):870-1

- M.M.E.Coolsen, R.M. van Dam, A.A. van der Wilt, K. Slim, K. Lassen, C.H.C. Dejong. Systematic review and meta-analysis of enhanced recovery after pancreatic surgery with particular emphasis on pancreaticoduodenectomies. World J Surg. 2013 Aug;37(8):1909-18

- A.A.van der Wilt, M.M.E. Coolsen, I.H. de Hingh, G.J. van der Wilt, H.Groenewoud, C.H.C. Dejong , R.M. van Dam. To drain or not to drain: a cumulative meta-analysis of the use of routine abdominal drains after pancreatic resection. HPB (Oxford). 2013 May;15(5):337-44.

- M.M.E.Coolsen, E.M. Wong-Lun-Hing, R.M. van Dam, A.A. van der Wilt, K. Slim, K. Lassen, C.H.C. Dejong. A systematic review of outcomes in patients undergoing liver surgery in an enhanced recovery after surgery pathway.. HPB (Oxford). 2013 Apr;15(4):245-51.

- K.Lassen, M.M.E. Coolsen, K. Slim, F. Carli, J.E. de Aguilar-Nascimento, M. Schäfer, R.W. Parks, K.C. Fearon, D.N. Lobo, N. Demartines, M. Braga, O. Ljungqvist, C.H.C. Dejong; ERAS ${ }^{\circledR}$ Society; European Society for Clinical Nutrition and Metabolism; International Association for Surgical Metabolism and Nutrition. Guidelines for perioperative care for pancreaticoduodenectomy: Enhanced Recovery After Surgery (ERAS ${ }^{\circledR}$ ) Society recommendations. Clin Nutr. 2012 Dec;31(6):817-30

- K.Lassen, M.M.E. Coolsen, K. Slim, F. Carli, J.E. de Aguilar-Nascimento, M. Schäfer, R.W. Parks, K.C. Fearon, D.N. Lobo, N. Demartines, M. Braga, O. Ljungqvist, C.H.C. Dejong; Enhanced Recovery After Surgery (ERAS) Society, for Perioperative Care; European Society for Clinical Nutrition and Metabolism (ESPEN); International Association for Surgical Metabolism and Nutrition (IASMEN). Guidelines for perioperative care for pancreaticoduodenectomy: Enhanced Recovery After Surgery (ERAS ${ }^{\circledR}$ ) Society recommendations. World J Surg. 2013 Feb;37(2):240-58.

- M.M.J. Caron, P.J. Emans, A. Cremers, D.A. Surtel, M.M.E. Coolsen, L.W. van Rhijn, T.J. Welting. Hypertrophic differentiation during chondrogenic differentiation of progenitor cells is stimulated by BMP-2 but suppressed by BMP-7. Osteoarthritis Cartilage. 2013 Apr;21(4):604-13

- M.M.J. Caron, P.J. Emans, M.M.E. Coolsen, L. Voss, D.A.M. Surtel, A. Cremers, L.W. van Rhijn, T.J.M.Welting. Redifferentiation of dedifferentiated human articular chondrocytes: comparison of 2D and 3D cultures. Osteoarthritis and Cartilage 2012 Oct;20(10):1170-8

- T.J.Welting, M.M.J. Caron, P.J. Emans, M.P. Janssen, K. Sanen, M.M.E. Coolsen, L. Voss, D.A. Surtel, A. Cremers, J.W. Voncken, L.W. van Rhijn. Inhibition of cyclooxygenase-2 impacts chondrocyte hypertrophic differentiation during endochondral ossification. Eur Cell Mater. 2011 Dec 19;22:420-36

- R.M. van Dam, P.O. Hendry, M.M.E. Coolsen, M.H.A. Bemelmans, K. Lassen, A.Revhaug, K.C.H. Fearon, O.J. Garden, C.H.C. Dejong. Fast track liver surgery: initial experience with a multimodal enhanced recovery programme in patients undergoing liver resection. Br J Surg 2008; 95:969-975. 
Dankwoord 


\section{DANKWOORD}

Wouw, het is af!

En wat hebben we hard gewerkt met zijn allen!

Bij deze wil ik iedereen heel hartelijk bedanken die op één of andere manier een bijdrage heeft geleverd aan dit proefschrift.

Een aantal personen zou ik graag in het bijzonder bedanken:

Prof. dr. Dejong, beste Kees: dank je wel voor alle tijd die je hebt genomen om mij een betere wetenschapper te maken, je kritische doch zeer opbouwende commentaar op de stukken die ik schreef en je vertrouwen in mij. Ik bewonder je manier van werken zowel als clinicus en als academicus. Ik hoop dat ik ooit in de buurt van jouw niveau mag komen.

Dr. Olde Damink, beste Steven: jouw gedrevenheid en wetenschappelijk succes is iets om trots en om jaloers op te zijn. Je hebt me enorm gestimuleerd om effectief en efficiënt aan mijn proefschrift te werken en dingen af te ronden op het juiste tijdstip. Het was een plezier om met je samen te werken. Dank je wel!

Dr. van Dam, beste Ronald: ooit klopte ik op jouw deur als derdejaars geneeskunde studente met de vraag of ik bij jou wetenschappelijk onderzoek kon komen doen. Ik werd hartelijk ontvangen en kon meteen beginnen om mee te werken aan een project dat ging over versnelde herstel programma's in de leverchirurgie (toen nog niet wetende dat dit later de basis van mijn proefschrift zou worden). Ik heb ontzettend veel van je geleerd in die periode en vond je manier van werken prachtig. Jouw gedachte was om jonge onderzoekers hard te laten werken maar ze als tegenprestatie te belonen door ze bijvoorbeeld mee naar MOD procedures te nemen en ze zo vroeg mogelijk al op congressen presentaties te laten houden. Dit zorgde er niet alleen voor dat het leuk was en leuk bleef maar het gaf ook een enorm groepsgevoel, een gevoel alsof je er helemaal bij hoorde dus. Dit heb ik enorm gewaardeerd en ik denk vele onderzoekers met mij. Ik wil je bedanken voor de geweldige tijd die ik hierdoor bij de HPB-groep heb gehad. Maar ik wil je met name bedanken voor alle mogelijkheden die je me hebt geboden die ervoor gezorgd hebben dat ik dit proefschrift heb kunnen schrijven.

De semi-artsen: Aart van der Wilt, Stefan Clermonts, Arwind Chigharoe, Maikel Bakens en Victor van Woerden. Jongens, grote dank voor jullie inzet en voor de prettige samenwerking, zonder jullie was het vormgeven en afronden van dit proefschrift nooit gelukt. 
De hoofdstukken in dit proefschrift, welke inmiddels allen zijn gepubliceerd of geaccepteerd in wetenschappelijk tijdschriften, zouden kwalitatief niet van dit niveau zijn geweest zonder de inbreng van alle co-auteurs. Hierbij wil ik alle co-auteurs bedanken die een bijdrage hebben geleverd aan één van de hoofdstukken.

Deze promotie heeft vanzelfsprekend niet tot stand kunnen komen zonder de leescommissie die dit proefschrift heeft beoordeeld. Ik wil dan ook de leden van de leescommissie: Prof. dr. M.F. von Meyenfeldt, Prof. dr. W.F. Buhre, Prof. dr. K.C. Conlon, Prof. dr. J.M.P. Kleijnen en Dr. I.H.J.T. de Hingh hartelijk bedanken voor hun bereidheid dit proefschrift te beoordelen.

Prof. PhD. M.F. von Meyenfeldt, Prof. PhD. W.F. Buhre, Prof. PhD. K.C. Conlon, Prof. PhD. J.M.P. Kleijnen and PhD. I.H.J.T. de Hingh, thank you very much for your willingness to join the committee to review my thesis.

Overige leden van de promotiecommissie, veel dank voor het optreden als opponent tijdens mijn verdediging

Als afsluiting wil ik graag nog enkele personen bedanken die niet direct een bijdrage hebben geleverd aan de wetenschappelijke inhoud van dit proefschrift, maar die wel heel belangrijk zijn geweest in de totstandkoming van dit proefschrift en deze promotie.

Lieve Lotte en Mechteld, lieve vriendinnetjes, ik vind het een eer dat jullie paranimf willen zijn bij mijn promotie, onwijs bedankt voor jullie steun!

Kim, David en Maikel, mijn IHPBA-matties. Wat hebben we toch een mooie tijd gehad in Seoel! De IHPBA was een heel groot congres waar we allemaal ons steentje aan bij hebben mogen dragen. Maar des te mooier nog waren de geweldige avonden en nachten in Koreaanse clubs, loungebarren en eettentjes. Dank voor de gezelligheid en de vele lachbuien!

Kim Rauwerdink, lieve schoonzus. Wat heb je mijn proefschrift toch een prachtige omslag gegeven! Ik ben er trots op dat ik zo'n creatieve schoonzus heb die telkens maar voor mij klaar staat. Je bent een schat, bedankt!

Arjen, Jon en Fen, bedankt voor alle mooie momenten in de afgelopen jaren. Ik ben heel blij dat jullie nu echt mijn familie geworden zijn!

Frans en Ivonne, mijn schoonouders. Vanaf het eerste moment dat ik bij jullie over de vloer kwam ben ik met open armen ontvangen. Dat heeft mij een enorm warm gevoel gegeven en inmiddels zijn jullie nu ook echt een beetje 'mijn ouders' geworden. Ik wil 
jullie bedanken voor jullie onvoorwaardelijke steun. Jullie staan werkelijk altijd voor ons klaar! Ik mag me gelukkig prijzen met zulke schoonouders, waar ik dag en nacht bij terecht kan!

Laurens, mijn lieve 'kleine' broertje. Hoewel je inmiddels veel groter bent dan ik zal je toch altijd mijn kleine broertje blijven. Wat hebben we toch veel mooie dingen meegemaakt samen! Jij hebt je inmiddels ontwikkeld tot een echte bussinessman, maar met 'hart' voor de zaak, en woont nu aan de andere kant van Nederland (als je tenminste niet op een van je wereldreizen bent;-)). Dat neemt niet weg dat ik je heel graag zie en super trots ben op wat jij allemaal hebt bereikt! Dank je wel dat je er altijd voor me bent, ook al zit je aan de andere kant van de wereld!

Ralph, sinds een jaar nu mijn echtgenoot en mijn rots in de branding. Dank je wel voor de onvoorwaardelijke steun die je me hebt gegeven in de afgelopen jaren. Zonder jou zou het nooit gelukt zijn mijn proefschrift af te maken. Afgezien van de tijd die je me hebt gegund, heb je me ook nog geweldig geholpen om mijn artikelen te stroomlijnen in goed leesbaar Engels. Ik kan altijd op je bouwen en als ik het soms even niet meer zag zitten had je wel weer een relativerend woord waarna ik me weer een stuk beter voelde. Dank je wel voor alles en ik hou ontzettend veel van je!

Lieve Silke, mijn prachtige dochter. Veel mensen vinden het knap dat ik ondanks dat ik jou heb gekregen, mijn proefschrift toch heb afgemaakt. Ik denk dat het juist andersom is (en dat ondanks dus dankzij moet zijn)! Het begon al bij het feit dat jij het wel lekker vond in mijn buik en besloot tot ver na de uitgerekende datum te blijven zitten. Hierdoor heb ik lekker lang kunnen werken aan mijn proefschrift tijdens mijn zwangerschapsverlof. Vanaf het moment dat je er was tot de dag van vandaag ben je echt een 'model' kind. Je bent bijna altijd vrolijk, je slaapt als een roos en je lust alles wat we je te eten geven. Dit heeft er voor gezorgd dat ik op mijn dagen met jou rustig de tijd had om dingen af te maken als jij bijvoorbeeld lekker lag te slapen. Jij ontneemt me geen energie maar geeft me juist energie. Ik ben apetrots op een dochter zoals jij!

Tot slot wil ik me graag richten tot mijn ouders. Lieve papa en mama, van alle personen ben ik jullie wel het allermeeste dank verschuldigd. Het lijkt misschien soms alsof ik het allemaal maar als 'normaal' beschouw wat jullie allemaal voor mij gedaan hebben en nog steeds doen, maar dat doe ik zeker niet. Jullie hebben mij de mogelijkheid en vrijheid gegeven om mij te ontwikkelen tot de persoon die ik nu ben. Ik heb gedurende mijn studie en daarna toen ik al aan het werk was en onderzoek deed, altijd op jullie positieve en stimulerende steun kunnen rekenen. Ik zal jullie hier altijd dankbaar voor zijn en ik hoop dat ik voor mijn kinderen in de toekomst hetzelfde kan betekenen als jullie voor mij. 
Curriculum vitae 


\section{CURRICULUM VITAE}

Mariëlle Coolsen was born on the 24th of January 1984 in Eindhoven, The Netherlands. After graduating from Rythovius College secondary school in 2002 (cum laude) she started studying medicine at Maastricht University graduating cum laude in 2008. During her study she worked as a student researcher with dr. Ronald M. van Dam. Here she laid the foundation for her later PhD research on Enhanced Recovery After Surgery (ERAS). In 2008 she was awarded a Kootstra fellowship for fundamental research in orthopedic surgery. It was in the orthopedic field where she started her surgical residency in July 2009. However, soon after she started her residence Mariëlle decided to switch to general surgery and worked for the remainder of her first two years under dr. R.J. Welten in Heerlen. In July 2011 Mariëlle continued her surgical residency under prof. L.P.S. Stassen in Maastricht. Recently, she returned to Heerlen for the last two years of her residency, now under the guidance of dr. M.N. Sosef, for the differentiation of surgical oncology. She plans to finish her residency in January 2016. In 2011 Mariëlle also started a PhD with prof. C.H.C. Dejong. This PhD continued where Mariëlle left off in her days as a student researcher and involved the application of ERAS protocols to liver and pancreatic surgery.

In her spare time Mariëlle is an active board member of the SEOHS committee and its national counterpart SWOAHS. Music and Latin dancing remain her most important hobbies. She is happily married, lives in Maastricht and is mother of a daughter. 
